\title{
A Measurement of Neutrino Charged Current Interactions and a Search for Muon Neutrino Disappearance with the Fermilab Booster Neutrino Beam
}

\author{
Yasuhiro Nakajima
}

\author{
A dissertation \\ submitted in partial fulfillment of the requirements \\ for the degree of Doctor of Science \\ in the Graduate School of Science, \\ Kyoto University \\ January, 2011
}





\begin{abstract}
In this thesis, we report on a measurement of muon neutrino inclusive charged current interactions on carbon in the few $\mathrm{GeV}$ region, using the Fermilab Booster Neutrino Beam. The all neutrino mode data collected in the SciBooNE experiment is used for this analysis.

We collected high-statistics CC interaction sample at SciBooNE, and extracted energy dependent inclusive charged current interaction rates and cross sections for a wide energy range from $0.25 \mathrm{GeV}$ to $\sim 3 \mathrm{GeV}$. We measure the interaction rates with $6-15 \%$ precision, and the cross sections with 10-30\% precision. We also made an energy integrated measurements, with the precisions of $3 \%$ for the rate, and $8 \%$ for the cross section measurements. This is the first measurement of the $\mathrm{CC}$ inclusive cross section on carbon around $1 \mathrm{GeV}$.

This inclusive interaction measurement is nearly free from effects of hadron re-interactions in the nucleus. Hence, it is complementary to other exclusive cross section measurements, and essential to understand the neutrino interaction cross sections in the few $\mathrm{GeV}$ region, which is relevant to ongoing and future neutrino oscillation experiments. This analysis also provides the normalization for SciBooNE's previous cross section ratio measurements for charged current coherent pion production and neutral current neutral pion production. Then, a precise comparison between our previous measurements and the model predictions becomes possible.

The result of the interaction rate measurement is used to constrain the product of the neutrino flux and the cross section at the other experiment on the Fermilab Booster Neutrino Beam: MiniBooNE. We conducted a search for short-baseline muon neutrino disappearance using data both from SciBooNE and MiniBooNE, to test a possible neutrino oscillation with sterile neutrinos which is suggested by the LSND experiment. With this constraint by SciBooNE, we significantly reduced the flux and the cross section uncertainties at MiniBooNE, and achieved the world best sensitivity for the $\nu_{\mu}$ disappearance at $0.5<\Delta m^{2}<30\left(\mathrm{eV}^{2}\right)$. We found no significant oscillation signal, and set one of the world strongest limits for the sterile neutrino models.
\end{abstract}




\section{Acknowledgements}

This thesis has never been completed without tremendous supports from many people. Here, I would like to acknowledge each of them.

First of all, I would like to express my deepest appreciation to my adviser, and a co-spokesperson for SciBooNE as well, Tsuyoshi Nakaya, who gave me such a wonderful opportunity to work on this research. It all begun five years ago, when I switched my research topic to neutrino physics and joined to the SciBooNE experiment. Your continuous advices, supports and encouragements always led me to the right direction toward the completion of this analysis. I would particularly like to thank Masashi Yokoyama, who lived "on-site" with us, and led the SciBooNE detector construction/commissioning and the initial data analysis. Your advices and discussions in Japanese significantly helped me to work in the MRD group, in which I was the only Japanese. Without you, the MRD detector had never be constructed in a timely manner. I am so grateful to Morgan Wascko, another co-spokesperson for SciBooNE, for leading the collaboration and giving me various advices. Especially, thank you for organizing so many social events and connecting the collaborators strongly. I really like this collaboration because of you.

I would like to express my sincere appreciation to Katsuki Hiraide and Yoshinori Kurimoto, who shared most of the life at Fermilab for more than two years. We were so different, but I really enjoyed discussing physics and sharing lots of activities with you. I am proud that I worked with you.

For the MRD construction, I worked closely with Robert Napora and Joseph Walding. Thank you Rob for leading the MRD construction. At the beginning, I had a really hard time listening and speaking English. Even though, you welcomed me as a member of MRD group and gave me many opportunities to work on various aspects of the MRD construction. Thank you Joe for sharing most of the MRD works, not only on the construction, but also on the commissioning and the data analysis. My sincere gratitude goes to Richard Tesarek, who led the construction of the SciBooNE detector as the project manager. I really enjoyed the construction of MRD because of so many people I worked with, especially Paul Nienaber and Len Bugel. Thank you for organizing and sharing the construction work from the beginning. I would like to thank the technicians and engineers at Fermilab who helped building the detectors, especially to Karen Kephart and John Voirin for various supports for building the MRD.

For the data analysis, I would like to express my thanks particularly to our analysis coordinators, Hidekazu Tanaka and Michel Sorel. Thank you for and organizing all the analysis works and lots of valuable advices. I am grateful to my co-worker for the SciBooNE-MiniBooNE joint analysis, Kendall Mahn, for all the help especially on the works with MiniBooNE group. This analysis had never been possible without you. Also, thank you Kendall for helping my life in the US and organising lots of "off-site" activities.

I would like to express my gratitude to Mike Shaevitz, for leading us towards this SciBooNEMiniBooNE joint analysis. I enjoyed discussions with you. I would like to thank Yoshinari Hayato and Sam Zeller, for advices especially on the neutrino interaction simulations. I am thankful to Lucio Ludovici and Rex Taylor, for various advices on my neutrino interaction measurements. I would like to express my deep appreciation to Chris Polly, Richard Van de Water, Steve Brice and Bill Luis, for helping my analysis from MiniBooNE side. Your advices not only improved my 
analysis a lot, but also added a really strong motivation to me.

I really enjoyed this work and the life at Fermilab because of so many wonderful fellow coworkers. Especially, I would like to express my thanks to Teppei Katori, who taught me not only physics but also how to enjoy a life in Chicago. I was impressed with your enthusiasm to both physics and your private life. I am grateful to Gaku Mitsuka, for helping the commissioning of the MRD. Although it was just for a few months, I was really happy to share the first data from the MRD and excitement of getting the detector work with you. I would like to express many thanks to all of those who shared the physics analysis and the life at the Fermilab, especially to: Hideyuki Takei, Camillo Mariani, Claudio Giganti, Jose Alcaraz, Joan Catala, Gary Cheng, and Aaron Hanson. Thank you. I am thankful to SciBooNE members from Kyoto University and Tokyo-tech who helped construction and/or decommissioning of the SciBooNE detectors: K. Matsuoka, H. Kubo, M. Otani, D. Orme, Y. Kobayashi, S. Masuike and S. Mizugashira. I must thank S. Kunori and K. Kunori, for supporting the life at Fermilab and inviting me to so many parties, which gave me precious opportunities to know Japanese people working at Fermilab.

During the first two years in the graduate school, I was involved in Kaon decay experiments, which gave me precious knowledge and experiences for the experimental particle physics. If there were not that two years, my thesis work had never been possible. Especially, I am deeply grateful to my former adviser, Noboru Sasao. I learned from you not only knowledge and techniques, but also the attitude to carry on physics experiments. I would like to express my sincere thanks to Tadashi Nomura and Hajime Nanjo for lots of advises and discussions.

I wish to extend my thanks to the current and former members of the high energy physics group at Kyoto University: K. Nishikawa, A. Ichikawa, M. Suehiro, T. Fujiwara, H. Yokoyama, I. Kato, Y. Honda, H. Maesaka, K. Mizouchi, M. Hasegawa, T. Sumida, S. Yamamoto, A. Minamino, K. Nitta, H. Morii, J. Kubota, N. Taniguchi, K. Takezawa, T. Shirai, K. Ezawa, Y. Kurosawa, T. Nobuhara, M. Taguchi, H. Kawamuko, S. Gomi, K. Shiomi, T. Usuki, N. Kawasaki, N. Nagai, T. Masuda, K. Ieki, D. Naito, Y. Maeda, A. Murakami, T. Kikawa, K. Suzuki, G. Takahashi, S. Takahashi, T. Hiraki and T. Yamauchi. Especially, I would like to thank Atsuko Ichikawa, who shared the office with me at Kyoto and always encouraged me to complete my thesis. I am thankful to the secretaries at Kyoto University, especially to A. Nakao, M. Mori, N. Hirano, M. Hiraoka, K. Nakagawa, Y. Nishikubo and M. Tanizawa, for taking care of every businesses.

I was also supported by so many friends that I cannot list each name here. Thank you for sharing lots of entertainments with me, which always gave me energy to move forward. I wish our friendship will last for the rest of my life.

Finally, I would like to express my special thanks to my parents, for supporting and understanding my work and life to this day.

Thank you. 


\section{Contents}

1 Introduction 1

1.1 Neutrinos in the standard model . . . . . . . . . . . . . . . . 1

1.2 Neutrino mass and mixing . . . . . . . . . . . . . . . . . 1

1.2.1 Mixing with Dirac mass term . . . . . . . . . . . . . . 2

1.2 .2 Mixing with Majorana mass term . . . . . . . . . . . . . 2

1.3 Neutrino oscillation . . . . . . . . . . . . . . . . . . . . 3

1.3.1 Oscillation with three active flavors . . . . . . . . . . . 4

1.3.2 Neutrino oscillation with sterile neutrinos . . . . . . . . . . . . 6

$1.4 \nu_{\mu}$ disappearance search with the Fermilab Booster Neutrino Beam . . . . . . . . . . 8

1.5 Measurement of inclusive charged current interactions . . . . . . . . . . . . . . 10

1.5.1 Importance of inclusive charged current interaction measurements . . . . . . 10

1.5.2 Benefits to the measurements of exclusive channels . . . . . . . . . . . . . 11

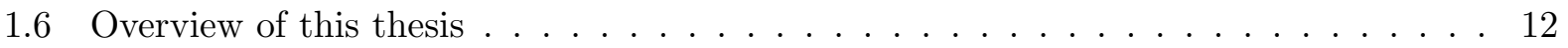

2 Experimental technique 14

2.1 Overview of the experimental setup . . . . . . . . . . . . . . . . 14

2.1 .1 SciBooNE experiment . . . . . . . . . . . . . . . . . 14

2.1 .2 MiniBooNE experiment . . . . . . . . . . . . . . . 15

2.2 Principle of the measurements . . . . . . . . . . . . . . . . . . 15

2.2 .1 Neutrino oscillation analysis . . . . . . . . . . . . . . 15

2.2.2 Measurement of neutrino interaction cross section $\ldots \ldots \ldots \ldots \ldots$

2.3 Analysis strategy . . . . . . . . . . . . . . . . . . . 17

3 Experimental apparatus and the data sets $\mathbf{1 8}$

3.1 Experimental apparatus . . . . . . . . . . . . . . . . . . 18

3.1 .1 Booster Neutrino Beam . . . . . . . . . . . . . . . . . . 18 18

3.1.1.1 Target and magnetic focusing horn . . . . . . . . . . . . . 18

3.1.1.2 Decay region and absorber . . . . . . . . . . . 19 19

3.1.2 SciBooNE detector . . . . . . . . . . . . . . . . . . . . . . . 19

3.1.2.1 Scintillator bar tracker (SciBar) . . . . . . . . . . . . 19

3.1.2.2 Electromagnetic Calorimeter (EC) . . . . . . . . . . . . . 20

3.1.2.3 Muon Range Detector (MRD) . . . . . . . . . . . . . . . . . 23

3.1.2.4 Trigger and data acquisition (DAQ) system . . . . . . . . . . . 25

3.1 .3 MiniBoonE detector . . . . . . . . . . . . . . . . . . . 25

3.2 Data sets . . . . . . . . . . . . . . . . . . . 27

4 Monte carlo simulation $\quad 29$

4.1 Neutrino flux simulation . . . . . . . . . . . . . . . . . . . . 29

4.1.1 Simulation of meson productions . . . . . . . . . . . . . 29

4.1 .2 Simulation of meson decays . . . . . . . . . . . . . . . . 30

4.1 .3 Neutrino beam flux prediction . . . . . . . . . . . . . . 31 
4.1.4 Systematic uncertainty of the flux prediction . . . . . . . . . . 31

4.2 Neutrino interaction simulation . . . . . . . . . . . . . . . 34

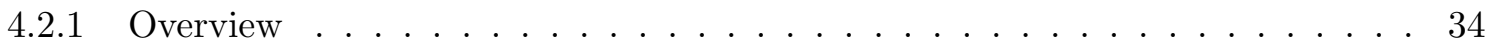

4.2 .2 Neutrino interaction model . . . . . . . . . . . . . . . . 34

4.2.2.1 Quasi-elastic scattering . . . . . . . . . . . . . . . 34

4.2.2.2 Meson production via baryon resonances . . . . . . . . . . 355

4.2.2.3 Coherent pion production . . . . . . . . . . . . . . . 35

4.2.2.4 Deep inelastic scattering . . . . . . . . . . . . . 36

4.2.2.5 Intra-nuclear interactions . . . . . . . . . . . . . . . 36

4.2.3 The expected number of neutrino events . . . . . . . . . . 36

4.3 SciBooNE detector simulation . . . . . . . . . . . . . . . . . . 37

4.3.1 Simulation of particle propagation in detector $\ldots \ldots \ldots \ldots$

4.3.2 Simulation of detector response . . . . . . . . . . . . . . 39

4.3.3 Neutrino interactions in the surrounding material . . . . . . . . . . . . . 39

5 Event reconstruction in SciBooNE detector 40

5.1 Track reconstruction . . . . . . . . . . . . . . . . 40

5.1 .1 Track finding . . . . . . . . . . . . . . . . 40

5.1 .2 Track classification . . . . . . . . . . . . . . . . . . . 41

5.1 .3 Particle identification . . . . . . . . . . . . . . . 41

5.1 .4 Muon kinematics reconstruction . . . . . . . . . . . . . . 42

5.1.4.1 Muon angle reconstruction . . . . . . . . . . . . . . 42 42

5.1.4.2 Muon momentum reconstruction . . . . . . . . . . . . . . . 43

5.2 Event selection and classification . . . . . . . . . . . . . . . . 43

5.2 .1 Event selection . . . . . . . . . . . . . . . . 43

5.2 .1 .1 Validity of the $p_{\mu}$ cut $\ldots \ldots \ldots \ldots 4$

5.2 .2 Event classification . . . . . . . . . . . . . . . . . . . 444

5.2 .2 .1 SciBar-stopped sample . . . . . . . . . . . . . . 444

$5.2 .2 .2 \quad$ MRD-stopped sample . . . . . . . . . . . . . . 46

5.2 .2 .3 MRD-penetrated sample . . . . . . . . . . . . . 48

5.2 .3 Efficiency summary . . . . . . . . . . . . . . . . . . 48

5.3 Data comparison to the MC prediction . . . . . . . . . . . . . 50

6 Measurement of CC interactions at SciBooNE 52

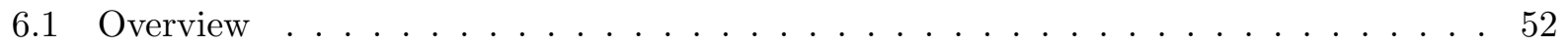

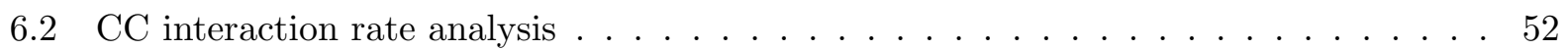

6.2 .1 Method . . . . . . . . . . . . . . . . . . . . 52

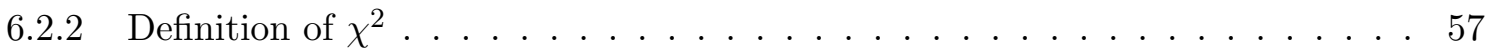

6.2 .3 Error analysis . . . . . . . . . . . . . . . . 5 57

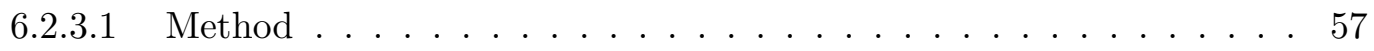

6.2.3.2 The size of systematic uncertainties . . . . . . . . . . . 59

6.2 .4 Spectrum fit result . . . . . . . . . . . . . . . . . . 61

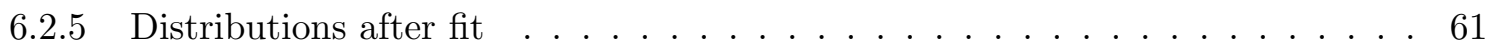

6.3 Results and discussions . . . . . . . . . . . . . . . . 66

6.3.1 Energy dependent $\mathrm{CC}$ interaction rate and cross section . . . . . . . . 66

6.3.1.1 CC interaction rate extraction . . . . . . . . . . . . 66

6.3.1.2 CC inclusive cross section . . . . . . . . . . . . . 66 66

6.3.2 Flux integrated total CC interaction rate and cross section . . . . . . . 68

6.3.2.1 Measurement of $\mathrm{CC}$ interaction rate . . . . . . . . . . . 68 68

6.3.2.2 CC inclusive cross section . . . . . . . . . . . . . 69 69

6.3.3 Discussion of the CC rate and cross section measurement results . . . . . . 69 
6.3.3.1 Consistency between NEUT and NUANCE . . . . . . . . . . . . 699

6.3.3.2 Comparison with other CC measurements . . . . . . . . . . . 70

6.4 Applications to other measurements . . . . . . . . . . . . . . . . 70

6.4.1 Absolute normalization for previous SciBooNE measurements . . . . . . . . 72

6.4.2 SciBooNE-MiniBooNE joint $\nu_{\mu}$ disappearance analysis . . . . . . . . . 73

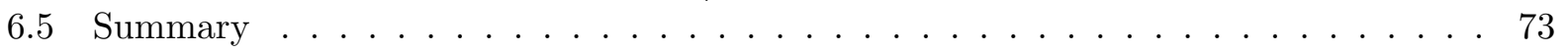

7 Search for muon neutrino disappearance $\mathbf{7 4}$

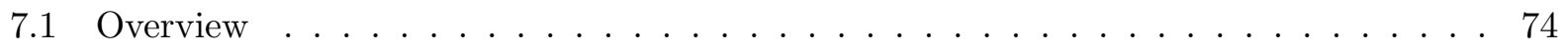

7.2 MiniBooNE event reconstruction and prediction . . . . . . . . . . . . . 74

7.3 MiniBoonE data sample . . . . . . . . . . . . . . . . . . . . . . 74

7.4 Prediction of MiniBooNE events with the SciBooNE constraints . . . . . . . . 75

7.4 .1 Central value prediction . . . . . . . . . . . . . 75

7.4 .2 Systematic uncertainties . . . . . . . . . . . . . . . 76

7.4.2.1 Errors of the SciBooNE CC interaction measurement . . . . . . . 777

7.4.2.2 Errors of the ratio between SciBooNE and MiniBooNE distributions 77

7.4 .2 .3 Stability errors . . . . . . . . . . . . . . . 78

7.4 .3 Final prediction . . . . . . . . . . . . . . . 81

7.5 Test of neutrino oscillation hypothesis $\ldots \ldots \ldots \ldots \ldots$

7.5.1 Prediction of oscillation signal . . . . . . . . . . . . . 83

7.5.1.1 Overview . . . . . . . . . . . . . . . 83

7.5.1.2 Oscillation effect at SciBooNE . . . . . . . . . . . . 84

7.5.1.3 Oscillation prediction at MiniBooNE . . . . . . . . . . . 87

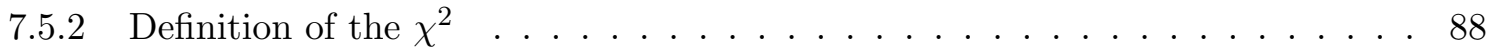

7.5.2.1 Fit for the new data . . . . . . . . . . . . 88

7.5.2.2 New and old data combined fit . . . . . . . . . . . . 88

7.5.3 Confidence level determination . . . . . . . . . . . . . . . . . . 899

7.6 Sensitivity . . . . . . . . . . . . . . . . . . . . . . . . . . . . . . 89

7.7 Result and discussions . . . . . . . . . . . . . . . . . . . . . . . . . . . . . . .

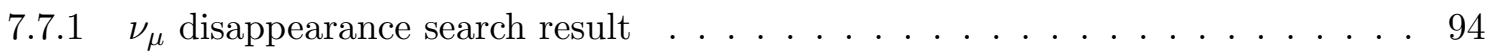

7.7 .2 Possible improvements . . . . . . . . . . . . . . . . . . . 94

7.7.3 Muon anti-neutrino disappearance . . . . . . . . . . . . . . . 94

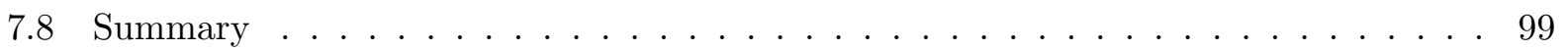

8 Conclusions 101

A Study of the spectrum fit methods $\quad 103$

A.1 Choice of samples and binning . . . . . . . . . . . . . . . . 103

A.1.1 Energy resolution . . . . . . . . . . . . . . . . . . . 103

A.1.2 Acceptance comparison to MiniBooNE . . . . . . . . . . . . . . . 103 . . . . . . . . . . .

A.1.3 Sample and binning . . . . . . . . . . . . . . . . 103

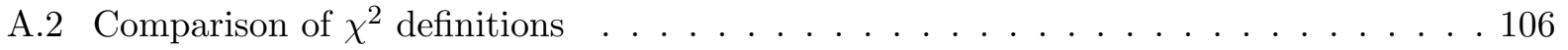

A.2.1 Likelihood ratio . . . . . . . . . . . . . . . . . 106

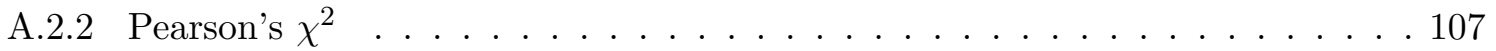

A.2.3 Comparison between the two methods . . . . . . . . . . . . . . . 107

B CC single $\pi$ production $Q^{2}$ shape measurement 109

B.1 Sample to fit . . . . . . . . . . . . . . . . . . . . . . 109

B.2 Fit method and result . . . . . . . . . . . . . . . . . 110

B.3 Comparison to the correction factor obtained from MiniBooNE data . . . . . . . 112 
C Simulation and event reconstruction in MiniBooNE 113

C.1 MiniBooNE detector simulation . . . . . . . . . . . . . . . . . . . . . . 113

C.1.1 MiniBooNE detector response error . . . . . . . . . . . . . . . . 113

C.2 Selection of beam-induced events . . . . . . . . . . . . . . . . . 114

C.3 Track reconstruction . . . . . . . . . . . . . . . . . . . . . . . 115

C.4 Event selection in MiniBooNE . . . . . . . . . . . . . . . . . . . . . . . .116

C.5 Neutrino energy reconstruction . . . . . . . . . . . . . . . . . 119 



\section{Chapter 1}

\section{Introduction}

\subsection{Neutrinos in the standard model}

Neutrinos are elementary particles with spin $1 / 2$ and zero electrical charge, and don't make the strong interaction. This particle is originally postulated by W. Pauli to explain the continuum electron energy spectrum of the $\beta$ decay, and now it is known that there are three types of neutrinos, $\nu_{e}, \nu_{\mu}$ and $\nu_{\tau}$.

The direct mass measurements are performed by measuring kinematics of particle decays. However, as shown in the Table 1.1, no finite absolute mass has ever measured. The number of neutrino generations is measured in the LEP experiments using invisible decay of $Z$ boson, to be $N_{\nu}=2.984 \pm 0.008$ [1]. Hence the possibility of fourth weakly interacting light neutrino $\left(m_{\nu}<M_{Z} / 2\right)$ is excluded.

Table 1.1: Present limits of neutrino masses

\begin{tabular}{ccc}
\hline \hline Neutrino & Mass limit & Experiment \\
\hline$\nu_{e}$ & $2.3 \mathrm{eV} / c^{2}(95 \% \mathrm{CL})$ & Tritium $\beta$ decay [2] \\
$\nu_{\mu}$ & $0.17 \mathrm{MeV} / c^{2}(90 \% \mathrm{CL})$ & Pion decay [3] \\
$\nu_{\tau}$ & $18.2 \mathrm{MeV} / c^{2}(95 \% \mathrm{CL})$ & Tau decay [4] \\
\hline \hline
\end{tabular}

From these facts, in the current standard model (SM) of particle physics, neutrinos are realized as mass-less particles which only have chirally left-handed components $\left(\nu_{L}\right)$, and they form weak isospin doublets with charged leptons as,

$$
\left(\begin{array}{c}
\nu_{L}^{e} \\
e_{L}
\end{array}\right),\left(\begin{array}{c}
\nu_{L}^{\mu} \\
\mu_{L}
\end{array}\right) \text { and }\left(\begin{array}{c}
\nu_{L}^{\tau} \\
\tau_{L}
\end{array}\right) .
$$

The right-handed components of neutrinos $\left(\nu_{R}\right)$ are not included in the framework of the SM.

However, as discussed in the following sections, the observations of the neutrino oscillations show that the neutrinos have finite masses. This opened the door to further explore the nature of neutrinos beyond the SM.

\section{$1.2 \quad$ Neutrino mass and mixing}

Although the SM contains only the left-handed component of neutrinos, one can also define the right-handed component, $\nu_{R}$. This $\nu_{R}$ plays a crucial role in realization of the finite neutrino mass and prediction of the so-called "sterile neutrino". In this section, we briefly describe the theoretical framework of neutrino mass and mixing, following discussions in Refs. [5, 6]. 


\subsubsection{Mixing with Dirac mass term}

To realize massive neutrinos, a Dirac mass term may be constructed by introducing right-handed neutrino fields $\nu_{R}^{\alpha}(\alpha=e, \mu, \tau)$ in addition to the left-handed field $\nu_{L}^{\alpha}$ :

$$
\mathcal{L}_{\text {mass }}^{D}=-\bar{\nu}_{R}^{\alpha} m_{\alpha \beta} \nu_{L}^{\beta}+\text { h.c. }
$$

Here, $\nu^{\alpha}$ are the eigenstates of the weak interaction specified by the Lagrangian of the weak charged current interaction,

$$
\mathcal{L}_{\text {int }}=\frac{g}{\sqrt{2}} W_{\mu}^{+}\left(\bar{\nu}_{L}^{e} \gamma^{\mu} e_{L}+\bar{\nu}_{L}^{\mu} \gamma^{\mu} \mu_{L}+\bar{\nu}_{L}^{\tau} \gamma^{\mu} \tau_{L}\right)+\text { h.c. }
$$

where $W$ is the charged weak boson, and $g$ is the semi-weak coupling constant of the SM, $g=$ $e / \sin \theta_{W}$, with $e$ being the electric charge of the positron and $\theta_{W}$ the Weinberg angle.

In general, the weak eigenstates of neutrinos can be expressed as superpositions of the mass eigenstates $\nu^{i}(i=1,2,3)$;

$$
\begin{aligned}
\nu_{L}^{\alpha} & =U_{\alpha i} \nu_{L}^{i}, \\
\nu_{R}^{\alpha} & =V_{\alpha i} \nu_{R}^{i},
\end{aligned}
$$

which diagonalize the mass term as,

$$
\begin{aligned}
\mathcal{L}_{\text {mass }}^{D} & =-\bar{\nu}_{R}^{i} V_{i \alpha}^{\dagger} m_{\alpha \beta} U_{\beta j} \nu_{L}^{j}+\text { h.c. } \\
& =-\bar{\nu}_{R}^{i} m_{i} \nu_{L}^{i}+\text { h.c. }
\end{aligned}
$$

Here,

$$
V^{\dagger} m U=\left(\begin{array}{ccc}
m_{1} & & 0 \\
& m_{2} & \\
0 & & m_{3}
\end{array}\right),
$$

where $m_{i}(i=1,2,3)$ are mass values corresponds to the mass eigenstates $\nu^{i}$. Using this basis of the mass eigenstates, the interaction Lagrangian Eq. (1.3) can be rewrote as

$$
\mathcal{L}_{\text {int }}=\frac{g}{\sqrt{2}} W_{\mu}^{+}\left(\bar{\nu}^{1}, \bar{\nu}^{2}, \bar{\nu}^{3}\right)_{L} U^{\dagger} \gamma_{\mu}\left(\begin{array}{c}
e \\
\mu \\
\tau
\end{array}\right)_{L}+\text { h.c. },
$$

where $U$ is referred to as Maki-Nakagawa-Sakata (MNS) matrix [7].

\subsubsection{Mixing with Majorana mass term}

In addition to the Dirac mass term, one can also define a Majorana mass term, which is constructed by $\nu_{L}$ or $\nu_{R}$ alone, such as

$$
\mathcal{L}_{\text {mass }}^{M}=-\frac{1}{2} \overline{\nu^{c}}{ }_{\alpha R} m_{\alpha \beta}^{R} \nu_{\beta R}+\text { h.c. }
$$

where the superscript $c$ denotes charge conjugation, defined as $\nu^{c}=C \bar{\nu}^{T}$, where $C$ is the charge conjugation operator. The effect of this operation is to convert the fundamental neutrino fields into their own antiparticles. By adding a Majorana mass term, the neutrino mass Lagrangian becomes

$$
\mathcal{L}_{\text {mass }}=\mathcal{L}_{\text {mass }}^{D}+\mathcal{L}_{\text {mass }}^{M}=-\bar{\nu}_{R}^{\alpha} m_{\alpha \beta} \nu_{L}^{\beta}-\frac{1}{2} \overline{\nu^{c}}{ }_{\alpha R} m_{\alpha \beta}^{R} \nu_{\beta R}+\text { h.c.. }
$$

This mass term can be rewrote as

$$
\mathcal{L}_{\text {mass }}=\left(\bar{\nu}_{\alpha L}^{c}, \bar{\nu}_{\alpha R}\right)\left(\begin{array}{cc}
0 & m^{T} \\
m & m^{R \dagger}
\end{array}\right)\left(\begin{array}{c}
\nu_{\alpha L} \\
\nu_{\alpha R}^{c}
\end{array}\right)+\text { h.c. }
$$


Then, we can also define the mass eigenstates $\nu_{i L}$ and $\nu_{i R}^{c}(i=1,2,3)$ as

$$
\left(\begin{array}{c}
\nu_{\alpha L} \\
\nu_{\alpha R}^{c}
\end{array}\right)=U\left(\begin{array}{c}
\nu_{i L} \\
\nu_{i R}^{c}
\end{array}\right)
$$

Here, $U$ is a $6 \times 6$ unitary matrix which satisfies

$$
U^{\dagger}\left(\begin{array}{cc}
0 & m^{T} \\
m & m^{R \dagger}
\end{array}\right) U=M_{\text {diag }}
$$

where $M_{\text {diag }}$ is a diagonal matrix which contains mass eigenvalues. Hence, $\nu_{L}$ and $\nu_{R}^{c}$ can also mix if neutrino has the Majorana mass term constructed by right-handed field, in addition to the Dirac mass term.

To estimate the size of mixing, we consider a case that there is only one generation for both $\nu_{L}$ and $\nu_{R}^{c}$. If we assume the Majorana mass $m_{R}$ to be much larger than the Dirac mass $m, U$ is approximately written as

$$
U \simeq\left(\begin{array}{cc}
1 & \rho \\
-\rho & 1
\end{array}\right)\left(\begin{array}{ll}
i & 0 \\
0 & 1
\end{array}\right)
$$

where $\rho=m / m_{R} \ll 1$. Using this $U$ in Eq. 1.15), the diagonalized mass matrix $\left(M_{\text {diag }}\right)$ become

$$
M_{\text {diag }} \simeq\left(\begin{array}{cc}
m^{2} / m^{R} & 0 \\
0 & m^{R}
\end{array}\right)
$$

Hence, if $m^{R} \gg m$, the mixing between $\nu_{L}$ and $\nu_{R}^{c}$ become negligible. Also if we set the Dirac mass $m$ to be the order of a typical quark or charged lepton mass, the mass of $\nu_{1}^{L}$,

$$
m_{1} \simeq m^{2} / m^{R}
$$

can be very small. Thus, if we identify $\nu_{1}^{L}$ as one of the light neutrinos, we have an elegant explanation of why their masses are so small compared to other elementary particles. This explanation, in which physical neutrino masses are small because the right-handed Majorana mass $m^{R}$ is large, is known as the see-saw mechanism, and Eq. (1.17) is referred to as the see-saw relation [8 11].

However, if $m^{R}$ is small enough, the mixing between $\nu_{\alpha L}$ and $\nu_{\beta R}^{c}$, as well as $\nu_{\alpha L}$ and $\nu_{\beta L}$, become possible [6]. The $\nu_{R}^{c}$ is gauge singlet and don't make interactions with detectors, and hence is called "sterile neutrino". Since the sterile neutrinos don't make weak interactions, this can be "observed" only through interferences of mass eigenstates.

\subsection{Neutrino oscillation}

If there is a mixing between the weak and mass eigenstates, neutrinos can change their flavor (or, weak eigenstates) as a function of time due to interference of the different mass eigenstates, which called "neutrino oscillation". In this section, we describe the phenomenology of the neutrino oscillation and summary of the previous oscillation measurements.

To discuss the phenomenology of the neutrino oscillation, we generally define three active flavor eigenstates as $\left(\nu_{e}, \nu_{\mu}, \nu_{\tau}\right)=\left(\nu_{e L}, \nu_{\mu L}, \nu_{\tau L}\right)$ and sterile flavors as $\left(\nu_{s_{1}}, \nu_{s_{2}}, \nu_{s_{3}}\right)=\left(\nu_{e R}^{c}, \nu_{\mu R}^{c}, \nu_{\tau R}^{c}\right)$. The mass eigenstates are also defined as $\left(\nu_{1}, \nu_{2}, \cdots, \nu_{6}\right)=\left(\nu_{1 L}, \nu_{2 L}, \nu_{3 L}, \nu_{1 R}^{c}, \nu_{2 R}^{c}, \nu_{3 R}^{c}\right)$.

Then, a flavor eigenstate of neutrino, $\left|\nu_{\alpha}\right\rangle\left(\alpha=e, \mu, \tau, s_{1}, s_{2}, s_{3}\right)$, can be expressed as a superposition of mass eigenstates, $\left|\nu_{i}\right\rangle(i=1,2,3, \cdots)$;

$$
\left|\nu_{\alpha}\right\rangle=\sum_{i} U_{\alpha i}\left|\nu_{i}\right\rangle
$$


where $U_{\alpha i}$ is an element of the MNS mixing matrix $U$. Generated as $\nu_{\alpha}$, the state of neutrino at time $t$ is expressed as

$$
|\nu(t)\rangle=\sum_{i} U_{\alpha i} e^{-i E_{i} t}\left|\nu_{i}\right\rangle
$$

where $E_{i}$ is the energy of $\nu_{i}$ in the laboratory frame. In practice, neutrino is extremely relativistic due to the tinniness of the mass, and thus we can make following approximations:

$$
\begin{array}{r}
t \sim L, \\
E_{i}=\sqrt{p_{i}^{2}+m_{i}^{2}} \sim p_{i}+\frac{m_{i}^{2}}{2 p_{i}},
\end{array}
$$

where $L$ is the distance traveled and $p_{i}$ is the momentum of $\nu_{i}$. Since $\nu_{\alpha}$ is produced with a definite momentum $p$, all of $\nu_{\alpha}$ 's mass eigenstates have a common momentum. Thus, the probability $P\left(\nu_{\alpha} \rightarrow \nu_{\beta}\right)$ that $\nu_{\beta}$ is observed after $\nu_{\alpha}$ travels the distance $L$ is given by

$$
\begin{aligned}
P\left(\nu_{\alpha} \rightarrow \nu_{\beta}\right)= & \left|\left\langle\nu_{\beta} \mid \nu(t)\right\rangle\right|^{2}=\left|\sum_{i} U_{\alpha i} U_{\beta i}^{*} e^{-i p L} e^{-i \frac{m_{i}^{2} L}{2 p}}\right|^{2} \\
= & \delta_{\alpha \beta}-4 \sum_{i>j} \operatorname{Re}\left(U_{\alpha i} U_{\beta i}^{*} U_{\alpha j}^{*} U_{\beta j}\right) \sin ^{2}\left[1.27 \Delta m_{i j}^{2} \frac{L}{E}\right] \\
& -2 \sum_{i>j} \operatorname{Im}\left(U_{\alpha i} U_{\beta i}^{*} U_{\alpha j}^{*} U_{\beta j}\right) \sin ^{2}\left[2.54 \Delta m_{i j}^{2} \frac{L}{E}\right]
\end{aligned}
$$

where $\Delta m_{i j}^{2} \equiv m_{i}^{2}-m_{j}^{2}$ is the mass squared difference between $\nu_{i}$ and $\nu_{j}$ in $\mathrm{eV}^{2}, L$ is in $\mathrm{km}$, and $E$ is in GeV. The sign of the last term in Eq. 1.22 is + instead of - in the case of the expression for anti-neutrino. Therefore, the neutrino oscillation occurs only when $\Delta m^{2} \neq 0$. As described in the following part, this phenomenon is observed and confirmed by several experiments. This is the evidence that the neutrinos have finite masses.

\subsubsection{Oscillation with three active flavors}

In the case of oscillation between three active neutrino flavors, the MNS matrix is expressed using four independent parameters: three mixing angles, $\theta_{12}, \theta_{23}, \theta_{13}$, and one complex phase $\delta$;

$$
\begin{aligned}
U & =\left(\begin{array}{ccc}
1 & 0 & 0 \\
0 & c_{23} & s_{23} \\
0 & -s_{23} & c_{23}
\end{array}\right)\left(\begin{array}{ccc}
c_{13} & 0 & s_{13} e^{-i \delta} \\
0 & 1 & 0 \\
-s_{13} e^{i \delta} & 0 & c_{13}
\end{array}\right)\left(\begin{array}{ccc}
c_{12} & s_{12} & 0 \\
-s_{12} & c_{12} & 0 \\
0 & 0 & 1
\end{array}\right) \\
& =\left(\begin{array}{ccc}
c_{12} s_{13} & s_{12} c_{13} & s_{13} e^{-i \delta} \\
-s_{12} c_{23}-c_{12} s_{13} s_{23} e^{i \delta} & c_{12} c_{23}-s_{12} s_{13} s_{23} e^{i \delta} & c_{13} s_{23} \\
s_{12} s_{23}-c_{12} s_{13} c_{23} e^{i \delta} & c_{12} s_{23}-s_{12} s_{13} c_{23} e^{i \delta} & c_{13} c_{23}
\end{array}\right),
\end{aligned}
$$

where $c_{i j}=\cos \theta_{i j}$ and $s_{i j}=\sin \theta_{i j}$. In case of $\delta \neq 0$, the MNS matrix includes the imaginary parts, which means the $\mathrm{CP}$ violation in the lepton sector. Hence, the $\delta$ is called the CP phase.

Because of the condition $\Delta m_{12}^{2}+\Delta m_{23}^{2}+\Delta m_{31}^{2}=0$, the number of independent parameters for neutrino oscillations is six in the case of three lepton generations: three mixing angles, $\left(\theta_{12}, \theta_{23}, \theta_{31}\right)$, one $\mathrm{CP}$ phase, $\delta$, and any two out of three mass squared difference, $\Delta m^{2}$ 's.

There are many neutrino oscillation measurements such as atmospheric neutrino observations, solar neutrino observations, reactor neutrino experiments and accelerator neutrino experiments. Figure 1.1 shows allowed or excluded regions from various experiments. In summary, there are two allowed regions observed and confirmed by several experiments: 
1. Atmospheric region: $\Delta m_{23}^{2} \sim 2.5 \times 10^{-3} \mathrm{eV}^{2}, \theta_{23} \sim 45$ degrees

The neutrino oscillation in the atmospheric region was discovered with the $\nu_{\mu} \rightarrow \nu_{x}$ oscillation by the Super-Kamiokande (SK) group [12. This is the first discovery of the neutrino oscillation. The result has been confirmed by two long baseline accelerator neutrino experiments (K2K [13] and MINOS [14]).

\section{Solar region: $\Delta m_{12}^{2} \sim 8 \times 10^{-5} \mathrm{eV}^{2}, \theta_{12} \sim 30$ degrees}

The neutrino oscillation in the solar region was discovered with $\nu_{e} \rightarrow \nu_{x}$ oscillation by solar neutrino experiments (Homestake [15], GALLEX [16], GNO [17], SAGE [18], SNO [19], SK [20] and Borexino [21]) and confirmed by a reactor neutrino experiment; KamLAND [22].

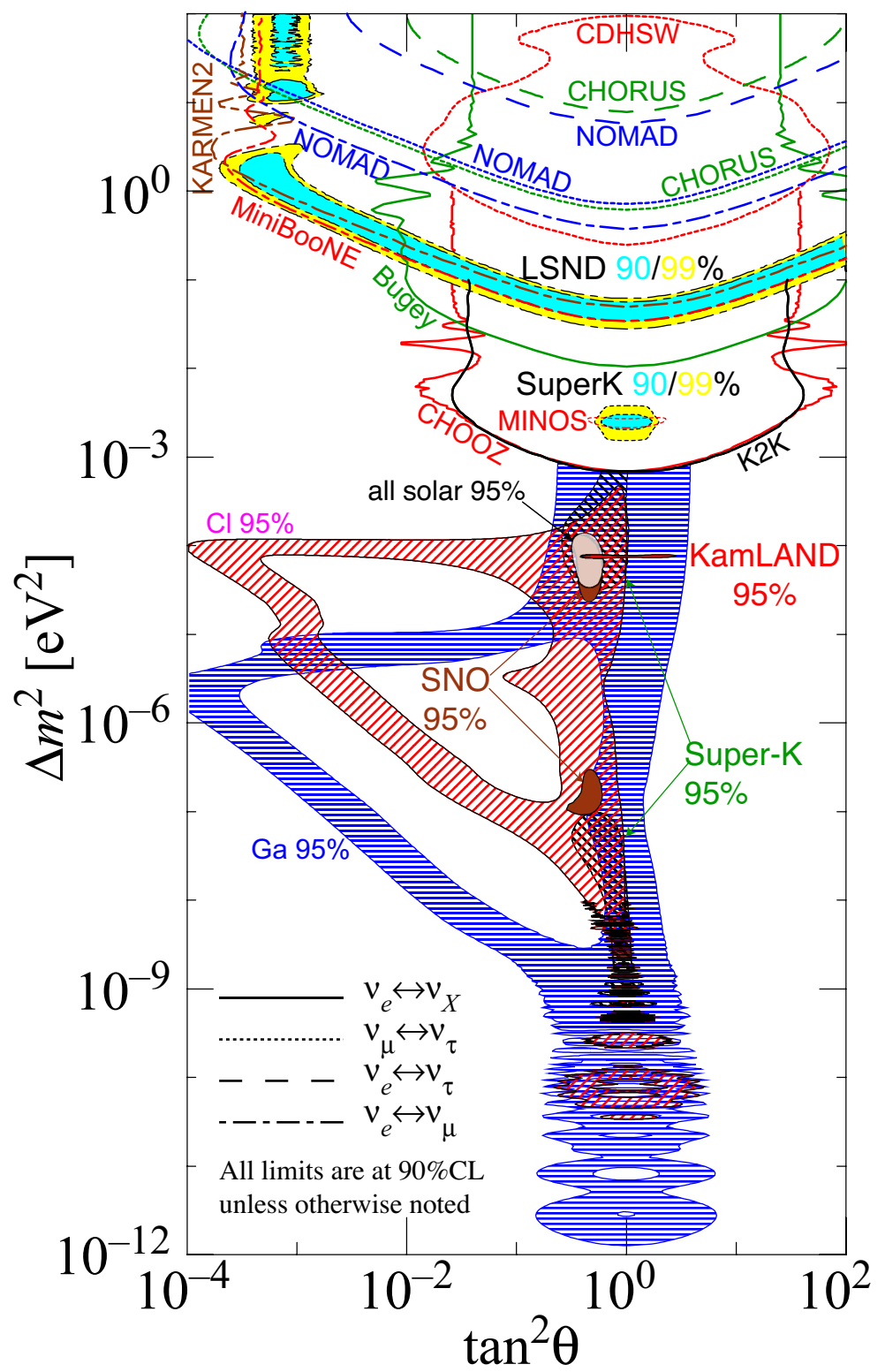

http://hitoshi.berkeley.edu/neutrino

Figure 1.1: Allowed or excluded regions in the $\tan ^{2} \theta-\Delta m^{2}$ plane by various experiments. There are two allowed regions around $\Delta m^{2} \sim 10^{-3}\left(\tan ^{2} \theta \sim 1\right)$ and $\Delta m^{2} \sim 10^{-5}\left(\tan ^{2} \theta \sim 1 / 3\right)$, which corresponds to the atmospheric and solar regions, respectively. In addition, there is one allowed region around $\Delta m^{2} \sim 1$, which is claimed by the LSND experiment.

In addition, there is one allowed region around $\Delta m^{2} \sim 1$, which is claimed by the LSND 
experiment [23. However, there is no room to accommodate this oscillation within three neutrino mixing in the Standards Model, which only allows two independent $\Delta m^{2}$ values.

In this high $\Delta m^{2}$ region, the MiniBooNE experiment recently made searches for $\nu_{e}$ [24, 25] and $\bar{\nu}_{e}$ [26] appearances in the $\nu_{\mu}$ and $\bar{\nu}_{\mu}$ beams. The MiniBooNE results excluded most of the LSND allowed region with the $\nu_{e}$ appearance measurement, however, they found the event excess with $\bar{\nu}_{e}$ appearance measurements, which are consistent with the LSND result. These results initiated further interest to the possibility of the neutrino oscillation at $\Delta m^{2} \sim 1 \mathrm{eV}^{2}$. We will discuss an interpretation of this LSND signal in the following sections.

\subsubsection{Neutrino oscillation with sterile neutrinos}

The LSND experiment observed a $3.8 \sigma$ event excess of $\bar{\nu}_{e}$ events in a $\bar{\nu}_{\mu}$ beam which can be interpreted as a signal of neutrino oscillation at $\Delta m^{2} \sim 1 \mathrm{eV}^{2}$ and $\sin ^{2} 2 \theta \sim 0.003$. A possible explanation of the signal is a mixing with additional sterile flavors of neutrino $\left(\nu_{s}\right)$ with mass eigenstates on the order of $\sim 1 \mathrm{eV}^{2}$. Here we consider the mixing with three active flavor and one $(3+1)$ or two $(3+2)$ sterile flavors, as shown in Fig. 1.2 .
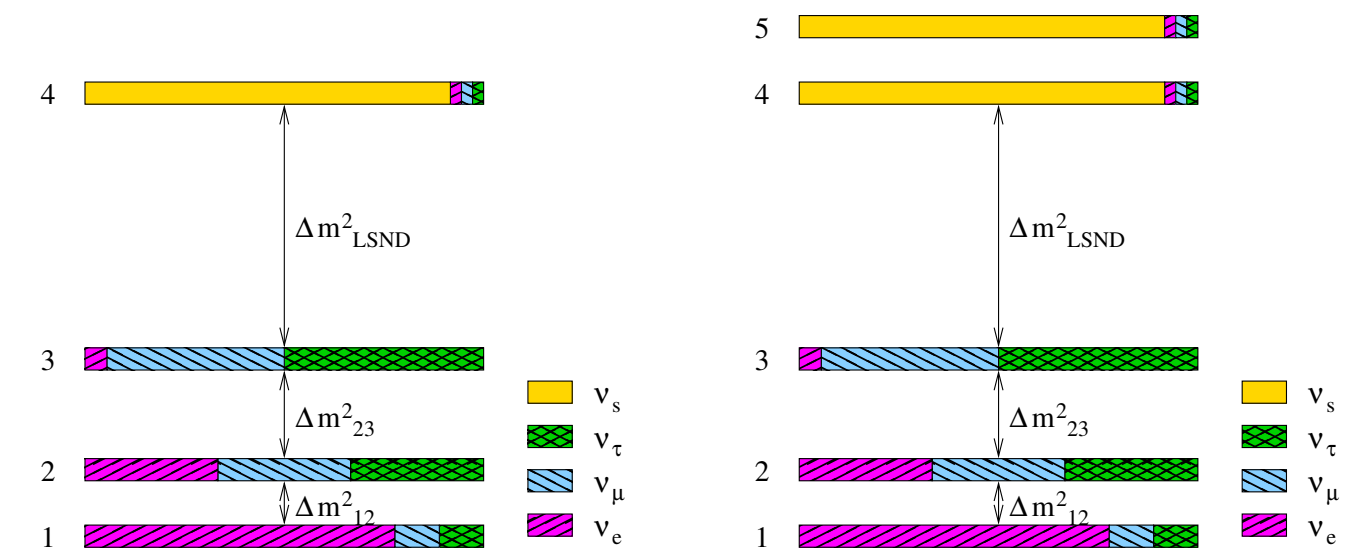

Figure 1.2: Flavor content of neutrino mass eigenstates in $(3+1)$ models (left panel) and $(3+2)$ models (right panel). The flavor contents are schematically shown.

To search for neutrino oscillations at $\Delta m^{2} \sim 1 \mathrm{eV}^{2}$, the typical neutrino beam energy is in the range between a few $\mathrm{MeV}$ and a few $\mathrm{GeV}$, and the typical baseline length is between a few meters and a few $\mathrm{km}$, since the oscillation becomes maximum with these parameters. This baseline length is relatively short compared to those of solar or atmospheric neutrino oscillation measurements. Therefore, experiments that are sensitive to sterile neutrino oscillation are referred to as shortbaseline oscillation experiments.

For short-baseline experiments, we can neglect the oscillations due to the solar and atmospheric mass splittings. Hence, we set $\Delta m_{21}^{2}=\Delta m_{32}^{2}=0$, and $\Delta m_{41}^{2}=\Delta m_{42}^{2}=\Delta m_{43}^{2}$. Then, for the $(3+1)$ model, the oscillation probability in Eq. 1.22 becomes

$$
P\left(\nu_{\alpha} \rightarrow \nu_{\beta \neq \alpha}\right)=4\left|U_{\alpha 4}\right|^{2}\left|U_{\beta 4}\right|^{2} \sin ^{2}\left[1.27 \Delta m_{41}^{2} \frac{L}{E}\right]
$$

and

$$
P\left(\nu_{\alpha} \rightarrow \nu_{\alpha}\right)=1-4\left|U_{\alpha 4}\right|^{2}\left(1-\left|U_{\alpha 4}\right|^{2}\right) \sin ^{2}\left[1.27 \Delta m_{41}^{2} \frac{L}{E}\right] .
$$

From these equations, we see that the probability of $\nu_{e}$ appearance from $\nu_{\mu}$ beam, $P\left(\nu_{\mu} \rightarrow \nu_{e}\right)$, is proportional to $\left|U_{e 4}\right|^{2}\left|U_{\mu 4}\right|^{2}$. On the other hand, $\left|U_{e 4}\right|^{2}$ and $\left|U_{\mu 4}\right|^{2}$ can be constrained by $\nu_{e}$ and $\nu_{\mu}$ disappearance $\left(P\left(\nu_{e} \rightarrow \nu_{\ell}\right)\right.$ and $\left.P\left(\nu_{\mu} \rightarrow \nu_{\mu}\right)\right)$ experiments independently. Therefore, measurements of $\nu_{e}$ and $\nu_{\mu}$ disappearance are complementary for the $\nu_{\mu} \rightarrow \nu_{e}$ appearance searches. 
Table 1.2 summarizes the results of short-baseline $\nu_{e}$ and $\nu_{\mu}$ disappearance measurements. The compatibilities of these data with the LSND and the MiniBooNE results in the context of $(3+1)$ and $(3+2)$ sterile neutrino models are extensively discussed in Refs. [27 31].

Table 1.2: Summary of short-baseline $\nu_{e}$ and $\nu_{\mu}$ disappearance measurements which provide constraints to the LSND-allowed sterile neutrino oscillation parameters [32].

\begin{tabular}{cccccc}
\hline \hline Experiment & Channel & $\begin{array}{c}\mathrm{L} / \mathrm{E} \\
{[\mathrm{km} / \mathrm{GeV}]}\end{array}$ & $\begin{array}{c}\text { Optimal } \Delta m^{2} \\
{\left[\mathrm{eV}^{2}\right]}\end{array}$ & $\begin{array}{c}90 \% \text { C.L. } \sin ^{2} 2 \theta \text { limit } \\
\text { at optimal } \Delta m^{2}\end{array}$ & Ref. \\
\hline Bugey & $\bar{\nu}_{e} \rightarrow \bar{\nu}_{\phi}$ & $2-50$ & 0.6 & $<1.3 \times 10^{-2}$ & {$[33]$} \\
CHOOZ & $\bar{\nu}_{e} \rightarrow \bar{\nu}_{\phi}$ & $100-400$ & 0.006 & $<5.0 \times 10^{-2}$ & {$[34]$} \\
CCFR & $\nu_{\mu} \rightarrow \nu_{\mu}$ & $0.004-0.03$ & 900 & $<2.0 \times 10^{-2}$ & {$[35]$} \\
CDHS & $\nu_{\mu} \rightarrow \nu_{\mu}$ & $0.02-1.5$ & 3.0 & $<5.3 \times 10^{-2}$ & {$[36]$} \\
\hline \hline
\end{tabular}

Figure 1.3 shows the results of a global fit to the current existing oscillation measurements in the $(3+1)$ sterile neutrino model [30]. Results from atmospheric neutrino oscillation measurement at SK [12], no-evidence (NEV) experiments (Bugey [33], CHOOZ [34, Palo Berde [37], CDHS [36], KARMEN [38] and NOMAD [39]), and MiniBooNE result for $E_{\nu}>475 \mathrm{MeV}$ (MB475) [24] are used for the fit. Assuming that the oscillation probability of neutrinos and anti-neutrinos are same, we see that the allowed region by the LSND experiment is excluded by the other disappearance measurements.
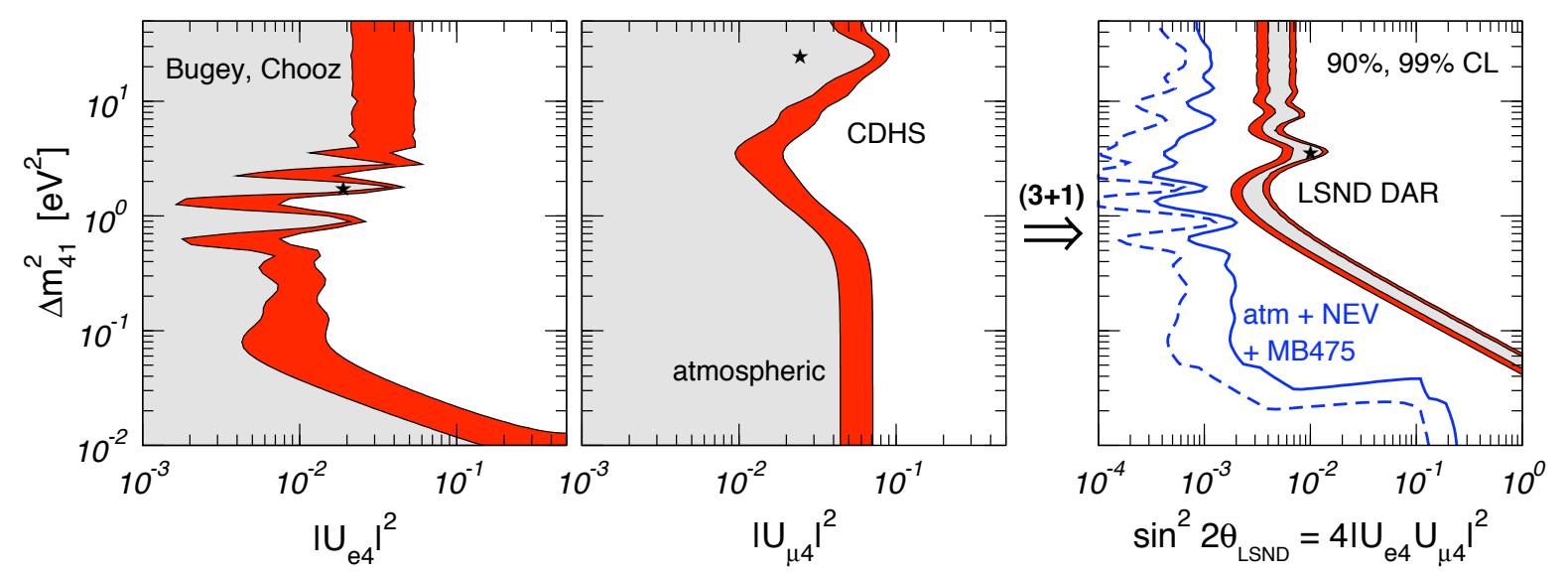

Figure 1.3: Bounds on $\left|U_{e 4}\right|^{2}$ (left panel), on $\left|U_{\mu 4}\right|^{2}$ and on $\sin ^{2} 2 \theta=4\left|U_{e 4} U_{\mu 4}\right|^{2}$ in the $(3+1)$ scheme, as a function of $\Delta m_{41}^{2}$. In the left (middle) panels, allowed regions from $\nu_{e}\left(\nu_{\mu}\right)$ disappearance experiments are shown as shaded regions. Different contours correspond to $90 \%$ and $99 \%$ C.L.. Limits obtained by a global fit to all experimental data listed in the text is shown in the right panel. The fit excludes the parameter region on the right-hand side of the blue solid (dashed) line by $90 \%$ (99\%) C.L. The allowed region from the LSND decay-at-rest (DAR) data [23] is also shown, and it is incompatible with the other data in this $(3+1)$ scheme. This figure is from Ref. [30].

With the $(3+2)$ model, the oscillation probability is given by

$$
\begin{gathered}
P\left(\nu_{\alpha} \rightarrow \nu_{\alpha}\right)=1-4\left[\left(1-\left|U_{\alpha 4}\right|^{2}-\left|U_{\alpha 5}\right|^{2}\right)\left(\left|U_{\alpha 4}\right|^{2} \sin ^{2} x_{41}+\left|U_{\alpha 5}\right|^{2} \sin ^{2} x_{51}\right)\right. \\
\left.+\left|U_{\alpha 4}\right|^{2}\left|U_{\alpha 5}\right|^{2} \sin ^{2} x_{54}\right], \\
P\left(\nu_{\alpha} \rightarrow \nu_{\beta \neq \alpha}\right)=4\left|U_{\alpha 4}\right|^{2}\left|U_{\beta 4}\right|^{2} \sin ^{2} x_{41}+4\left|U_{\alpha 5}\right|^{2}\left|U_{\beta 5}\right|^{2} \sin ^{2} x_{51} \\
+8\left|U_{\alpha 5}\right|\left|U_{\beta 5}\right|\left|U_{\alpha 4}\right|\left|U_{\beta 4}\right| \sin x_{41} \sin x_{51} \cos \left(x_{54}-\phi_{54}\right),
\end{gathered}
$$


where $x_{i j}=1.27 \Delta m_{i j}^{2} L / E$ and $\phi_{54}=\arg \left(U_{\alpha 5} U_{\beta 5}^{*} U_{\alpha 4}^{*} U_{\beta 4}\right)$.

Figure 1.4 show the results of global fits to the existing measurements at the appearance and disappearance experiments with the $(3+2)$ model. In this case, there are four parameters which describe $\nu_{\mu} \rightarrow \nu_{e}$ oscillation probability: $\left|U_{e 4} U_{\mu 4}\right|,\left|U_{e 5} U_{\mu 5}\right|, \Delta m_{41}^{2}$ and $\Delta m_{51}^{2}$. The figure shows the allowed region projected into $\left|U_{e 5} U_{\mu 5}\right|$ vs. $\left|U_{e 4} U_{\mu 4}\right|$ plane. The allowed regions by the appearance data (LSND [23], KARMEN [38], NOMAD [39] and MiniBooNE 24]) and by the disappearance data (Bugey [33], CHOOZ [34, Palo Berde [37] and CDHS [36]) are separately shown. We can see that the compatibility is slightly better than the $(3+1)$ model, but still largely incompatible each other. Hence, further search for $\nu_{\mu}$ disappearance can play a crucial role in testing these models.

There are many more exotic models to explain the LSND and other existing measurements, including sterile neutrinos in extra dimensions [40], decaying sterile neutrino [41] and CPT violation [42]. Further searches of $\nu_{\mu}$ and $\nu_{e}$ disappearance can also test these models with high precision.
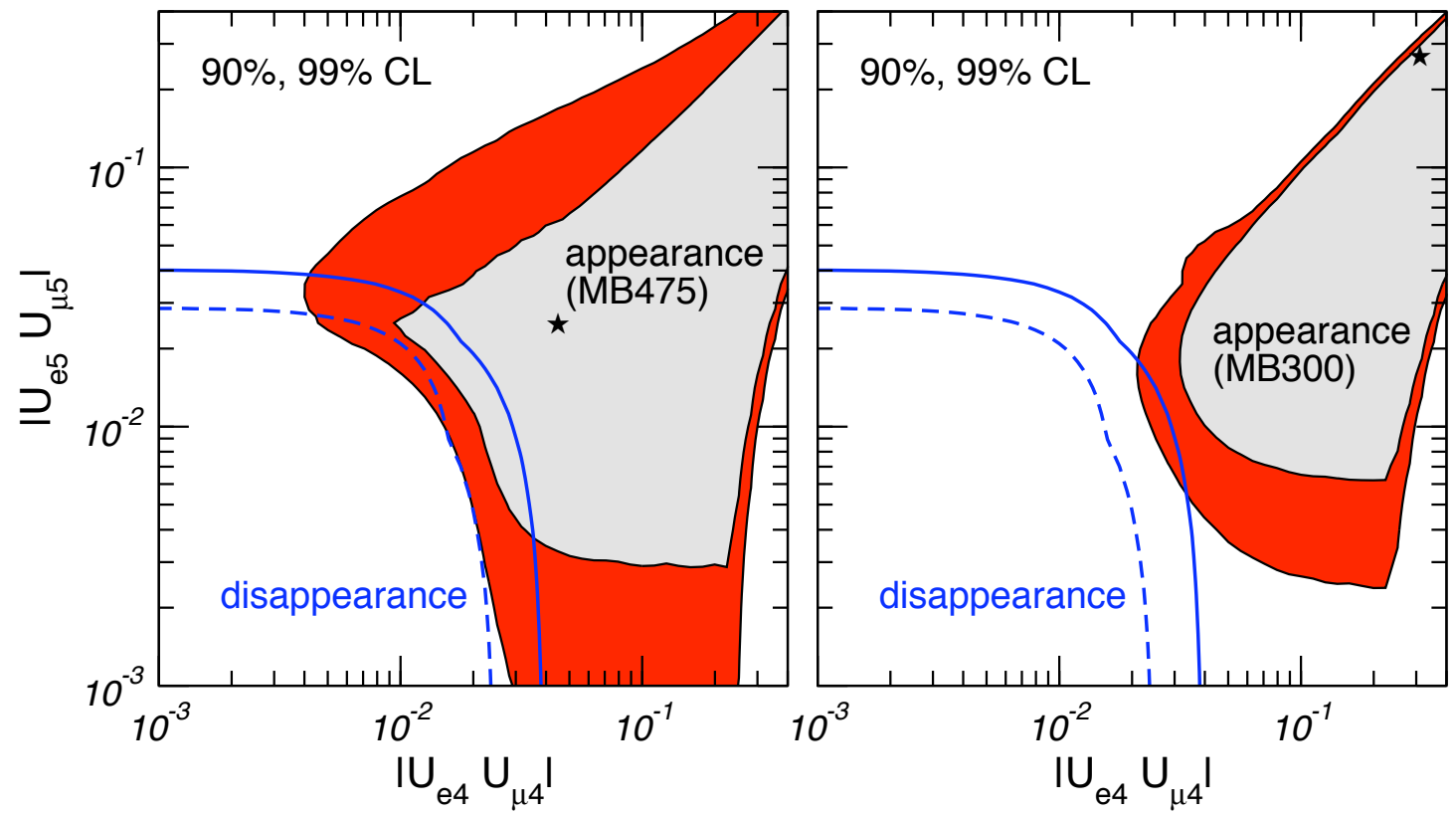

Figure 1.4: Allowed regions at $90 \%$ and $99 \%$ C.L. in $(3+2)$ schemes for appearance data (shaded regions) and disappearance data (bottom left side of the solid (90\% C.L.) and dashed (99\% C.L.) curves) projected onto the plane of $\left|U_{e 4} U_{\mu 4}\right|$ and $\left|U_{e 5} U_{\mu 5}\right|$. In the left panel MiniBooNE (MB) data with $E_{\nu}>475 \mathrm{MeV}$ (MB475) is used in the fit whereas in the right panel the full MB energy range is used in the fit (MB300). This figure is from Ref. [31].

\section{$1.4 \nu_{\mu}$ disappearance search with the Fermilab Booster Neutrino Beam}

If there is some mechanism which violates $\mathrm{CP}$ or CPT invariance, $P\left(\nu_{\alpha} \rightarrow \nu_{\beta}\right)$ is different from $P\left(\bar{\nu}_{\alpha} \rightarrow \bar{\nu}_{\beta}\right)$. This possibility become particularly interesting since MiniBooNE observed an event excess in the $\bar{\nu}_{\mu} \rightarrow \bar{\nu}_{e}$ search [26] while they find null oscillation signal in the $\nu_{\mu} \rightarrow \nu_{e}$ search [24, 25]. Hence, it is important to search for $\nu_{\mu}$ and $\bar{\nu}_{\mu}$ disappearances independently to test any possible scenarios which explain the LSND and MiniBooNE measurements.

Recently, MiniBooNE group made a search for $\nu_{\mu}$ and $\bar{\nu}_{\mu}$ disappearances with the Fermilab Booster Neutrino Beam [43], using MiniBooNE data only [44]. They obtained limits slightly bet- 
ter than CDHS experiment in $\nu_{\mu}$ disappearance measurement, and made the first search for $\bar{\nu}_{\mu}$ disappearance at $0.5<\Delta m^{2}<10 \mathrm{eV}^{2}$, as shown in Fig. 1.5.

However, this analysis is suffered by large uncertainties of the neutrino beam flux and the neutrino-nucleus interaction cross sections. Therefore the measurement could be improved if we could reduce the uncertainties by measuring the neutrino beam flux and cross sections with the front detector for MiniBooNE in the Fermilab Booster Neutrino Beam.

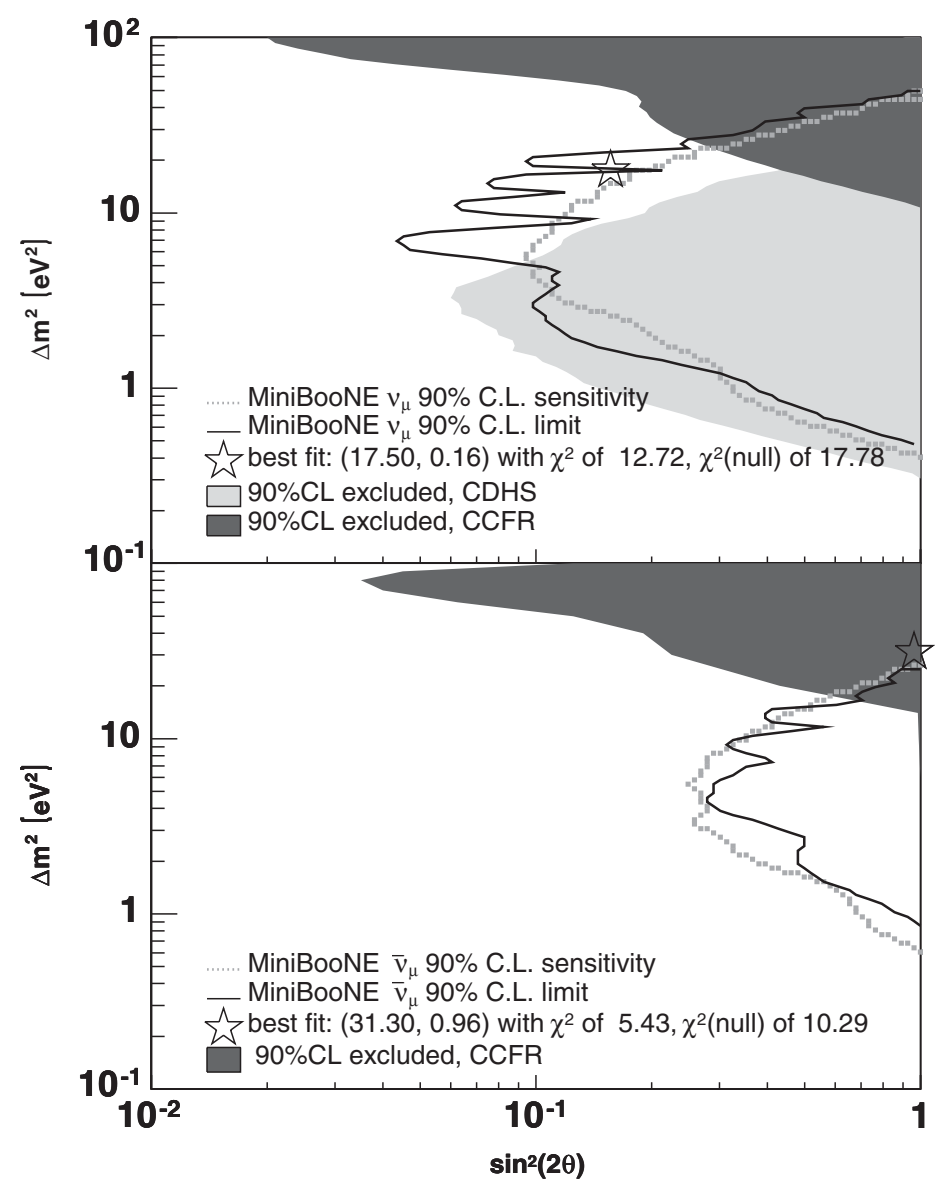

Figure 1.5: The sensitivity (dashed line) and limit (solid line) for 90\% C.L. for neutrino (top plot) and anti-neutrino (bottom plot) disappearance search with MiniBooNE data only. The righthand side of these curves are excluded. The best fit points are obtained by fits to the MiniBooNE spectrum shapes with two parameters $\left(\sin ^{2} 2 \theta, \Delta m^{2}\right)$. Excluded region by CCFR (dark grey) and CDHS (light grey) are also shown. These figures are from [44].

To test the scenario with $\mathrm{CP}$ or $\mathrm{CPT}$ violation, global fits to world existing short-baseline neutrino oscillation measurements are made for either neutrino or anti-neutrino data only [29, 45. The obtained allowed regions of global $(3+1)$ sterile neutrino fits are shown in Figure 1.6. The results from MiniBooNE measurements are also shown in this figure. Interestingly, we see allowed regions exist just below the limits obtained by the MiniBooNE. Especially, the best fit value for antineutrino data suggest a significant $\left(\sin ^{2} 2 \theta>0.2\right)$ short-baseline $\bar{\nu}_{\mu}$ disappearance, as a consequence of the observed event excess at LSND and MiniBooNE and strong $\bar{\nu}_{e}$ disappearance limits from Bugey and CHOOZ. This further motivates to improve the previous MiniBooNE measurements and to explore these allowed parameter regions.

As discussed in more detail in the rest of this thesis, we conducted the SciBooNE experiment [46] to provide the flux and cross section measurements for $\nu_{\mu}$ disappearance search for MiniBooNE, as well as to measure the neutrino interaction cross sections themselves more precisely. We report an 
improved search for $\nu_{\mu}$ disappearance by using data from both the SciBooNE and the MiniBooNE experiments in this thesis.
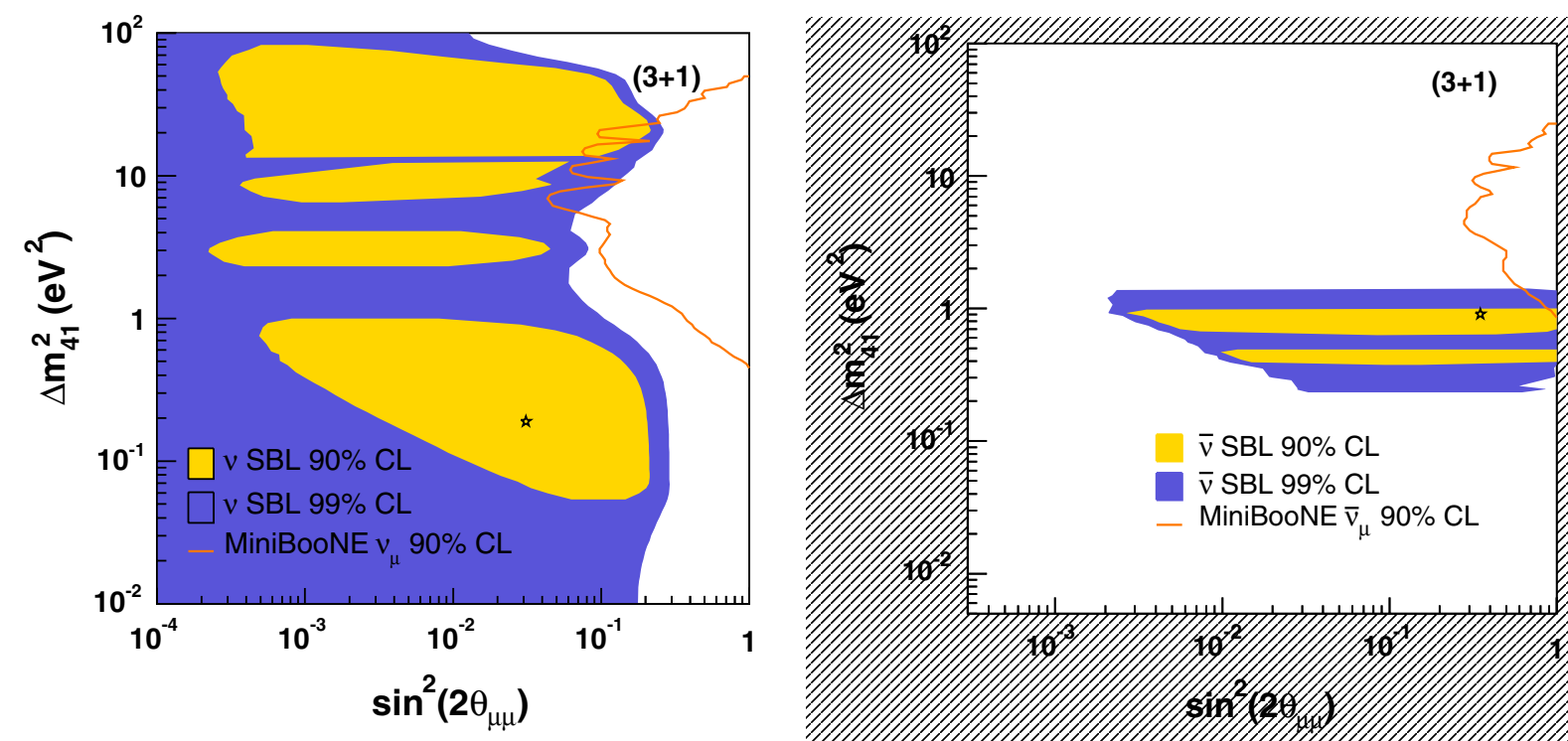

Figure 1.6: The $90 \%$ and $99 \%$ allowed regions for $\nu_{\mu}$ disappearance from a global fit to the all neutrino (left) and to the all anti-neutrino (right) data sets. The $90 \%$ C.L. limits from MiniBooNE measurements $\nu_{\mu}$ and $\bar{\nu}_{\mu}$ disappearance searches [44] are also shown. These plots are taken from Ref. [29].

\subsection{Measurement of inclusive charged current interactions}

\subsubsection{Importance of inclusive charged current interaction measurements}

As mentioned in the previous section, one of the major source which reducing the sensitivity of $\nu_{\mu}$ disappearance in MiniBooNE is the uncertainty of the neutrino interaction cross section.

In MiniBooNE, they detect muon neutrino charged current (CC) interactions on carbon in the $1 \mathrm{GeV}$ region. In this energy region, the dominant interaction process is CC quasi-elastic (QE) interaction $\left(\nu_{\mu} n \rightarrow \mu^{-} p\right)$ which is about $60 \%$ of total CC interaction, with the sub-dominant (35\%) CC single meson production $\left(\nu_{\mu} N \rightarrow \mu^{-} N^{\prime} m\right)$ where $N$ and $N^{\prime}$ are the nucleons (proton or neutron) and $m$ is the meson. For the $\nu_{\mu}$ disappearance analysis, they select CC-QE interactions and reconstruct the neutrino energy from the muon kinematics, as described in more detail in Appendix C.

The major uncertainties of the neutrino-nucleus interaction are categorized into the following three components:

- Neutrino-nucleon interaction model.

- Nuclear model (Fermi motion and nuclear potential).

- Intra-nuclear interactions of the hadronic final states.

The uncertainty of the neutrino-nucleon interaction is predominantly due to the uncertainty of the axial form factor (known as $F_{A}$ [47]) of the nucleons, which can not be measured by electron scattering experiments and hence can be determined by neutrino interactions only. This axial form factor changes both the absolute normalization and the muon kinematics. As for the normalization, we have $\sim 20 \%$ error associated with the $F_{A}$ uncertainty. 
Also, the uncertainty of the nuclear model is significant especially in the $1 \mathrm{GeV}$ region, because the Fermi surface momentum of carbon nucleus, which is about $200 \mathrm{MeV}$, is close to the neutrino beam energy. The most significant feature of this nuclear effect is the Pauli-blocking, causing the suppression of the small $Q^{2}$ interactions. Recently, MiniBooNE collaboration measured CC-QE interactions and found a significant event deficit at the low- $Q^{2}$ region [48. This indicates that the current nuclear model with the relativistic Fermi gas model [49] dose not describe well the experimental data. Hence, we have large uncertainty associated with this.

Finally, to select an exclusive interaction mode (like CC-QE), one need to detect hadronic final states in addition to the muons. However, there is a large uncertainty of the intra-nuclear interactions of the hadronic final states (e.g. nucleon re-scattering and pion charge exchanges). We often refer this as to the final state interactions (FSI). Hence, for exclusive interaction measurements, we have a large uncertainty of the background contamination due to the uncertainties of the FSI.

These uncertainties all affected to the oscillation measurements at MiniBooNE, as well as to any other experiment uses neutrino-nucleus interactions in the few $\mathrm{GeV}$ region. Hence, we need to know these cross sections precisely. However, the exclusive interaction cross sections are always suffered by the large uncertainty of the FSI. Therefore, it is very important to measure inclusive cross sections as well, and compare with the exclusive measurements.

Figure 1.7 shows the summary of current existing neutrino CC interaction cross section measurements. In addition, NOMAD [50] and MINOS [51] recently reported more precise CC inclusive interaction cross section measurements in the energy region above $\sim 3 \mathrm{GeV}$. In the $\sim 1 \mathrm{GeV}$ region, where the recent oscillation experiments are conducted, all inclusive CC measurements were made on deuterium targets using old bubble chamber experiments [52, 53]. As mentioned before, the nuclear effects of the neutrino target material are significant in the $1 \mathrm{GeV}$ region. Therefore, the cross sections on deuterium targets are not directly applicable to the heavier nuclear target materials used in the recent accelerator-based neutrino experiments in this energy region, e.g. SciBooNE, MiniBooNE and T2K. Furthermore, the results in Ref. [53] use the CC quasi elastic (QE) interactions to normalize the absolute neutrino flux, which introduces additional ambiguity caused by the choice of $\mathrm{CC}-\mathrm{QE}$ interaction models. Hence, the $\mathrm{CC}$ inclusive interaction cross section on nuclear targets in the $1 \mathrm{GeV}$ region is a large missing piece.

\subsubsection{Benefits to the measurements of exclusive channels}

There are several recent exclusive cross section measurements awaiting for comparison with the CC inclusive interaction cross sections.

The first one is the CC-QE cross section measurement on carbon at $0.4<E_{\nu}<2.0(\mathrm{GeV})$ by MiniBooNE [66], shown in Figure 1.8. In thise figure, $M_{A}$ is the axial mass for the dipole axial form factor, $F_{A}\left(Q^{2}\right)=g_{A} /\left(1+\left(Q^{2} / M_{A}^{2}\right)\right)$, where $g_{A}=-1.267$ is determined from neutron $\beta$-decay measurements, and $\kappa$ is an empirical parameter to tune the size of Pauli-blocking where $\kappa=1.0$ is the default value. Interestingly, the obtained cross section is higher than what extrapolated from a measurement at $E_{\nu}>3(\mathrm{GeV})$ by NOMAD [67, and obtained $M_{A}$ values are incompatible. Then, this discrepancy could be due to the uncertainty of the nuclear model, or the uncertainty of the FSI which lead to mis-estimate the backgrounds from CC single meson productions. To check the effect of the FSI, we need a measurement of CC inclusive interaction in MiniBooNE energy region, ideally, as well as in the intermediate energy region at $2.0<E_{\nu}<3.0(\mathrm{GeV})$.

The second is the recent cross section measurements by the SciBooNE collaboration. We measured the ratio of the cross sections of charged current coherent pion production [68] and neutral current neutral pion production 69, 70, which were both normalized to CC inclusive interactions. The CC pion production and NC pion production are the the largest backgrounds for $\nu_{\mu}$ disappearance and $\nu_{e}$ appearance measurements, respectively. Hence, these are especially important for the neutrino oscillation experiments in the $1 \mathrm{GeV}$ region, such as MiniBooNE and T2K. However, these results are usually hard to compare with the cross section models since the models usually predict 


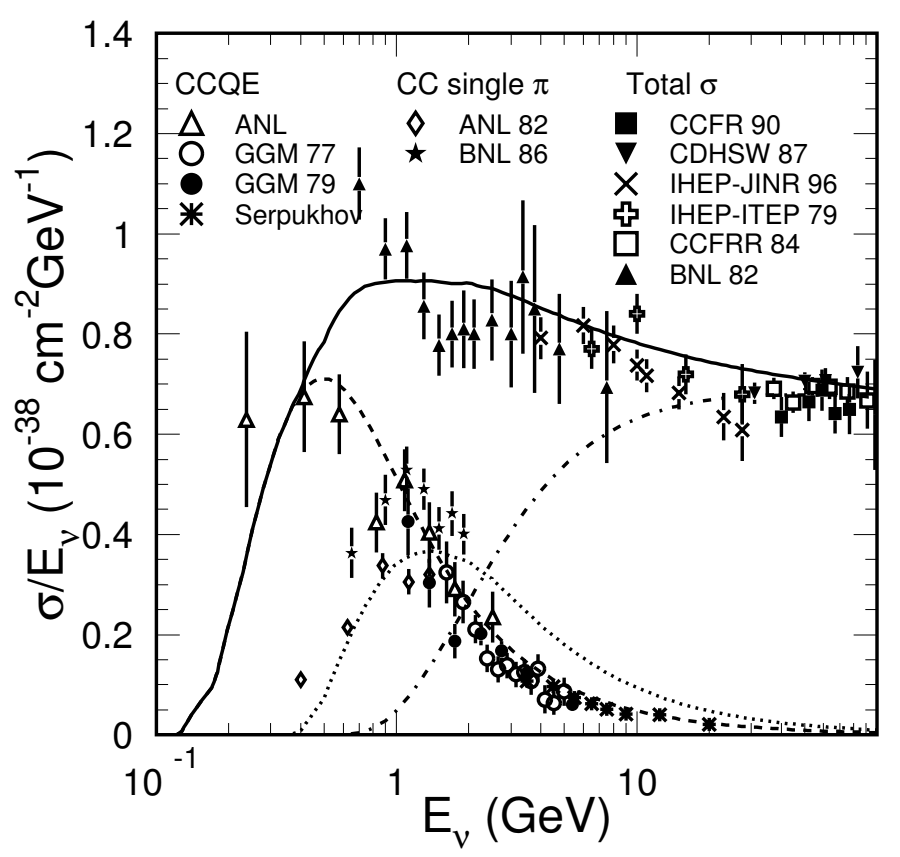

Figure 1.7: Charged current total cross sections divided by neutrino energy for neutrino nucleon charged current interactions. The solid line shows the calculated total cross section. The dashed, dot and dash-dotted lines show the calculated quasi-elastic, single-meson and deep-inelastic scatterings, respectively. The data points are taken from the following experi-

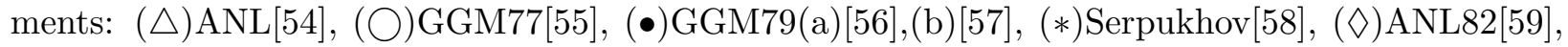

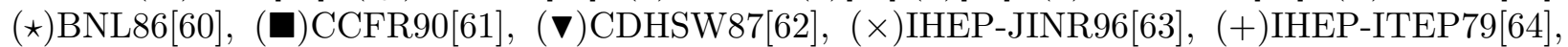
$(\square)$ CCFRR84[65], and ( $\mathbf{\Delta}$ BNL82[53].

the absolute cross sections, not the ratios to the total CC interaction cross section. Once the absolute normalization for these measurements are given by a CC inclusive cross section measurement, precise comparisons with the theoretical predictions become possible.

Because of poor knowledge of cross sections, several neutrino interaction simulators are often used and tested to predict the different cross sections and the kinematics of final state particles. Among these, NEUT [71, 72] and NUANCE [73] are commonly used for the recent neutrino oscillation and interaction measurements. NEUT is used in the Kamiokande [74, Super-Kamiokande [12], K2K [13, and T2K experiments, while NUANCE is used in MiniBooNE and as a check of simulations in several experiments. Although they are both tuned to describe the experimental data, they have not yet been precisely tested and compared with each other in a single experiment. Hence, in order to make a precise measurement of neutrino oscillations, it is important to test these cross section models together and to select models which well describe experimental data.

\subsection{Overview of this thesis}

In this thesis, we report on a measurement of CC inclusive interactions of muon neutrinos in the few $\mathrm{GeV}$ region. We also use the measurement to improve a search for short-baseline muon neutrino disappearance with the Fermilab Booster Neutrino Beam.

We collected high-statistics CC interaction sample at SciBooNE, and extracted energy dependent charged current interaction rates and cross sections for a wide energy range from $0.25 \mathrm{GeV}$ to $\sim 3 \mathrm{GeV}$. This is the first measurement of the $\mathrm{CC}$ inclusive interaction cross section on carbon 


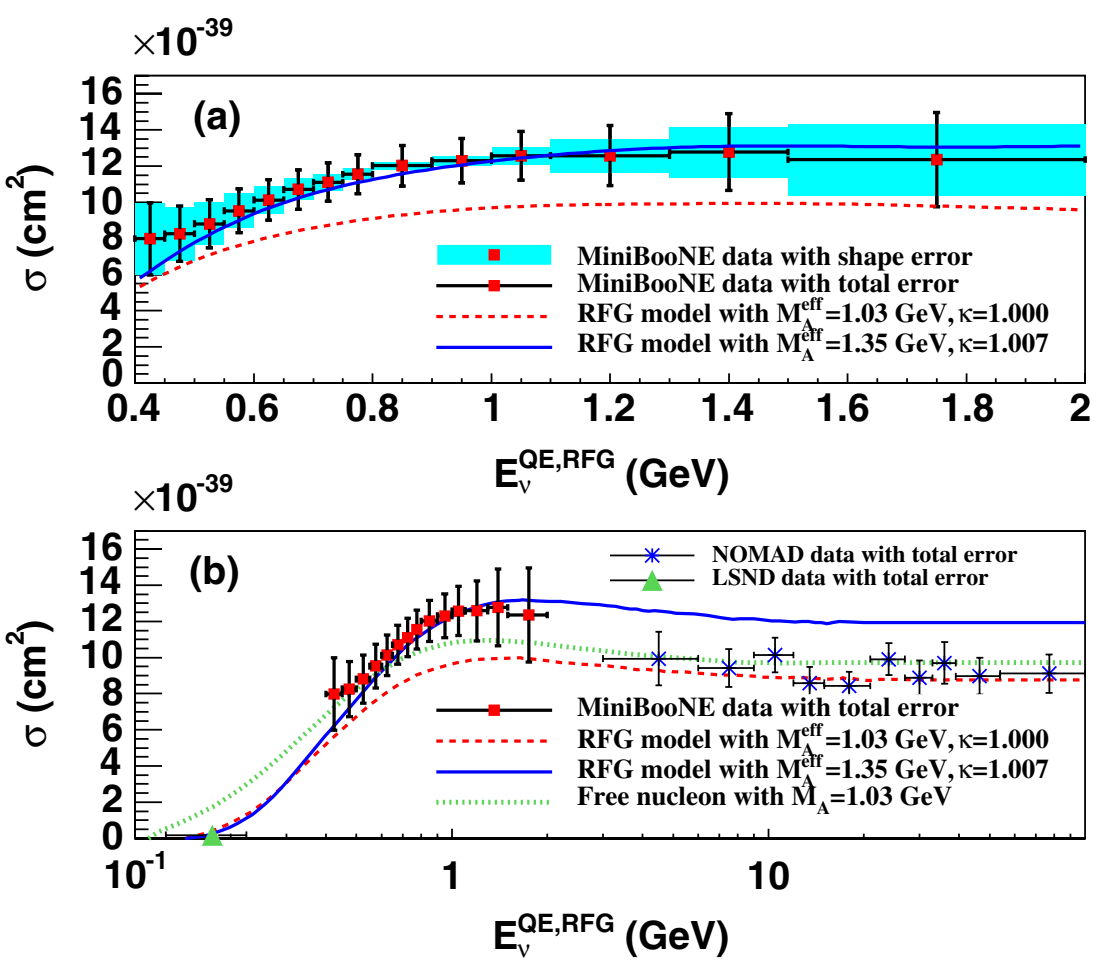

Figure 1.8: The $\nu_{\mu}$ CC-QE cross sections as a function of reconstructed neutrino energy assuming CC-QE kinematics $\left(E_{\nu}^{Q E}\right)$. The relativistic Fermi gas model (RFG) is assumed and hence the superscript "RFG" is added as $E_{\nu}^{Q E, R F G}$. These plots are taken from Ref. [66].

around $1 \mathrm{GeV}$. The results of this measurement are used for the the following three purposes.

First, the obtained CC interaction rate and cross section can be used to tune either neutrino flux and interaction models. Thus, it helps future neutrino oscillation experiments, such as T2K. We simulate neutrino interactions in SciBooNE using both NEUT and NUANCE program libraries. In this thesis, we discuss the difference of these two simulators, and report the detailed comparison with our measurements. The another unique feature of this analysis is that the result covers a wide energy range from $0.25 \mathrm{GeV}$ to $\sim 3 \mathrm{GeV}$. This also give an important information to test the energy dependence of various cross section models, and to test the discrepancy between MiniBooNE and NOMAD measurements.

Second, this analysis provides the normalization for SciBooNE's previous cross section ratio measurements for charged current coherent pion production [68] and neutral current neutral pion production [69, 70]. This thesis provides an CC interaction cross section with the same definition used in the previous analyses, so that the measured ratios can be converted to absolute cross sections. Then, precise comparison with various cross section models become possible.

Finally, this analysis result is an important input for a search for $\nu_{\mu}$ disappearance search with the Fermilab Booster Neutrino Beam. The measured CC interaction rate is used to constrain the flux and cross section at MiniBooNE. An improved search for $\nu_{\mu}$ disappearance using both SciBooNE and MiniBooNE data is also reported in this thesis.

The remainder of this thesis is organized as follows. First, we give a brief overview of the experimental setup and the strategy of the analysis in Chapter 2, A detailed description of the experimental apparatus and the corrected data sets are given in Chapter 3 . The simulation of the SciBooNE experiment is described in Chapter 4. Then, we describe reconstruction of CC events in SciBooNE in Chapter 5. In Chapter 6, we report a measurement of CC event rate and cross section at SciBooNE. Finally, a search for $\nu_{\mu}$ disappearance using both SciBooNE and MiniBooNE data is presented in Chapter 7. The conclusions are given in Chapter 8 . 


\section{Chapter 2}

\section{Experimental technique}

In this chapter, we first introduce an overview of the experimental setups of the SciBooNE and MiniBooNE experiments. Then, we describe the principle and the strategy for the neutrino oscillation and cross section measurements.

\subsection{Overview of the experimental setup}

Figure 2.1 show the schematic drawing the experimental setup of SciBooNE and MiniBooNE experiments. The experiments use the Booster Neutrino Beam (BNB) at Fermi National Accelerator Laboratory (FNAL). The BNB produces an intense muon neutrino and anti-neutrino beam with the mean energy of $\sim 0.8 \mathrm{GeV}$. The produced neutrinos are detected by the SciBooNE and MiniBooNE detectors, which both placed on the axis of the beamline.

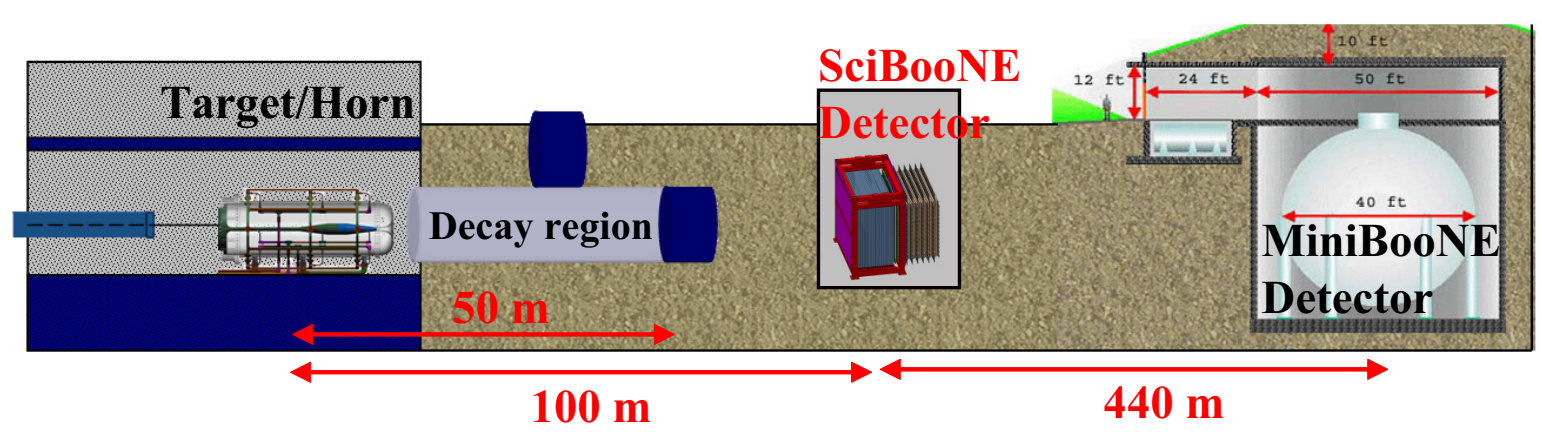

Figure 2.1: Schematic drawing of the experimental setup of SciBooNE and MiniBooNE.

\subsubsection{SciBooNE experiment}

The SciBooNE detector is positioned $100 \mathrm{~m}$ downstream from the neutrino production target, and $440 \mathrm{~m}$ upstream from the MiniBooNE detector. SciBooNE uses the Scintillator Bar (SciBar) detector, a fully active fine segmented tracking detector, which was originally developed and used for the K2K experiment [75]. SciBar acts as the neutrino target, and also can detect all charged particles produced by neutrino interactions. We started beam data taking in June 2007, and completed in August 2008.

The main physics motivations of SciBooNE are to make precise measurement of neutrino-nucleus interaction cross sections, and to act as a front detector for MiniBooNE for neutrino oscillation studies.

Figure 2.2 show the $\nu_{\mu}$ energy spectrum at SciBooNE compared with those at K2K and T2K. Since the entire range of the T2K energy spectrum is covered within the spectrum of SciBooNE, 
various neutrino cross section measurements at SciBooNE could help neutrino oscillation studies in $\mathrm{T} 2 \mathrm{~K}$.

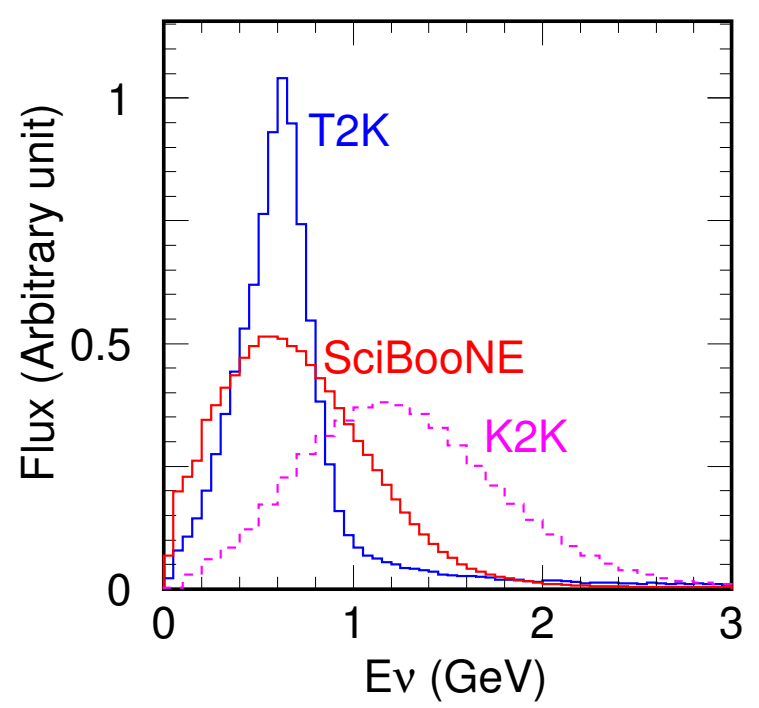

Figure 2.2: Comparison of the muon neutrino energy spectra at K2K, T2K, and SciBooNE. All curves are normalized to unit area.

As for the oscillation studies with MiniBooNE, SciBooNE can provide a constraint on the product of flux and cross-section. As shown in Fig. 2.1, the SciBooNE detector is sharing the same neutrino beam with MiniBooNE. In addition, the neutrino target materials are both essentially carbon; polystyrene $\left(\mathrm{C}_{8} \mathrm{H}_{8}\right)$ for SciBooNE, and mineral oil $\left(\mathrm{CH}_{2}\right)$ for MiniBooNE. Therefore, most of the flux and cross section uncertainties cancel when we compare the data from the two experiments. Then, a $\nu_{\mu}$ disappearance search with high sensitivity becomes possible.

\subsubsection{MiniBooNE experiment}

The MiniBooNE experiment is designed to test the $\nu_{\mu} \rightarrow \nu_{e}$ oscillation indicated by the LSND experiment. As shown in Eq. 1.22, the oscillation probability is a function of the flight length divided by the energy of neutrinos $(L / E)$. Since the observed excess of $\bar{\nu}_{e}$ events at LSND peaks at $L / E \sim 0.7 \mathrm{~m} / \mathrm{MeV}$ [23], the baseline length of MiniBooNE is set to hold this $L / E$ ratio, i.e.,

$$
\frac{L_{\mathrm{MiniBooNE}}}{E_{\mathrm{MiniBooNE}}}=\frac{\sim 540(\mathrm{~m})}{\sim 800(\mathrm{MeV})} \sim 0.7(\mathrm{~m} / \mathrm{MeV}) .
$$

The experiment started beam data taking in 2002, and still correcting data to the present.

\subsection{Principle of the measurements}

\subsubsection{Neutrino oscillation analysis}

To search for neutrino oscillation, we count the number of neutrino interactions in the detector and compare that with the prediction. The number of detected neutrino interaction $(N)$ can be written as the product of the neutrino flux $(\Phi)$, the cross section $(\sigma)$ and the detection efficiency $(\epsilon)$ as

$$
N=\Phi \cdot \sigma \cdot \epsilon
$$

However, in our current knowledge, the uncertainties of both the neutrino flux $(\Phi)$ and the neutrino interaction cross section $(\sigma)$ are large and they are only known with 10-20\% accuracy. That was 
the reason why these flux and cross section uncertainty was limiting the sensitivity of the previous $\nu_{\mu}$ disappearance search [44].

Then, we constrain these flux and cross section uncertainties using SciBooNE measurements. However, it is hard to extract flux or cross section itself with high precision, because the observed number of events is always the product of them, and the uncertainties of both flux and cross section are large. Hence, we extract the interaction "rate", $\mathcal{R}=\Phi \cdot \sigma$, which measured as

$$
\mathcal{R}_{S B}=\frac{N_{S B}^{o b s}}{\epsilon_{S B}},
$$

where $N_{S B}^{o b s}, \epsilon_{S B}$ and $\mathcal{R}_{S B}$ are observed number of events, detection efficiency and extracted interaction rate at SciBooNE, respectively. Since SciBooNE and MiniBooNE share the neutrino beam and use the same neutrino interaction target, we can assume for the first order that

$$
\Phi_{M B}=\alpha \cdot \Phi_{S B}, \quad \sigma_{M B}=\sigma_{S B},
$$

and hence,

$$
\mathcal{R}_{M B}=\alpha \cdot \mathcal{R}_{S B}
$$

where $\alpha$ is a constant number, and $\Phi_{S B(M B)}, \sigma_{S B(M B)}$ and $\mathcal{R}_{S B(M B)}$ are the neutrino flux, cross section and interaction rate at SciBooNE (MiniBooNE) detector, respectively. Then, we make a constrained prediction of the number of events in MiniBooNE $\left(M_{M B}^{p r e d}\right)$ as,

$$
M_{M B}^{\text {pred }}=\mathcal{R}_{M B} \cdot \epsilon_{M B}=\alpha \cdot \mathcal{R}_{S B} \cdot \epsilon_{M B}=\alpha \cdot \frac{N_{S B}^{o b s}}{\epsilon_{S B}} \cdot \epsilon_{M B},
$$

where $\epsilon_{M B}$ is the detection efficiency at MiniBooNE. An oscillation analysis is conducted by comparing this $M_{M B}^{\text {pred }}$ with the observed number of events, $M_{M B}^{o b s}$. This procedure is schematically drawn in Figure 2.3 .

The uncertainty of the prediction, $M_{M B}^{\text {pred }}$, is now just resulting from the statistical fluctuation of the SciBooNE measurement $\left(N_{S B}^{o b s}\right)$, the detection efficiencies $\left(\epsilon_{S B}, \epsilon_{M B}\right)$, and the SciBooNEMiniBooNE flux ratio $(\alpha)$. With a high precision and high statistics measurement at SciBooNE, we can make these errors much smaller than the original error, which resulting from the flux and cross section uncertainties. Then, we make an improved search for $\nu_{\mu}$ disappearance with this setup.

Measurement at SciBooNE

Prediction at MiniBooNE

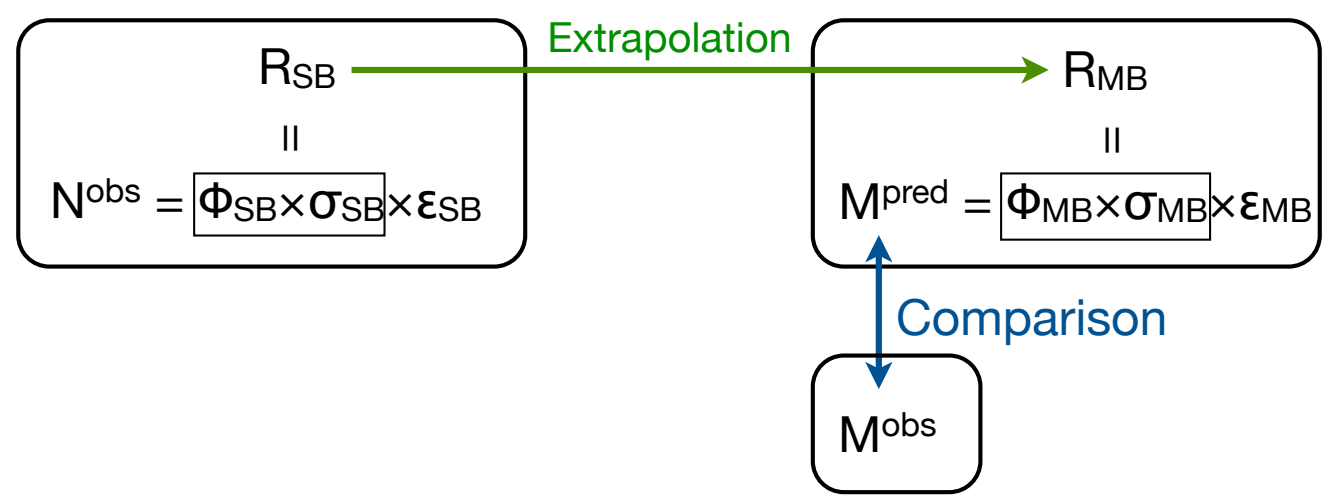

Observation at MiniBooNE

Figure 2.3: A simple flow of the SciBooNE-MiniBooNE joint oscillation analysis. 
In practice, we extract the rate normalization factor, $f=\mathcal{R}_{S B} / \mathcal{R}_{S B}^{\text {orig }}$, from the SciBooNE data. Here, $\mathcal{R}_{S B(M B)}^{\text {orig }}$ is the original prediction of the neutrino interaction rate at SciBooNE(MiniBooNE). Then, the prediction of the number of MiniBooNE events is calculated as

$$
M_{M B}^{\text {pred }}=f \cdot \mathcal{R}_{M B}^{\text {orig }} \cdot \epsilon_{M B}\left(=\frac{\mathcal{R}_{S B}}{\mathcal{R}_{S B}^{\text {orig }}} \cdot \mathcal{R}_{M B}^{\text {orig }} \cdot \epsilon_{M B}=\alpha \cdot \mathcal{R}_{S B} \cdot \epsilon_{M B}\right) .
$$

\subsubsection{Measurement of neutrino interaction cross section}

Using the measured interaction rate at SciBooNE, we also extract the interaction cross section by using the predicted flux,

$$
\sigma=\frac{\mathcal{R}_{S B}}{T \cdot \Phi_{S B}}
$$

where $T$ is the number of target nucleons in the SciBooNE detector. For the cross section calculation, additional uncertainty of the number of target $(T)$ and the flux prediction $\left(\Phi_{S B}\right)$ are included. Hence, the total uncertainty is larger than that of the rate measurement. However, this cross section is an important information to compare with the theoretical predictions of the neutrino interactions.

\subsection{Analysis strategy}

Figure 2.4 show the a simple flow of the analysis strategy presented in this thesis. The first step is that we extract energy dependent neutrino interaction rates from SciBooNE charged current data sample, using a method called "spectrum fit". Then, the result of the rate measurement is used in two ways. First, we make the first measurement of CC inclusive cross section on carbon in the $1 \mathrm{GeV}$ region. With this result, various comparison with the theoretical predictions and exclusive channels can be made. Second, we extrapolate the rate to MiniBooNE, and make a prediction of the reconstructed $E_{\nu}$ distribution. By comparing this prediction with the observed data at MiniBooNE, we make an improved search for $\nu_{\mu}$ disappearance at $0.5<\Delta m^{2}<30\left(\mathrm{eV}^{2}\right)$.

\section{SciBooNE data}

$\downarrow$ Spectrum fit

\section{$\mathrm{CC}$ interaction rate measurement}
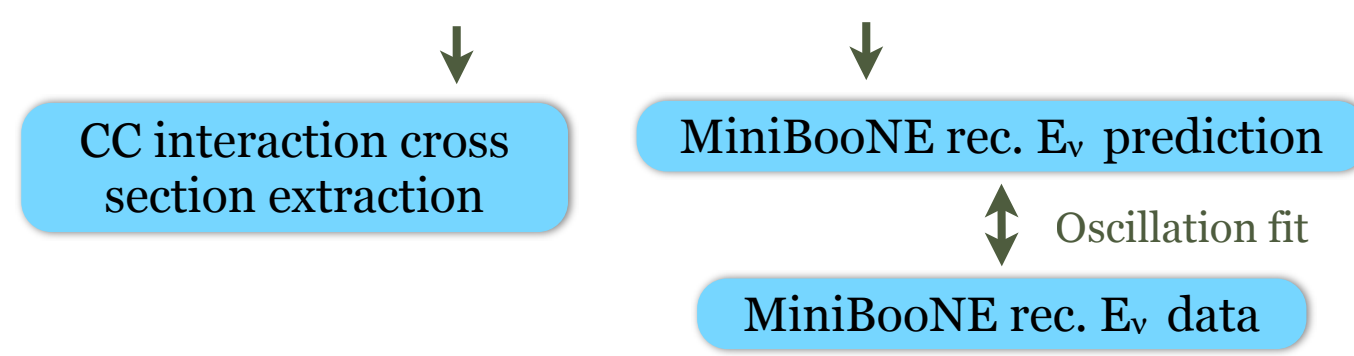

Figure 2.4: A simple flow chart of the analysis strategy 


\section{Chapter 3}

\section{Experimental apparatus and the data sets}

In this chapter, we describe the experimental apparatus, and the collected data sets of the SciBooNE and MiniBooNE experiments.

\subsection{Experimental apparatus}

\subsubsection{Booster Neutrino Beam}

Fermilab Booster accelerates the protons up to $8 \mathrm{GeV}$ kinetic energy. Selected spills containing approximately $4-5 \times 10^{12}$ protons are extracted and bent toward the BNB target hall. Each spill contains 81 bunches of protons, approximately 6 nsec wide each and $19 \mathrm{nsec}$ apart, for a total spill duration of $1.6 \mu \mathrm{sec}$. The typical beam alignment and divergence, measured by the beam position monitors located near the target, are within $1 \mathrm{~mm}$ and $1 \mathrm{mrad}$ of the nominal target center and axis direction, respectively. The typical beam focusing on target measured by the beam profile monitors is of the order of 1-2 mm (RMS) in both the horizontal and vertical directions. The number of protons delivered to the BNB target for each spill is measured with a $2 \%$ accuracy using two toroidal current transformers (often referred to as toroid's) located near the target along the beamline. These parameters are well tuned within the experiment requirements.

\subsubsection{Target and magnetic focusing horn}

The primary proton beam smashes a thick beryllium target located in the BNB target hall. Secondary mesons (pions and kaons) are produced by hadronic interactions of the protons with the target. The target is made of seven cylindrical slugs with a radius of $0.51 \mathrm{~cm}$, for a total target length of $71.1 \mathrm{~cm}$, or about 1.7 inelastic interaction lengths. The target is surrounded by a magnetic focusing horn, focusing the positively-charged secondary particles from the target to the direction pointing to the SciBooNE and MiniBooNE detectors. The magnetic horn used in the BNB is shown in Fig. 3.1. Such positively-charged secondary particles are dominated by charged pions $\left(\pi^{+}\right)$producing the neutrino beam via their decay $\left(\pi^{+} \rightarrow \mu^{+} \nu_{\mu}\right)$. The focusing is produced by the toroidal magnetic field present in the air volume between the horn's two coaxial conductors made of aluminum alloy. The horn current pulse is approximately a half-sinusoid of amplitude $174 \mathrm{kA}, 143 \mu \mathrm{sec}$ long, synchronized to each beam spill. The measured strength of the magnetic field is shown in Fig. 3.2. The polarity of the horn current flow can be (and has been) switched, in order to focus negatively-charged mesons, and therefore to produce an anti-neutrino beam instead of a neutrino beam. 


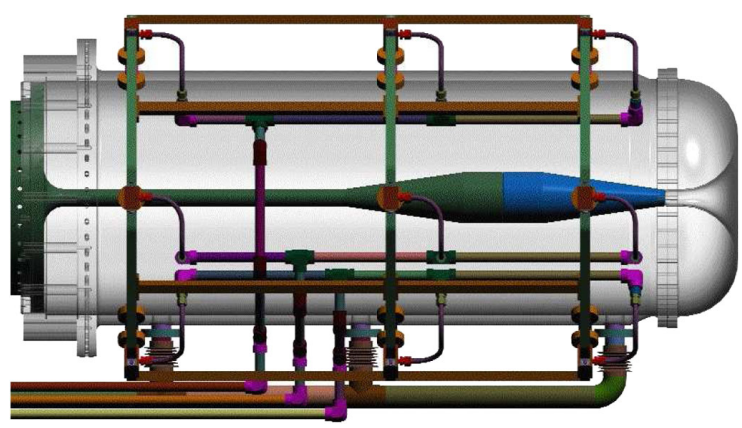

Figure 3.1: The MiniBooNE pulsed horn system. The outer conductor (gray) is transparent to show the inner conductor components running along the center (dark green and blue). The target assembly is inserted into the inner conductor from the left side. In neutrinofocusing mode, the (positive) current flows from left-to-right along the inner conductor, returning along the outer conductor. The plumbing associated with the water cooling system is also shown. This figure is from [43].

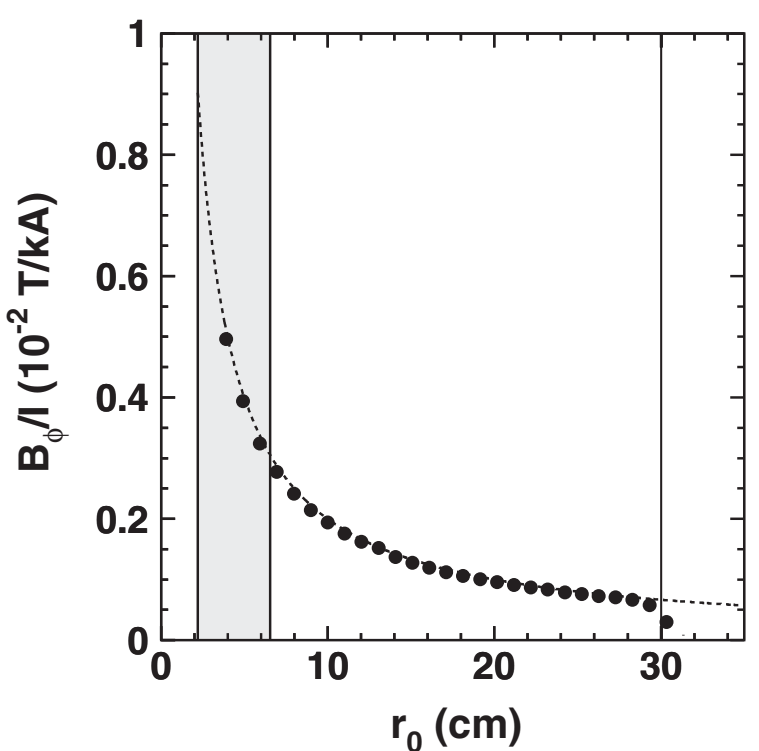

Figure 3.2: Measurements of the azimuthal magnetic field within the horn. The points show the measured magnetic field, while the line shows the expected $1 / \mathrm{R}$ dependence. The black lines indicate the minimum and maximum radii of the inner conductor. This figure is from [43].

\subsubsection{Decay region and absorber}

Figure 3.3 shows the layout of the BNB. The secondary mesons from the target/horn region are further collimated via passive shielding, and moved to a cylindrical decay region where the secondary mesons can decay into neutrinos. The decay region is filled with air at atmospheric pressure, $50 \mathrm{~m}$ long and $90 \mathrm{~cm}$ in radius. A beam absorber located at the end of the decay region stops hadronic particles and muons, and only a pure neutrino beam pointing toward the detector remains, mostly from $\pi^{+} \rightarrow \mu^{+} \nu_{\mu}$ decays.

\subsubsection{SciBooNE detector}

The SciBooNE detector is located $100 \mathrm{~m}$ downstream from the beryllium target on the axis of the beam, as shown in Figure 2.1. The detector comprises three sub-detectors: a fully active and finely segmented scintillator tracker (SciBar), an electromagnetic calorimeter (EC), and a muon range detector (MRD). SciBar is the primary neutrino target for this analysis.

SciBooNE uses a right-handed Cartesian coordinate system in which the $z$ axis is the beam direction and the $y$ axis is the vertical upward direction. The origin is located on the most upstream surface of SciBar in the $z$ dimension, and at the center of the SciBar scintillator plane in the $x$ and $y$ dimensions. Since each sub-detector is read out both vertically and horizontally, two views are defined: top ( $x$ vs. $z$ projection) and side ( $y$ vs. $z$ projection).

\subsubsection{Scintillator bar tracker (SciBar)}

The SciBar detector is located at the upstream of the other sub-detectors. The most important role of SciBar is to reconstruct the neutrino-nucleus interaction vertex and detect charged particles produced by neutrino interactions. In addition, SciBar can identify particles based on energy deposition per unit length. SciBar was originally designed and built as a near detector for the K2K 


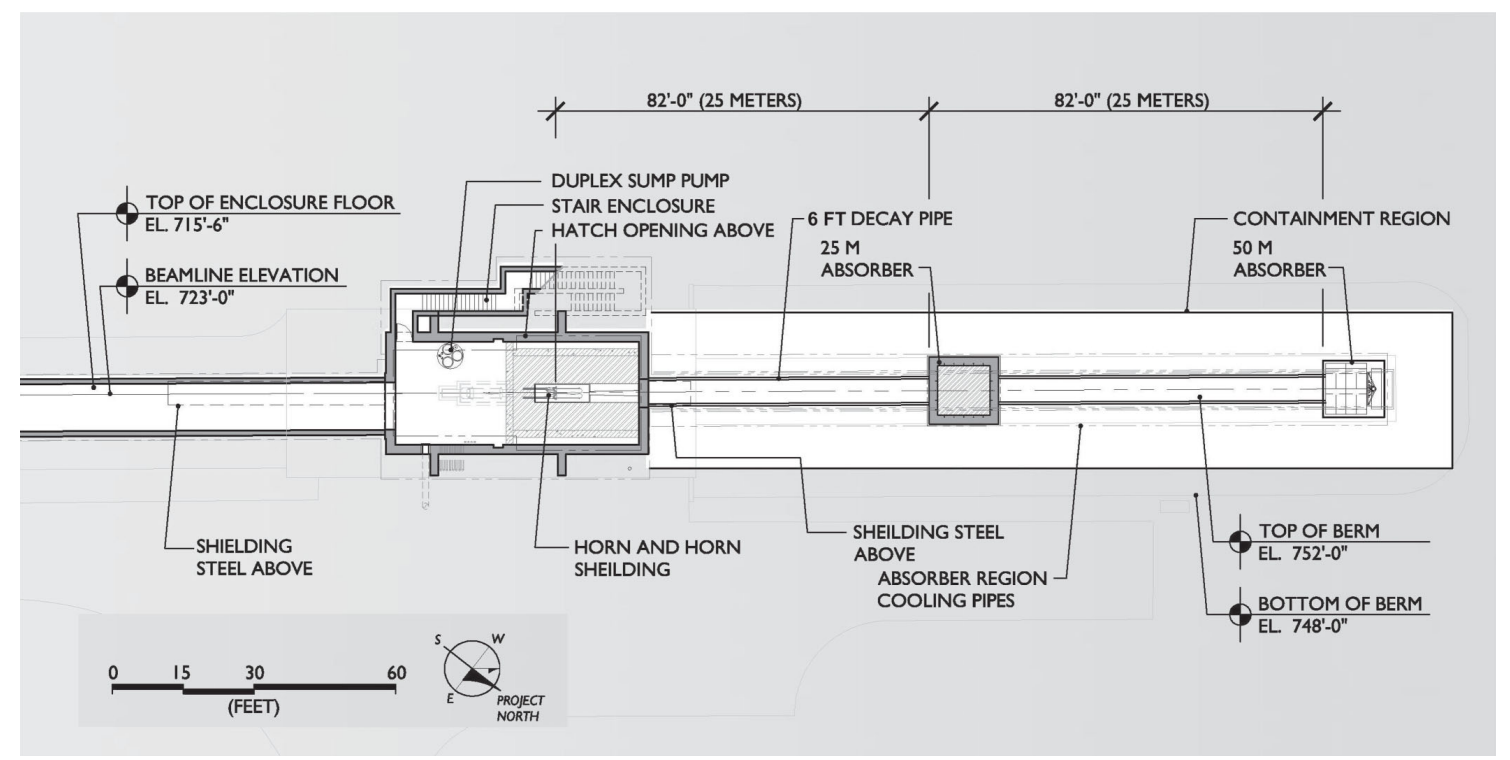

Figure 3.3: Overall layout of the BNB. The primary proton beam, extracted from the booster, enters the target hall from the left. Upon exiting the target hall, particles encounter a 50-meter-long decay region, terminating in the beam stop on the right. This figure is from Ref. [43].

experiment 75]. After K2K was completed, the SciBar detector was once disassembled, shipped to FNAL, and then re-built there for SciBooNE.

Figure 3.4 shows a schematic drawing of SciBar. The SciBar detector consists of 14,336 extruded plastic scintillator strips which serve as the target for the neutrino beam as well as the active detection medium. Each strip has dimensions of $1.3 \times 2.5 \times 300 \mathrm{~cm}^{3}$. The scintillators are arranged vertically and horizontally to construct a $3 \times 3 \times 1.7 \mathrm{~m}^{3}$ volume with a total mass of 15 tons. The dominant component of the SciBar detector is polystyrene $\left(\mathrm{C}_{8} \mathrm{H}_{8}\right)$. Each strip is read out by a wavelength shifting (WLS) fiber, Kuraray Y11(200)MS attached to a 64-channel multi-anode photomultiplier tube (MA-PMT), Hamamatsu H8804 as shown in Figure 3.5. The hit finding efficiencies evaluated with cosmic ray data are $99.8 \%$ and $99.9 \%$ for the vertical and horizontal planes, respectively. The track finding efficiency for single tracks of $10 \mathrm{~cm}$ or longer is more than $99 \%$. The radiation length of polystyrene (scintillator material) is approximately about $43 \mathrm{~cm}$. Table 3.1 summarizes specifications of the SciBar detector. The details of the readout electronics, the gain monitoring system and the energy calibration can be found elsewhere [76, 77].

\subsubsection{Electromagnetic Calorimeter (EC)}

The EC is a "spaghetti" type electromagnetic calorimeter, installed downstream of SciBar, and is designed to measure the electron neutrino contamination in the beam and identify photons from $\pi^{0}$ decay. The calorimeter modules were originally built for the CHORUS experiment at CERN [78] and later used in HARP and then in K2K.

The calorimeter consists of modules of dimensions $262 \times 8.4 \times 4.2 \mathrm{~cm}^{3}$. The modules construct one vertical and one horizontal plane, and each plane has 32 modules. An active area of $2.7 \times$ $2.6 \mathrm{~m}^{2}$ is covered by the planes. The EC has a thickness of 11 radiation lengths along the beam direction.

Each module is made of a stack of 21 lead sheets and 740 scintillating fibers. The $1 \mathrm{~mm}$ diameter scintillating fibers, Kuraray SCSF81, are embedded in the grooves on $1.9 \mathrm{~mm}$ thick lead sheets. The stack is held together by a welded steel case. At each end of the module, fibers are grouped into two bundles, and each bundle is connected to a Plexiglas light guide. The light guide is attached to 1 inch PMT, Hamamatsu R1335/SM, with a special green-extended photo-cathode. 


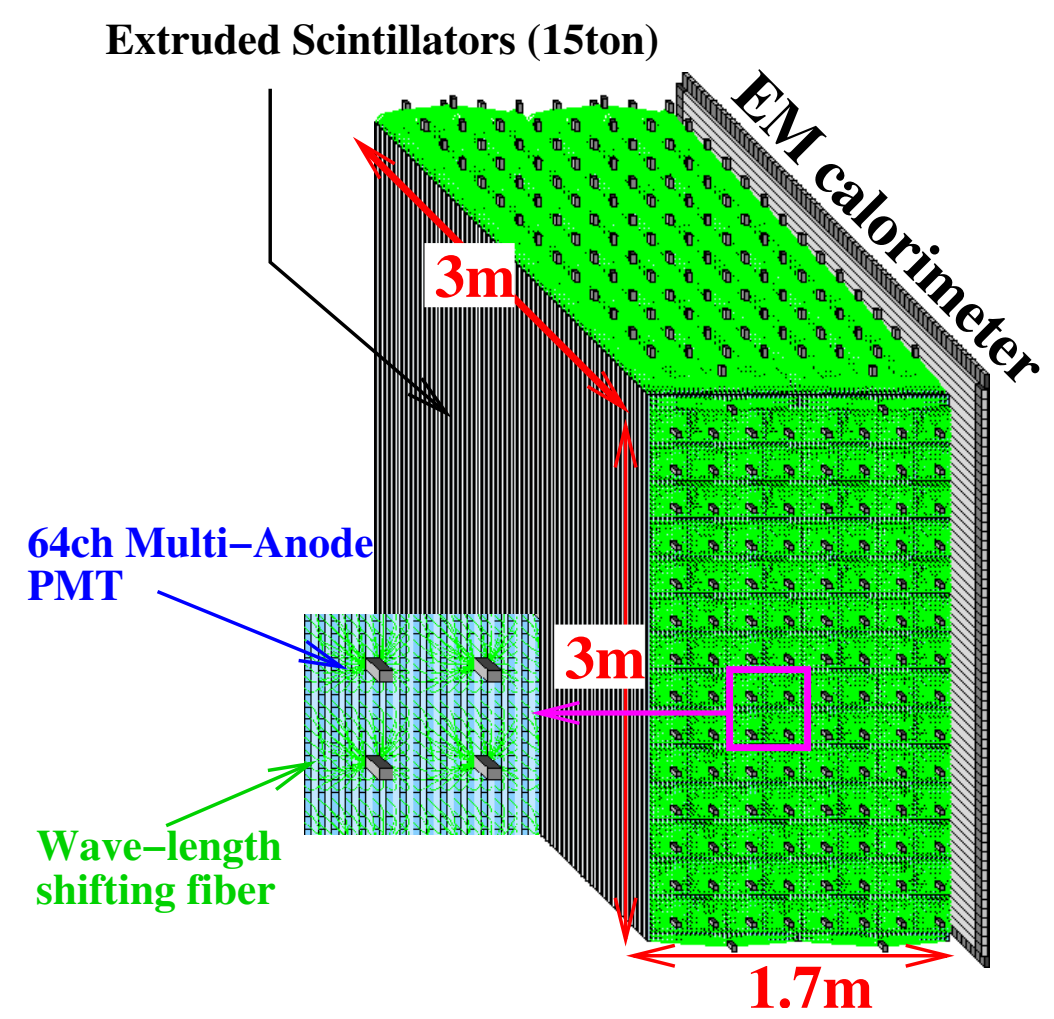

Figure 3.4: Schematic drawing of SciBar.

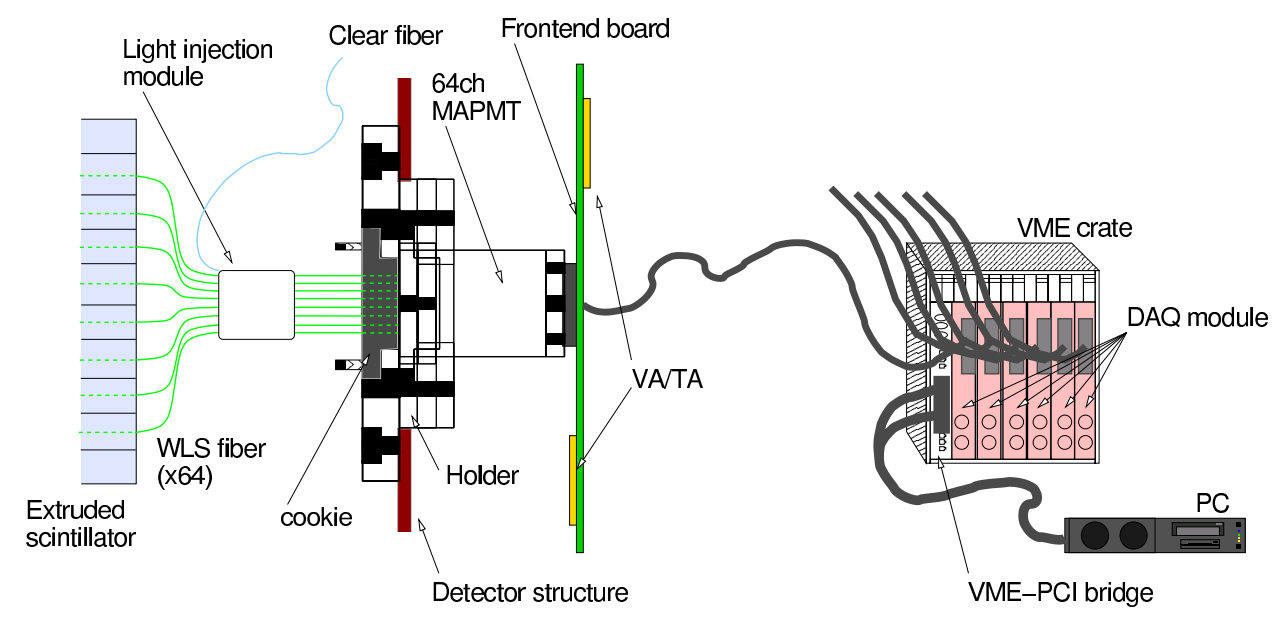

Figure 3.5: Schematic drawing of the SciBar readout system. 
Table 3.1: Specifications of the SciBar detector

\begin{tabular}{|c|c|}
\hline \multicolumn{2}{|r|}{ Structure } \\
\hline Dimensions & $3 \mathrm{~m} \times 3 \mathrm{~m} \times 1.7 \mathrm{~m}$ \\
\hline Weight & 15 tons \\
\hline Number of channels & 14,336 \\
\hline \multicolumn{2}{|r|}{ Scintillator } \\
\hline Material & Polystyrene, $\mathrm{PPO}(1 \%), \mathrm{POPOP}(0.03 \%)$ \\
\hline Emission peak wavelength & $420 \mathrm{~nm}$ \\
\hline Reflector material & $\mathrm{TiO}_{2}(15 \%)$ infused in polystyrene \\
\hline Dimensions & $1.3 \mathrm{~cm} \times 2.5 \mathrm{~cm} \times 300 \mathrm{~cm}$ \\
\hline Density & $1.021 \mathrm{~g} / \mathrm{cm}^{3}$ \\
\hline \multicolumn{2}{|r|}{ WLS fiber } \\
\hline Type & Kuraray Y11(200)MS, multi-clad \\
\hline Material & polystyrene(core), acrylic(inner), polyfluor(outer) \\
\hline Refractive index & 1.56(core), 1.49(inner), 1.42(outer) \\
\hline Absorption peak wavelength & $430 \mathrm{~nm}$ \\
\hline Emission peak wavelength & $476 \mathrm{~nm}$ \\
\hline Diameter & $1.5 \mathrm{~mm}$ \\
\hline Attenuation length & $350 \mathrm{~cm}$ (typical) \\
\hline \multicolumn{2}{|r|}{ MA-PMT } \\
\hline Model & Hamamatsu H8804 \\
\hline Anode & $8 \times 8$ pixels (pixel size: $2 \times 2 \mathrm{~mm}^{2}$ ) \\
\hline Cathode & Bialkali (Sb-K-Cs) \\
\hline Sensitive wavelength & 300-650 nm (peak: $420 \mathrm{~nm})$ \\
\hline Quantum efficiency & $12 \%$ at $\lambda=500 \mathrm{~nm}$ \\
\hline Dynode & Metal channel structure, 12 stages \\
\hline Gain & typical $6 \times 10^{5}$ at $800 \mathrm{~V}$ \\
\hline Response linearity & $\begin{array}{l}\text { within } 10 \% \text { up to } 200 \text { photo-electrons } \\
\text { with the gain of } 6 \times 10^{5}\end{array}$ \\
\hline Crosstalk & $3.15 \%($ adjacent pixel) \\
\hline \multicolumn{2}{|r|}{ Readout electronics } \\
\hline Number of ADC channels & 14,336 \\
\hline ADC pedestal width & less than 0.3 photo-electron \\
\hline $\mathrm{ADC}$ response linearity & $\begin{array}{l}\text { within } 5 \% \text { up to } 300 \text { photo-electrons } \\
\text { with the gain of } 5 \times 10^{5}\end{array}$ \\
\hline Number of TDC channels & 448 \\
\hline TDC resolution & $0.78 \mathrm{nsec}$ \\
\hline TDC full range & $50 \mu \mathrm{sec}$ \\
\hline
\end{tabular}


A typical gain is $2 \times 10^{6}$ at the operation voltage of $1600 \mathrm{~V}$. In total, 256 PMTs are used in the EC. The attenuation length of the fiber is measured to be approximately $400 \mathrm{~cm}$ by using cosmic-ray muons. The readout system consists of eight 32-channel 12-bit QDC (Charge-to-Digital Converter) modules, CAEN V792. One VME-6U crate is used for QDC readout. The energy resolution for electrons was measured to be $14 \% / \sqrt{E(\mathrm{GeV})}$ in a test beam [78].

\subsubsection{Muon Range Detector (MRD)}

The MRD is installed downstream of the EC and is designed to measure the momentum of muons produced by charged-current neutrino interactions up to $1.2 \mathrm{GeV} / c$ using the range measurement. The MRD was constructed for SciBooNE at FNAL, primarily by using out of parts recycled from past FNAL experiments.

Figure 3.6 show the schematic drawing of the MRD. The MRD consists of 12 iron plates and 13 alternating horizontal and vertical scintillator planes. Each iron plate is 2 inch thick, and covers an area of $274 \times 305 \mathrm{~cm}^{2}$. The total mass of absorber material is approximately 48 tons. The density of a spare iron plate was measured at several positions of the plate, to be $7.841 \pm 0.002 \mathrm{~g} / \mathrm{cm}^{3}$. The thickness of each plate was also measured prior to the experiment, with an accuracy of $1 \%$. The iron plates are sandwiched between scintillator planes. Each scintillator plane consists of $20 \mathrm{~cm}$ wide, $6 \mathrm{~mm}$ thick scintillator paddles, as shown in Fig. 3.7. Each vertical scintillator plane is comprised of $138 \mathrm{~cm}$ long paddles, arranged in a $2 \times 15$ array to have an active area of $276 \times 300 \mathrm{~cm}^{2}$. On the other hand, each horizontal scintillator plane consists of $155 \mathrm{~cm}$ long paddles, arranged in a $13 \times 2$ array to have an active area of $260 \times 310 \mathrm{~cm}^{2}$. In total, 362 paddles are used in the MRD. The iron plates and scintillator paddles are recycled from the FNAL E605 experiment [79].

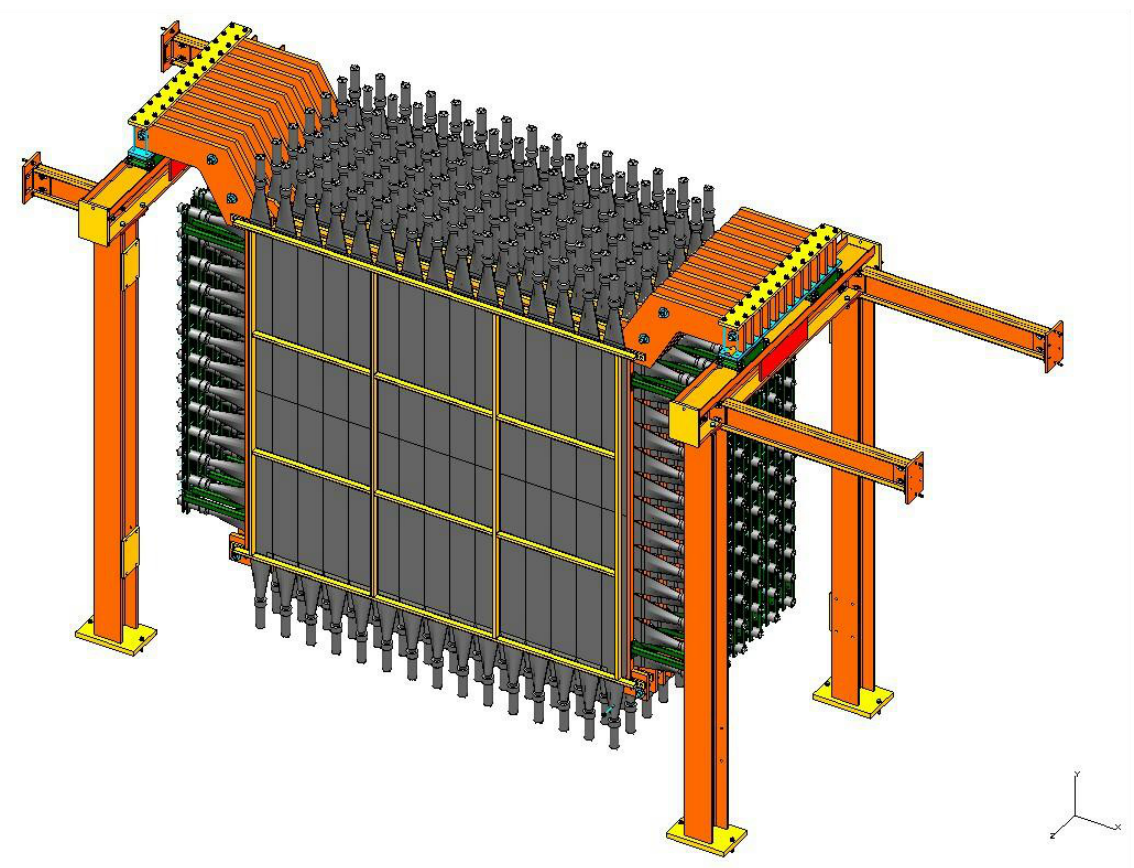

Figure 3.6: Schematic drawing of the MRD detector

Photon detectors The scintillator paddles are read out by five types of 2 inch PMTs; the vertical planes consist of Hamamatsu 2154-05 and RCA 6342A PMTs, the horizontal planes consist of EMI 9954KB, EMI 9839b and 9939b PMTs. All PMTs used for horizontal modules has 14 stage dynodes, while those used for vertical modules has 10 stage dynodes. We use these shorter PMTs for vertical modules because of the space limitations. Hence, the PMTs used for the vertical planes, i.e. Hamamatsu 2154-05 and RCA 6342, have relatively low gain and efficiency compared to that used 


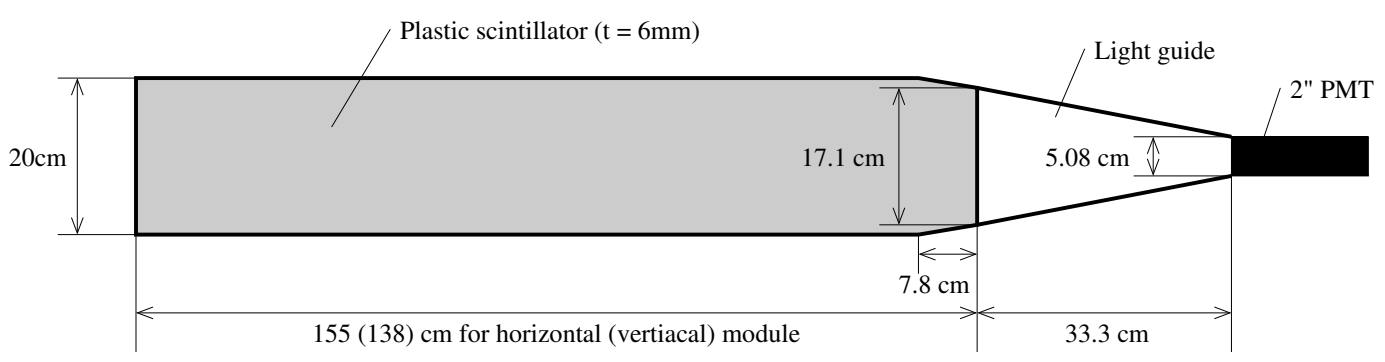

Figure 3.7: Schematic drawing of a scintillator paddle for the MRD

for horizontal planes. To ensure the enough efficiencies for vertical modules, the signal from the vertical modules are amplified by the factor of 10 using LeCroy 612 amplifiers.

Readout electronics We record charge and timing information from each PMT. The readout electronics system consists of LeCroy 4300B ADCs and LeCroy 3377 TDCs. We use 13 TDCs and 26 ADCs housed in three CAMAC crates. The timing resolution and full range are $0.5 \mathrm{~ns}$ and $32 \mu \mathrm{sec}$, respectively. The energy threshold for TDC hits is approximately $250 \mathrm{keV}$ which corresponds to $20 \%$ of the signal of a minimum ionizing particle. The single noise rate is typically $100 \mathrm{~Hz}$ except for RCA 6342A PMTs, which has high noise rate up to $10^{4} \mathrm{~Hz}$. Although some of RCA PMTs have high noise rate, these noise are removed by requiring coincidence with hits in other MRD layers or hits in the SciBar detector.

Table 3.2: Specifications of the MRD detector.

\begin{tabular}{ll}
\hline \hline & Iron plate \\
\hline Number of plates & 12 \\
Dimensions & $274 \times 305 \mathrm{~cm}^{2}, 2$ inch thickness \\
Density & $7.841 \mathrm{~g} / \mathrm{cm}^{3}$ \\
\hline & Scintillator plane \\
\hline Number of planes & 13 \\
Segmentation & $2 \times 15$ (vertical), $13 \times 2$ (horizontal) \\
Dimensions of a counter & thickness: $6 \mathrm{~mm}$, width: $20 \mathrm{~cm}$ \\
& length: $138 \mathrm{~cm}$ (vertical), $155 \mathrm{~cm}$ (horizontal) \\
\hline & \multicolumn{1}{c}{ PMT } \\
\hline Model & EMI $9954 \mathrm{~KB}, 9839 \mathrm{~b}$ and $9939 \mathrm{~b}$ (horizontal) \\
& $\sim 1 \times 10^{6}$ at $1200 \mathrm{~V}$ (vertical) \\
Gain & $\sim 1 \times 10^{7}$ at $2000 \mathrm{~V}$ (horizontal) \\
& Readout electronics \\
& 362 \\
Number of channels & LeCroy $4300 \mathrm{~B}$ (ADC), Lecroy 3377 (TDC) \\
Model & $0.5 \mathrm{nsec}$ \\
TDC resolution & $32 \mu \mathrm{sec}$ \\
TDC full range &
\end{tabular}

Performance monitoring with cosmic rays The MRD has a cosmic-ray trigger independent from SciBar and the EC. Figure 3.8 shows the schematic diagram of the cosmic ray trigger for the MRD. The triggers are generated using either horizontal or vertical layers. We require coincident hits of the most upstream and downstream layers to detect cosmic ray muons penetrate all MRD 
layers horizontally. Triggers generated by the vertical planes are used to test performance of the horizontal planes, and vice versa. Hit finding efficiency and the gain of PMTs were continuously monitored by using cosmic ray data taken between beam spills. Figure 3.9 shows the hit finding efficiency as a function of position for a typical scintillator plane. The average hit finding efficiency is measured to be $99 \%$. The main source of inefficiency comes from the gaps between counters which are typically $0.3 \mathrm{~cm}$.

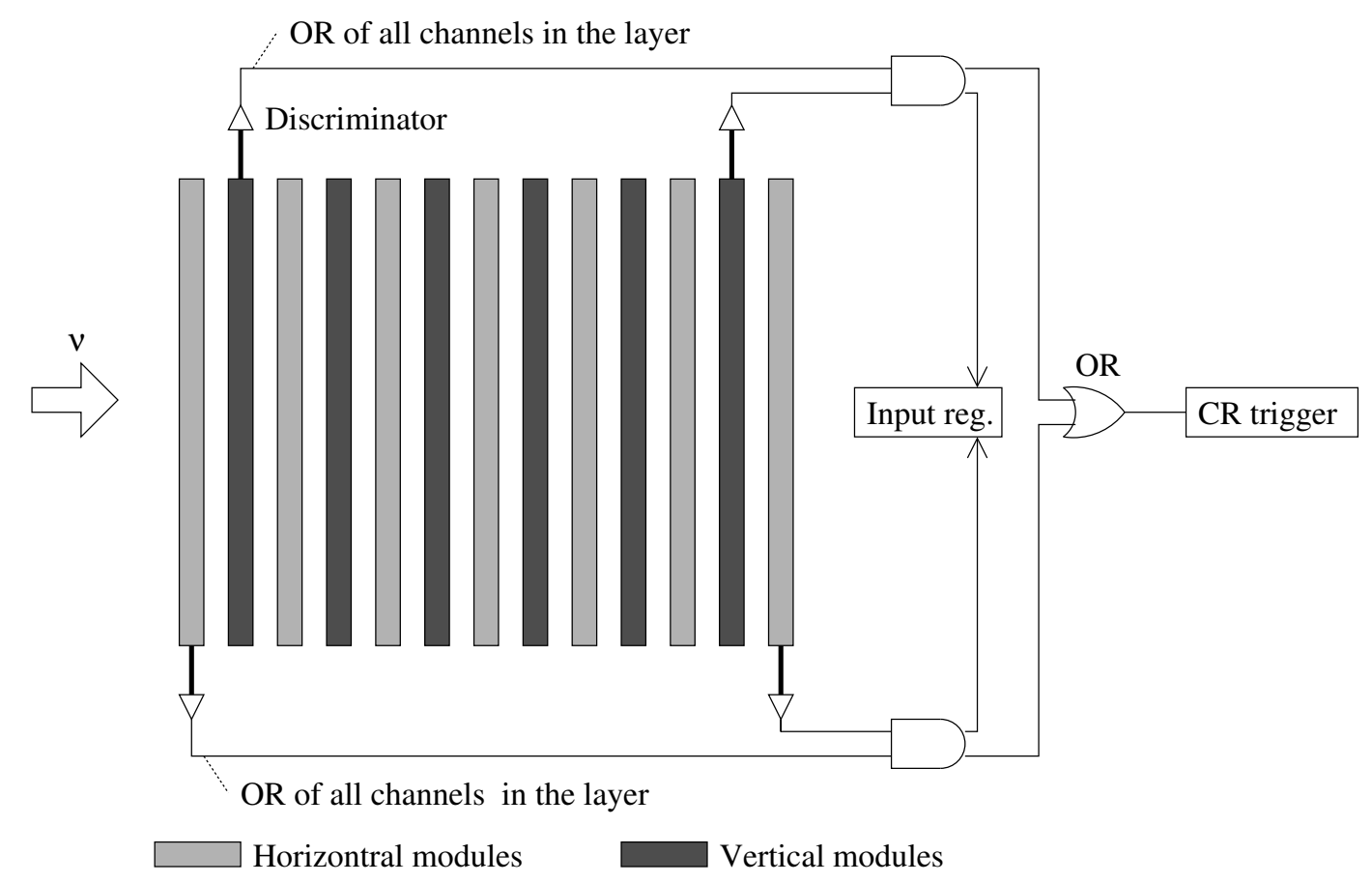

Figure 3.8: Schematic diagram of the MRD cosmic trigger. The trigger is generated using either horizontal or vertical layers, and the coincident hits of the most upstream and downstream layers are required. The input register is use to identify the generated trigger type.

\subsubsection{Trigger and data acquisition (DAQ) system}

The BNB recieves one train of proton beam pulses for each 2 sec cycle of the accelelation at the Booster. Each cycle contain typically $\sim 5$ proton pulses in a row at $15 \mathrm{~Hz}$. A beam trigger is generated by a fast timing singal sent by the extraction mangent on BNB. Then, we read all channels from all subdetectors for every beam triggers, regardless of the hit occupancy, to ensure unbiased beam data. The trigger timing is recorded using a Grobal Timing Positioning System (GPS), and is used to combine the beam data and the accelelerator information data.

Between each sequence of beam triggers, we take pedestal, LED (only for SciBar) and cosmic ray data, to monitor the detector performances. We have independent cosmic triggers for SciBar/EC and for MRD, which both collected 20 cosmic ray triggers in a cycle.

More detailed descriptions of our trigger and DAQ systems can be found in Ref. [77].

\subsubsection{MiniBooNE detector}

The MiniBooNE detector is located $440 \mathrm{~m}$ downstream from the SciBooNE detector. The detector is a $12.2 \mathrm{~m}$ diameter spherical tank filled with 800 tons of mineral oil $\left(\mathrm{CH}_{2}\right)$. Figure 3.10 is a schematic drawing of the MiniBooNE detector.

The detector is designed to measure Cherenkov lights from particles produced by neutrino interactions with the mineral oil. The detection medium is pure Marcol 7 mineral oil; the density 


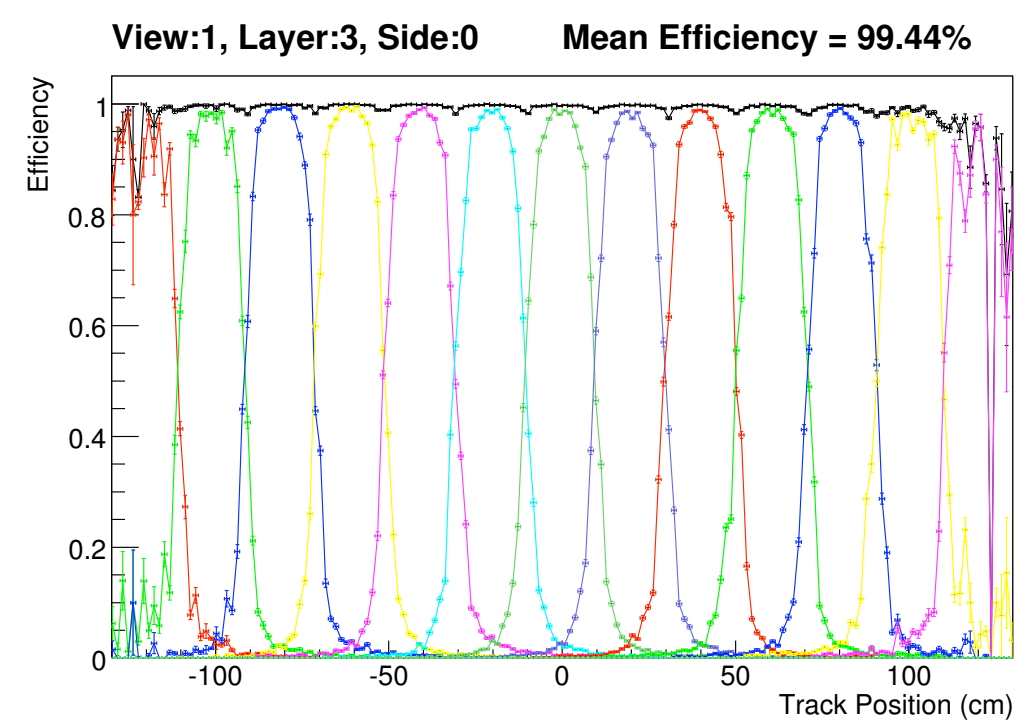

Figure 3.9: Hit finding efficiency as a function of position for a typical scintillator plane. Both individual counter efficiencies (colored points) and the summed efficiency (black points) are shown.
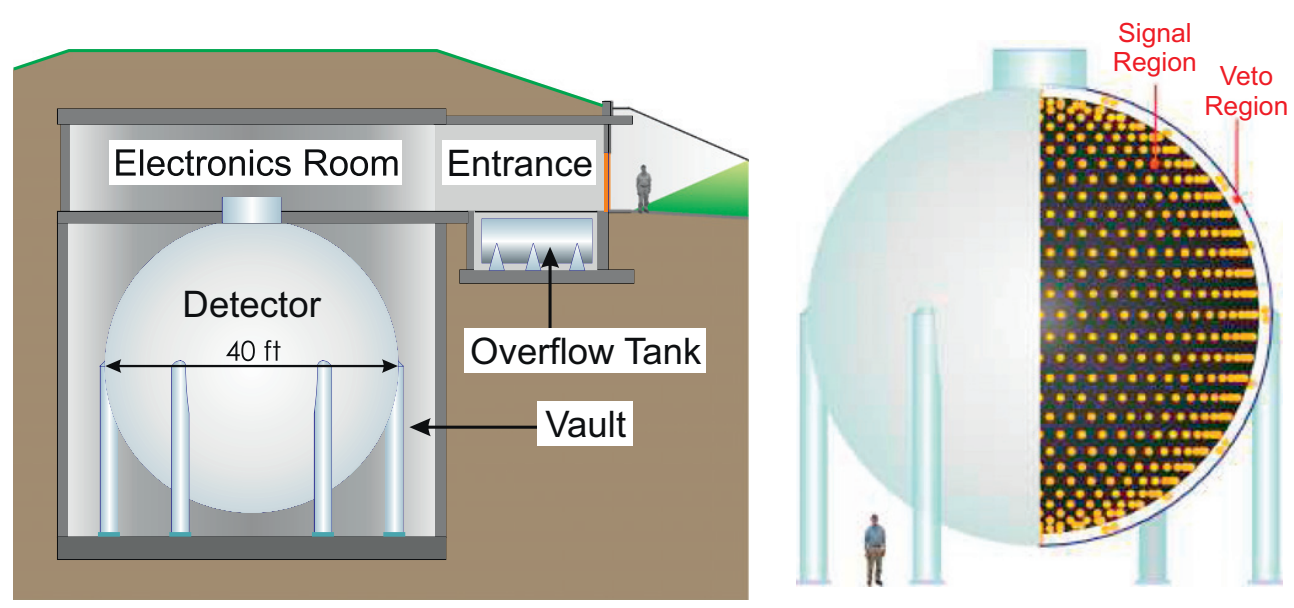

Figure 3.10: Schematic drawing of the MiniBooNE detector. The MiniBooNE detector enclosure (left) and a cut-away drawing (right) of the detector showing the distribution of PMTs in the signal and veto regions. This picture is taken from Ref. [80]. 
$(\rho)$ and the refractive index $(n)$ of the oil are measured to be $\rho=0.845 \pm 0.001 \mathrm{~g} / \mathrm{cm}^{3}$ and $n=1.47$, respectively.

Details of detector structure, simulation, and error analysis are available in Refs. [80, 81].

\subsection{Data sets}

Table 3.3 summarizes the history of the BNB operation and recorded POT at SciBooNE and MiniBooNE detectors. The BNB started operation with neutrino mode in Sep. 2002 for MiniBooNE, and was running with neutrino mode from Sep. 2002 to Dec. 2005, and from Oct. 2007 to Apr. 2008. The first anti-neutrino run was conducted between Jan. 2006 and Aug. 2007, and the second run started in Apr. 2008, which is still providing anti-neutrino beam for MiniBooNE to the present. The SciBooNE experiment started in Jun. 2007, during the first anti-neutrino operation of the BNB, and finished data taking in Aug. 2008. Figure 3.11 show the history of the POT delivered to the BNB, for the entire running period since September 2002, and for the SciBooNE-MiniBooNE joint run period.

Table 3.3: Summary of corrected POT at SciBooNE and MiniBooNE detectors.

\begin{tabular}{llll}
\hline \hline Period & BNB Mode & SciBooNE POT & MiniBooNE POT \\
\hline Sep. 2002 - Dec. 2005 & Neutrino & - & $5.58 \times 10^{20}$ \\
Jan. 2006 - Aug. 2007 & Anti-neutrino & $0.52 \times 10^{20}$ (from Jun. 2007) & $1.71 \times 10^{20}$ \\
Oct. 2007 - Apr. 2008 & Neutrino & $0.99 \times 10^{20}$ & $0.83 \times 10^{20}$ \\
Apr. 2008 - present & Anti-neutrino & $1.01 \times 10^{20}$ (until Aug. 2008) & ongoing \\
\hline \hline
\end{tabular}

The analysis presented here uses the full neutrino mode data sets collected at SciBooNE and MiniBooNE. The amount of SciBooNE data collected in the neutrino mode is $0.99 \times 10^{20}$ protons on target (POT) taken between October 2007 and April 2008.

The MiniBooNE experiment has been taking beam data since 2002, including the SciBooNE and MiniBooNE joint-run period. The collected number of POT after data quality cut in the neutrino mode is $5.579 \times 10^{20}$ in addition to the data from the joint-run period, which is $0.83 \times 10^{20}$ POT. The difference of collected POT during the SciBooNE and MiniBooNE joint run period is due to different live-times between the detectors and a known inefficiency in the MiniBooNE reprocessing [82]. 

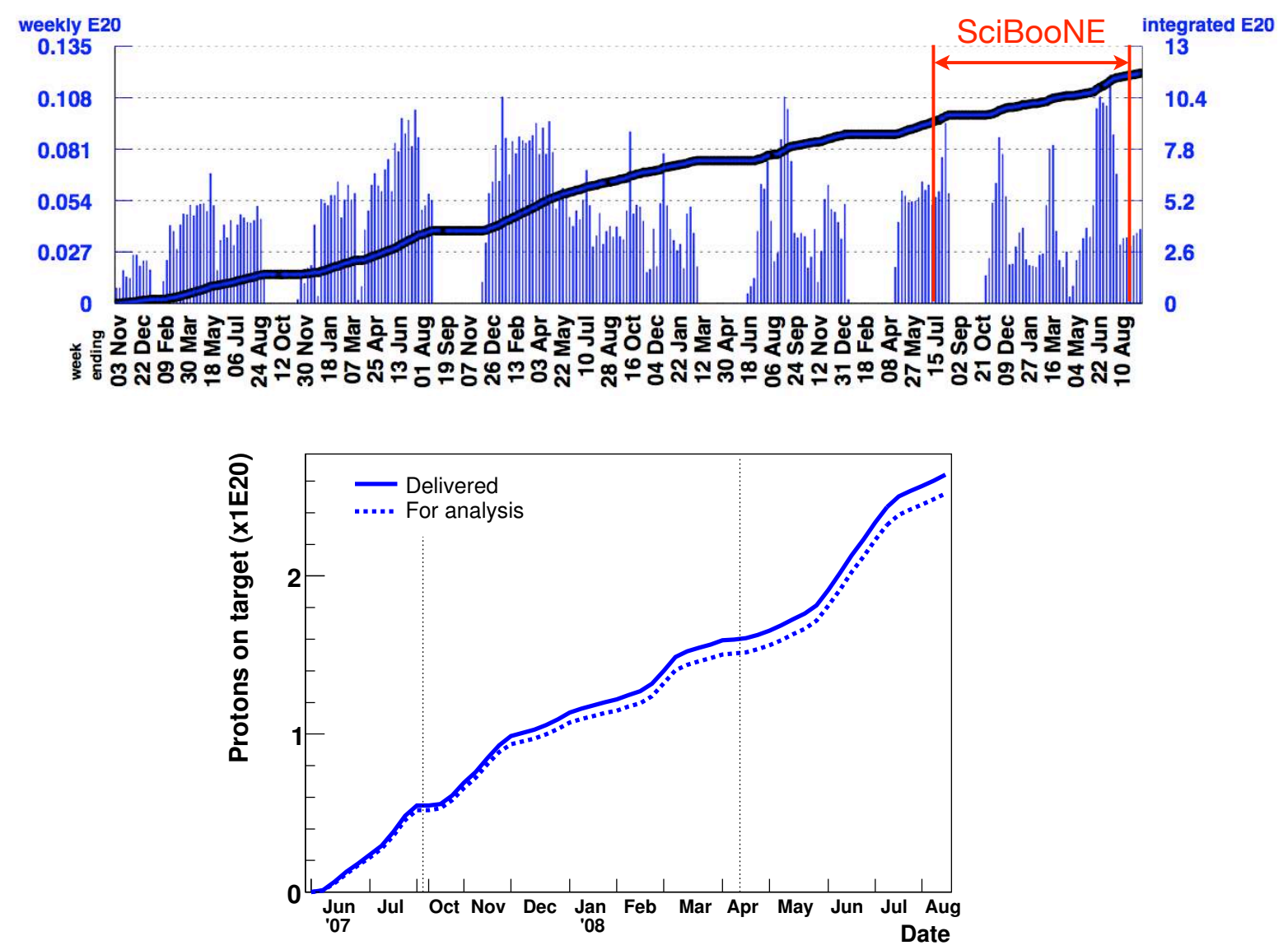

Figure 3.11: Top: Protons delivered to the BNB, since the beamline commissioning in September 2002. The SciBooNE-MiniBooNE joint run period is also indicated. Bottom: Protons delivered during SciBooNE data taking period. 


\section{Chapter 4}

\section{Monte carlo simulation}

We simulate neutrino events at SciBooNE with the following three steps:

- Neutrino flux simulation

- Neutrino interaction simulation

- Detector response simulation

We use a common framework to predict Neutrino fluxes at SciBooNE and MiniBooNE, and then the output of the flux simulations are passed to Neutrino interaction simulation programs. NEUT [71] and NUANCE [73] libraries are used to simulate neutrino interactions at SciBooNE, while only NUANCE is used for MiniBooNE. Finally, information of the final state particles emerging from neutrino interactions are passed to the detector response simulations.

In this section, we describe these simulations in detail.

\subsection{Neutrino flux simulation}

Predictions for the BNB neutrino flux illuminating the SciBooNE and MiniBooNE are made using a program developed by the MiniBooNE group [43], which consists of the following two steps:

1. Simulation of meson production and tracking in the beamline with a GEANT4 [83] program, and

2. Simulation of the meson decay and tracking of produced neutrinos with a FORTRAN-based program.

In the step (1), we simulate meson productions from the $8.9 \mathrm{GeV} / \mathrm{c}$ proton-Be interactions, and trace the meson trajectories in the horn magnetic field and the decay volume, until they decay into neutrinos. In the step (2), we simulate those mesons decays multiple times to boost the MC statistics. Then, the information (kinematics and position) of neutrinos and their parent mesons are saved, if the neutrino trajectory hit SciBooNE or MiniBooNE detectors.

In the following part, we describe these neutrino flux simulation in more detail.

\subsubsection{Simulation of meson productions}

In the simulation, the geometry, location, and material compositions of the component of the BNB are implemented, including the Be target, the shape of the focusing horn and the magnetic field produced by the horn.

The production cross sections of $\mathrm{p}, \mathrm{n}, \pi^{+}, \pi^{-}, K^{+}, K^{-}$and $K^{0}$ from $8.9 \mathrm{GeV} / \mathrm{c}$ proton-Be interactions are simulated by a custom model. 
For $\pi^{+}, \pi^{-}$, and $K^{0}$ productions, the Sanford-Wang parametrization [84] is used to calculate the double differential cross section of a given meson species:

$$
\frac{d^{2} \sigma}{d p d \Omega}=C_{1} p^{C_{2}}\left(1-\frac{p}{p_{B}-C_{9}}\right) \exp \left(-C_{3} \frac{p^{C_{4}}}{p_{B}^{C_{5}}}-C_{6} \theta\left(p-C_{7} p_{B} \cos ^{C_{8}} \theta\right)\right)
$$

where $p$ is the total momentum of the meson, $\theta$ is the angle of the meson with respect to the incident proton, $p_{B}$ is the momentum of the incident proton, and $C_{1}, \ldots, C_{9}$ are parameters determined by fitting to the experimental data.

For $\pi^{+}$and $\pi^{-}$production, which are most important processes for neutrino and anti-neutrino flux prediction, the parameters are obtained by fitting HARP [85] and BNL E910 [86] data. The Sanford-Wang parameters used in the simulation are summarized in Table 4.1 .

Table 4.1: Sanford-Wang parameters used in the simulation

\begin{tabular}{cccccccccc}
\hline \hline & $C_{1}$ & $C_{2}$ & $C_{3}$ & $C_{4}$ & $C_{5}$ & $C_{6}$ & $C_{7}$ & $C_{8}$ & $C_{9}$ \\
\hline$\pi^{+}$ & 220.7 & 1.080 & 1.000 & 1.978 & 1.32 & 5.572 & 0.0868 & 9.686 & 1 \\
$\pi^{-}$ & 213.7 & 0.9379 & 5.454 & 1.210 & 1.284 & 4.781 & 0.07338 & 8.329 & 1 \\
\hline \hline
\end{tabular}

For $K^{+}$production, since no published data exist for $K^{+}$production at the BNB primary proton beam energy, we employ the Feynman scaling hypothesis to relate $K^{+}$production measurements at different proton beam energies to the expected production at the BNB proton beam energy [43]. In this hypothesis, the Feynman scaling variable $x_{F} \equiv p_{\|}^{\mathrm{cm}} / p_{\|}^{\max , \mathrm{cm}}$ is used, where $p_{\|}^{\mathrm{cm}}$ and $p_{\|}^{\text {max,cm }}$ is the parallel component of the momentum of the produced particle in the center-of-mass frame and the maximum value of $p_{\|}^{\mathrm{cm}}$ for the given reaction, respectively. The double differential cross section using $x_{F}$ is expressed as:

$$
\frac{d^{2} \sigma}{d p d \Omega}=\frac{p^{2}}{E^{2}} C_{1}\left(1-\left|x_{F}\right|\right) \exp \left(-C_{2} p_{T}-C_{3}\left|x_{F}\right|^{C_{4}}-C_{5} p_{T}^{2}-C_{7}\left|p_{T} \times x_{F}\right|^{C_{6}}\right)
$$

where $p, p_{T}$ and $E$ are the momentum, the transverse component of the momentum and energy of $K^{+}$, respectively. For $K^{-}$production, the MARS hadronic interaction package [87] is used to determine the absolute double differential cross section.

Table 4.2: Feynman scaling parameters used in the simulation

\begin{tabular}{cccccccc}
\hline \hline & $C_{1}$ & $C_{2}$ & $C_{3}$ & $C_{4}$ & $C_{5}$ & $C_{6}$ & $C_{7}$ \\
\hline$K^{+}$ & 11.70 & 0.88 & 4.77 & 1.51 & 2.21 & 2.17 & 1.51 \\
\hline \hline
\end{tabular}

The most comprehensive description of the flux prediction can be found in Ref. [43].

\subsubsection{Simulation of meson decays}

The FORTRAN-based Monte Carlo code uses the output of the meson productions simulation as input, and generates the neutrino kinematics distributions from meson and muon decays. Current best knowledge of meson and muon decay branching fractions, and decay form factors in three-body semi-leptonic decays are used [76]. Polarization effects in muon decays are also accounted for.

Neutrinos produced by the meson decays are extrapolated along straight lines toward the SciBooNE and MiniBooNE detectors. All neutrinos whose ray traces cross the detector volumes are used for the SciBooNE and MiniBooNE flux predictions. 
We simulate these decay processes multiple times ("re-decay") for each meson to reduce the statistical uncertainties of the MC predictions. In the case of SciBooNE, we re-decay 10 times for each meson, while it is 1000 for MiniBooNE. We set smaller number of re-decay for SciBooNE because the acceptance of the SciBooNE detector is much larger than MiniBooNE, and found that we do not gain statistical significance by increasing the number of re-decay to be more than 10 .

\subsubsection{Neutrino beam flux prediction}

The neutrino flux prediction at the SciBooNE and MiniBooNE detector locations and as a functions of neutrino energy are shown in Figure 4.1. We can see that neutrino spectrum at SciBooNE and MiniBooNE are very similar, except for the flux normalization difference. For the neutrino running mode, a total neutrino flux per proton on target of $2.2 \times 10^{-8} \mathrm{~cm}^{-2}$ and $5.2 \times 10^{-10} \mathrm{~cm}^{-2}$ are expected at the SciBooNE and MiniBooNE detectors, respectively. The flux is dominated by muon neutrinos (93\%). The $\nu_{\mu}$ energy spectrum peaks at $\sim 0.6 \mathrm{GeV}$, and extends up to $2-3 \mathrm{GeV}$. The mean energy of the $\nu_{\mu}$ flux is expected to be $0.76 \mathrm{GeV}$ for SciBooNE and $0.79 \mathrm{GeV}$ for MiniBooNE.
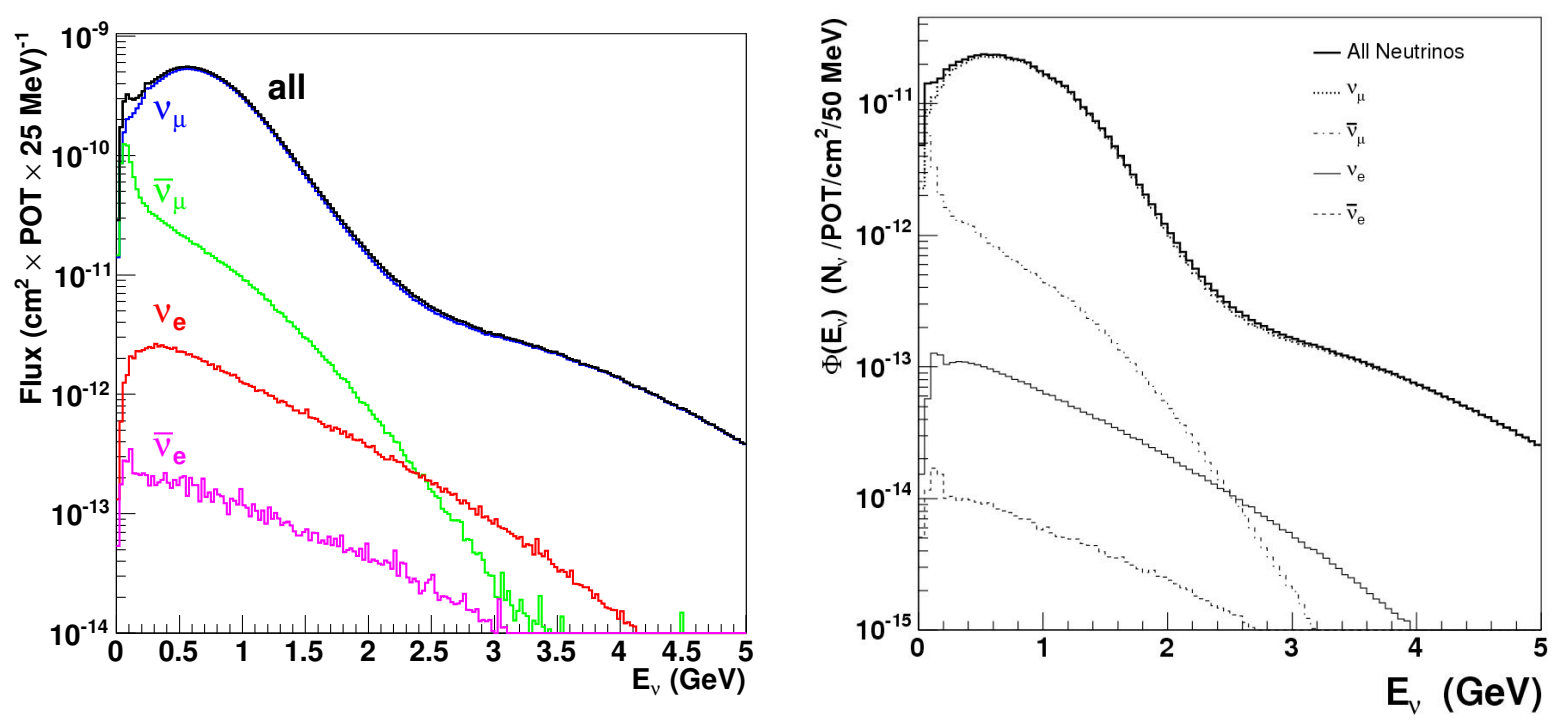

Figure 4.1: Neutrino flux prediction at the SciBooNE detector (left panel) and the MiniBooNE detector (right panel) as a function of neutrino energy $E_{\nu}$, normalized per unit area, proton on target (POT) and neutrino energy bin width. The total flux and contributions from individual neutrino flavors are shown.

\subsubsection{Systematic uncertainty of the flux prediction}

Figure 4.2 shows the $\nu_{\mu}$ flux from $\pi^{+}$and $K^{+}$decays, and their fractional uncertainties.

The largest error on the predicted neutrino flux results from the uncertainty of pion production in the initial p-Be process in the target. The simulation predicts that $96.7 \%$ of muon neutrinos in the BNB are produced via $\pi^{+}$decay. The uncertainty in $\pi^{+}$production is determined from spline fits to the HARP $\pi^{+}$double differential cross section data [43]. To estimate the size of error, we first produce a 1000 HARP fake-results randomly drown from the HARP measurement errors. Then, we fit each fake-result with spline functions to interpolate and extrapolate the data points. Figure 4.3 shows the results of spline interpolation and Sanford-Wang fit for the double differential $\pi^{+}$production cross section $\left(d^{2} \sigma /\left(d p_{\pi} d \Omega_{\pi}\right)\right)$. The difference of spline functions obtained for HARP central value and fake-results are accounted as systematic uncertainty.

The resulting $\pi^{+}$production uncertainty is $\approx 5 \%$ at the peak of the flux distribution and increases significantly at high and low neutrino energies. 

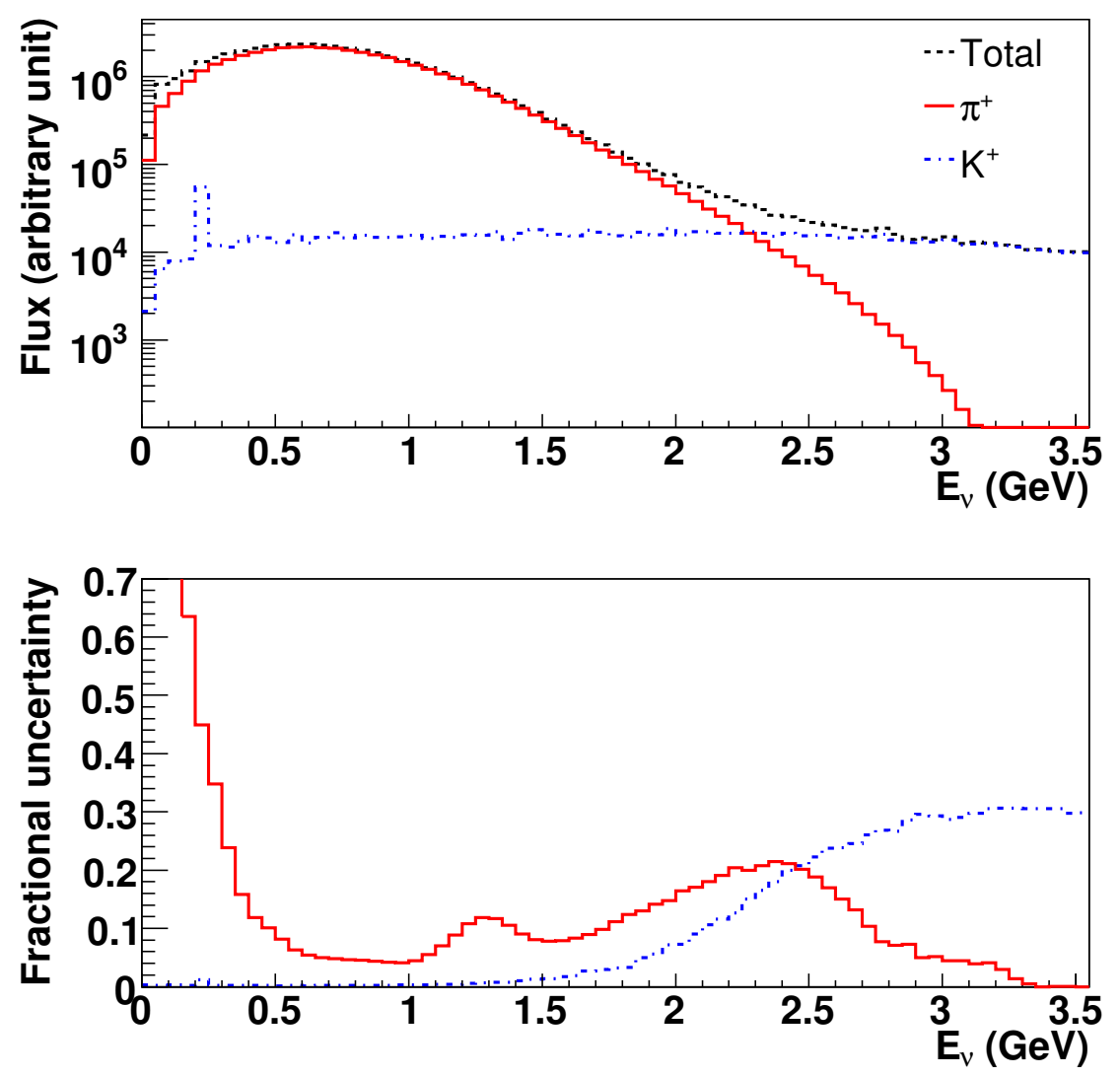

Figure 4.2: (Top) $\nu_{\mu}$ flux prediction at the SciBooNE detector as a function of neutrino energy $E_{\nu}$. The total flux and contributions from $\pi^{+}$and $K^{+}$decays are shown. (Bottom) Fractional uncertainty of the $\nu_{\mu}$ flux prediction due to $\pi^{+}$and $K^{+}$production from the p-Be interaction. Additional uncertainties from POT, hadron interactions in the target, and the horn magnetic field are not shown. 

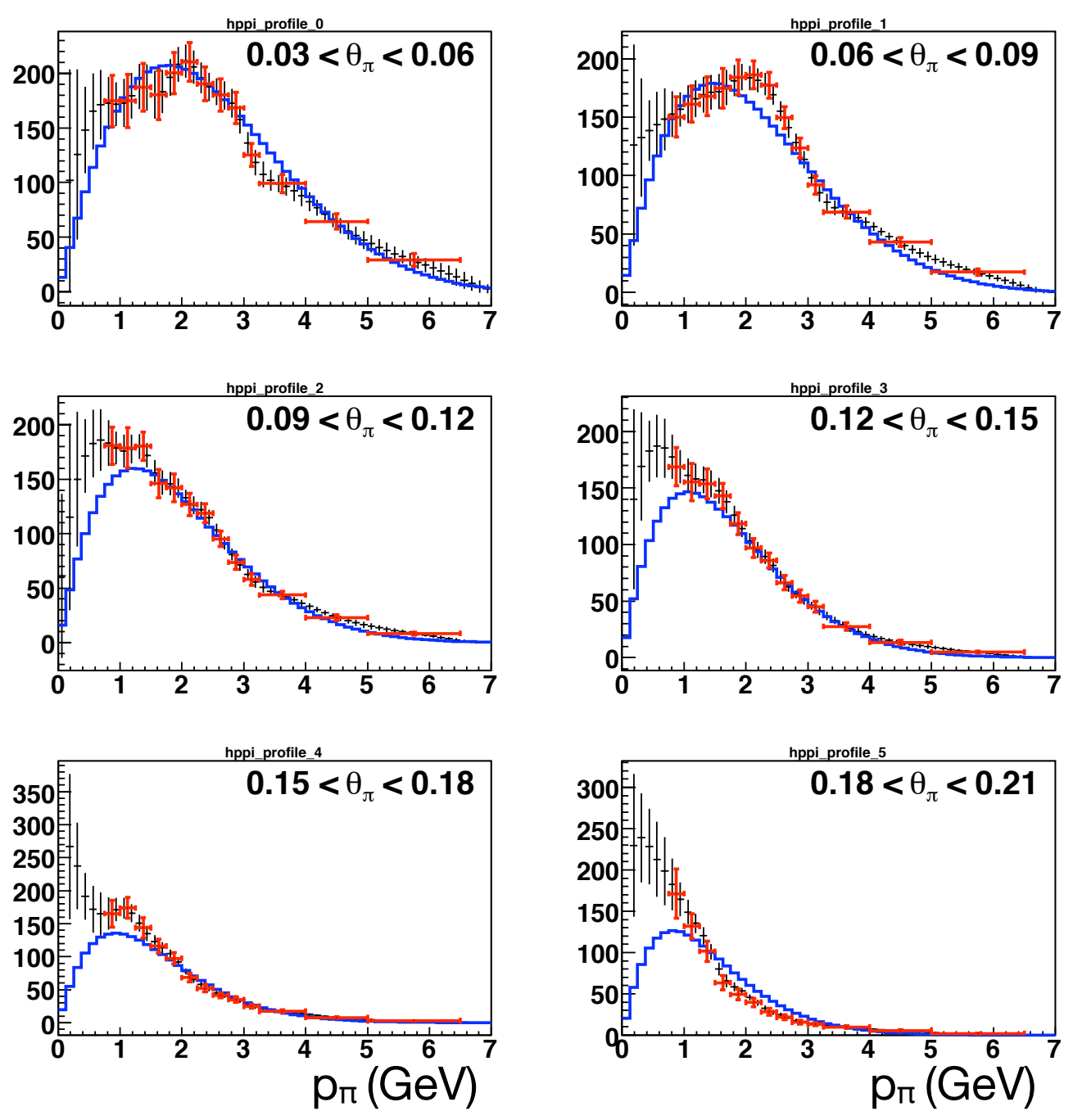

Figure 4.3: Double differential $\pi^{+}$production cross section $\left(d^{2} \sigma /\left(d p_{\pi} d \Omega_{\pi}\right)\right)$ from $8.9 \mathrm{GeV} / \mathrm{c} \mathrm{p}-\mathrm{Be}$ interaction. The red points show the HARP data, and the blue curve shows the best fit to the data with the Sanford-Wang function, which used to produce the MC central value. The black points show the profile of the spline curves produced by the HARP data points and their errors. 
The flux from $K^{+}$decay is dominant for $E_{\nu}>2.3 \mathrm{GeV}$. For the $K^{+}$production uncertainty, the errors of the Feynman scaling parameters obtained from these measurements are accounted for in the systematic error. The size of the errors of the Feynman scaling parameters and their correlations can be found in Ref. [43].

Other major contributions to the flux error include uncertainties on hadron interactions in the target and simulation of the the horn magnetic field, which both contribute to shape and normalization uncertainties, as well as the measurement of the number of POT, which is a pure normalization uncertainty. All flux errors are modeled through variations in the simulation and result in a total error of $\approx 7 \%$ at the peak of the flux at SciBooNE. The flux errors at MiniBooNE detector can be found elsewhere [43, 66], and are very similar to those for SciBooNE.

\subsection{Neutrino interaction simulation}

\subsubsection{Overview}

We simulate neutrino interactions with carbon and hydrogen in the SciBar detector with the NEUT and NUANCE program libraries. For simulating neutrino interactions in the MiniBooNE detector, we only use NUANCE library.

Then, for the studies of neutrino Charged Current interaction at SciBooNE, we produced separate Monte Carlo (MC) samples with NEUT and NUANCE and compared the two to the SciBooNE neutrino data, while only the NUANCE based MC is used for the oscillation studies with MiniBooNE.

The nuclear targets handled in NEUT are protons, carbon, oxygen, and iron. The energy of neutrinos handled by the simulation ranges from $100 \mathrm{MeV}$ to $100 \mathrm{TeV}$. The types of neutrino interactions simulated in both $\mathrm{NC}$ and $\mathrm{CC}$ are : elastic and quasi-elastic scattering $\left(\nu N \rightarrow \ell N^{\prime}\right)$, single meson production $\left(\nu N \rightarrow \ell N^{\prime} m\right)$, single gamma production $\left(\nu N \rightarrow \ell N^{\prime} \gamma\right)$, coherent $\pi$ production $\left(\nu^{12} \mathrm{C}\left(\right.\right.$ or $\left.^{56} \mathrm{Fe}\right) \rightarrow \ell \pi{ }^{12} \mathrm{C}\left(\right.$ or $\left.\left.^{56} \mathrm{Fe}\right)\right)$, and deep inelastic scattering $\left(\nu N \rightarrow \ell N^{\prime}\right.$ hadrons $)$, where $N$ and $N^{\prime}$ are the nucleons (proton or neutron), $\ell$ is the lepton (electron, muon or neutrino), and $m$ is the meson. In nuclei, interactions of the mesons and hadrons with the nuclear medium are simulated following the neutrino interactions.

The types and models of neutrino interactions in NUANCE are similar to those in NEUT but with different cross section parameter settings in some cases and a completely independent treatment of meson and hadron re-interactions in the nuclear medium.

In addition to the neutrino interactions inside SciBar, we also simulate interactions in the EC/MRD and the surrounding materials (the walls of the detector hall and soil) using NEUT. We assign a $20 \%$ normalization uncertainty to the interaction cross sections for both the EC/MRD and surrounding material relative to the predictions for SciBar.

\subsubsection{Neutrino interaction model}

\subsubsection{Quasi-elastic scattering}

The dominant interaction in the SciBooNE neutrino energy range is CC-QE scattering, which is implemented using the Smith and Moniz model [49]. The nucleons are treated as quasi-free particles and the Fermi motion of nucleons along with the Pauli exclusion principle is taken into account. The Fermi surface momentum $\left(p_{F}\right)$ for carbon is set to $217(220) \mathrm{MeV} / c$ and the nuclear potential $\left(E_{B}\right)$ is set to $25(34) \mathrm{MeV} / c$ in NEUT(NUANCE), as extracted from electron scattering data [88. The default binding energy in NUANCE is somewhat higher because it additionally accounts for neutrino vs. electron scattering differences [48]. The systematic errors for $p_{F}$ and $E_{B}$ are set to $\pm 30 \mathrm{MeV} / c$ and $\pm 9 \mathrm{MeV} / c$, respectively, for both NEUT and NUANCE.

For the vector form factor, NEUT uses a dipole form with a vector mass of $0.84 \mathrm{GeV} / \mathrm{c}^{2}$, while NUANCE uses the BBA-2003 form factor [89]. A dipole form is used for the axial form factor 
with an adjustable axial mass, $M_{A}^{Q E}$, for both NEUT and NUANCE. In NUANCE, an empirical Pauli-blocking parameter, $\kappa$, is introduced [66] to better describe the MiniBooNE quasi-elastic data at low momentum transfer. When $\kappa>1$, the phase space of nucleons susceptible to Pauli-blocking is increased and hence the cross section at low momentum transfer is suppressed.

The values of $M_{A}^{Q E}=1.21 \mathrm{GeV} / \mathrm{c}^{2}$ and $\kappa=1.000$ (i.e. no additional Pauli blocking adjustment) are used in NEUT, and $M_{A}^{Q E}=1.23 \mathrm{GeV} / \mathrm{c}^{2}$ and $\kappa=1.022$ are used in NUANCE [48. A systematic uncertainty of $\pm 0.22 \mathrm{GeV}$ is assigned to $M_{A}^{Q E}$ to span the difference between the value used and the global fit from previous measurements [90]. The difference between $\kappa=1.000$ and $\kappa=1.022$ is also assigned as systematic uncertainty.

The same Fermi momentum distribution, nuclear potential are used in all other neutrino-nucleus interactions except for coherent $\pi$ production.

\subsubsection{Meson production via baryon resonances}

The second most frequent interaction in SciBooNE is the resonant production of single pion, kaon, and eta mesons as described by the model of Rein and Sehgal (RS) [91].

The RS model assumes an intermediate baryon resonance, $N^{*}$, in the reaction of $\nu N \rightarrow$ $\ell N^{*}, N^{*} \rightarrow N^{\prime} m$. All intermediate baryon resonances with mass less than $2 \mathrm{GeV} / c^{2}$ are included. Baryon resonances with mass greater than $2 \mathrm{GeV} / c^{2}$ are simulated as deep inelastic scattering. $\Delta$ re-interactions $(\Delta N \rightarrow N N)$ which do not lead to a mesonic final state are also simulated. This re-interaction probability is assumed to be $0.2 \pm 0.2$ for all $\Delta$ resonances in NEUT and $0.1 \pm 0.1$ $(0.2 \pm 0.2)$ for $\Delta^{++/-}\left(\Delta^{+/ 0}\right)$ resonances in NUANCE.

To determine the angular distribution of final state pions, the RS method [92] is used for the $P_{33}(1232)$ resonance in both NEUT and NUANCE. For other resonances, the directional distribution of the generated pion is chosen to be isotropic in the resonance rest frame.

The axial-vector form factors are formalized to be dipole with $M_{A}^{1 \pi}=1.21 \mathrm{GeV} / \mathrm{c}^{2}$ for NEUT and $M_{A}^{1 \pi}=1.10 \mathrm{GeV} / \mathrm{c}^{2}$ for NUANCE, with an uncertainty of $0.28 \mathrm{GeV} / \mathrm{c}^{2}$ in both cases.

An additional uncertainty is assigned to account for the observed $Q^{2}$ disagreement between the SciBooNE CC $1 \pi$-enriched data samples and NEUT [68. A similar disagreement is also observed for NUANCE. The size of the uncertainty is determined by re-weighting CC resonant pion events as a function of true $Q^{2}$ such that they match the observed distribution in the SciBooNE data, and using the difference between the re-weighted distribution and the central value as the uncertainty. A detailed description of this $Q^{2}$ shape error can be found in Appendix B.

Resonance decays leading to multi-pion final states are also included in the model and are simulated assuming $M_{A}^{N \pi}=1.30 \pm 0.52 \mathrm{GeV} / c^{2}$ in NUANCE. This value of $M_{A}^{N \pi}$ is chosen strictly to ensure that the total CC cross section prediction reproduces previous experimental data. In NEUT, multi-pion production is simulated as deep inelastic scattering as described later in Sec. 4.2.2.4, and the RS model is not used. The size of the systematic uncertainty is estimated based on $M_{A}^{N \pi}$ variations for NUANCE, and the same size error is assumed also for the NEUT prediction.

\subsubsection{Coherent pion production}

Coherent pion production is a neutrino interaction with a nucleus which remains intact, releasing one pion with the same charge as the incoming weak current. Because of the small momentum transfer to the target nucleus, the outgoing pion tends to be emitted in the forward direction, closely following the incoming neutrino direction. The formalism developed by Rein and Sehgal [93, 94] is used to simulate such interactions. The axial vector mass, $M_{A}^{c o h}$, is set to $1.0 \pm 0.28 \mathrm{GeV} / c^{2}$ in both NEUT and NUANCE. In NEUT, the total and inelastic pion-nucleon cross sections from the original Rein-Sehgal publication are employed [93, 94. In NUANCE, they are obtained from fits to PDG data [95] and implemented as a function of pion energy. Additionally, the NC and CC coherent pion production cross section predictions in NUANCE are rescaled by a factor of 0.65 to better match the measured rate of $\mathrm{NC}$ coherent $\pi^{0}$ production as measured in MiniBooNE [96]. 


\subsubsection{Deep inelastic scattering}

The deep inelastic scattering (DIS) cross section is calculated using the GRV98 parton distribution functions [97. Additionally, we have included the corrections in the small $Q^{2}$ region developed by Bodek and Yang [98] for both NEUT and NUANCE. The implementation of the model is slightly different in NEUT and NUANCE.

In NEUT, the DIS contribution is included for hadronic invariant masses $W>1.3 \mathrm{GeV} / c^{2}$. The pion multiplicity is additionally restricted to be greater than one for $1.3<W<2 \mathrm{GeV} / c^{2}$ to avoid double-counting sources of single pion production that are already included in the resonance portion of the simulation. The multi-hadron final states are simulated with two models in NEUT: a custom-made program [99] for events with $\mathrm{W}$ between 1.3 and $2.0 \mathrm{GeV} / c^{2}$ and PYTHIA/JETSET [100] for events with $\mathrm{W}$ larger than $2 \mathrm{GeV} / c^{2}$.

A restriction on pion multiplicity is not enforced by NUANCE. Instead, the DIS contribution slowly increases for $W$ values starting at $1.7 \mathrm{GeV}$ and becomes the only source of neutrino interactions above $W>2 \mathrm{GeV}$. This is done to create a smooth transition between the resonance and DIS models and ensure continuity in distributions of kinematics and hadron multiplicity in the region of overlap.

\subsubsection{Intra-nuclear interactions}

Following production, the intra-nuclear interactions of mesons and nucleons are simulated using a cascade model in which the particles are traced until they escape from the nucleus.

Although we only use kinematic information from the final state muon in this analysis, the simulation of intra-nuclear interactions is important since the pions/protons emitted from the nucleus can be mis-reconstructed as muons.

The inelastic scattering, charge exchange and absorption of pions in nuclei are simulated. For inelastic scattering and charge exchange interactions, the direction and momentum of pions are affected. In the scattering amplitude, Pauli blocking is also taken into account. A more detailed description of the intra-nuclear interaction simulations in NUANCE and NEUT can be found elsewhere [68, 73].

A $25 \%(30 \%)$ uncertainty in the overall pion absorption (charge exchange) cross section is assumed based on comparisons to pion-carbon scattering data 101-103. The uncertainty in proton re-scattering is estimated to be $10 \%$. Hence, we apply a $10 \%$ error on the number of proton tracks observed in SciBar. Additionally, we set a $20 \%$ uncertainty on the NC/CC ratio, estimated from the model dependence of the lepton-mass effect in the small $Q^{2}$ region.

\subsubsection{The expected number of neutrino events}

Table 4.3 summarizes the parameter choices used in NEUT and NUANCE for the comparisons presented here. We chose these parameters since they are the default parameter settings used in Super-K, K2K and T2K (NEUT) and MiniBooNE (NUANCE). The different parameter values result in different neutrino cross section predictions between the two.

With the SciBooNE neutrino beam exposure of $0.99 \times 10^{20}$ protons on target, the expected number of events in the SciBooNE detector for each neutrino interaction is listed in Table 4.4 , Because of the difference in parameter choices (Table 4.3), NEUT predicts a larger QE and single pion rate than NUANCE. The difference in QE rate is largely coming from the choice of $\kappa$ values, and the difference in single pion rate can be largely accounted for by the difference in $M_{A}^{1 \pi}$ assumptions. The major source of the factor of two larger multi-pion/DIS rate in NEUT compared to NUANCE is the difference of multi pion production simulation in the range $1.3<W<2.0 \mathrm{GeV}$; NEUT simulate these events as DIS, while NUANCE uses a resonant production model. These differences in cross section predictions between similar models with perfectly reasonable parameter choices further 
Table 4.3: Parameters used for neutrino interaction simulation.

\begin{tabular}{ccc}
\hline \hline Parameter & NEUT & NUANCE \\
\hline$p_{F}$ & $217 \mathrm{MeV}$ & $220 \mathrm{MeV}$ \\
$E_{B}$ & $25 \mathrm{MeV}$ & $34 \mathrm{MeV}$ \\
$M_{A}^{Q E}$ & $1.21 \mathrm{GeV}$ & $1.23 \mathrm{GeV}$ \\
$\kappa$ & 1.00 & 1.022 \\
$M_{A}^{1 \pi}$ & $1.21 \mathrm{GeV}$ & $1.10 \mathrm{GeV}$ \\
$M_{A}^{c o h}$ & $1.0 \mathrm{GeV}$ & $1.0 \mathrm{GeV}$ \\
$M_{A}^{N \pi}$ & $(\mathrm{DIS})$ & $1.3 \mathrm{GeV}$ \\
\hline \hline
\end{tabular}

highlight the inherent uncertainty in neutrino generator predictions and stress the importance of additional neutrino interaction measurements in this region.

Table 4.4: The expected number and fraction of events in each neutrino interaction estimated by NEUT and NUANCE at the SciBooNE detector location with the neutrino beam exposure of $0.99 \times 10^{20}$ protons on target. The 10.6 ton fiducial volume of the SciBar detector is assumed. CC and $\mathrm{NC}$ interactions are abbreviated as $\mathrm{CC}$ and $\mathrm{NC}$, respectively.

\begin{tabular}{lrrrr}
\hline \hline & \multicolumn{2}{c}{ NEUT } & \multicolumn{2}{c}{ NUANCE } \\
Interaction Type & \# Events & Fraction(\%) & \# Events & Fraction(\%) \\
\hline CC quasi-elastic & 53,038 & 41.5 & 47,573 & 43.6 \\
CC single $\pi$ via resonances & 29,452 & 23.0 & 25,863 & 23.7 \\
CC coherent $\pi$ & 1,760 & 1.4 & 1,736 & 1.6 \\
CC multi-pion, DIS, etc & 6,834 & 5.3 & 3,140 & 2.9 \\
NC total & 36,836 & 28.8 & 30,734 & 28.2 \\
\hline Total & 127,920 & 100.0 & 109,046 & 100.0 \\
\hline \hline
\end{tabular}

Figure 4.4 shows the expected number of total $\nu_{\mu} \mathrm{CC}$ interactions as a function of neutrino energy. One can see that the cross section prediction from NEUT is about $10-20 \%$ larger than that from NUANCE across the range of SciBooNE energies.

The expected number of $\nu_{\mu} \mathrm{CC}$ interactions in the 10.6 ton SciBar fiducial volume (Sec. 5.2 are $9.11 \times 10^{4}$ and $7.83 \times 10^{4}$ for the NEUT and NUANCE predictions, respectively.

\subsection{SciBooNE detector simulation}

Propagation of the final state particles from the target nucleus in the SciBooNE detector, and its responses are simulated using the GEANT4 framework. The detector simulation includes a detailed geometric model of the detector.

\subsubsection{Simulation of particle propagation in detector}

The Bertini cascade model within GEANT4 [104] is used to simulate the interactions of hadronic particles with detector materials. A $10 \%$ difference of the total pion-carbon cross section is seen for higher energy pions between the GEANT4 simulation and external measurements. Hence, we set $\pm 10 \%$ systematic uncertainty for the pion-carbon cross section. The systematic uncertainty of the energy deposit per unit length is estimated to be 3\% in SciBar and MRD, and 10\% in EC, which is dominated by the differences among various calculations of the range to energy conversion. 

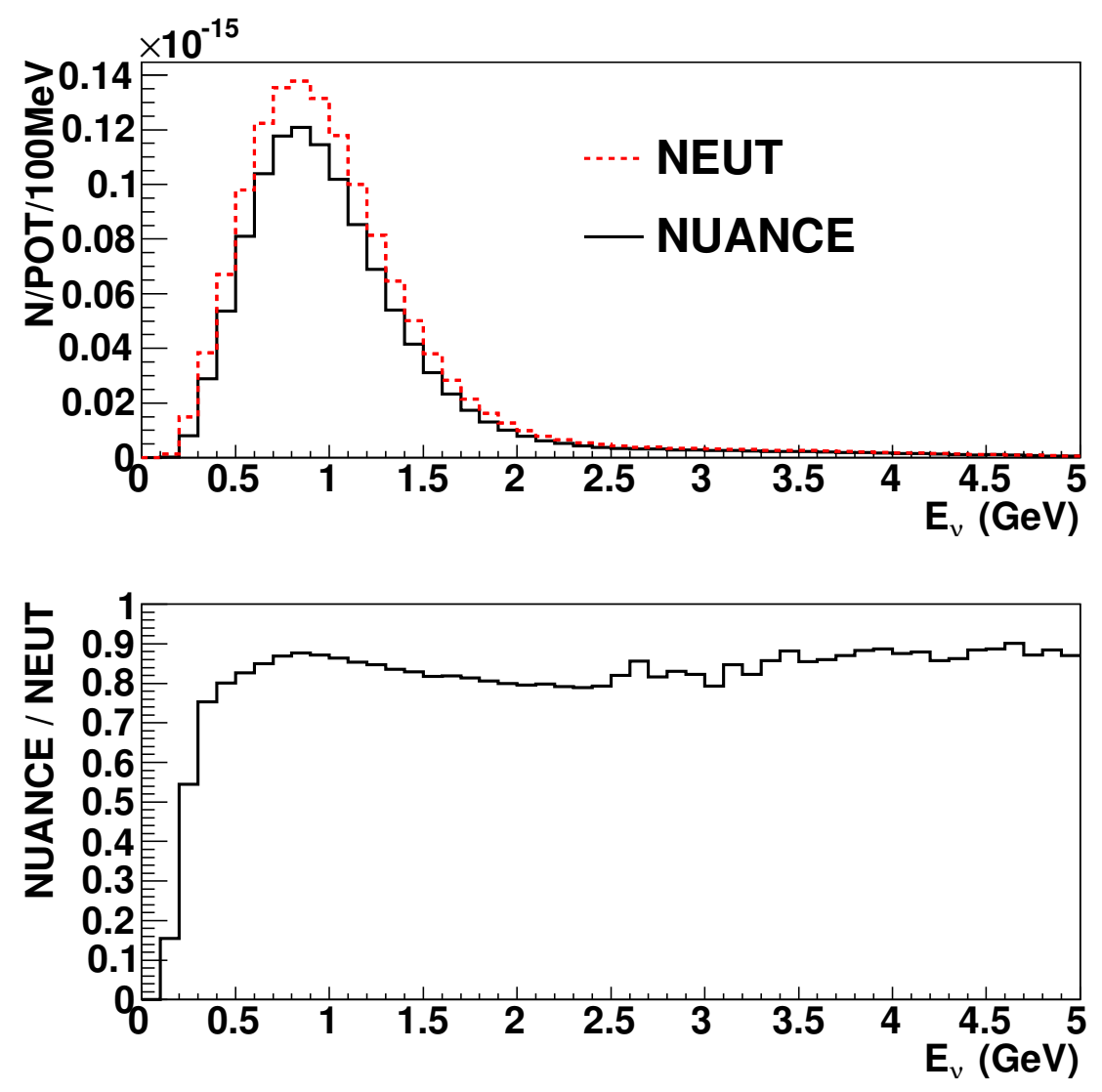

Figure 4.4: The neutrino energy distributions of $\nu_{\mu} \mathrm{CC}$ interactions at the SciBar detector generated by NEUT (red dashed line) and NUANCE (black solid line). Top plot is the number of interactions per unit POT, and the bottom plot shows the ratio between the NEUT and NUANCE predictions. 


\subsubsection{Simulation of detector response}

For the simulation of the SciBar detector, true energy depositions in scintillator strips simulated by GEANT4 are converted to the number of photo-electrons at the PMTs. The conversion factors are calculated based on the measured light yields for cosmic-ray muons and the gains of PMTs measured at laboratories. The scintillator quenching is simulated using Birk's law with the measured value of Birk's constant [105. The measured light attenuation length (Table 3.1) is used in the simulation. The crosstalk of the MA-PMT is measured to be $3.15 \pm 0.4 \%$ for adjacent channels and used for the simulation. The single photo-electron resolution of the MA-PMT is set to $50 \%$ in the simulation, to reproduce the observed $d E / d x$ distribution of cosmic muons. The absolute error is estimated to be $\pm 20 \%$. As for the detector mass, we measured the density of the scintillator by sampling $10 \%$ of the strips before the installation to be $1.021 \mathrm{~g} / \mathrm{cm}^{3}$ [106], and implemented into the simulation. The uncertainty of the total detector mass is estimated to be $1 \%$, including the effect of epoxy resin used to glue the strips.

For the detector simulation of the MRD, the time of energy deposition is digitized and converted into TDC counts. We surveyed the positions of steel plates and scintillator strips, and implemented to the simulation. Gaps between scintillator counters, which cause about $1 \%$ inefficiency, are also implemented.

A more detailed description of the detector simulation is given in 68].

\subsubsection{Neutrino interactions in the surrounding material}

In addition to neutrino interactions inside the detector, we also simulate interactions in the surrounding material (the walls of the detector hall and soil). The density of material is assumed to be $2.15 \mathrm{~g} / \mathrm{cm}^{3}$ for the calculation of the interaction rate. The concrete material with the density of $2.15 \mathrm{~g} / \mathrm{cm}^{3}$ is used as the material for propagation of product particles. We generate events in a volume of $\pm 5 \mathrm{~m}$ in $x, y$, and $z$ direction in the SciBooNE coordinates. The details are described in Ref. [77. 


\section{Chapter 5}

\section{Event reconstruction in SciBooNE detector}

To measure the rate of CC inclusive interactions, we use neutrino events occurring in SciBar with a muon in the final state. We select muons originating in the SciBar fiducial volume (FV), defined to be $\pm 130 \mathrm{~cm}$ in both the $x$ and $y$ dimensions, and $2.62<z<157.2 \mathrm{~cm}$, a total mass of 10.6 tons.

We describe the reconstruction of muon tracks in Sec. 5.1. the event selections and details the of sub-samples in Sec. 5.2 and comparison of the data to the MC predictions in Sec. 5.3 .

\subsection{Track reconstruction}

\subsubsection{Track finding}

Figure 5.1 shows the typical CC interaction candidate from the SciBooNE data.

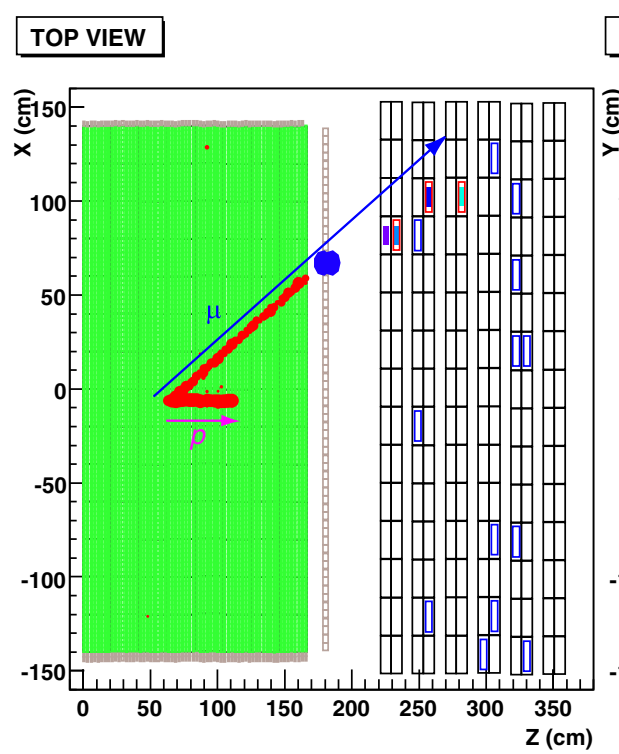
SIDE VIEW

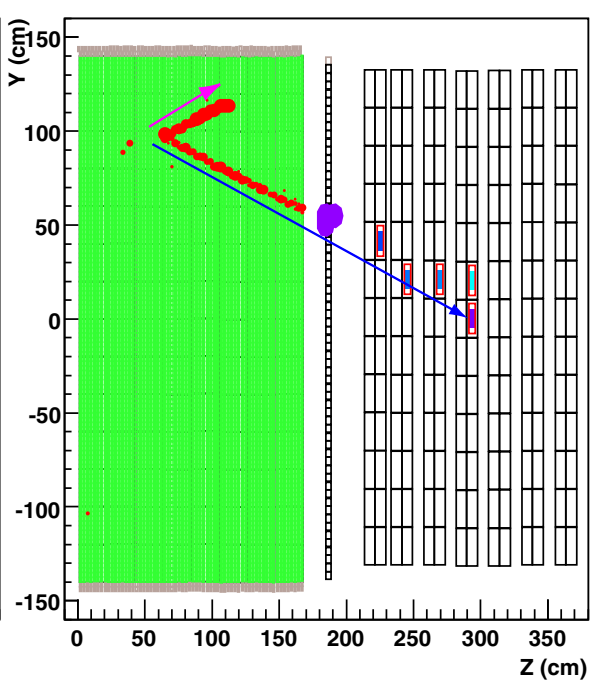

Figure 5.1: Event display of a typical muon neutrino charged current quasi-elastic scattering candidate from the SciBooNE data.

The first step of the event reconstruction is to search for two-dimensional tracks in each view of SciBar using a cellular automaton algorithm [107]. Three dimensional tracks are reconstructed by matching the timing and $z$-edges of the two dimensional projections; differences between two two-dimensional projections are required to be less than $50 \mathrm{~ns}$, and the $z$-edge difference must be 
less than $6.6 \mathrm{~cm}$ for both upstream and downstream edges. The two dimensional tracks in MRD are independently reconstructed using hits in the MRD clustered within a $50 \mathrm{~ns}$ timing window. Three dimensional tracks in the MRD are reconstructed by matching the timing of the two dimensional projections.

Then, if the downstream edge of a SciBar track lies in last two layers of SciBar, we search for a track or hits in the MRD that are matched with the SciBar track. For matching an MRD track to a SciBar track, the upstream edge of the MRD track is required to be on either one of the first two layers of the MRD, and to be within $30 \mathrm{~cm}$ of the projected entry point of the SciBar track into the MRD in each view. A SciBar track matched with MRD is defined as a SciBar-MRD matched track. The matching criteria impose a muon momentum threshold of $350 \mathrm{MeV} / c$. A more detailed description of the track reconstruction can be found elsewhere [68].

\subsubsection{Track classification}

We define three types of tracks used in this analysis: SciBar-stopped, MRD-stopped and MRDpenetrated tracks, as shown in Figure 5.2 .

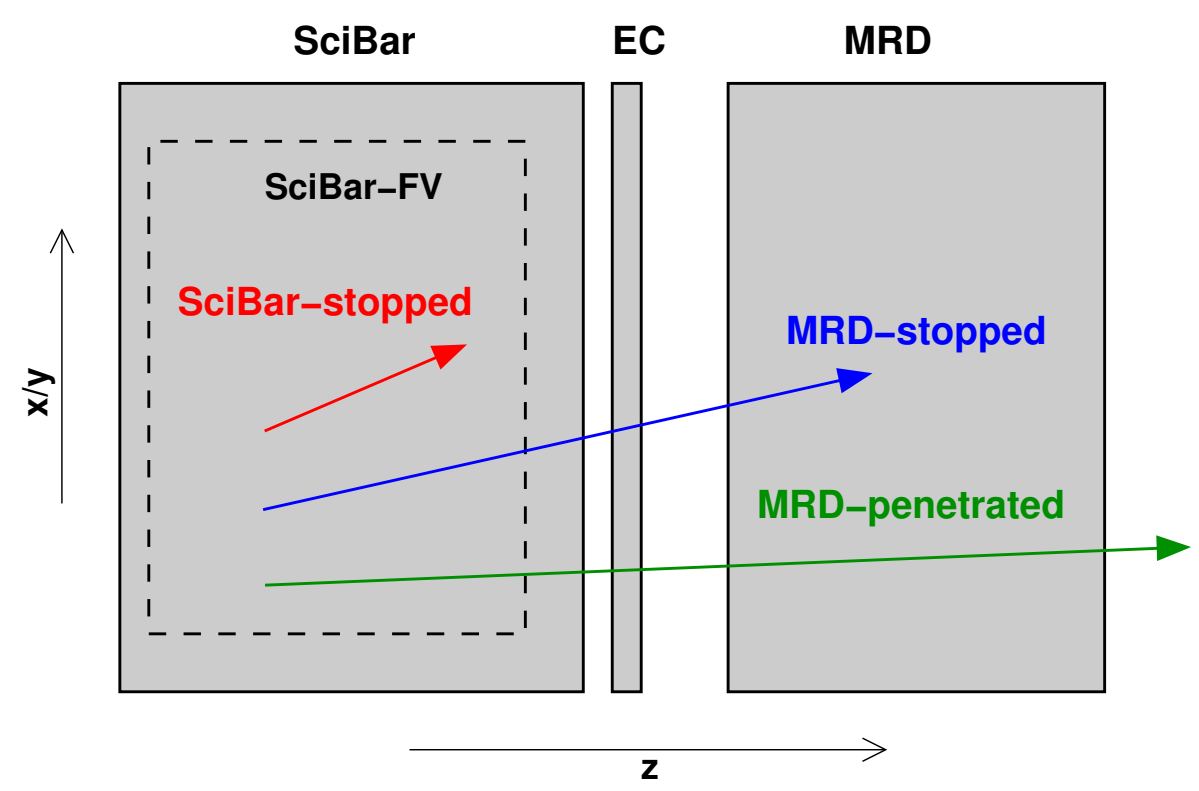

Figure 5.2: Types of tracks used for this CC interaction measurement.

Tracks with both edges contained in the SciBar FV are classified as SciBar-stopped tracks. MRD-stopped and MRD-penetrated tracks are both subset of the SciBar-MRD matched tracks. An MRD-stopped track is selected by requiring the downstream edge of the track to be within $|x|<132 \mathrm{~cm},|y|<111 \mathrm{~cm}$, and $z<$ (last layer of the MRD). An MRD-penetrated track is selected by requiring additional hits at the most downstream scintillator of the MRD. Tracks which exit from the side of the MRD are not used in this analysis.

\subsubsection{Particle identification}

The SciBar detector has the capability to distinguish protons from other particles using $d E / d x$. We define a muon confidence level (MuCL) using the observed energy deposit per layer for all reconstructed tracks [68. Tracks with MuCL greater than 0.05 are considered muon-like (or pionlike) and the others are classified as proton-like. 


\subsubsection{Muon kinematics reconstruction}

\subsubsection{Muon angle reconstruction}

The slopes of the muon angles with respect to the beam in the two SciBar views are used to calculate the three dimensional muon angle with respect to the beam $\left(\theta_{\mu}\right)$.

According to the MC simulation, about $30 \%$ of SciBar-stopped tracks are expected to be backward-going. To identify these backward-going tracks, we use the delayed timing signal produced by the decay electrons from stopped muons. Figures 5.3 and 5.4 are timing distributions of the delayed hits at the upstream and downstream edge of SciBar tracks.
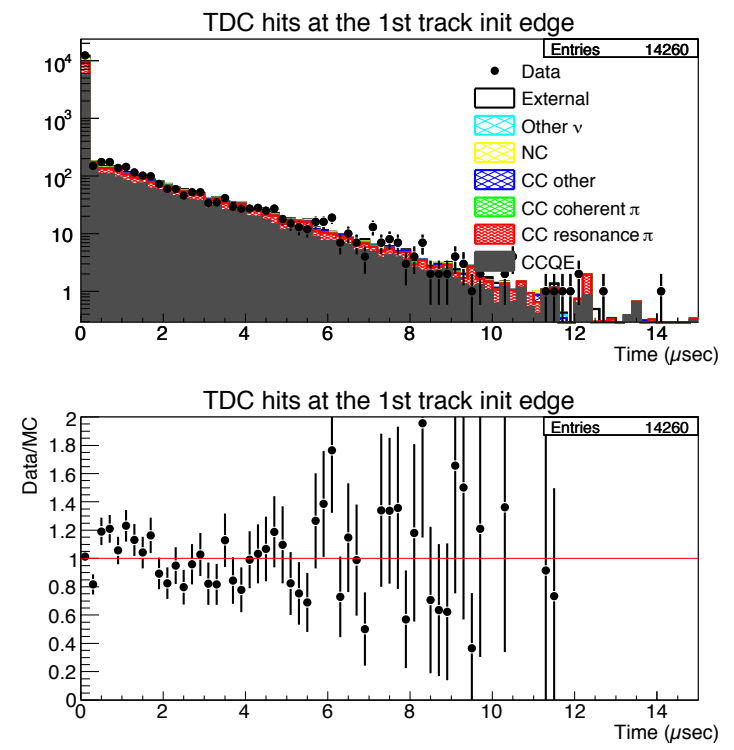

Figure 5.3: Timing distribution of hits on TDC at the upstream edge of the SciBar stopped track.
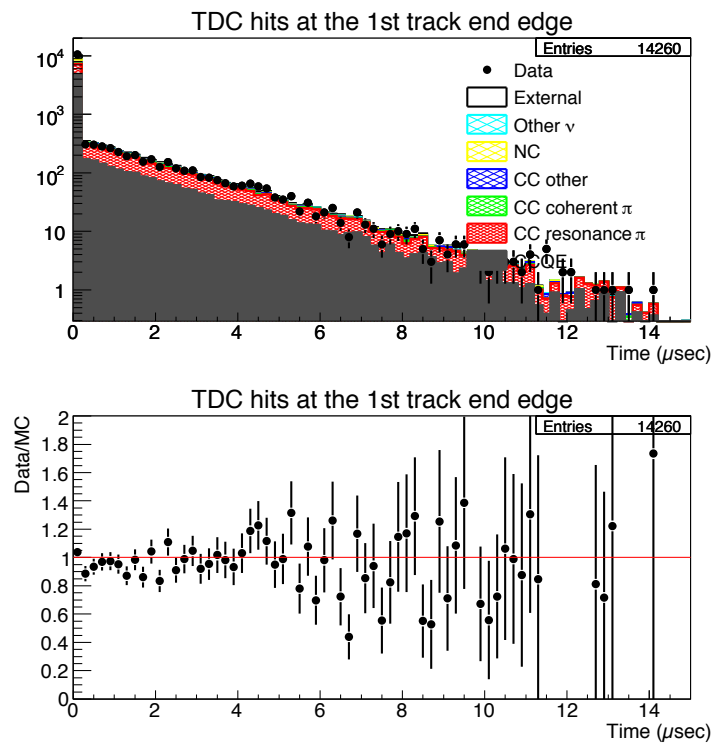

Figure 5.4: Timing distribution of hits on TDC at the downstream edge of the SciBar stopped track.

A track is identified to be backward-going if it has

- At least one delayed hit at $t>200 \mathrm{nsec}$ at the upstream ends of the two dimensional views, and

- No delayed hit at $t>200$ nsec at the downstream ends.

We impose this requirement for both top- and side- PMT signals from the track endpoints to remove hits due to random noise and after pulsing from the PMTs. The performance of this identification is estimated using a MC sample of CCQE events with 1 reconstructed track. In this sample, the efficiency of selecting backward-going tracks is $\sim 57 \%$, and the probability of misreconstructing a forward track as backward is $\sim 1 \%$. The loss of efficiency is predominantly due to decay electrons emitted at a large angle, producing hits in either the top or side PMTs, but not both.

This track direction identification is only applied to SciBar-stopped tracks. All tracks reaching the MRD are assumed to be forward-going, because the fraction of backward tracks is estimated to be small $(\sim 4 \%)$ and also a similar tagging of decay electrons is not possible in the MRD since the electrons stop and are undetected within the steel plates.

The average muon angular resolution is $0.9^{\circ}$ for all samples. 


\subsubsection{Muon momentum reconstruction}

The kinetic energy of the muon $\left(E_{k i n}\right)$ is calculated by the range and the expected energy deposition per unit length $(d E / d x)$ in the detector materials

$$
E_{k i n}=E^{S c i B a r}+E^{E C}+E^{\text {Wall }}+E^{M R D}
$$

where $E^{S c i B a r}, E^{E C}, E^{W a l l}$ and $E^{M R D}$ are the expected energy deposit by muons in SciBar, the $\mathrm{EC}$, the wall of the dark box between the EC and MRD, and the MRD, respectively.

For SciBar-stopped tracks, $E^{E C}, E^{W a l l}$ and $E^{M R D}$ are set to 0 , and $E^{\text {SciBar }}$ is calculated by a range to energy look-up table based on the MC simulation. Figure 5.5 shows the range to energy conversion table for SciBar-stopped tracks. The expected muon momentum resolution is shown in the Figure 5.6. The resolution is much better compared to MRD-stopped sample since we can measure the muon range more precisely for SciBar-stopped tracks.

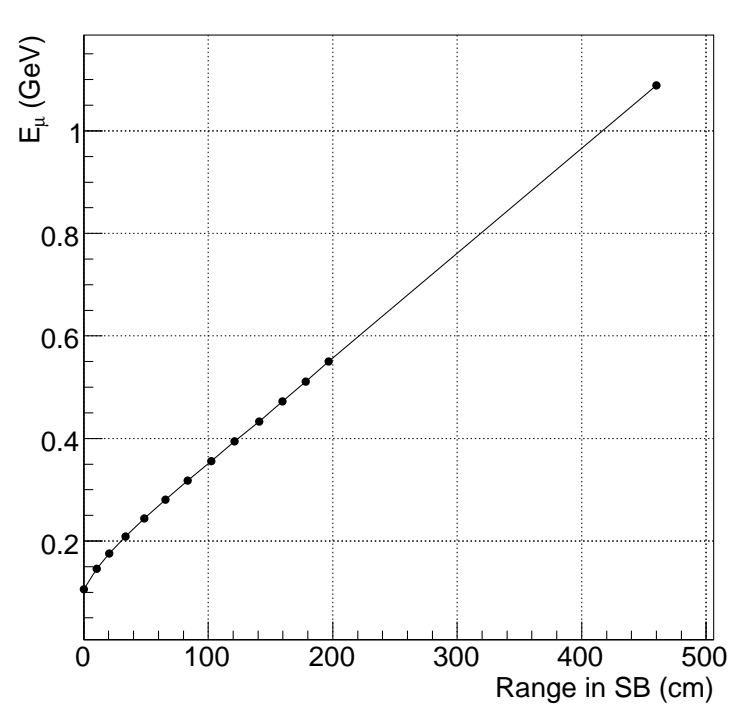

Figure 5.5: Range to muon energy conversion table used to reconstruct $E_{\mu}$ for SciBar-stopped tracks.

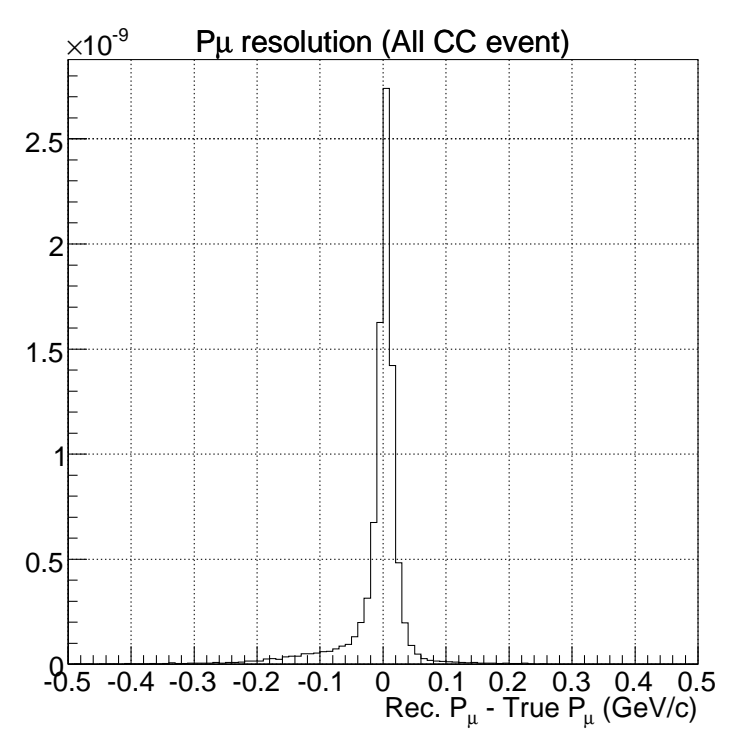

Figure 5.6: Muon momentum resolution for SciBar stopped tracks.

For MRD-stopped tracks, energy deposits in SciBar, EC and the wall are computed as $E^{\text {SciBar }}=$ $2.04 \mathrm{MeV} / \mathrm{cm} \times L_{S B}(\mathrm{~cm}), E^{E C}=90.8 / \cos \theta_{\mu} \mathrm{MeV}$ and $E^{\text {Wall }}=3.3 / \cos \theta_{\mu} \mathrm{MeV}$, where $L_{S B}$ is the reconstructed track length in SciBar. $E^{M R D}$ is calculated by a range to energy look-up table based on the MC simulation. The range-to-energy conversion factor used to compute $E_{\mu}^{M R D}$ is shown in the Figure 5.7. Figure 5.8 shows the expected muon momentum resolution for MRD-stopped muons.

The muon momentum resolutions are $15 \mathrm{MeV} / \mathrm{c}$ for SciBar-stopped and $50 \mathrm{MeV} / \mathrm{c}$ for MRDstopped tracks, respectively.

\subsection{Event selection and classification}

\subsubsection{Event selection}

We select the highest momentum track with MuCL $>0.05$ in an event as a muon candidate. The trick timing $t$ is required to be within $0<t<2 \mu s e c$, to remove backgrounds from accidental events such as cosmic rays. We also require the reconstructed momentum to be greater than $0.25 \mathrm{GeV} / \mathrm{c}$ to reject short proton or pion tracks from neutral current interactions. Then, we require the upstream edge of the muon candidate to be in the SciBar FV. 


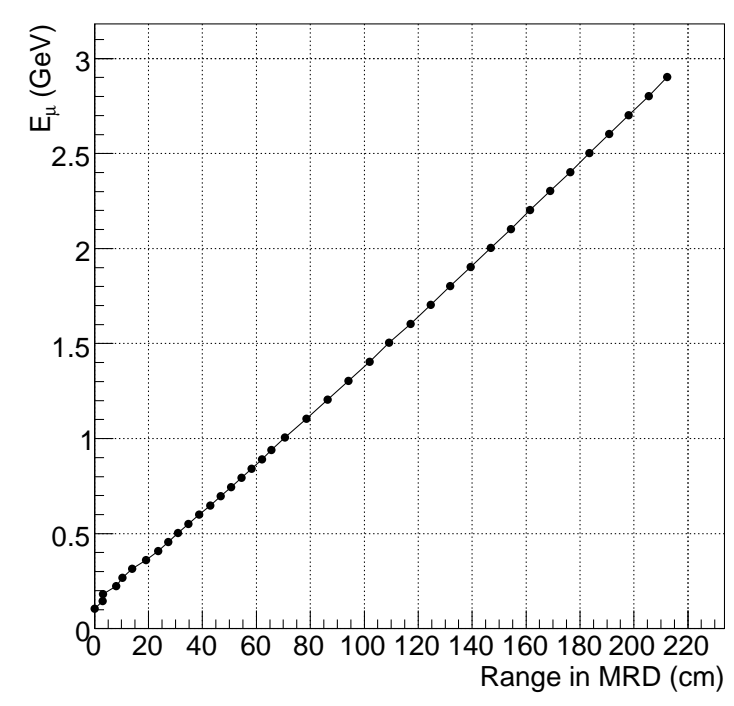

Figure 5.7: Range to muon energy conversion table used to reconstruct $E_{\mu}^{M R D}$.

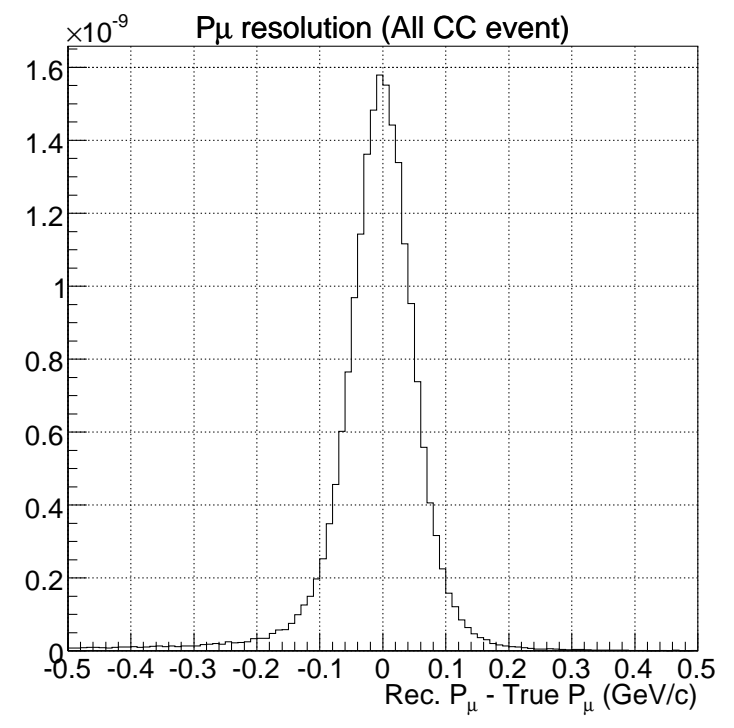

Figure 5.8: Muon momentum resolution for MRD-stopped tracks.

\subsubsection{Validity of the $p_{\mu}$ cut}

As described above, we require that $p_{\mu}>0.25 \mathrm{GeV} / \mathrm{c}$ for the $\mathrm{CC}$ event selection. Here, we discuss the effect and the validity of this cut. This cut is especially important for the SciBar-stopped tracks, which suffered by the large contamination of backgrounds from $\mathrm{NC}$ interaction and the neutrino interaction in the dirt. Figure 5.9 shows the muon momentum distribution before applying " $p_{\mu}$ " cut. We can see the large portion of NC and Dirt events are removed by this " $p_{\mu}$ " requirement. The other motivation of $p_{\mu}$ requirement comes from MiniBooNE sample. They require (the number of tanks hits) $>200$, to reject the Michel electron signals. Figure 5.10 shows the true $p_{\mu}$ distribution of MiniBooNE final $\nu_{\mu}$ CC-QE sample used for the oscillation fit. From this figure, we can see the tank hits threshold corresponds to $p_{\mu} \sim 0.25 \mathrm{GeV} / \mathrm{c}$. Hence, we do not gain sensitivity to the oscillation analysis by lowering the $p_{\mu}$ threshold below $0.25 \mathrm{GeV} / \mathrm{c}$.

Additionally, the validity of MuCL requirement is checked since the MuCL is tuned for high momentum muons. Figure 5.11 shows the distribution of MuCL vs. $p_{\mu}$ for true muon tracks in SciBar-stopped sample. Although the current MuCL tends to return 0 for low momentum muons, it is safe to use once we require $p_{\mu}>0.25 \mathrm{GeV} / \mathrm{c}$.

\subsubsection{Event classification}

Events with muon track candidates are subdivided into three sub-samples: SciBar-stopped, MRDstopped and MRD-penetrated samples, according to the track classification given in Sec. 5.1.2 for the muon candidate.

\subsubsection{SciBar-stopped sample}

The SciBar-stopped sample provides the lowest energy sample; the mean energy of neutrinos in this sample is $1.0 \mathrm{GeV}$. According to the simulation, the purity of $\nu_{\mu} \mathrm{CC}$ interactions in this sample is $85 \%$. Impurities are due to $\nu_{\mu} \mathrm{NC}$ interactions $(\sim 7 \%)$, interactions occurring in the surrounding material $(\sim 5 \%)$ and $\bar{\nu}_{\mu}$ CC interactions $(\sim 1.5 \%)$. Figure 5.12 shows the distributions of the reconstructed muon momentum $\left(p_{\mu}\right)$ and angle $\left(\theta_{\mu}\right)$ for SciBar-stopped muons. The expected number of events in each interaction mode is summarized in Table 5.1. The contamination of the cosmic-ray backgrounds is estimated, using off-beam data, to be $671.2 \pm 11.6$ events, where the 

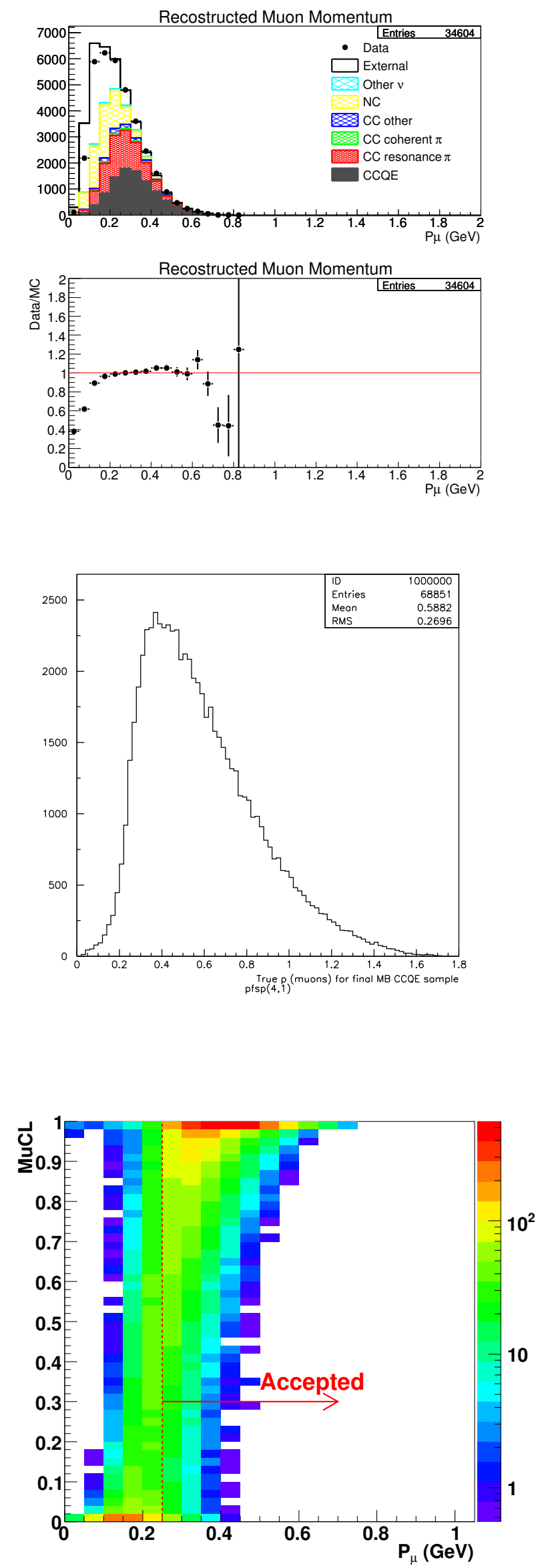

Figure 5.9: Reconstructed muon momentum after applying base cuts except for $p_{\mu}$ and $\mathrm{MuCL}$ cuts. Events with $p_{\mu}>0.25 \mathrm{GeV}$ are selected.

Figure 5.10: True muon momentum of MiniBooNE final $\nu_{\mu}$ CC-QE sample used for the oscillation fit.

Figure 5.11: The distributions of MuCL vs. $p_{\mu}$ for true muon tracks in SciBar-stopped sample. The color represent the number of event in logarithm scale. 
uncertainty comes from the statistics of the off-beam data sample. This rather large contamination $(\sim 5 \%)$ is due to cosmic-rays which penetrate SciBar vertically, leaving sparse hits which can be misreconstructed as short tracks. This effect is negligible for the other subsamples.
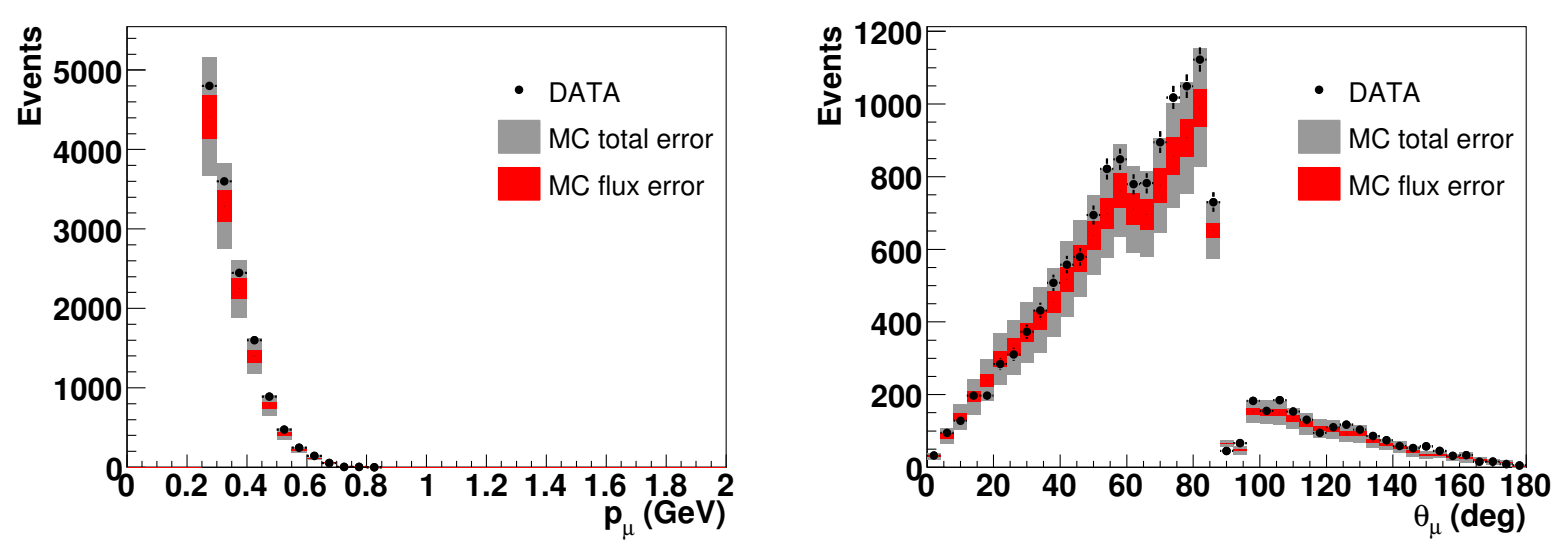

Figure 5.12: Distributions of reconstructed momentum (left) and angle (right) of the muon candidate in the SciBar-stopped sample. The MC prediction is based on NEUT and absolutely normalized by the number of POT. The total and flux systematic errors on the MC predictions are separately shown. The dominant source of the total error is the cross section uncertainty.

Table 5.1: The expected number and fraction of events in each neutrino interaction for the SciBar-stopped sample, estimated by NEUT and NUANCE.

\begin{tabular}{lrrrr}
\hline \hline Interaction & \multicolumn{2}{c}{ NEUT } & \multicolumn{2}{c}{ NUANCE } \\
type & Events & Fraction(\%) & Events & Fraction(\%) \\
\hline CC QE & 5818 & 47.4 & 5511 & 50.8 \\
CC res. $1 \pi$ & 3694 & 30.1 & 3226 & 29.8 \\
CC coh. $1 \pi$ & 119 & 1.0 & 123 & 1.1 \\
CC other & 914 & 7.4 & 350 & 3.2 \\
NC & 916 & 7.4 & 842 & 7.8 \\
All non- $\nu_{\mu}$ & 188 & 1.5 & 161 & 1.5 \\
External & 629 & 5.1 & 629 & 5.8 \\
\hline Total & 12278 & & 10,842 & \\
\hline \hline
\end{tabular}

\subsubsection{MRD-stopped sample}

The MRD-stopped sample has the largest statistics among the three. The mean energy of neutrinos in the MRD-stopped sample is $1.2 \mathrm{GeV}$. According to the simulation, the purity of $\nu_{\mu} \mathrm{CC}$ interaction in this sample is $91 \%$. Impurities are from neutrino interactions in the EC/MRD which backscatter $(\sim 4 \%), \nu_{\mu} \mathrm{NC}$ interactions $(\sim 3 \%)$ and $\bar{\nu}_{\mu} \mathrm{CC}$ interactions $(\sim 2 \%)$. Figure 5.13 shows the distributions of the reconstructed muon momentum $\left(p_{\mu}\right)$ and angle $\left(\theta_{\mu}\right)$ for MRD-stopped muons. The expected number of events in each interaction mode is summarized in Table 5.2. The contamination of the cosmic-ray backgrounds is estimated to be $54.6 \pm 3.3$ events. 

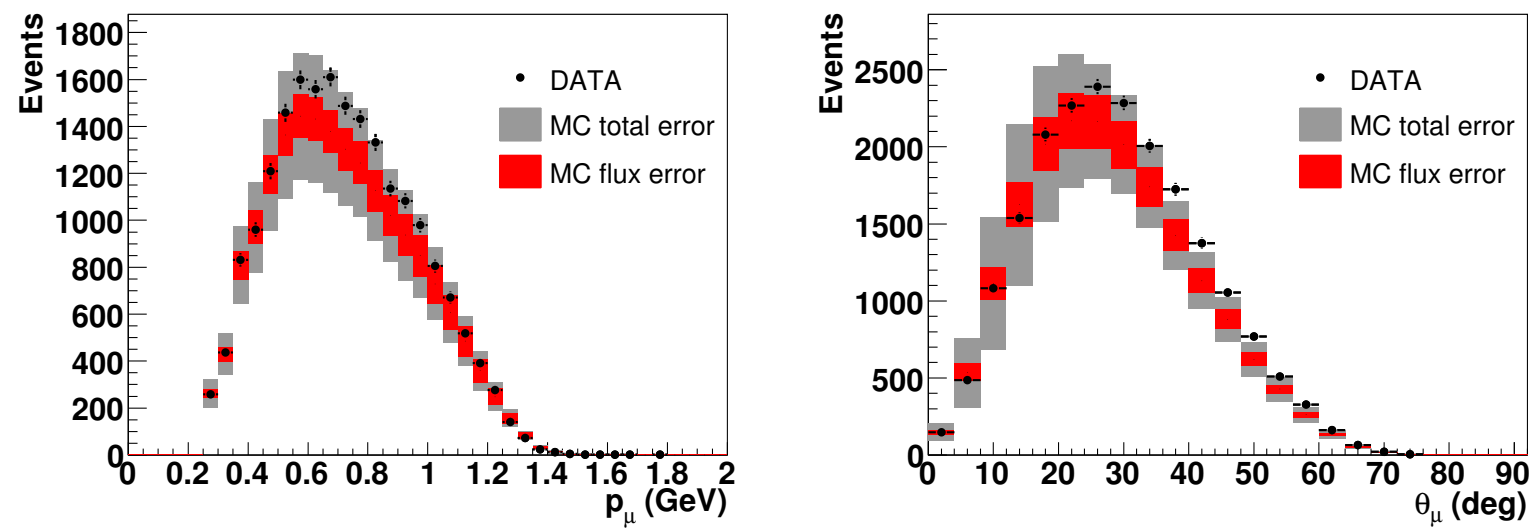

Figure 5.13: Distributions of reconstructed momentum (left) and angle (right) of the muon candidate in the MRD-stopped sample. The MC prediction is based on NEUT and absolutely normalized by the number of POT. The total and flux systematic errors on the MC predictions are separately shown. The dominant source of the total error is the cross section uncertainty.

Table 5.2: The expected number and fraction of events in each neutrino interaction for the MRDstopped sample, estimated by NEUT and NUANCE.

\begin{tabular}{lrrrr}
\hline \hline Interaction & \multicolumn{2}{c}{ NEUT } & \multicolumn{2}{c}{ NUANCE } \\
type & Events & Fraction(\%) & Events & Fraction(\%) \\
\hline CC QE & 10341 & 56.1 & 8385 & 52.3 \\
CC res. $1 \pi$ & 4789 & 26.0 & 4839 & 30.2 \\
CC coh. $1 \pi$ & 659 & 3.6 & 633 & 3.9 \\
CC other & 1010 & 5.5 & 600 & 3.7 \\
NC & 577 & 3.1 & 569 & 3.5 \\
All non- $\nu_{\mu}$ & 320 & 1.7 & 281 & 1.8 \\
External & 729 & 4.0 & 729 & 4.5 \\
\hline Total & 18,427 & & 16,036 & \\
\hline \hline
\end{tabular}




\subsubsection{MRD-penetrated sample}

The MRD-penetrated sample is the highest energy sample among the three. The mean energy of neutrinos is $2.4 \mathrm{GeV}$. Although the track angles can be measured, we do not have the capability to reconstruct the muon momentum for the tracks which exit the MRD. However, this sample can provide the normalization for the highest energy region. Hence, this sample is also used for the neutrino interaction rate measurement. According to the simulation, the purity of $\nu_{\mu}$ CC interaction in this sample is $97 \%$. Impurities mostly come from $\bar{\nu}_{\mu}$ CC interactions $(\sim 2 \%)$. Figure 5.14 shows the distributions of the reconstructed muon angle $\left(\theta_{\mu}\right)$ of the MRD-penetrated muons. The expected number of events in each interaction mode is summarized in Table 5.3. The contamination of the cosmic-ray backgrounds is estimated to be $16.6 \pm 1.8$ events.

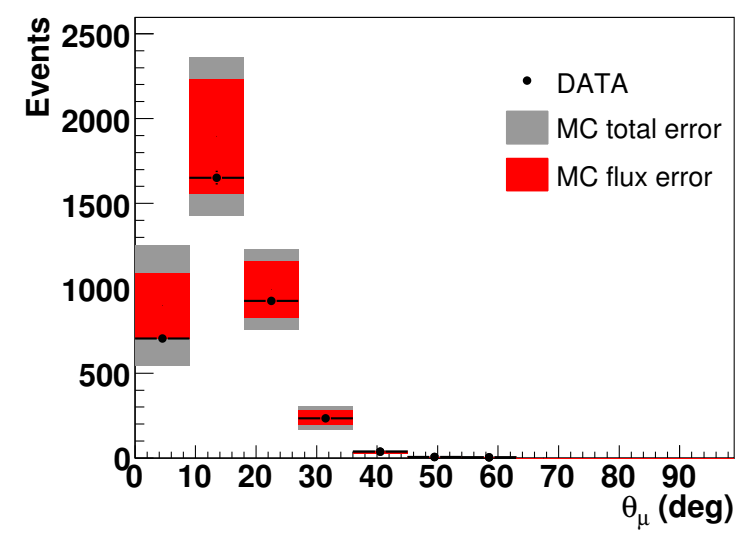

Figure 5.14: Distribution of reconstructed angle of the muon candidate in the MRD-penetrated sample. The MC prediction is based on NEUT and absolutely normalized by the number of POT. The total and flux systematic errors on the MC predictions are separately shown.

Table 5.3: The expected number and fraction of events in each neutrino interaction for the MRDpenetrated sample, estimated by NEUT and NUANCE.

\begin{tabular}{lrrrr}
\hline \hline Interaction & \multicolumn{2}{c}{ NEUT } & \multicolumn{2}{c}{ NUANCE } \\
type & Events & Fraction(\%) & Events & Fraction(\%) \\
\hline CC QE & 2428 & 60.0 & 1943 & 57.0 \\
CC res. $1 \pi$ & 1008 & 24.9 & 976 & 28.6 \\
CC coh. $1 \pi$ & 140 & 3.5 & 130 & 3.8 \\
CC other & 356 & 8.8 & 255 & 7.4 \\
NC & 1.5 & 0.04 & 2.3 & 0.07 \\
All non- $\nu_{\mu}$ & 89 & 2.2 & 75 & 2.2 \\
External & 27 & 0.7 & 27 & 0.8 \\
\hline Total & 4049 & & 3407 & \\
\hline \hline
\end{tabular}

\subsubsection{Efficiency summary}

Figures 5.15 and 5.16 shows the efficiency of CC events as a function of true $P_{\mu}$ and $\theta_{\mu}$. Figure 5.17 shows the efficiency of $\mathrm{CC}$ events as a function of true neutrino energy for each sub-sample, estimated from the NEUT based MC simulation. By combining these three samples, we can obtain fairly uniform efficiency for neutrinos above $0.4 \mathrm{GeV}$. 

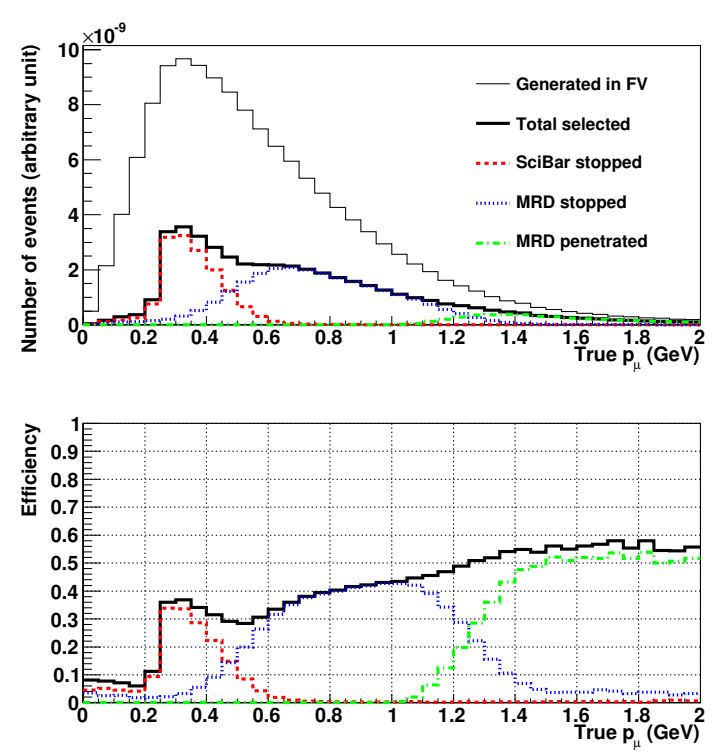

Figure 5.15: (Top) Number of CC events in the SciBar FV as a function of true $p_{\mu}$, predicted by the NEUT based simulation. The number of selected events in each sub-sample are also shown. (Bottom) Detection efficiency as a function of true neutrino $p_{\mu}$ for each subsample.
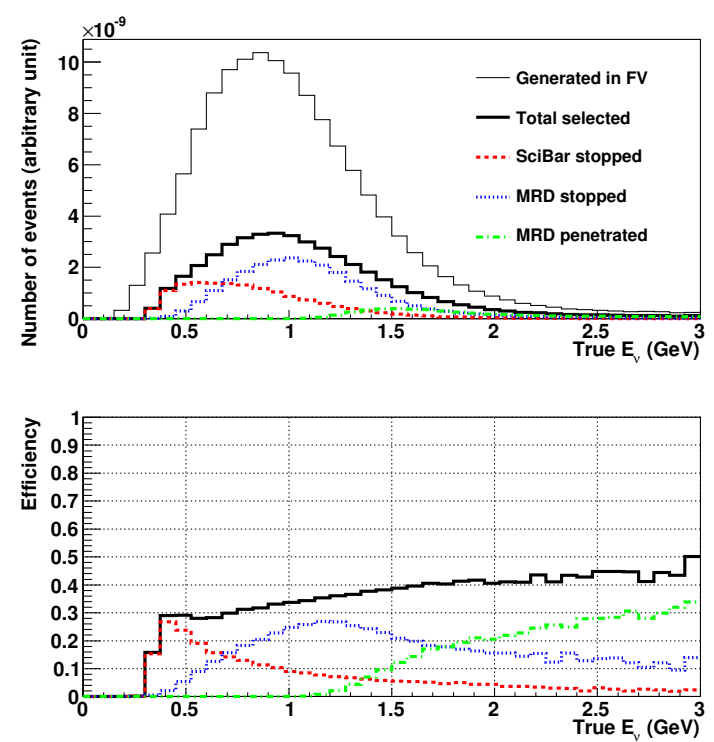
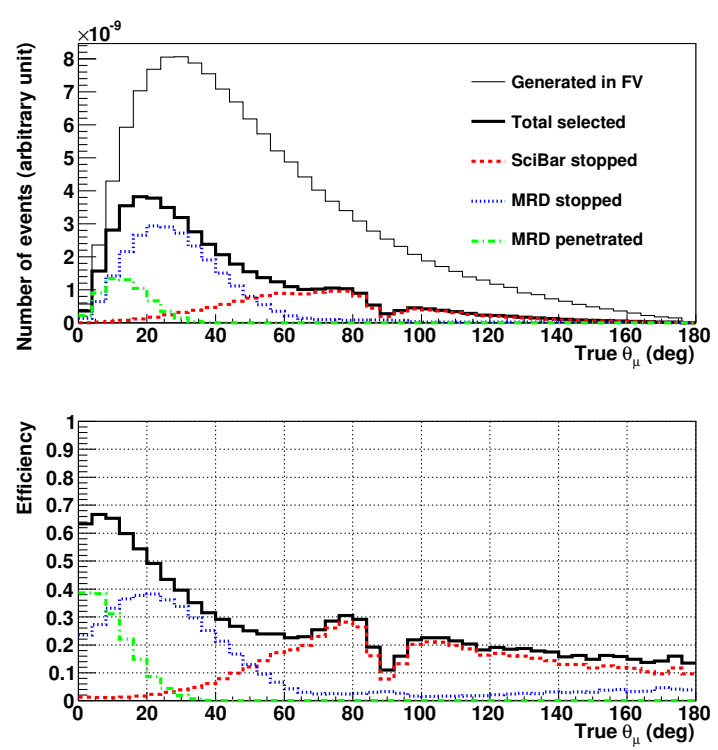

Figure 5.16: (Top) Number of CC events in the SciBar FV as a function of true $\theta_{\mu}$, predicted by the NEUT based simulation. The number of selected events in each sub-sample are also shown. (Bottom) Detection efficiency as a function of true neutrino $\theta_{\mu}$ for each subsample.

Figure 5.17: (Top) Number of CC events in the SciBar FV as a function of true $E_{\nu}$, predicted by the NEUT based simulation. The number of selected events in each sub-sample are also shown. (Bottom) Detection efficiency as a function of true neutrino energy for each sub-sample. 


\subsection{Data comparison to the MC prediction}

Table 5.4 shows the number of events obtained from data and the predictions from NEUT and NUANCE based MC simulations. The contamination of cosmic-ray backgrounds is estimated using the off-beam data, and have been subtracted from the data. For the total number of events from the three sub-samples, we find a data/MC normalization factor of 1.08 for the NEUT prediction, and 1.23 for the NUANCE prediction. The systematic errors of the number of the sum of these three samples are estimated to be 0.07 from the flux uncertainty, and 0.16 from the cross-section uncertainty. Hence the observed normalization differences are fairly covered by the systematic uncertainties.

Table 5.4: The number of events in each sub-sample from the data and the predictions from NEUT/NUANCE-based MC. The numbers in parentheses show the ratio between the data and the predictions. The cosmic-ray backgrounds are estimated from off-timing data and subtracted from the data.

\begin{tabular}{l|ccc|c}
\hline \hline Sample & SciBar-stopped & MRD-stopped & MRD-penetrated & Total \\
\hline Data & 13588.8 & 20236.4 & 3544.4 & 37369.6 \\
NEUT & $12278.3(1.11)$ & $18426.3(1.10)$ & $4049.0(0.88)$ & $34753.6(1.08)$ \\
NUANCE & $10841.9(1.25)$ & $16036.2(1.26)$ & $3407.5(1.04)$ & $30285.6(1.23)$ \\
\hline \hline
\end{tabular}

To compare the MC predictions with data in more detail, the neutrino energy $\left(E_{\nu}\right)$ and the square of the four-momentum transfer $\left(Q^{2}\right)$ are the key variables since a flux variation is purely a function of $E_{\nu}$ while a variation of the cross section model typically changes the $Q^{2}$ distribution. We reconstruct these variables assuming CC-QE interaction kinematics. The reconstructed $E_{\nu}$ is calculated as

$$
E_{\nu}^{r e c}=\frac{m_{p}^{2}-\left(m_{n}-E_{B}\right)^{2}-m_{\mu}^{2}+2\left(m_{n}-E_{B}\right) E_{\mu}}{2\left(m_{n}-E_{B}-E_{\mu}+p_{\mu} \cos \theta_{\mu}\right)},
$$

where $m_{p}, m_{n}$ and $m_{\mu}$ are the mass of proton, neutron and muon, respectively, $E_{\mu}$ is the muon total energy, and $E_{B}$ is the nuclear potential energy. The reconstructed $Q^{2}$ is given by,

$$
Q_{r e c}^{2}=2 E_{\nu}^{r e c}\left(E_{\mu}-p_{\mu} \cos \theta_{\mu}\right)-m_{\mu}^{2} .
$$

Figure 5.18 shows the distributions of $E_{\nu}^{r e c}$ and $Q_{r e c}^{2}$ for the SciBar-stopped and MRD-stopped samples. In these plots, data points are compared with the NEUT and NUANCE based MC predictions. We find that the data are consistent with the MC predictions within the systematic uncertainties. 

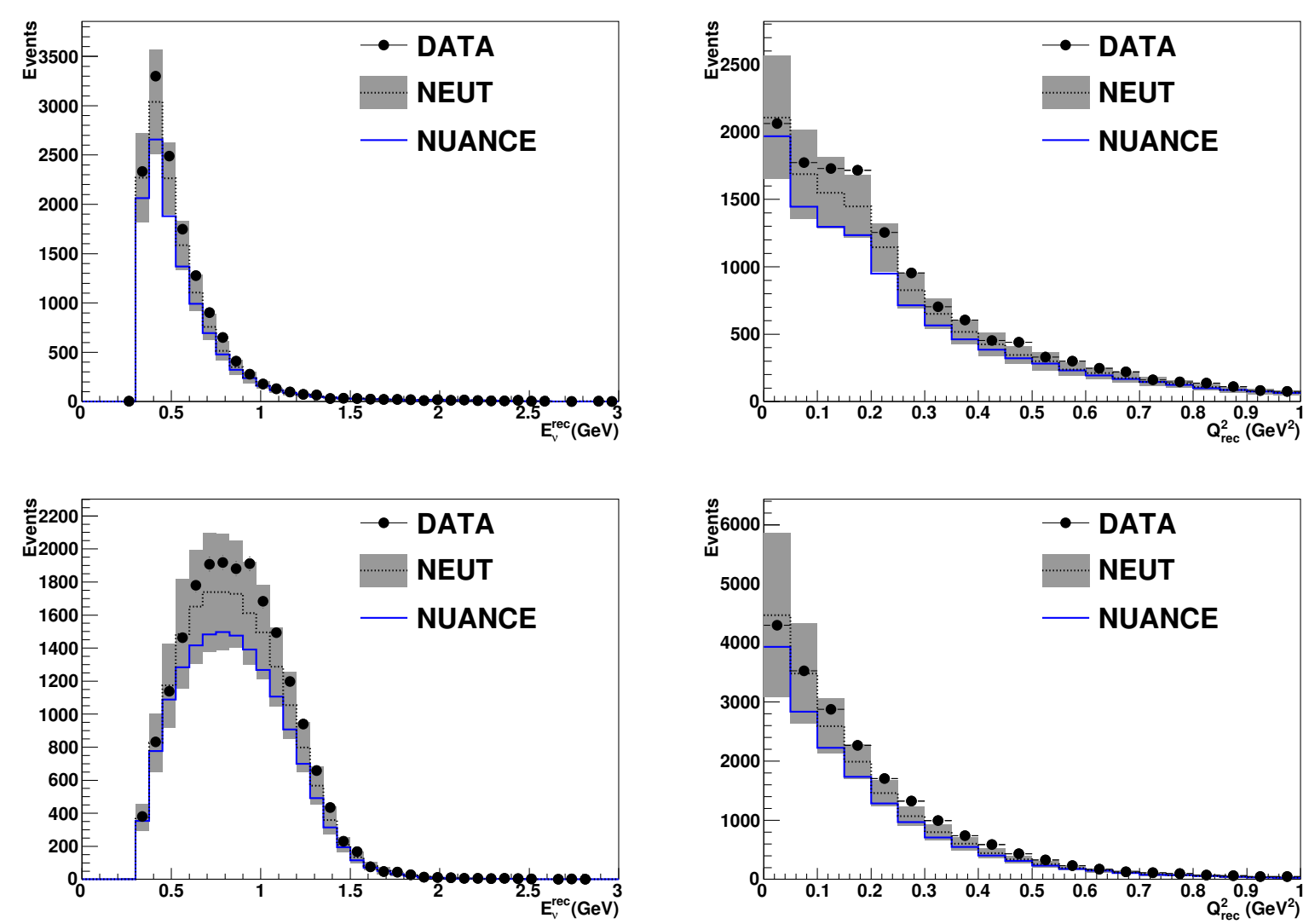

Figure 5.18: Top: $E_{\nu}^{r e c}$ (left) and $Q_{r e c}^{2}$ (right) of the SciBar-stopped sample. Bottom: $E_{\nu}^{r e c}$ (left) and $Q_{r e c}^{2}$ (right) of the MRD-stopped sample. The NEUT and NUANCE predictions are absolutely normalized by the number of POT. The filled regions show the systematic uncertainties on the MC predictions based on NEUT. The systematic uncertainty for the NUANCE prediction is similar to that of the NEUT prediction and not shown. 


\section{Chapter 6}

\section{Measurement of CC interactions at SciBooNE}

\subsection{Overview}

In this section, we report the measurements of neutrino charged current interaction rate and cross section at SciBooNE. This is the first measurement of CC inclusive interaction cross section in the $1 \mathrm{GeV}$ region on carbon. Hence this result will help selecting the various cross section models and tuning the neutrino flux prediction.

In addition, we also discuss the applications of this measurement to other measurements at SciBooNE and MiniBooNE. The first application is providing the absolute normalization for the previous cross section measurements at SciBooNE, and the second is providing a constraint of the product of the flux and cross section for a SciBooNE-MiniBooNE joint $\nu_{\mu}$ disappearance analysis.

This chapter is organized as follows. First, we describe the method to extract energy dependent $\mathrm{CC}$ interaction rate in Sec. 6.2. Then, we give the results of CC interaction rate and cross section measurement in Sec. 6.3. We also discuss the applications of this result to other measurements in Sec. 6.4. The summary of this chapter is given in Sec. 6.5.

\subsection{CC interaction rate analysis}

\subsubsection{Method}

To measure the CC inclusive interaction rate and the cross section as a function of neutrino energy, we re-weight the predictions of NEUT and NUANCE based simulations in true energy bins by factors that give the best agreement with the kinematic distributions for data with the MC prediction.

The $p_{\mu}$ vs. $\theta_{\mu}\left(p_{\mu}-\theta_{\mu}\right)$ distributions from the SciBar-stopped and the MRD-stopped samples, and $\theta_{\mu}$ distribution from the MRD-penetrated sample are used for this measurement. Figure6.1 shows the $p_{\mu}-\theta_{\mu}$ distributions of the SciBar-stopped and MRD-stopped samples, while the $\theta_{\mu}$ distribution for the MRD-penetrated sample is shown in Fig. 5.14. Events in the same $p_{\mu}-\theta_{\mu}$ bins but in different sub-samples are not summed together, but treated as separate $p_{\mu}-\theta_{\mu}$ bins in the analysis, and only bins with at least 5 entries are used for the fit. The total number of $p_{\mu^{-}} \theta_{\mu}$ bins is $159 ; 71$ from the SciBar-stopped, 82 from the MRD-stopped and 6 from the MRD-penetrated samples.

We define six rate normalization factors $\left(f_{0}, \cdots, f_{5}\right)$ which represent the CC interaction rate normalized to the MC prediction for each true energy region defined in Table 6.1. If the default $\mathrm{MC}$ prediction is equal to the observed data, the value of $f_{i}$ is 1 for $i=0, \cdots 5$. The events at $E_{\nu}<0.25 \mathrm{GeV}$ are not used since these events are below our detection threshold as shown in Fig. 5.17, and also the fraction of these low energy interactions are negligibly small $(<1 \%)$ at the 
BNB flux. We calculate these rate normalization factors by comparing the MC predictions to the measured $\mathrm{CC}$ interaction rate.

More discussions of the choice of samples and binning is described in Appendix A.1.
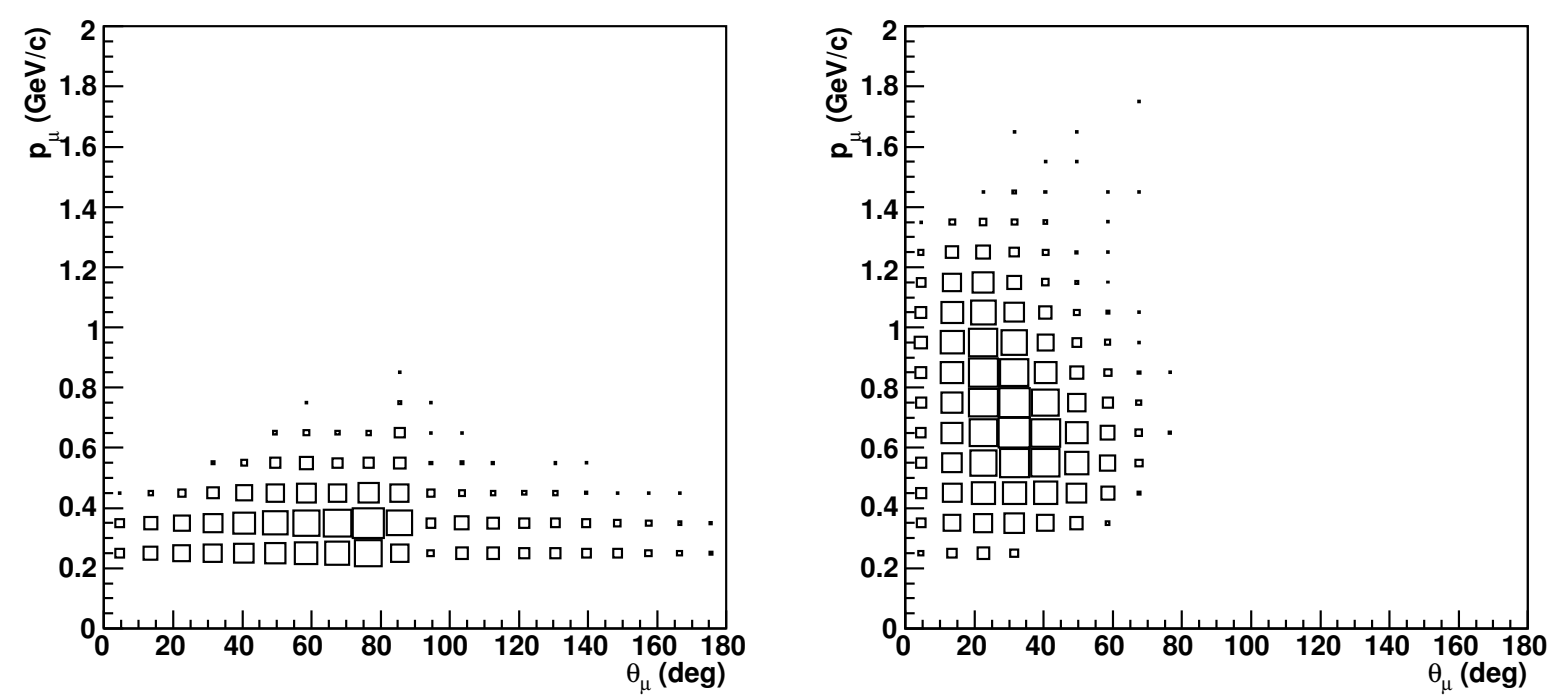

Figure 6.1: Data distributions of $p_{\mu}$ vs. $\theta_{\mu}$ for the SciBar-stopped (left) and MRD-stopped (right) samples. The size of boxes is proportional to the number of entries.

Table 6.1: Energy regions for the CC interaction rate measurement. These energy regions are in terms of the true neutrino energy from the MC.

\begin{tabular}{lcccccc}
\hline \hline Parameter & $f_{0}$ & $f_{1}$ & $f_{2}$ & $f_{3}$ & $f_{4}$ & $f_{5}$ \\
\hline$E_{\nu}$ range $(\mathrm{GeV})$ & $0.25-0.5$ & $0.5-0.75$ & $0.75-1.0$ & $1.0-1.25$ & $1.25-1.75$ & $1.75+$ \\
\hline \hline
\end{tabular}

First, we generate the MC templates for the $p_{\mu}-\theta_{\mu}$ distributions in each event sample; $n_{i j}^{\text {pred }}$ is the predicted number of events in the i-th true energy bin and $\mathrm{j}$-th $p_{\mu}-\theta_{\mu}$ bin. Then, the expected number of events in each $p_{\mu}-\theta_{\mu}$ bin, $N_{j}^{p r e d}$, is calculated as

$$
N_{j}^{\text {pred }}=\sum_{i}^{E_{\nu} \text { bins }} f_{i} n_{i j}^{\text {pred }} .
$$

Figures 6.2 and 6.3 are the MC templates of the $p_{\mu}-\theta_{\mu}$ distributions for the SciBar-stopped and MRD-stopped samples. We see that there is a large contribution in the SciBar-stopped sample of events with $E_{\nu}$ below $0.75 \mathrm{GeV}$. Hence, this sample is essential to determine the rate normalization factors in the low energy regions. The $p_{\mu}-\theta_{\mu}$ distributions of the MRD-stopped sample clearly depends on $E_{\nu}$, up to $1.75 \mathrm{GeV}$. For the events with $E_{\nu}$ above $1.75 \mathrm{GeV}$, most of muons from $\mathrm{CC}$ interaction penetrate the entire MRD, and hence categorized to the MRD-penetrated sample. However, there is small portion of events with $E_{\nu}>1.75 \mathrm{GeV}$ in the MRD-stopped sample which have small reconstructed $p_{\mu}$. These are events with energetic pion or proton tracks that are misreconstructed as muons. Due to the weak constraint from the MRD-stopped sample on events with $E_{\nu}>1.75 \mathrm{GeV}$, the MRD-penetrated sample is included in the fit since about $2 / 3$ of the events in this sample have $E_{\nu}>1.75 \mathrm{GeV}$ as shown in Fig. 6.4. 

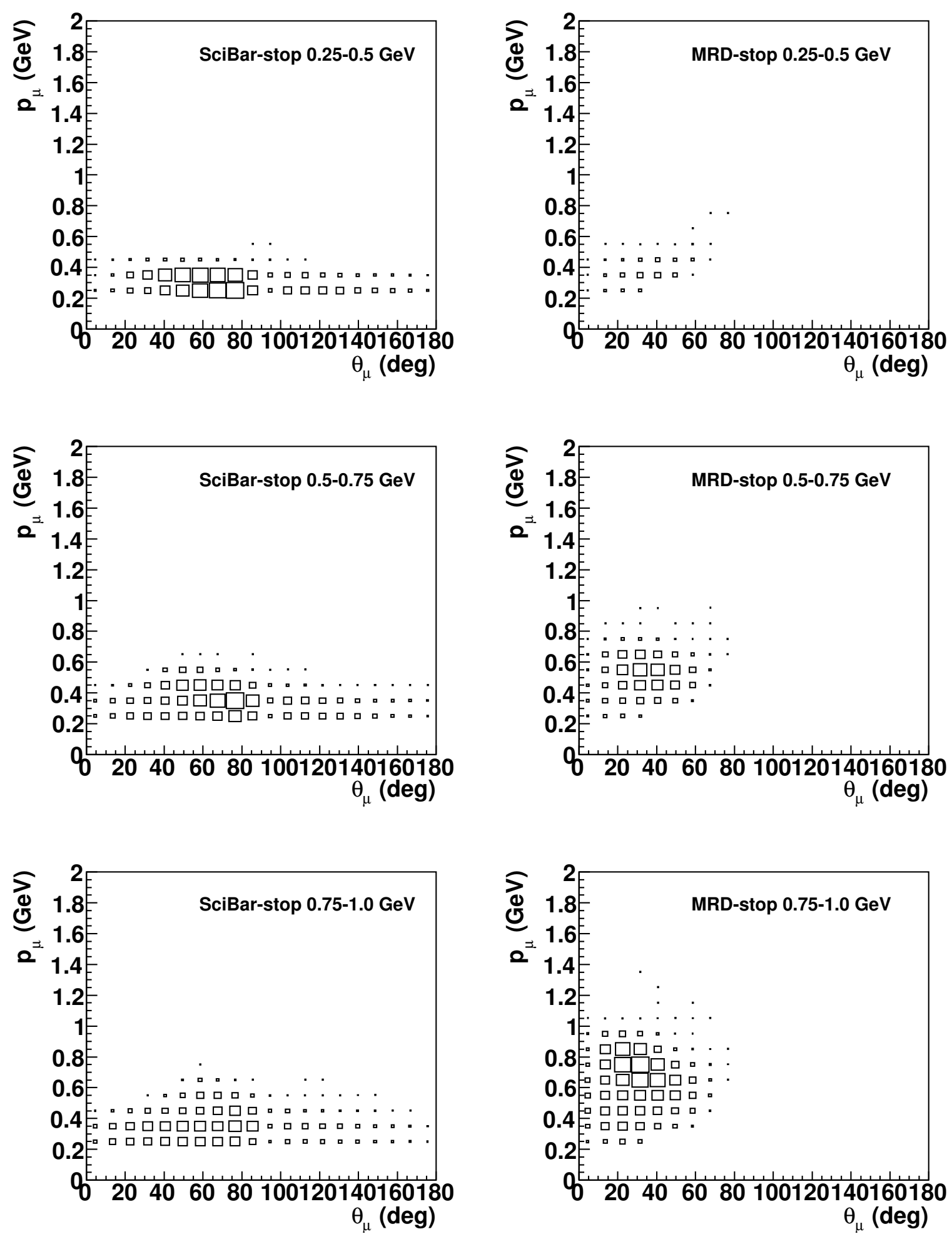

Figure 6.2: The MC templates of $p_{\mu}$ vs. $\theta_{\mu}$ for the SciBar-stopped and MRD-stopped sample for the three lowest $E_{\nu}$ regions. The normalization factors are common between the SciBar-stopped and MRD-stopped samples. 

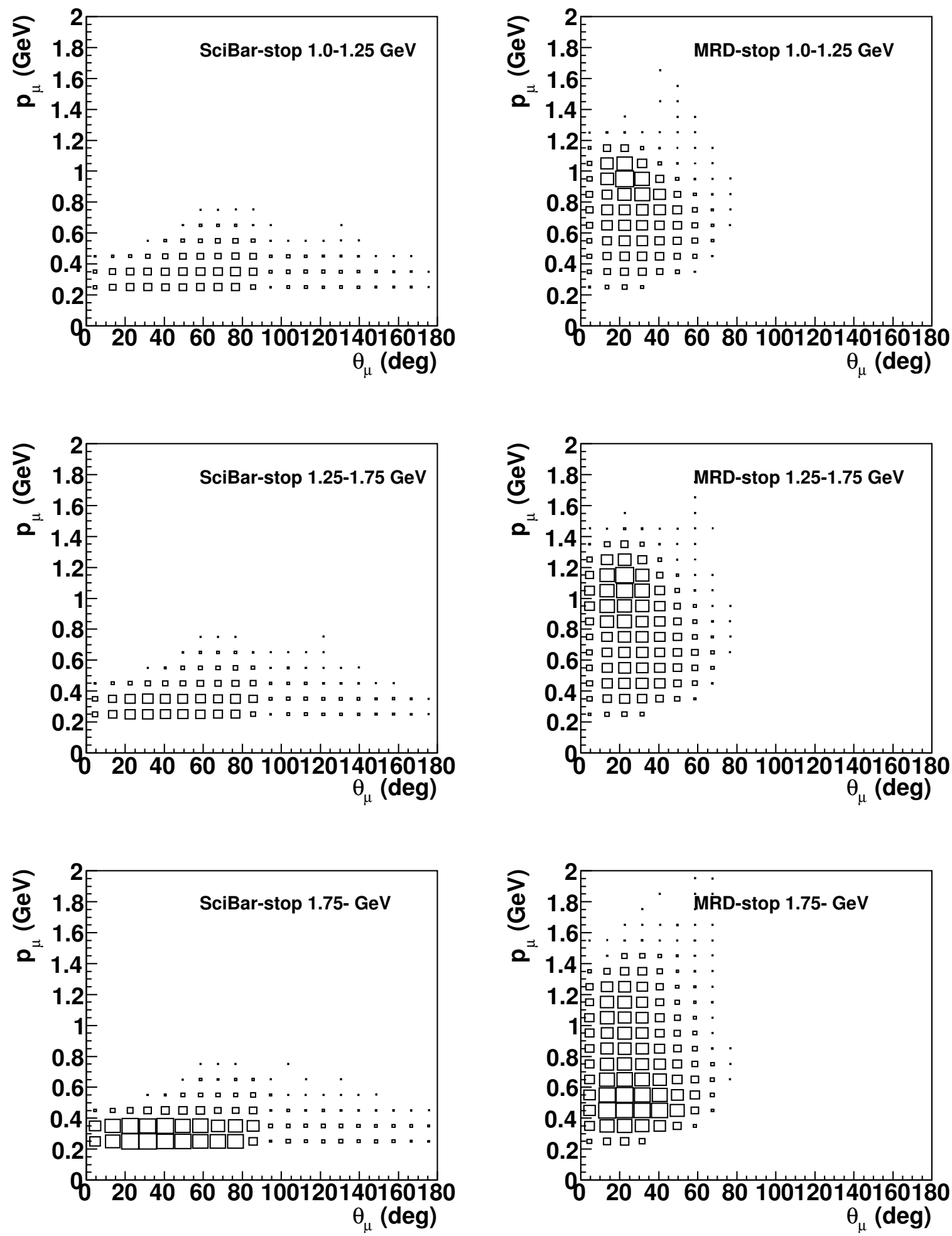

Figure 6.3: The MC templates of $p_{\mu}$ vs. $\theta_{\mu}$ for the SciBar-stopped and MRD-stopped sample for the three highest $E_{\nu}$ regions. The normalization factors are common between the SciBar-stopped and MRD-stopped samples. 


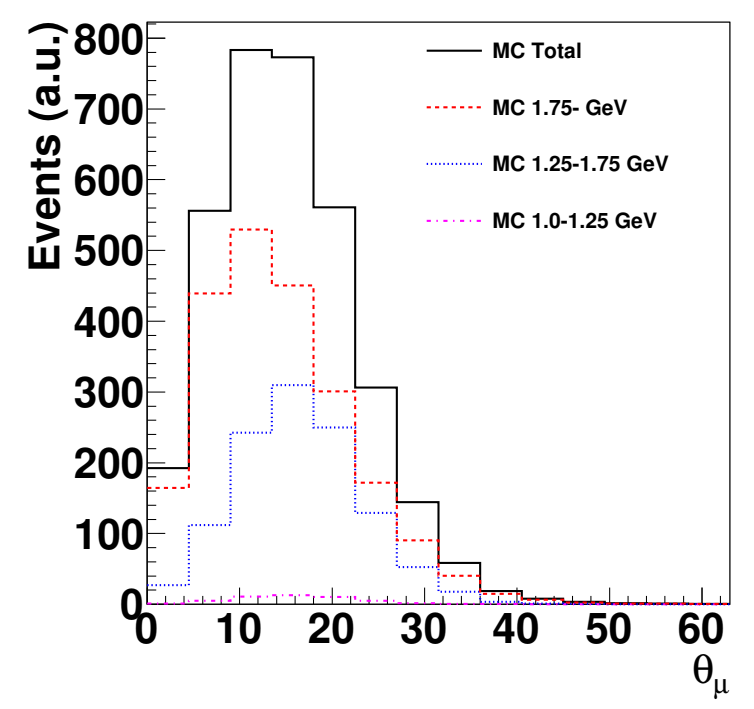

Figure 6.4: The MC templates of $\theta_{\mu}$ for the MRD-penetrated sample. The black line shows the total MC prediction. The predictions for $E_{\nu}>1.75 \mathrm{GeV}, 1.25<E_{\nu}<1.75 \mathrm{GeV}$ and $1.0<E_{\nu}<$ $1.25 \mathrm{GeV}$ are also shown. 


\subsubsection{Definition of $\chi^{2}$}

We search for the rate normalization factors $\left(f_{0}, \cdots, f_{5}\right)$ which minimize the Pearson's $\chi^{2}$ value defined as:

$$
\chi^{2}=\sum_{j, k}^{N b i n s}\left(N_{j}^{o b s}-N_{j}^{\text {pred }}\right)\left(V^{\text {sys }}+V^{\text {stat }}\right)_{j k}^{-1}\left(N_{k}^{o b s}-N_{k}^{\text {pred }}\right) .
$$

We refer this process as the "spectrum fit". Here, $N_{j(k)}^{o b s}$ and $N_{j(k)}^{\text {pred }}$ are the observed and predicted numbers of events in the $j(k)$-th $p_{\mu}-\theta_{\mu}$ bin, and $N_{j(k)}^{p r e d}$ is a function of the rate normalization factors as shown in Eq. 6.1). $\mathbf{V}^{\text {sys }}$ is the covariance matrix for systematic uncertainties in each $p_{\mu}-\theta_{\mu}$ bin, and $\mathbf{V}^{\text {stat }}$ represents the statistical error, defined as:

$$
\mathbf{V}^{\text {stat }}=\left(\begin{array}{cccc}
N_{1}^{\text {pred }} & & & 0 \\
& N_{2}^{\text {pred }} & & \\
& & \ddots & \\
0 & & & N_{\text {Nbins }}^{\text {pred }}
\end{array}\right)
$$

We have a total of 159 bins, so $\mathbf{V}^{\text {sys }}$ and $\mathbf{V}^{\text {stat }}$ are $159 \times 159$ dimensional matrices. The details of evaluating $\mathbf{V}^{\text {sys }}$ are described in the following section.

This method assumes that the statistical fluctuation follows the Gaussian distribution around the predation $N_{i}^{M C}$, while the Poisson distribution is more accurate approximation for low statistic bins. We have tested the two assumptions and found that the fit using the Gaussian distribution produces the consistent result with the fit assuming the Poisson distribution. Then, because the fit process is the Pearson's $\chi^{2}$ is a lot simpler and stabler compare to the likelihood method, we decided to use the Pearson's $\chi^{2}$ for the fit. The details of the comparison are described in the Appendix A.2.

\subsubsection{Error analysis}

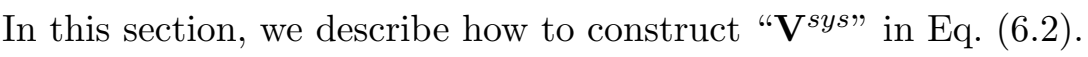

\subsubsection{Method}

The sources of systematic errors are divided into four categories: the uncertainties of neutrino beam (i), neutrino interaction models (ii), intra-nuclear interaction model (iii), and detector response and neutrino interaction models outside of SciBar (iv). Table 6.2 shows the list of the systematic uncertainties considered in this analysis, which were described in the previous sections.

The general procedure of producing the error matrix of $p_{\mu}-\theta_{\mu}$ distribution $\left(\mathbf{V}^{\text {sys }}\right)$ is as follows. For each sources listed in Table 6.2, we randomly change the parameters (like $M_{A}$ ) and produce a corresponding $p_{\mu}-\theta_{\mu}$ distribution, which we call a "systematic variation". We produce this systematic variation many times for each source, and compare these with the original prediction. Then, from the difference between the systematic variations and the original prediction, we form the error matrix of $p_{\mu}-\theta_{\mu}$ distribution, including the correlations between the bins. 


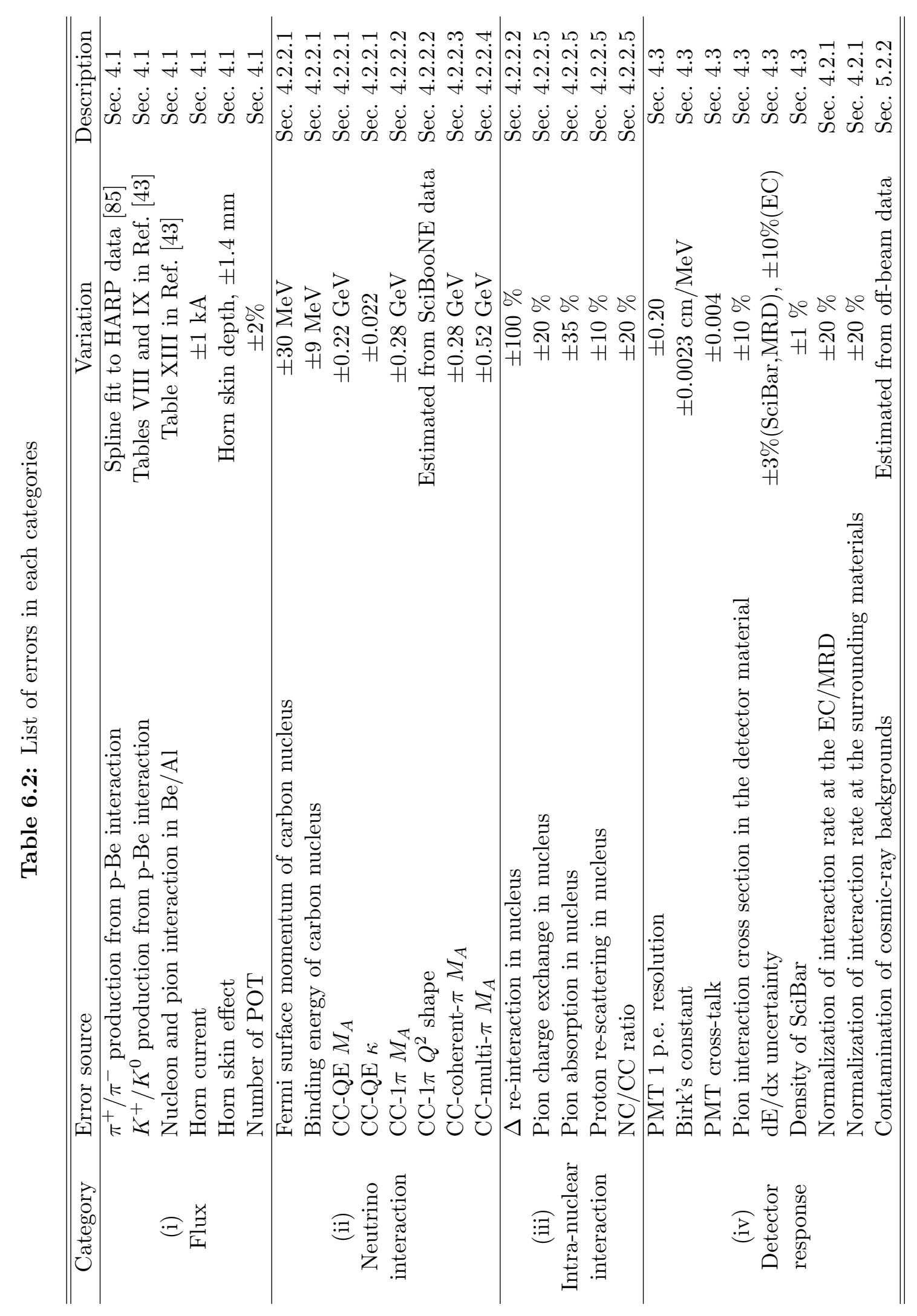


The aim of this analysis is to constrain the variations due to the flux (i) and the cross section (ii) by measuring the rate normalization factors as a function of $E_{\nu}, f_{i}$. Uncertainties from (i) and (ii) are factorized into two parts: variations which change the rate normalization of CC events in each $E_{\nu}$ region ("normalization") and variations which change the $p_{\mu}-\theta_{\mu}$ distributions but not the normalization of CC events (" $p_{\mu}-\theta_{\mu}$ shape"). The former, normalization, is corrected by the use of $f_{i}$, so $V_{\text {sys }}$ corresponds only to $p_{\mu}-\theta_{\mu}$ shape uncertainties by the flux and the cross section.

The $p_{\mu}-\theta_{\mu}$ shape uncertainties are estimated by re-normalizing the variation for each $E_{\nu}$ region as follows. First, we generate a new prediction corresponding to a systematic variation of $p_{\mu}-\theta_{\mu}$ distribution, $n_{i j}^{\prime}$. Here, the prime denotes a systematic variation, with $i$ and $j$ representing as before the energy bin $i$ and $p_{\mu}-\theta_{\mu}$ bin $j$.

Then, the predicted event rate, $N_{j}^{\prime}$, corresponding to this systematic variation is:

$$
N_{j}^{\prime}=\sum_{i}^{E_{\nu} \text { bins }} f_{i} n_{i j}^{\prime} R_{i},
$$

where $R_{i}$ is the renormalization factor, which is the ratio of the total number of predicted events in each $E_{\nu}$ bin between the default prediction and the systematically varied one defined as:

$$
R_{i}=\frac{\sum_{j}^{\left(p_{\mu}, \theta_{\mu}\right) b i n s} n_{i j}}{\sum_{j}^{\left(p_{\mu}, \theta_{\mu}\right) b i n s} n_{i j}^{\prime}} .
$$

The predictions for the default values $N_{j}$ and $n_{i j}$ are the same as the $N_{j}^{\text {pred }}$ and $n_{i j}^{\text {pred }}$ used in Eq. (6.1).

Unlike the flux and cross section uncertainties, intra-nuclear interaction (iii) and detector uncertainties (iv) are independent of the CC interaction rate. For these sources, the uncertainties are simply calculated as:

$$
N_{j}^{\prime}=\sum_{i}^{E_{\nu} b i n s} f_{i} n_{i j}^{\prime} .
$$

To generate the $\mathbf{V}_{\text {sys }}$ matrix, the systematically varied rate, $N_{i j}^{\prime}$, is compared to the default value of the event rate. To better estimate some uncertainties, many variation predictions ("draws") are used, each corresponding to a unique set of underlying parameters. To estimate the error from the $M_{A}^{Q E}$ uncertainty, for example, we generate 1000 different sets of MC expectations of $n_{i j}^{\prime}$, each corresponding to the different value of $M_{A}^{Q E}$ randomly drawn from the estimated uncertainty of $M_{A}^{Q E}$. Then, the error matrix representing the uncertainty from $M_{A}^{Q E}$ is calculated as:

$$
V_{j k}^{M_{A}^{Q E}}=\frac{1}{S} \sum_{l}^{S}\left(N_{j l}^{\prime}-N_{j}\right)\left(N_{k l}^{\prime}-N_{k}\right),
$$

where $k$ denotes the index of random draws and $S$ denotes the total number of draws. We estimate the errors from each source (i) - (iv) individually, and we add all matrices together to generate the total covariance matrix, $\mathbf{V}^{\text {sys }}$. We assume the same size of the fractional error for both NEUT and NUANCE based predictions, and the total covariance matrices for them are calculated by scaling the fractional errors with the predictions.

In this fit, we continuously update $\mathbf{V}^{\text {sys }}$ using the $f_{j}$ values. (i.e. The error matrix is recalculated at the every step in the fitting.)

\subsubsection{The size of systematic uncertainties}

Figure 6.5 shows the fractional size of the diagonal element $\left(\sqrt{V_{i i}} / N_{i}^{\text {pred }}\right)$ of the error at each $p_{\mu}$ vs. $\theta_{\mu}$ bin of SciBar-stopped and MRD-stopped samples. Figure 6.6 is the central values and its RMS of $\theta_{\mu}$ distribution from MRD-penetrated sample. The errors shown here are calculated with the all scale factors $\left(f_{j}\right)$ being set to 1 (before fit). 

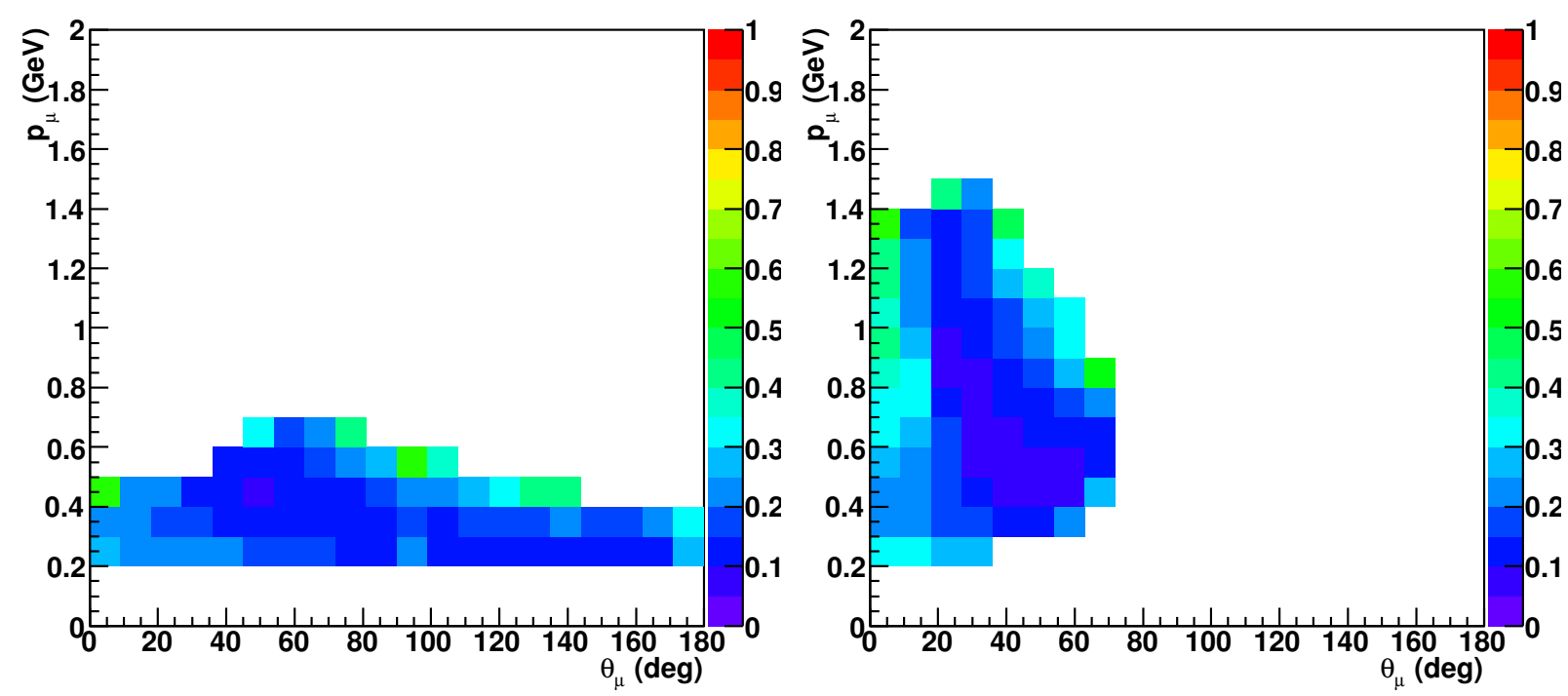

Figure 6.5: The fractional size of the diagonal element $\left(\sqrt{V_{i i}} / N_{i}^{\text {pred }}\right)$ of the systematic uncertainties at each $p_{\mu}$ vs. $\theta_{\mu}$ bin. The left plot shows the uncertainties for SciBar-stopped sample, and the right plot shows those for MRD-stopped sample.

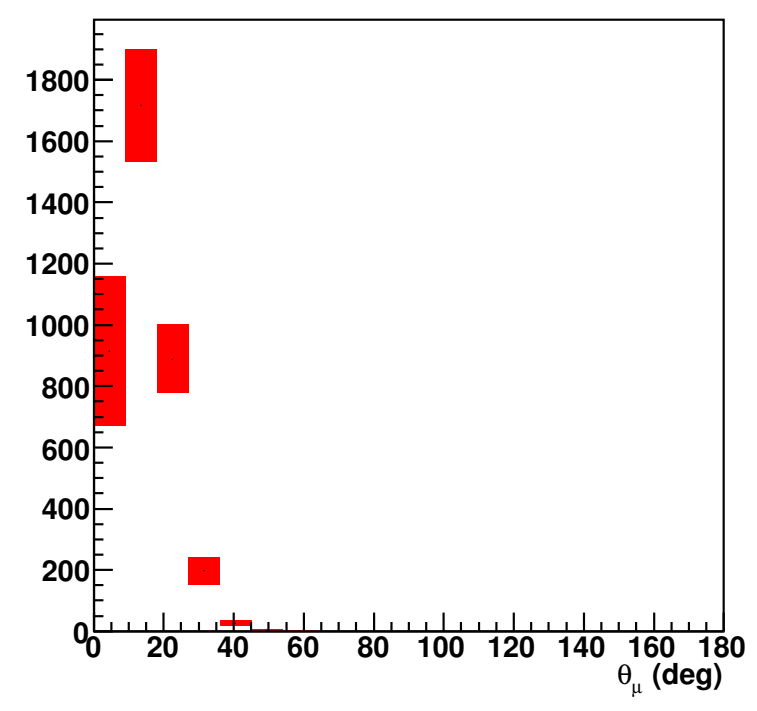

Figure 6.6: $\mathrm{MC}$ prediction and the total systematic uncertainties for the $\theta_{\mu}$ distribution of MRD-penetrated sample. The red filled region shows the size of the systematic uncertainty. 


\subsubsection{Spectrum fit result}

Tables 6.3 and 6.4 show the best fit parameters and their errors from the spectrum fit with the six energy dependent rate normalization factors for NEUT and NUANCE predictions, respectively. The $\chi^{2} / D O F$, where DOF is the number of degree of freedom for NEUT and NUANCE predictions are 161.2/153 and 173.6/153, respectively, while they are 225.1/159 and 560.7/159 before the fit. We obtain the reasonable $\chi^{2}$ values after the fit. We also tried to tune $Q^{2}$ distributions by fitting the parameters in the neutrino interaction models such as $M_{A}$ and $\kappa$, however, we do not find an improvement. So we decided to make fits just by using six rate normalization factors for true $E_{\nu}$ regions as described above, with the neutrino interaction parameters being fixed, because our method is less model dependent on interaction models.

Table 6.3: Spectrum fit result for the NEUT prediction. The first two rows are the best fit values and the uncertainties. The subsequent columns and rows represent the correlation coefficients for each parameters.

\begin{tabular}{c|cccccc}
\hline \hline & $f_{0}$ & $f_{1}$ & $f_{2}$ & $f_{3}$ & $f_{4}$ & $f_{5}$ \\
\hline Best fit & 1.042 & 1.032 & 1.234 & 1.290 & 1.193 & 0.789 \\
Error & 0.186 & 0.095 & 0.064 & 0.084 & 0.104 & 0.083 \\
\hline$f_{0}$ & 1.0000 & 0.2951 & 0.1653 & -0.2224 & -0.3646 & 0.0438 \\
$f_{1}$ & 0.2951 & 1.0000 & 0.0591 & -0.3674 & -0.5311 & -0.2187 \\
$f_{2}$ & 0.1653 & 0.0591 & 1.0000 & -0.2317 & -0.1511 & -0.3301 \\
$f_{3}$ & -0.2224 & -0.3674 & -0.2317 & 1.0000 & -0.0011 & 0.2534 \\
$f_{4}$ & -0.3646 & -0.5311 & -0.1511 & -0.0011 & 1.0000 & -0.0680 \\
$f_{5}$ & 0.0438 & -0.2187 & -0.3301 & 0.2534 & -0.0680 & 1.0000 \\
\hline \hline
\end{tabular}

Table 6.4: Spectrum fit result for the NUANCE prediction. The first two rows are the best fit values and the uncertainties. The subsequent columns and rows represent the correlation coefficients for each parameters.

\begin{tabular}{c|cccccc}
\hline \hline & $f_{0}$ & $f_{1}$ & $f_{2}$ & $f_{3}$ & $f_{4}$ & $f_{5}$ \\
\hline Best fit & 1.650 & 1.312 & 1.359 & 1.378 & 1.360 & 0.898 \\
Error & 0.203 & 0.093 & 0.057 & 0.075 & 0.107 & 0.085 \\
\hline$f_{0}$ & 1.0000 & 0.2260 & 0.0716 & -0.2470 & -0.2916 & 0.0019 \\
$f_{1}$ & 0.2260 & 1.0000 & 0.0244 & -0.4319 & -0.5275 & -0.2410 \\
$f_{2}$ & 0.0716 & 0.0244 & 1.0000 & -0.2225 & -0.1150 & -0.3403 \\
$f_{3}$ & -0.2470 & -0.4319 & -0.2225 & 1.0000 & 0.0910 & 0.2003 \\
$f_{4}$ & -0.2916 & -0.5275 & -0.1150 & 0.0910 & 1.0000 & -0.0569 \\
$f_{5}$ & 0.0019 & -0.2410 & -0.3403 & 0.2003 & -0.0569 & 1.0000 \\
\hline \hline
\end{tabular}

\subsubsection{Distributions after fit}

Figures 6.7 and 6.8 show the distributions of the difference between the observed and the predicted number of events, $\left(N_{j}^{\text {obs }}-N_{j}^{\text {pred }}\right) / N_{j}^{\text {pred }}$, in the $p_{\mu}-\theta_{\mu}$ planes for the SciBar-stopped and MRD-stopped samples, respectively. Distibutions for both before and after fits are shown in these figures. Figure 6.9 is the $\theta_{\mu}$ distribution for the MRD-penetraded sample. Finally, Figure 6.10 show the distributions of the difference divided by the total systematic and statistical errors, $\left(N_{j}^{\text {obs }}-N_{j}^{\text {pred }}\right) / N_{j}^{\text {pred }} / \sqrt{V_{j j}^{\text {sys }}+V_{j j}^{\text {stat }}}$. We find that the residual differences between the obeserved and the predicted number of events are covered by the systematic and statistical uncertainties, and the predictions of $p_{\mu}-\theta_{\mu}$ distributions after fit well reproduce the observed distributions. 

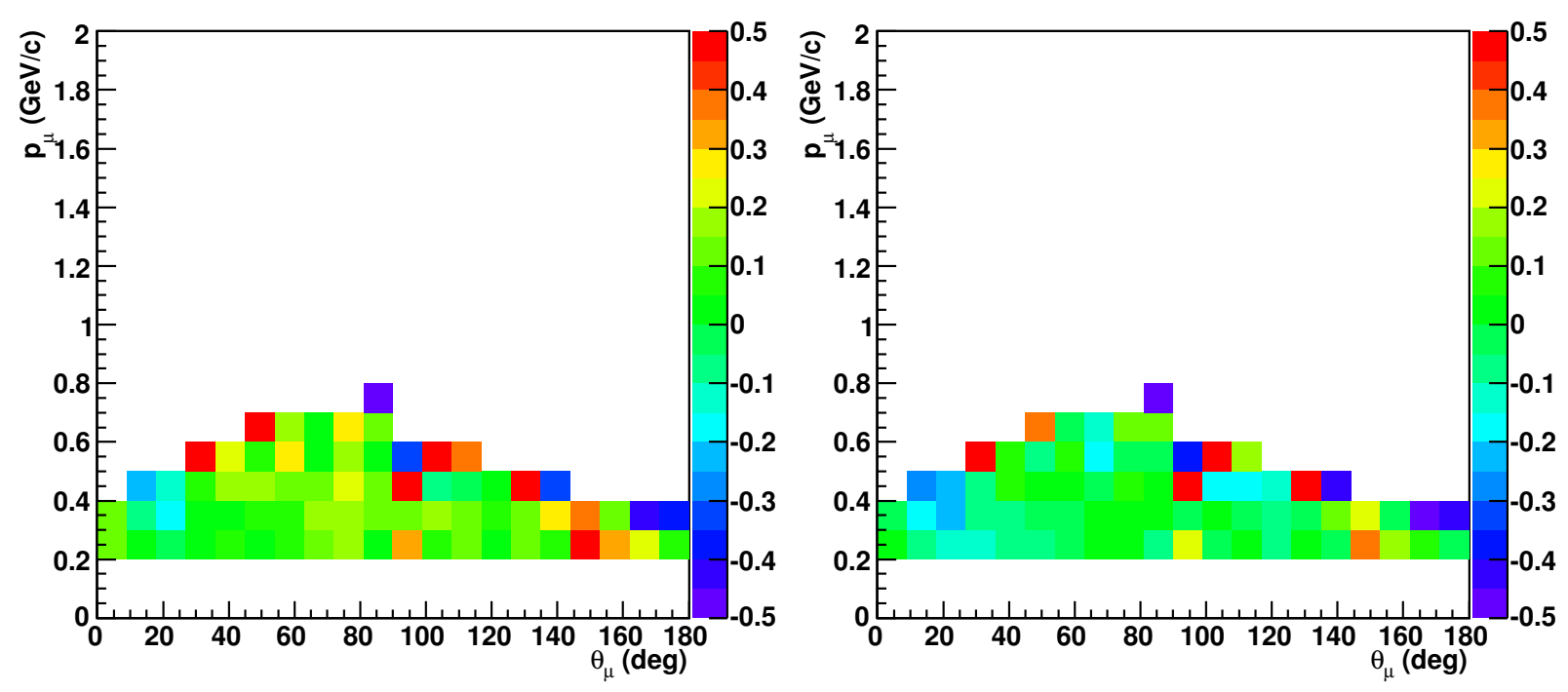

Figure 6.7: Distributions of the difference between the observed and the predicted numbers of events, $\left(N_{j}^{\text {obs }}-N_{j}^{\text {pred }}\right) / N_{j}^{\text {pred }}$, in the $p_{\mu}-\theta_{\mu}$ planes (left) before and (right) after fit for the SciBarstopped sample. Only bins used for the fit are shown in these plots.
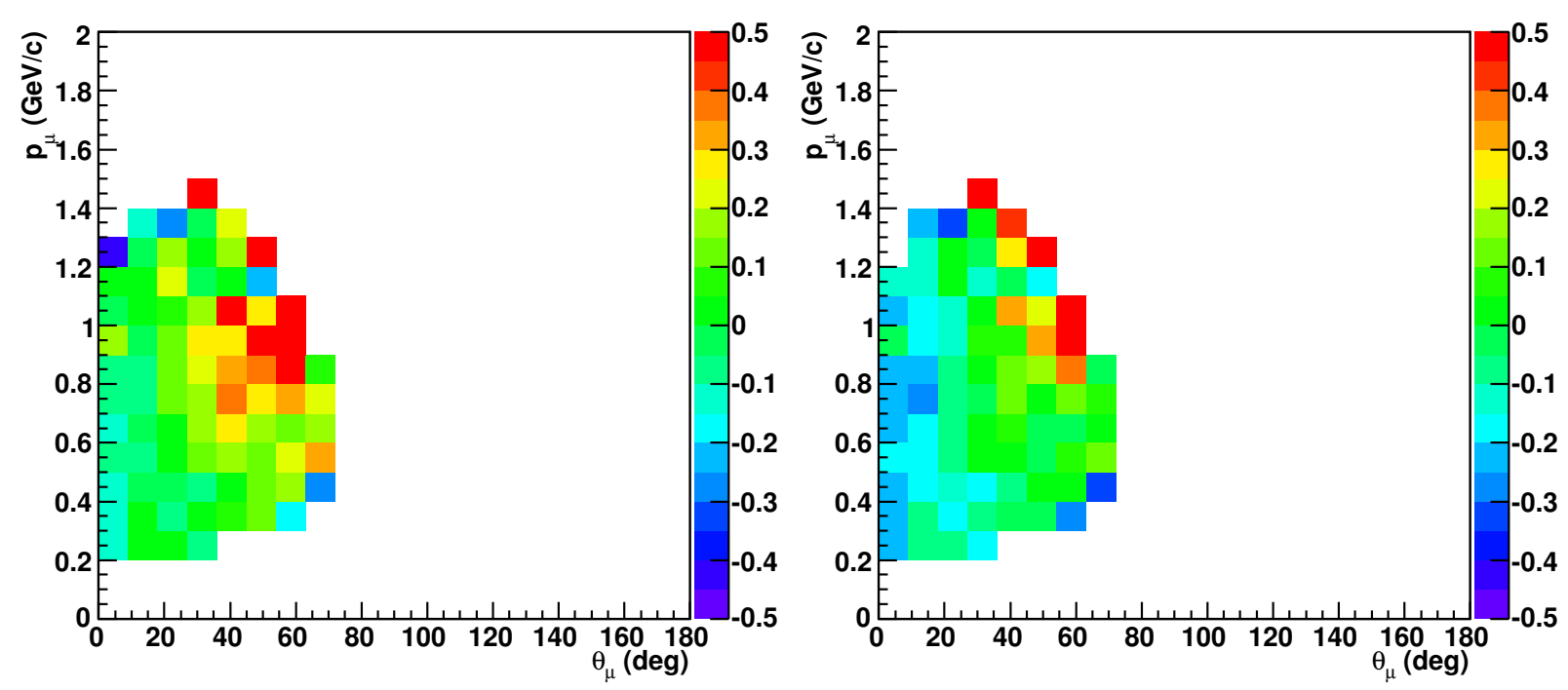

Figure 6.8: Distributions of the difference between the observed and the predicted numbers of events, $\left(N_{j}^{\text {obs }}-N_{j}^{\text {pred }}\right) / N_{j}^{\text {pred }}$, in the $p_{\mu}-\theta_{\mu}$ planes (left) before and (right) after fit for the MRDstopped sample. Only bins used for the fit are shown in these plots. 


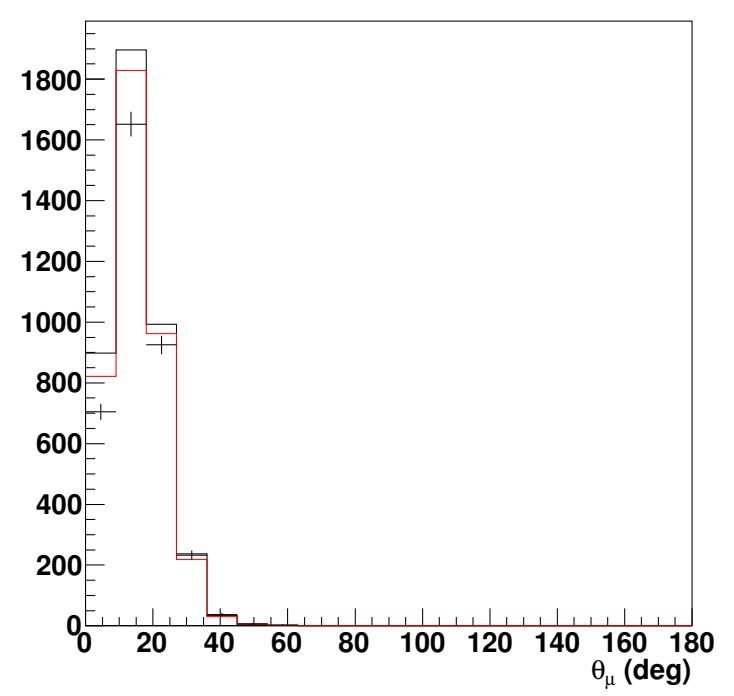

Figure 6.9: $\theta_{\mu}$ distribution for the MRDpenetrated sample. Black dots shows the data point. Black line is the MC prediction before fit, and the red line is the one after fit for the NEUT-based prediction.
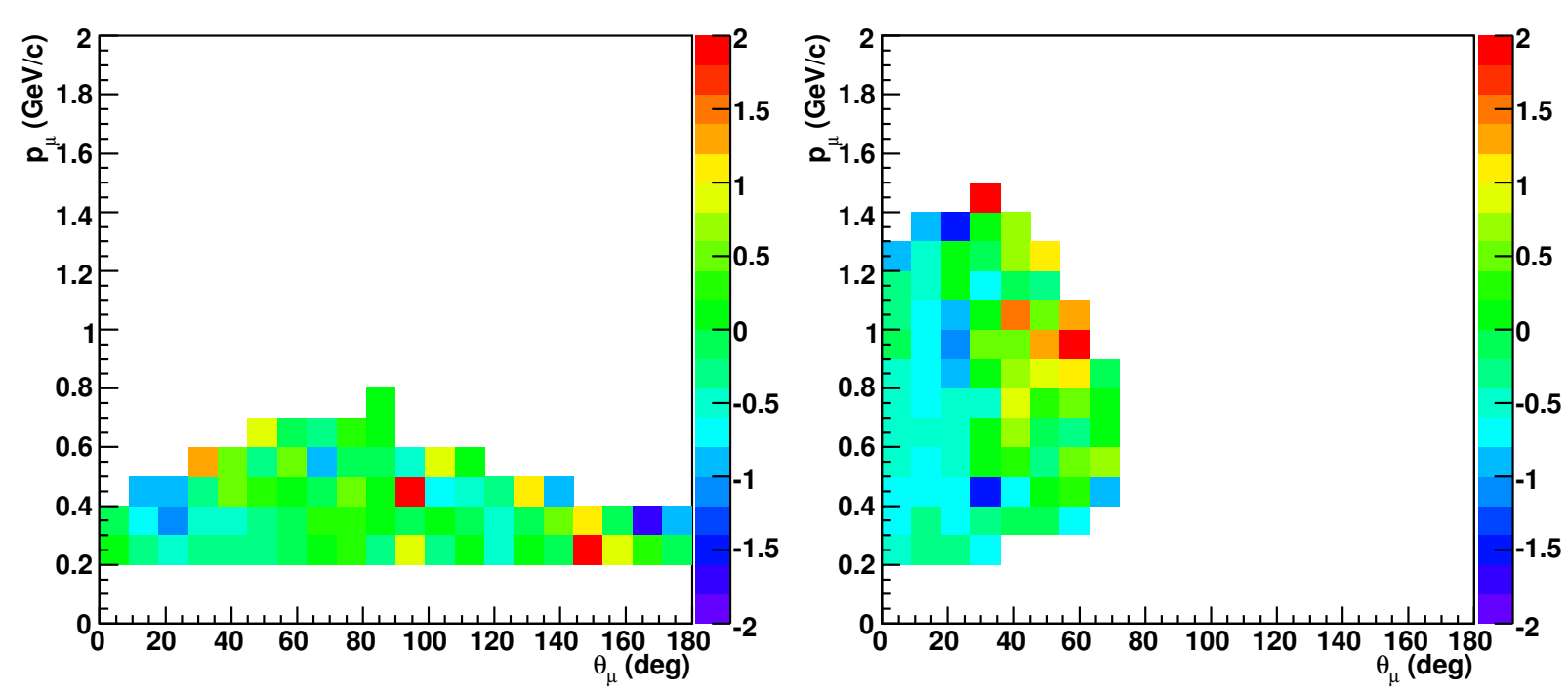

Figure 6.10: Distributions of the difference between the observed and predicted number of events, divided by the total errors, $\left(N_{j}^{\text {obs }}-N_{j}^{\text {pred }}\right) / N_{j}^{\text {pred }} / \sqrt{V_{j j}^{\text {sys }}+V_{j j}^{\text {stat }}}$ at each $P_{\mu}$ vs. $\theta_{\mu}$ bin. The left plot is that of SciBar-stopped sample, and the right one is that of MRD-stopped sample. Only bins used for the fit are shown in these plots. 
Figure 6.11 shows the distributions of $p_{\mu}, \theta_{\mu} E_{\nu}^{r e c}$ and $Q_{r e c}^{2}$ of the MRD-stopped sample, after applying the rate normalization factors obtained in this analysis. We reevaluate systematic error for each distribution in the same way as described in Sec. 6.2 .3 , where the systematic error is more constrained by this result. We also propagate the errors of the scale factors $\left(f_{i}\right)$ to the distributions. The errors on $f_{i}$ obtained from the fit include the shape error from all the flux and the cross section uncertainties, and the absolute error from all the intra-nuclear interaction and detector response uncertainties, as they are included into the error matrix $\left(V_{\text {sys }}\right)$. The errors shown in these plots are the quadrature sum of those systematic errors.

Similarly, Figure 6.12 shows the distributions of $p_{\mu}, \theta_{\mu} E_{\nu}^{r e c}$ and $Q_{r e c}^{2}$ of the SciBar-stopped sample, and Figure 6.13 shows the distribution of $\theta_{\mu}$ of the MRD-penetrated sample.

We find that both NEUT and NUANCE predictions well reproduce the data distributions within the errors obtained in this analysis. Also, we confirm that this measurement can reduce the systematic uncertainty of the flux and the cross section in most regions, compared to the original errors.
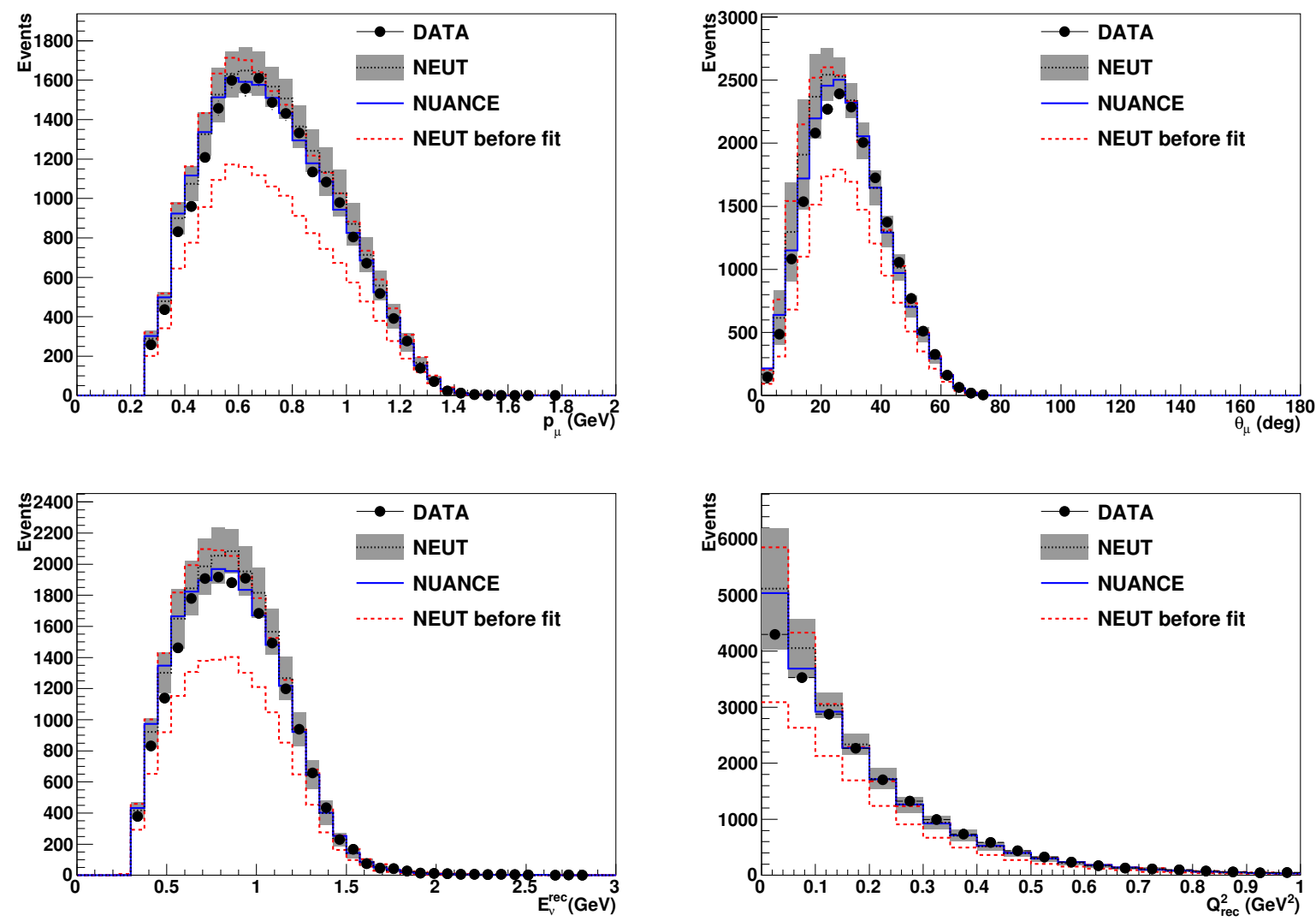

Figure 6.11: Top: Reconstructed $p_{\mu}$ (left) and $\theta_{\mu}$ (right) distributions for the MRD-stopped sample. Bottom: $E_{\nu}^{r e c}$ (left) and $Q_{r e c}^{2}$ (right) distributions for the MRD-stopped sample. The results of the spectrum fit are applied for the NEUT and NUANCE predictions, with the normalization by the number of POT. The filled regions show the systematic uncertainties of MC predictions based on NEUT. The area within dashed lines shows the NEUT predictions with their systematic uncertainties before the fit. The systematic uncertainty for the NUANCE prediction is similar to that of the NEUT prediction and is not shown. 

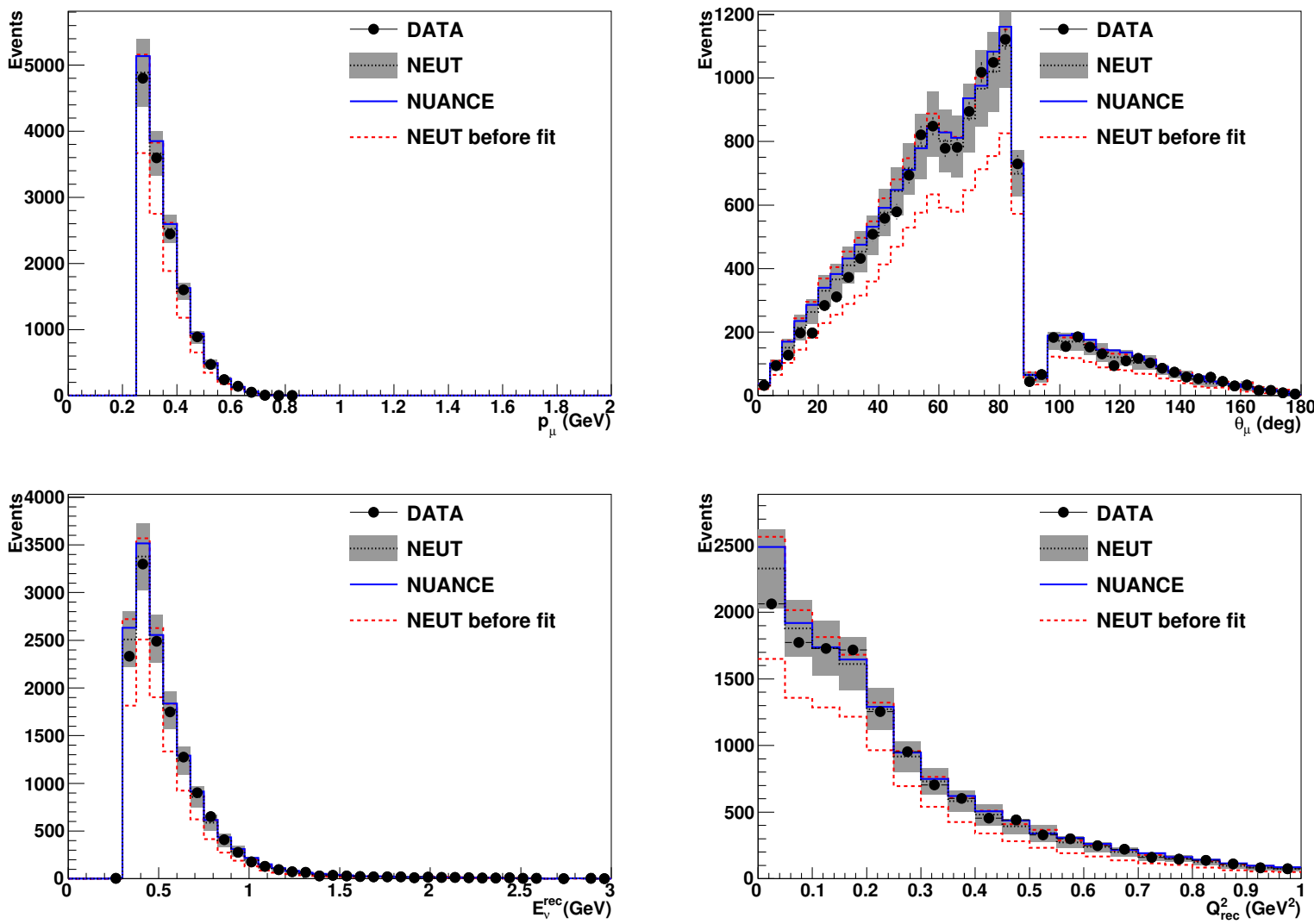

Figure 6.12: Top: Reconstructed $p_{\mu}$ (left) and $\theta_{\mu}$ (right) distributions for the SciBar-stopped sample. Bottom: $E_{\nu}^{r e c}$ (left) and $Q_{r e c}^{2}$ (right) distributions for the SciBar-stopped sample. The results of the spectrum fit are applied for the NEUT and NUANCE predictions, with the normalization by the number of POT. The filled regions show the systematic uncertainties of MC predictions based on NEUT. The area within dashed lines shows the NEUT predictions with their systematic uncertainties before the fit. The systematic uncertainty for the NUANCE prediction is similar to that of the NEUT prediction and is not shown.

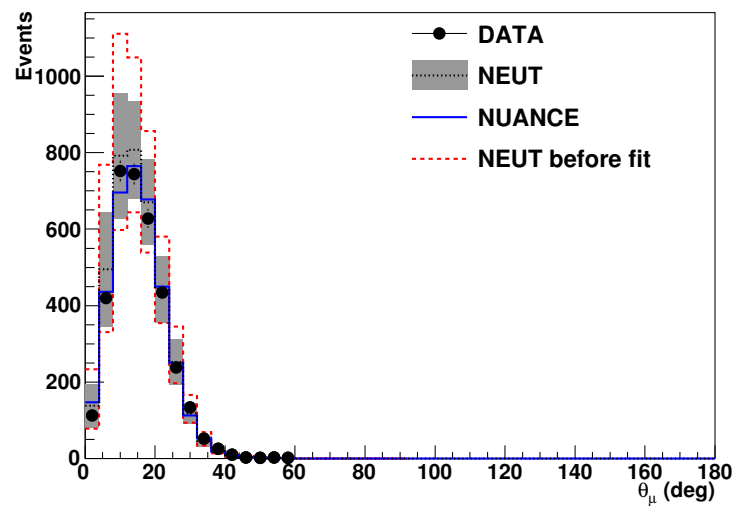

Figure 6.13: Reconstructed $\theta_{\mu}$ distribution for the MRD-penetrated sample. The results of the spectrum fit are applied for the NEUT and NUANCE predictions, with the normalization by the number of POT. The filled regions show the systematic uncertainties of MC predictions based on NEUT. The area within dashed lines shows the NEUT predictions with their systematic uncertainties before the fit. The systematic uncertainty for the NUANCE prediction is similar to that of the NEUT prediction and is not shown. 


\subsection{Results and discussions}

In this section, we report on the extraction of the $\mathrm{CC}$ interaction rates and cross sections. As described in Sec. 2.2, the rate measurement is primary motivated by the SciBooNE-MiniBooNE joint $\nu_{\mu}$ disappearance analysis, while the cross section results can be used to test various neutrino interaction models. In addition, we also extract flux integrated rates and cross sections as a cross check of the energy dependent analysis. These results for energy dependent and flux intergrated analyses are given in Sec. 6.3.1 and Sec. 6.3.2, respectively. Then, we discuss the interpretation of the results in Sec. 6.3.3.

\subsubsection{Energy dependent $\mathrm{CC}$ interaction rate and cross section}

\subsubsection{CC interaction rate extraction}

The CC interaction rate in i-th true $E_{\nu}$ region, $\mathcal{R}_{i}$, is calculated with the obtained $f_{i}$ as:

$$
\mathcal{R}_{i}=\frac{f_{i} \cdot \mathcal{N}_{i}^{\text {pred }} \cdot P_{i}}{\epsilon_{i}}
$$

where $\mathcal{N}_{i}^{\text {pred }}$ is the number of selected events predicted by the MC simulation, $P_{i}$ is the purity of the $\mathrm{CC}$ inclusive sample, and $\epsilon_{i}$ is the efficiency. We evaluate the errors on $P_{i} / \epsilon_{i}$ with all systematic uncertainties listed in Table 6.2. Then, we take the quadrature sum of the errors of $f_{i}$ and $P_{i} / \epsilon_{i}$ to estimate the total systematic error. Figure 6.14 shows the obtained neutrino interaction rate normalized to the NEUT and NUANCE predictions, with the full systematic error. The original flux and cross-section uncertainties are also shown in the plot. The numerical values of the interaction rate normalized to the MC predictions and its full errors are shown in Table 6.5. The uncertainty is about $6 \%$ for the interaction rate at $0.75<E_{\nu}<1.0 \mathrm{GeV}$, where the CC interaction rate is maximum. The uncertainty is as large as $15 \%$ in the lowest energy region.

Table 6.5: $\nu_{\mu}$ CC inclusive interaction rate normalization factors to NEUT and NUANCE predictions. The size of the full systematic errors are also shown.

\begin{tabular}{ccc}
\hline \hline $\begin{array}{c}\text { Energy region } \\
(\mathrm{GeV})\end{array}$ & \multicolumn{2}{c}{$\nu_{\mu}$ CC rate normalization factor } \\
NEUT & NUANCE \\
\hline $0.25-0.50$ & $1.04 \pm 0.20$ & $1.65 \pm 0.22$ \\
$0.50-0.75$ & $1.03 \pm 0.11$ & $1.31 \pm 0.11$ \\
$0.75-1.00$ & $1.23 \pm 0.08$ & $1.36 \pm 0.08$ \\
$1.00-1.25$ & $1.29 \pm 0.10$ & $1.38 \pm 0.09$ \\
$1.25-1.75$ & $1.19 \pm 0.11$ & $1.36 \pm 0.12$ \\
$1.75-$ & $0.79 \pm 0.08$ & $0.90 \pm 0.09$ \\
\hline \hline
\end{tabular}

\subsubsection{CC inclusive cross section}

The CC inclusive cross section per nucleon on polystyrene target $\left(\mathrm{C}_{8} \mathrm{H}_{8}\right)$ at each energy region is calculated as

$$
\sigma_{i}=f_{i} \cdot\left\langle\sigma_{C C}^{\text {pred }}>_{i}=\frac{f_{i} \cdot \mathcal{N}_{i}^{\text {pred }} \cdot P_{i}}{\epsilon_{i} \cdot T \cdot \Phi_{i}}\right.
$$

where $i$ is the index of the energy regions used for the spectrum fit (see Table 6.1 ,,$\left\langle\sigma_{C C}^{\text {pred }}>_{i}\right.$ is the predicted flux averaged CC interaction cross section per nucleon, $\mathcal{N}_{i}^{\text {pred }}$ is the number of selected events predicted by the MC simulation, $P_{i}$ is the purity of $\mathrm{CC}$ inclusive sample, $\epsilon_{i}$ is the efficiency, $T_{i}$ is the number of nucleons in the SciBar fiducial volume, and $\Phi_{i}$ is the muon neutrino flux per unit area. 


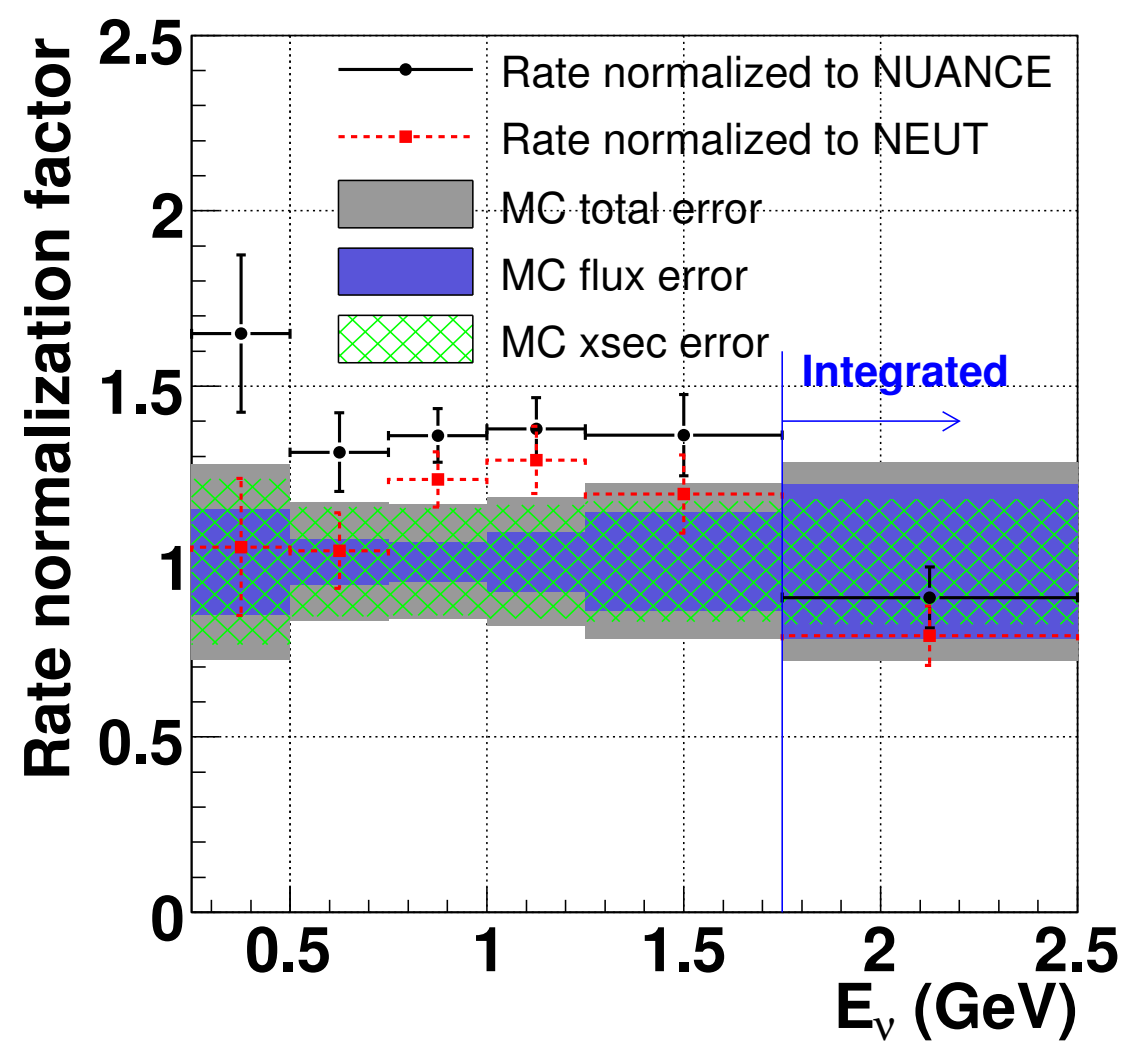

Figure 6.14: The CC interaction rates normalized to the NEUT and NUANCE predictions, obtained by the spectrum fit. The error bars show the full systematic uncertainties. The original errors of the flux and cross section uncertainties are also shown separately. 
Figure 6.15 show the extracted cross sections plotted with the original predictions by NEUT and NUANCE. In addition to the errors on $P_{i} / \epsilon_{i}$ as estimated for the rate measurements, we also estimate the errors on $\Phi_{i}$ from the category (i) in the table. In the plot, we separately show the errors of $f_{i}$ and the quadrature sum of $f_{i}, P_{i}, \epsilon_{i}$ and $\Phi_{i}$ errors.

The obtained cross section values and their errors are summarized in Table 6.6. The uncertainty on the cross section is about $10 \%$ at $0.75<E_{\nu}<1.0 \mathrm{GeV}$, where the CC interaction rate is maximum. The uncertainty is as large as 30\% in the lowest energy region. This is the first measurement of CC inclusive interaction cross section on carbon around $1 \mathrm{GeV}$.

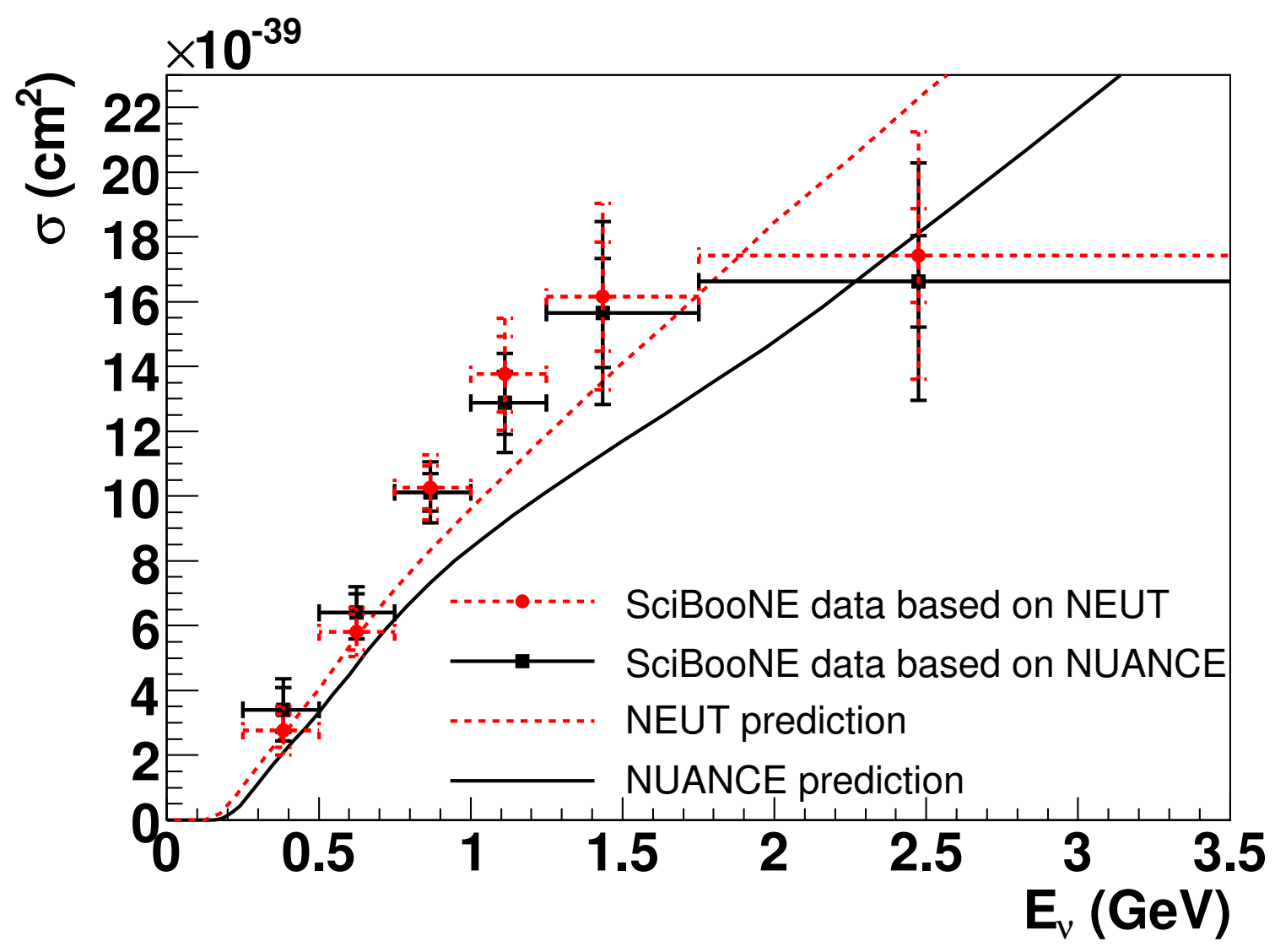

Figure 6.15: $\mathrm{CC}$ inclusive interaction cross section per nucleon on a polystyrene target $\left(\mathrm{C}_{8} \mathrm{H}_{8}\right)$. The smaller error bars show the uncertainties of the rate normalization factors, and the larger error bars represents the total error including the flux uncertainties.

\subsubsection{Flux integrated total CC interaction rate and cross section}

\subsubsection{Measurement of $\mathrm{CC}$ interaction rate}

We perform a fit with the method described in Sec. 6.2 using one rate normalization factor $\left(f_{\text {tot }}\right)$ which spans the entire energy region, instead of six regions. This flux integrated measurement is motivated as a cross check of the energy dependent analysis.

The obtained rate normalization factors are $1.12 \pm 0.02 \pm 0.03$, and $1.29 \pm 0.02 \pm 0.03$ for the NEUT and NUANCE predictions, respectively. The first errors show the errors from the fit, and the second show the errors from the uncertainties of efficiency and purity. The $\chi^{2} / D O F$, where DOF is the number of degree of freedom for NEUT and NUANCE predictions are 173.0/158 and 183.0/158, respectively, while they are 208.6/159 and 481.5/159 before the fit.

In this measurement, we obtained the higher rate normalization factors compared to the simple ratio of the number of events (Table 5.4). This is because the "shape" systematic uncertainty 
Table 6.6: Energy dependent $\mathrm{CC}$ inclusive cross section per nucleon on a polystyrene target $\left(\mathrm{C}_{8} \mathrm{H}_{8}\right)$. Results based on NEUT and NUANCE based predictions are separately shown.

\begin{tabular}{ccccc}
\hline \hline $\begin{array}{c}\text { Energy region } \\
(\mathrm{GeV})\end{array}$ & $\begin{array}{c}\text { Mean Energy } \\
(\mathrm{GeV})\end{array}$ & $\begin{array}{c}\text { Total } \nu_{\mu} \text { flux } \\
\left(\nu_{\mu} / \mathrm{cm}^{2}\right)\end{array}$ & \multicolumn{2}{c}{$\nu_{\mu}$ CC inclusive cross section $\left(\mathrm{cm}^{2} / \mathrm{nucleon}^{2}\right)$} \\
NEUT based & NUANCE based \\
\hline $0.25-0.50$ & 0.38 & $(4.31 \pm 0.81) \times 10^{11}$ & $(2.76 \pm 0.75) \times 10^{-39}$ & $(3.40 \pm 0.96) \times 10^{-39}$ \\
$0.50-0.75$ & 0.62 & $(5.09 \pm 0.37) \times 10^{11}$ & $(5.80 \pm 0.75) \times 10^{-39}$ & $(6.39 \pm 0.81) \times 10^{-39}$ \\
$0.75-1.00$ & 0.87 & $(4.18 \pm 0.26) \times 10^{11}$ & $(1.03 \pm 0.10) \times 10^{-38}$ & $(1.01 \pm 0.09) \times 10^{-38}$ \\
$1.00-1.25$ & 1.11 & $(2.63 \pm 0.23) \times 10^{11}$ & $(1.38 \pm 0.17) \times 10^{-38}$ & $(1.29 \pm 0.15) \times 10^{-38}$ \\
$1.25-1.75$ & 1.43 & $(1.90 \pm 0.27) \times 10^{11}$ & $(1.62 \pm 0.29) \times 10^{-38}$ & $(1.56 \pm 0.28) \times 10^{-38}$ \\
$1.75-$ & 2.47 & $(0.62 \pm 0.12) \times 10^{11}$ & $(1.74 \pm 0.38) \times 10^{-38}$ & $(1.66 \pm 0.37) \times 10^{-38}$ \\
\hline \hline
\end{tabular}

(Sec. 6.2.3) is calculated over the entire energy region, and it typically becomes smallest where the $\mathrm{CC}$ interaction rate is maximum. Hence, the obtained rate normalization factors tend to be close to the results for $0.75<E_{\nu}<1.0 \mathrm{GeV}$ in the energy dependent analysis (Sec. 6.3.1).

\subsubsection{CC inclusive cross section}

We extract the total $\mathrm{CC}$ inclusive cross section for the flux at $E_{\nu}>0.25 \mathrm{GeV}$ using a similar formula to Eq. (6.9):

$$
\sigma=f_{t o t} \cdot\left\langle\sigma_{C C}^{\text {pred }}\right\rangle=\frac{f_{\text {tot }} \cdot \mathcal{N}^{\text {pred }} \cdot P}{\epsilon \cdot T \cdot \Phi}
$$

The purity $(P)$ and the efficiency $(\epsilon)$ are estimated for the sum of SciBar-stopped, MRD-stopped and MRD-penetrated samples, and obtained to be $P=90.0(89.1) \%$ and $\epsilon=34.5(34.5) \%$ with the NEUT(NUANCE) generators. The integrated $\nu_{\mu}$ flux at $E_{\nu}>0.25 \mathrm{GeV}$ averaged over the SciBar Fiducial Volume for 9.9E19 POT is estimated to be $\Phi=(1.87 \pm 0.14) \times 10^{12}\left(\nu_{\mu} / \mathrm{cm}^{2}\right)$, with mean energy of $0.83 \mathrm{GeV}$.

Using these purity, efficiency and flux predictions, we obtain the total CC cross sections per nucleon on a polystyrene target to be $(8.51 \pm 0.72) \times 10^{-39}$ and $(8.45 \pm 0.71) \times 10^{-39}$ for NEUT and NUANCE based predictions, respectively. The error contains all systematic uncertainties evaluated in this analysis. The error is dominated by the uncertainty on the total flux prediction $(7.6 \%)$.

\subsubsection{Discussion of the CC rate and cross section measurement results}

This analalyis is the first measurement of the $\mathrm{CC}$ inclusive interaction rate and cross section on carbon in the $1 \mathrm{GeV}$ region. This measurement is also unique in the sence that the results covers a wide energy range from $0.25 \mathrm{GeV}$ to $\sim 3 \mathrm{GeV}$ by combining several sub-samples in SciBooNE. Hence, this result provides an useful input to tune the neutrino interaction models and/or the neutrino flux model, which helps current and future neutrino oscillation experiments conducted in this energy region, such as MiniBooNE, $\mathrm{T} 2 \mathrm{~K}$ and $\mathrm{NO} \nu \mathrm{A}$.

\subsubsection{Consistency between NEUT and NUANCE}

As described before, we find a large difference of the rate normalization factors between NEUT and NUACNE based simulations. The major source of the rate normalization factors difference is (a) the difference in the total neutrino interaction cross sections of the two models. This effect is compensated by the rate normalization factor obtained in this analysis, and thus has little effect on the absolute rates.

However, there is also a second-order contribution to this difference from (b) the difference in the prediction of the final state particle production and the kinematics. This affects events with a 
pion or a proton tracks mis-reconstructed as a muon, which ultimately affects the purity $\left(P_{i}\right)$ and the efficiency $\left(\epsilon_{i}\right)$ in Eq. 6.8).

On the other hand, for the extracted cross sections, the difference between NEUT and NUANCE due to (a) is canceled, and the remaining differences are, in principle, due to the source (b). Hence, we can estimate the effect of (b). From Figure 6.15, we confirm that the differences of the extracted CC interaction cross sections between NEUT and NUANCE are within the errors of $f_{i}$. Therefore, the effect of source (b) is small and covered by the systematic uncertainty. The difference of the rate normalization factors is mostly caused by the cross section difference itself (source (a)).

As a next step, we can test various neutrino interaction models by implementing these into NEUT and NUANCE, and see if there is a model consistently describe the observed data well. This will be a crucial step for the next high precision neutrino oscillation measurements.

\subsubsection{Comparison with other CC measurements}

Figure 6.16 show the obtained cross sections from both energy dependent and flux integrated analyses, compared with the results from BNL-7ft bubble chamber with deuterium target 53 . (around $1 \mathrm{GeV}$ ), NOMAD [50] and MINOS [51] (above $\sim 2 \mathrm{GeV}$ ). The results are consistent with these previous measurements. The dominant error of our cross section result is due to the neutrino flux uncertainy. We should note that, in the BNL bubble chamber measurement, they use CC-QE interaction to normalize the absolute neutrino flux. Hence their result depends on the CC-QE cross section models. On the other hand, our flux uncertainty is estimated by the uncertainty of the hadron production cross sections from proton-Be interactions. Therefore, our result is less model dependent and reliable, although our error is larger than the previous measurement at BNL.

An interesting topic in this $1 \mathrm{GeV}$ regain is the recent measurement of CC-QE interaction cross sections on carbon at $0.4<E_{\nu}<2.0(\mathrm{GeV})$ by MiniBooNE [66], as shown in Figure 1.8. They found that the obtained cross section is higher than what extrapolated from a measurement at $E_{\nu}>3(\mathrm{GeV})$ by NOMAD [67]. This initiates a large number of discussions on the CC-QE cross section models. The MiniBooNE CC-QE sample has $\sim 20 \%$ contamination of CC- $1 \pi$ backgrounds, and the size of this contamination have large uncertainty because of the uncertainty of the pion final state interaction (FSI) in nucleus. So this discrepancy could be due to mis-estimation of the background. Hence, CC inclusive cross section measurement, which is not affected by the FSI uncertainty, have been awaited. Our result covers not only the $E_{\nu}$ region for MiniBooNE, but also the intermediate region at $2<E<3(\mathrm{GeV})$, and therefore is complementary to these CC-QE measurements. Figure 6.17 shows the CC-inclusive interaction cross section based on the NUANCE prediction, compared with the CC-QE cross section measured by MiniBooNE [66]. Interestingly, our result also indicates the higher cross sections than the prediction around $1 \mathrm{GeV}$, while the cross section at the highest energy region $\left(E_{\nu}>1.75 \mathrm{GeV}\right)$ is consistent to the prediction. There are also CC- $1 \pi$ exclusive cross section measurements forthcoming from SciBooNE and MiniBooNE. Further analyses comparing these exclusive measurements with our inclusive measurement will reveal the effect of the FSI. Then, a precise determination of the CC interaction cross section in the few-GeV region become possible.

\subsection{Applications to other measurements}

As mentioned before, this CC interaction analysis is also motivated by the two direct applications to the measurements at SciBooNE and MiniBooNE; providing absolute normalizations of previous SciBooNE's cross section results, and providing constraints of the flux and the cross section for a neutrino oscillation analysis at MiniBooNE. We discuss these two applications in this section. 


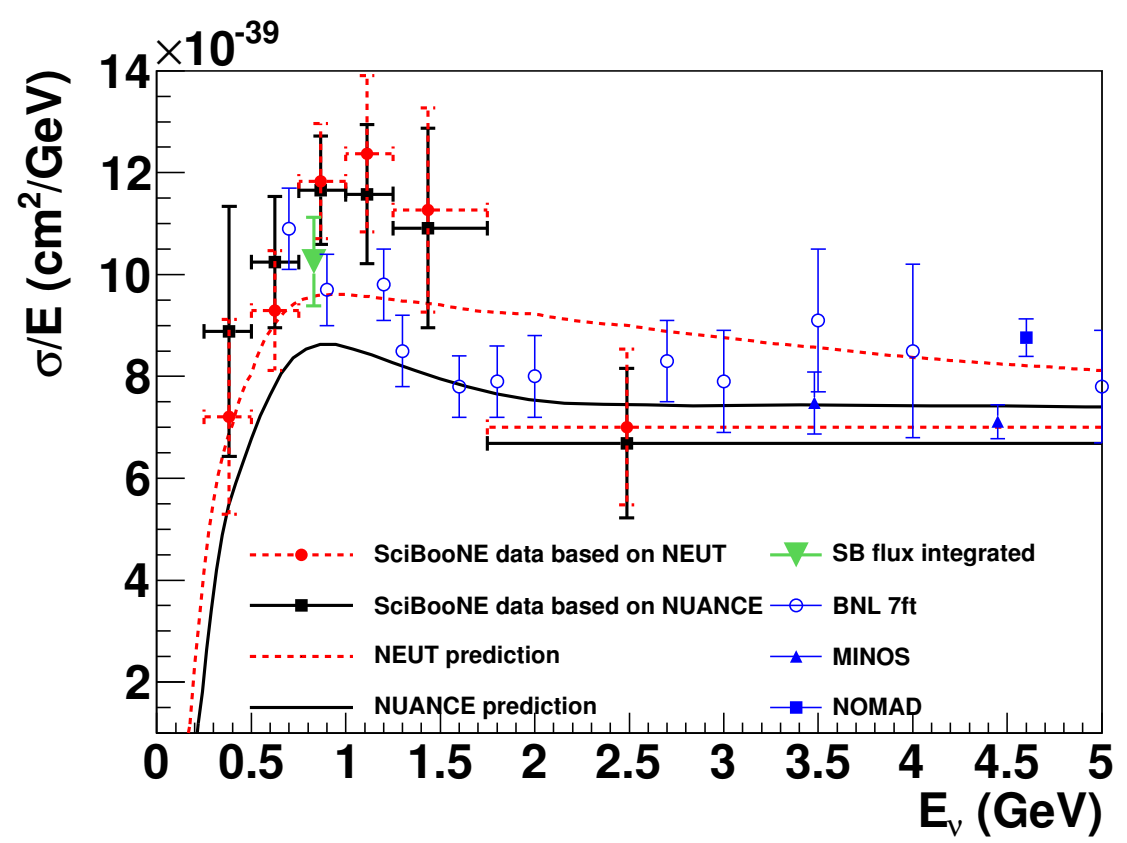

Figure 6.16: Extracted CC inclusive interaction cross sections, compared to the previous measurements. Results from BNL-7ft bubble chamber [53] NOMAD [50] and MINOS [51] are shown. For the SciBooNE's flux integrated cross-section, the result based on NEUT is shown.

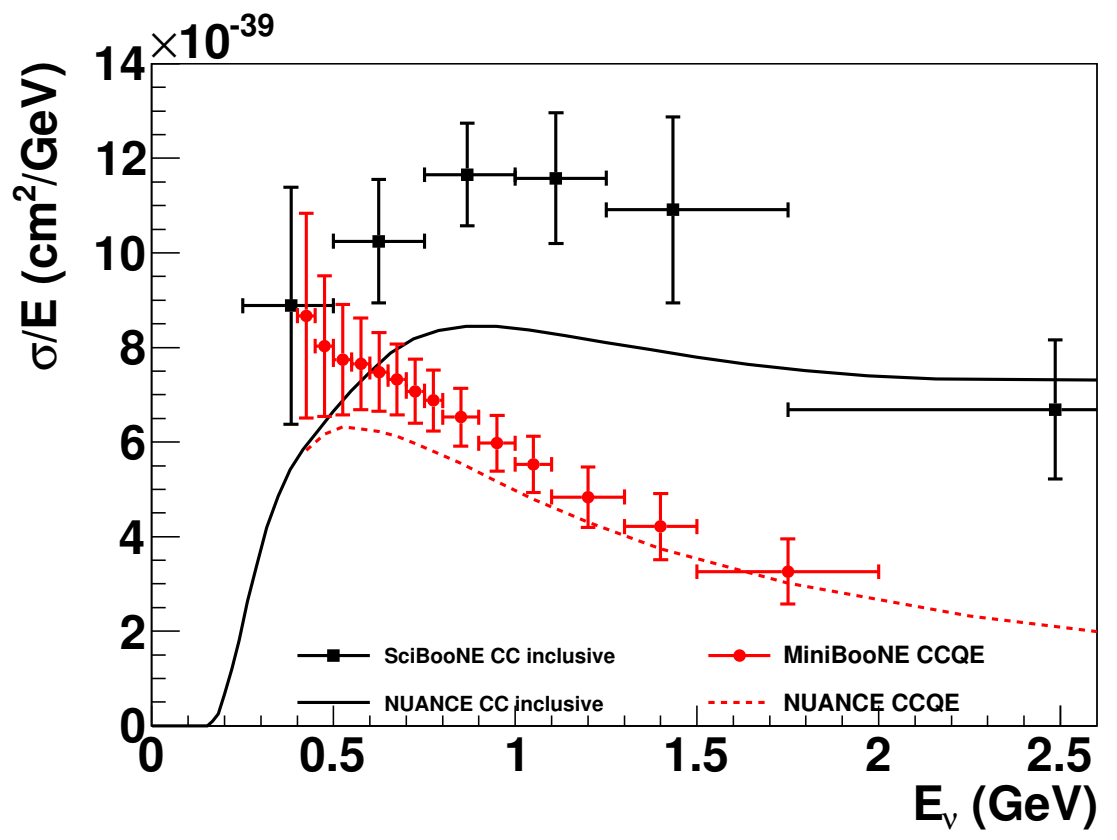

Figure 6.17: Extracted NUANCE-based CC inclusive interaction cross sections, compared with the CC-QE cross section measured by MiniBooNE [66]. The original NUANCE predictions are also shown. 


\subsubsection{Absolute normalization for previous SciBooNE measurements}

Previously, SciBooNE collaboration published cross section ratio measurements for charged current coherent pion production [68] and neutral current neutral pion production [69, 70]. These are especially important for the neutrino oscillation experiments in the $1 \mathrm{GeV}$ region, since the CC pion production and $\mathrm{NC}$ pion production are the the largest backgrounds for $\nu_{\mu}$ disappearance and $\nu_{e}$ appearance measurements, respectively.

For these measurements, the total CC interaction cross sections were used to normalize the results. However, these results are usually hard to compare with the theoretical models since the models usually predict the absolute cross sections, not the ratios to the total CC interaction cross section.

When we published the cross section results at SciBooNE, we did not have the CC cross section measurements because we did not have the reliable flux uncertainties; it is first estimated for this analysis. Then, we also provide the values of the flux integrated CC interaction cross section with the definitions used for the previous publications in the following part.

We have used the number of MRD-matched and MRD-penetrated samples to extract the total $\mathrm{CC}$ cross section for $\mathrm{CC}$ coherent pion production measurements [68, and used MRD-stopped sample for $\mathrm{NC}$ neutral pion production measurements [69, 70]. We extract the absolute CC inclusive cross section from the number of each sub-sample as:

$$
\sigma=\frac{\mathcal{N}^{o b s} \cdot P}{\epsilon \cdot T \cdot \Phi}
$$

where $\mathcal{N}^{\text {obs }}$ is the observed number of events in each sub-sample, $P$ is the purity of $\nu_{\mu}$ CC interaction in the sample. Table 6.7 is a summary of the absolute CC cross section extracted from the number of events in each sub-sample.

Table 6.7: Extracted number of total CC interaction and absolute cross sections per nucleon on a polystyrene target, based on the number of events in each sub-sample. The MC predictions are based on NEUT.

\begin{tabular}{cccccc}
\hline \hline sample & $\begin{array}{c}\text { Events } \\
\left(\mathcal{N}^{\text {obs }}\right)\end{array}$ & $\begin{array}{c}\text { Efficiency } \\
(\epsilon)\end{array}$ & $\begin{array}{c}\text { Purity } \\
(P)\end{array}$ & $\begin{array}{c}\text { Total CC events } \\
\left(\mathcal{N}^{\text {obs }} P / \epsilon\right)\end{array}$ & $\begin{array}{c}\text { CC cross-section } \\
\left(\sigma\left[\mathrm{cm}^{2} / \text { nucleon }\right]\right)\end{array}$ \\
\hline MRD-match & 28409.6 & $27.2 \%$ & $92.9 \%$ & $(9.71 \pm 0.69) \times 10^{4}$ & $(8.14 \pm 0.85) \times 10^{-39}$ \\
MRD-stop & 20236.4 & $18.5 \%$ & $91.2 \%$ & $(10.0 \pm 0.73) \times 10^{4}$ & $(8.34 \pm 0.88) \times 10^{-39}$ \\
MRD-pene & 3544.4 & $4.3 \%$ & $97.1 \%$ & $(7.94 \pm 1.54) \times 10^{4}$ & $(6.65 \pm 1.29) \times 10^{-39}$ \\
\hline \hline
\end{tabular}

Since we made minor improvements to the MC prediction and the reconstruction algorithms, the number of events in each sub-sample are slightly different from the previous published results. The difference of the number of events are shown in Table 6.7, and are accounted as the systematic uncertainties.

Table 6.8: The difference of total number of CC events, between this and previous works.

\begin{tabular}{|c|c|c|c|}
\hline \multicolumn{4}{|c|}{ Total CC events } \\
\hline Base ample & This result & Previous result & Difference \\
\hline MRD-match & $0.971 \times 10^{5}$ & $1.009 \times 10^{5} \quad 68$ & $3.2 \%$ \\
\hline MRD-stop & $1.000 \times 10^{5}$ & $1.019 \times 10^{5} \quad 69,70$ & $1.9 \%$ \\
\hline MRD-pene & $0.794 \times 10^{5}$ & $0.804 \times 10^{5} 68$ & $0.5 \%$ \\
\hline
\end{tabular}

For the cross section values extracted from the MRD-matched and the MRD-stopped samples, the dominant source of uncertainty is the total flux $(\Phi)$ uncertainty $(7.6 \%)$. The second largest 
uncertainty is the efficiency $(\epsilon)$ uncertainty due to the cross section models. They are $4.0 \%$ and $5.0 \%$ in the MRD-matched and the MRD-stopped samples, respectively.

For the MRD-penetrated sample, the dominant systematic error from the efficiency variation is due to the flux model uncertainties. Approximately $40 \%$ of the MRD-penetrated sample is from neutrinos from kaon decay, which have a large uncertainty, as shown in Fig. 4.2. The error from the efficiency variation due to the flux model is estimated to be $18.1 \%$. The second largest error comes from the the total flux $(\Phi)$ uncertainty $(7.6 \%)$.

\subsubsection{SciBooNE-MiniBooNE joint $\nu_{\mu}$ disappearance analysis}

As discussed in Sec. 1.4, we search for $\nu_{\mu}$ disappearance at $\Delta m^{2} \sim 1 \mathrm{eV}^{2}$ to test the LSND signal. Since the previous MiniBooNE-only measurement is limited by the large flux and cross section uncertainties, we improve the $\nu_{\mu}$ disappearance analysis by using this CC interaction measurement at SciBooNE.

For this analysis, the result of the CC interaction rate measurement and techniques for the systematic error analysis described in this chapter are directly used. We will describe this SciBooNEMiniBooNE joint $\nu_{\mu}$ disappearance analysis in Chapter 7 .

\subsection{Summary}

In Summary, we presented the measurements of muon neutrino $\mathrm{CC}$ interaction rate and cross section at SciBooNE.

We extract the $\nu_{\mu} \mathrm{CC}$ interaction rates by fitting the muon kinematics, with precision of 6$15 \%$ for the energy dependent and 3\% for the energy integrated analyses. We confirm that the distributions after fitting well reproduce the observed distributions with both NEUT and NUANCE based simulations. We also evaluate the CC inclusive interaction cross sections, and the results are consistent with both NEUT and NUANCE predictions. This confirms that the difference in the observed rates normalized to the NEUT and NUANCE based predictions is mainly due to the cross section difference of the two simulators. The precisions of the obtained cross sections are 10-30\% for energy dependent and $8 \%$ for the energy integrated analyses. This is the first measurement of the $\mathrm{CC}$ inclusive cross section on carbon around $1 \mathrm{GeV}$. Also this measurement is unique in the sence that the result covers the wide range of the neutrino energy from $0.25 \mathrm{GeV}$ to $\sim 3 \mathrm{GeV}$. These cross section results are essential inputs for the current and future neutrino oscillation experiments which uses neutrino beam around $1 \mathrm{GeV}$, such as MiniBooNE, T2K and $\mathrm{NO} \nu \mathrm{A}$.

This CC inclusive cross section is nearly free from the uncertainty of the FSI, and hence is complementary to other exclusive measurements. Especially, this result is essential to interpret the MiniBooNE' CC-QE measurements in the $1 \mathrm{GeV}$ region, which reported a significantly higher cross section than what expected from a higher energy measurement by NOMAD.

The total CC interaction cross section values with reliable systematic uncertainties, as defined in our previous cross section ratio measurements for charged current coherent pion production 68. and neutral current neutral pion production [69, 70], are also extracted. These are especially important for the neutrino oscillation experiments in the $1 \mathrm{GeV}$ region, since the $\mathrm{CC}$ pion production and $\mathrm{NC}$ pion production are the the largest backgrounds for $\nu_{\mu}$ disappearance and $\nu_{e}$ appearance measurements, respectively. We extracted a CC interaction cross sections with the same definition used in the previous analyses, and computed the absolute cross sections of these interaction modes. Then, precise comparison with various cross section models become possible, and again, it will help MiniBooNE, T2K and $\mathrm{NO} \nu \mathrm{A}$.

Finally, we also use this result to constrain the neutrino interaction rate for a SciBooNEMiniBooNE joint $\nu_{\mu}$ disappearance analysis. The detail of this oscillation analysis will be described in the next chapter. 


\section{Chapter 7}

\section{Search for muon neutrino disappearance}

\subsection{Overview}

In this chapter, we report a search for muon neutrino disappearance at the Fermilab Booster Neutrino Beam, improved by using SciBooNE data to constrain the uncertainties of neutrino flux and cross sections.

In this analysis, we tune and constrain the MC prediction of the MiniBooNE observables using the result of the SciBooNE CC interaction measurement (Sec. 6.2). The NUANCE based result of SciBooNE measurement is used for this analysis to match the simulation used in MiniBooNE.

First, we describe the MiniBooNE event selection and systematic uncertainties before applying the constraints by SciBooNE in Sec. 7.2. The MiniBooNE data sample used for this analysis is summarized in Sec.7.3. Then, we describe the method how to constrain the systematic uncertainties by using SciBooNE measurements and the final error estimation in Sec. 7.4. The prediction of the oscillation signals and the fit method are described in Sec. 7.5. Finally, the oscillation sensitivity and the result is given in Sec. 7.6, and Sec. 7.7, respectively. The summary of this muon neutrino disappearance measurement is given in Sec. 7.8

\subsection{MiniBooNE event reconstruction and prediction}

We select events in MiniBooNE by requiring a single muon with the decay electron. Neutrino energy is reconstructed from muon kinematics assuming the CCQE interaction by using Eq. (5.2). For the neutrino interaction simulation in the MiniBooNE detector, we use the NUANCE program library with the same parameter set described in Sec. 4.2.2. The details of MiniBooNE event reconstruction and prediction are described in Appendix $\mathrm{C}$.

Figure 7.1 shows the prediction and its systematic uncertainties of the reconstructed $E_{\nu}$ distribution with null oscillation hypothesis, before applying the constraint by SciBooNE measurements The systematic uncertainties are categorized into (a) the flux and cross section uncertainties and (b) the MiniBooNE detector response uncertainty. We see that the dominant uncertainty is from the flux and cross section uncertainties, which can be reduced by using SciBooNE measurement. In the following part of this chapter, we describe the method of reducing of the flux and cross section uncertainties, and an improved search for $\nu_{\mu}$ disappearance.

\subsection{MiniBooNE data sample}

As described in Sec. 3.2, there are two data sets of MiniBooNE neutrino mode running, which we refer to as the "old" and "new" data sets: 


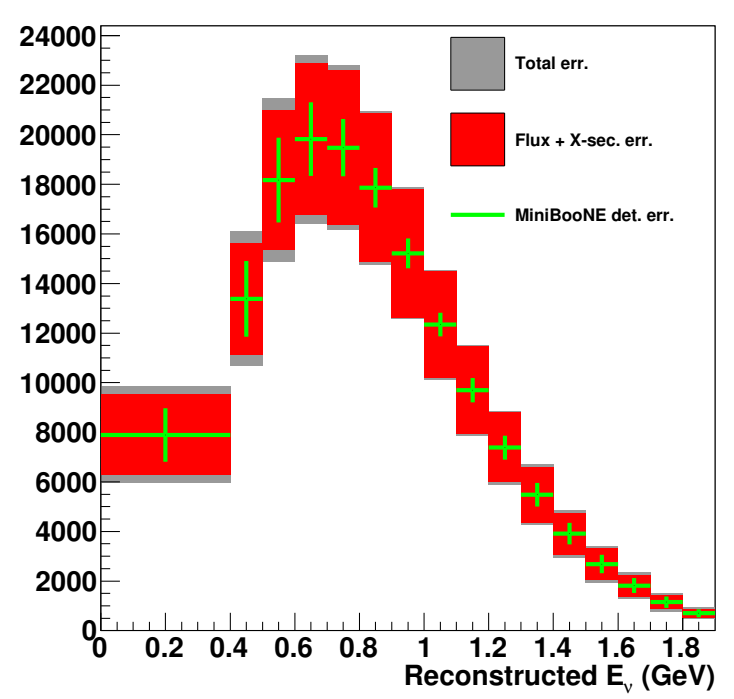

Figure 7.1: Prediction of the MiniBooNE reconstructed $E_{\nu}$ distribution before applying the constraints by SciBooNE measurement. The total error, the flux and cross section uncertainties, and the MiniBooNE detector uncertainty are also shown.

Old data: From Sep. 2002 to Dec. 2005. MiniBooNE only run. $5.58 \times 10^{20}$ POT.

New data: From Oct. 2007 to Apr. 2008. Joint SciBooNE and MiniBooNE run. $0.83 \times 10^{20}$ POT.

The old data is the exactly same sample used for the MiniBooNE only $\nu_{\mu}$ disappearance analysis [44. The new data was taken in the SciBooNE and MiniBooNE joint run period. Although the statistics of the new data is much smaller than that of the old data, this sample has smaller systematic uncertainty for this analysis because both detectors measured the neutrino beam at the same time. This is because errors due to instability of the beamline instruments cancel by taking ratio between SciBooNE and MiniBooNE.

Since the systematic uncertainties are different, we treat these two samples as separate samples in the oscillation analysis.

\subsection{Prediction of MiniBooNE events with the SciBooNE con- straints}

\subsubsection{Central value prediction}

The rate normalization factor obtained by the SciBooNE CC analysis $\left(f_{i}\right)$ are used to constrain the prediction of the reconstructed neutrino energy $\left(E_{\nu}^{R e c}\right)$ distribution at MiniBooNE. First, we generate the MC prediction of $E_{\nu}^{R e c}$ distribution in each true neutrino energy region; $m_{i j}$, where $i$ denotes the index of true energy regions used for the SciBooNE CC analysis, and $j$ denotes the the index of MiniBooNE $E_{\nu}^{R e c}$ bins. Then, the prediction of the number of events in $j$-th $E_{\nu}^{R e c}$ bin, $M_{j}$, is calculated as

$$
M_{j}^{\text {pred }}=\sum_{i} f_{i} m_{i j}
$$

Here, we use $m$ and $M$ to show the number of events in MiniBooNE distributions, to distinguish from the distributions of SciBooNE which are shown with $n$ or $N$. 
We use the same five true energy regions in SciBooNE analysis (Table 6.1), and $16 E_{\nu}^{R e c}$ regions defined in Table 7.1. Figure 7.2 shows the distribution of MiniBooNE $E_{\nu}^{R e c}$ for each true $E_{\nu}$ region, $m_{i j}$. Since the prediction of $m_{i j}$ is produced by the NUANCE based MC simulation, we use $f_{i}$ obtained by NUANCE consistently. The $f_{i}$ are shown in Table 6.4.

Table 7.1: MiniBooNE reconstructed $E_{\nu}$ bins

\begin{tabular}{cccccc}
\hline \hline bin & 1 & 2 & 3 & $\cdots$ & 16 \\
\hline$E_{\nu}^{r e c}(\mathrm{GeV})$ & $0.0-0.4$ & $0.5-0.6$ & $0.6-0.7$ & $(0.1 \mathrm{GeV}$ steps $)$ & $1.8-1.9$ \\
\hline \hline
\end{tabular}

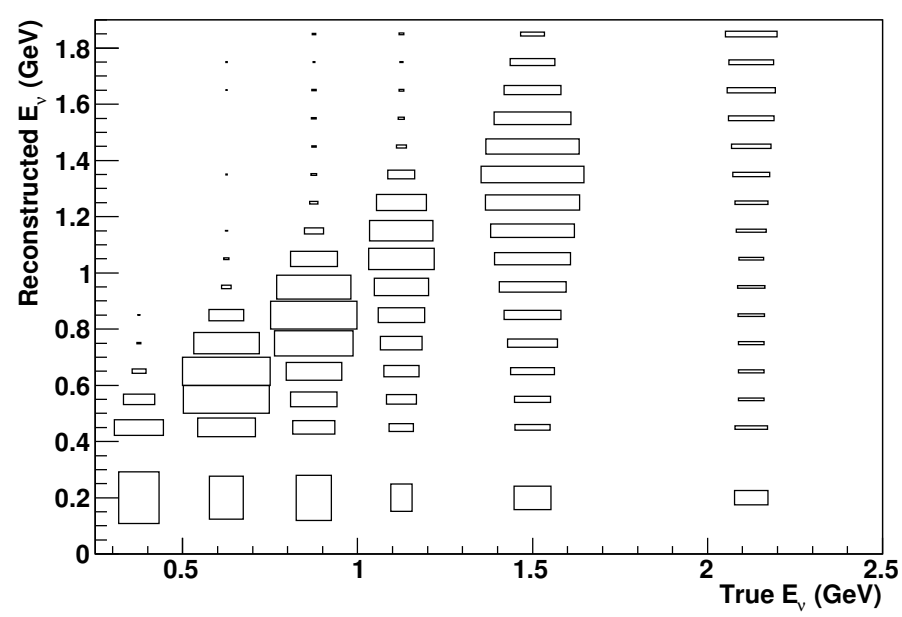

Figure 7.2: Distribution of the MiniBooNE reconstructed $E_{\nu}$ versus true $E_{\nu}$.

\subsubsection{Systematic uncertainties}

We estimate the systematic uncertainty of the MiniBooNE reconstructed $E_{\nu}$ distribution for each reconstructed $E_{\nu}$ bin, including bin-to-bin correlations, and form a error matrix $\mathbf{V}^{\text {sys }}$.

The sources of systematic errors are categorized into the following components:

1. Flux and cross section uncertainties

(a) Errors of the SciBooNE CC interaction measurement $\left(\mathbf{V}^{S B}\right)$

(b) Errors of the ratio between SciBooNE and MiniBooNE distributions ( $\mathbf{V}^{\text {far } / \text { near }}$ )

2. MiniBooNE detector response uncertainties $\left(\mathbf{V}^{\text {det }}\right)$

3. Errors due to long term instabilities $\left(\mathbf{V}^{s t a b}\right)$

As for the flux and cross section uncertainties, they are mostly cancelled by taking the ratio between SciBooNE and MiniBooNE distributions. However, as listed above, there are remaining errors due to (a) errors of the SciBooNE CC interaction measurement, and (b) errors of the SciBooNE to MiniBooNE ratio due to flux differences and efficiency differences by cross section uncertainties.

Then, the total systematic error, $\mathbf{V}^{\text {sys }}$, is constructed by adding these together:

$$
\mathbf{V}^{\text {sys }}=\mathbf{V}^{S B}+\mathbf{V}^{\text {far } / \text { near }}+\mathbf{V}^{\text {det }}+\mathbf{V}^{\text {stab }} .
$$


The systematic errors for oscillated predictions are obtained by re-scaling the errors for null oscillation prediction. Then, for easier calculation of the re-scaled error matrix, we define the "fractional error", $V^{\text {frac }}$, as

$$
V_{j k}^{f r a c}=\frac{V_{j k}^{a b s}}{M_{j} M_{k}},
$$

where $j, k$ are the indexes of reconstructed $E_{\nu}$ bins, $M_{j}, M_{k}$ are the predicted number of events, $V_{j k}^{a b s}$ is a element of the absolute error matrix. The error matrices appeared in this chapter always represents the size of fractional uncertainties unless otherwise noted.

Among these errors, MiniBooNE detector error $\left(\mathbf{V}^{\text {det }}\right)$ is same as the error used for the MiniBooNE only analysis, and is described in Appendix C.1.1 and elsewhere [82, 108. In the following part, we describe the method of estimating other three errors $\left(\mathbf{V}^{S B}, \mathbf{V}^{\text {far/near }}\right.$ and $\left.\mathbf{V}^{\text {stab }}\right)$ in detail.

\subsubsection{Errors of the SciBooNE CC interaction measurement}

We estimate the errors of the SciBooNE CC interaction measurement by propagating the errors of the rate normalization factor, $f_{i}$, to the MiniBooNE distribution, $M_{j}$. The errors of $f_{i}$ are given in Table 6.4.

We first produce a random set of the rate normalization factors, $f_{i}^{\prime}$, drown from their error. Then, systematically varied prediction of MiniBooNE, $M_{j}^{\prime}$, corresponding to the set of $f_{i}^{\prime}$, are obtained as:

$$
M_{j}^{\prime}=\sum_{j} f_{i}^{\prime} m_{i j}
$$

We produce 1000 sets of $M_{i}^{\prime}$, and the error matrix by the SciBooNE CC measurement is calculated as:

$$
V_{j k}^{S B}=\frac{1}{S} \sum_{l}^{S} \frac{\left(M_{j l}^{\prime}-M_{j}\right)}{M_{j}} \frac{\left(M_{k l}^{\prime}-M_{k}\right)}{M_{k}},
$$

where $l$ denotes the index of random draws and $S$ denotes the total number of draws.

\subsubsection{Errors of the ratio between SciBooNE and MiniBooNE distributions}

The systematic error of SciBooNE to MiniBooNE ratio resulting from the flux and cross section uncertainties is estimated in a way similar to estimating the errors of the SciBooNE CC interaction measurement (Sec. 6.2.3). The sources of errors taken into account are (i) and (ii) in Table 6.2.

The errors are constrained by the SciBooNE CC measurement. The constrained systematic variations of the flux and cross section uncertainties for the MiniBooNE distribution are estimated in the following way.

First, we generate a new prediction corresponding to a systematic variation of the MiniBooNE distribution, $m_{i j}^{\prime}$. Here, the prime denotes values with systematic variation. Then, the predicted number of events, $M_{j}^{\prime}$, corresponding to this systematic variation is:

$$
M_{j}^{\prime}=\sum_{i} f_{i} m_{i j}^{\prime} R_{i}^{S B}
$$

where $R_{i}^{S B}$ is the renormalization factor, which is the same values used in the SciBooNE analysis:

$$
R_{i}^{S B}=\frac{\sum_{j}^{\left(p_{\mu}, \theta_{\mu}\right) b i n s} n_{i j}}{\sum_{j}^{\left(p_{\mu}, \theta_{\mu}\right) b i n s} n_{i j}^{\prime}} .
$$

This renormalization factor removes the error for absolute normalization for each true neutrino energy region. To see the meaning of Eq. (7.6) further, we rewrite this equation as:

$$
M_{j}^{\prime}=\sum_{i}\left(f_{i} m_{i j}^{\prime} R_{i}^{M B} \times \frac{R_{i}^{S B}}{R_{i}^{M B}}\right),
$$


where $R_{i}^{M B}$ is the renormalization factor for the number of MiniBooNE events, defined as:

$$
R_{i}^{M B}=\frac{\sum_{j} m_{i j}}{\sum_{j} m_{i j}^{\prime}}
$$

Here, one can see that the first term of Eq. (7.8), $f_{i} m_{i j}^{\prime} R_{i}^{M B}$, represents the "shape" variation analogous to Eq. 6.4), and the second term, $R_{i}^{S B} / R_{i}^{M B}$, represents the variation of the SciBooNE to MiniBooNE ratio due to the flux and cross section uncertainties.

If we take an example of the error from $M_{A}^{Q E}$ uncertainty, we set 1000 different values of $M_{A}^{Q E}$ randomly drawn from the estimated uncertainty of $M_{A}^{Q E}$, and generate the corresponding MC expectations of $n_{i j}^{\prime}$ and $m_{i j}^{\prime}$. Then, the error matrix that represents the uncertainty of $M_{A}^{Q E}$ is calculated as:

$$
V_{j k}^{M_{A}^{Q E}}=\frac{1}{S} \sum_{l}^{S} \frac{\left(M_{j l}^{\prime}-M_{j}\right)}{M_{j}} \frac{\left(M_{k l}^{\prime}-M_{k}\right)}{M_{k}},
$$

where $l$ denotes the index of random draws and $S$ denotes the total number of draws.

The total SciBooNE to MiniBooNE ratio error is constructed by adding each source together:

$$
\mathbf{V}^{\text {far } / \text { near }}=\mathbf{V}^{M_{A}^{Q E}}+\mathbf{V}^{\pi^{+} \text {production }}+\cdots .
$$

\subsubsection{Stability errors}

We also include the error due to instability of the MiniBooNE data over the time. This error is especially important since SciBooNE data only covers a partial period of the MiniBooNE data taking.

The following sources are taken into account.

- MiniBooNE reconstructed energy scale

- Horn current

- POT measurement

Among these, the horn current error $\left(\mathbf{V}^{\text {horn }}\right)$ and the POT error $\left(\mathbf{V}^{P O T}\right)$ for the new data cancel by taking the ratio with the SciBooNE data, and are included only in the old data error. The energy scale error $\left(\mathbf{V}^{\text {Escale }}\right)$ is assigned for both new and old data sets.

Hence the stability errors for the new and old data sets $\left(\mathbf{V}^{\text {stab(new) }}\right.$ and $\left.\mathbf{V}^{\text {stab(old) }}\right)$ are calculated as:

$$
\mathbf{V}^{s t a b(n e w)}=\mathbf{V}^{\text {Escale }},
$$

and

$$
\mathbf{V}^{\text {stab(old })}=\mathbf{V}^{\text {Escale }}+\mathbf{V}^{\text {horn }}+\mathbf{V}^{P O T} .
$$

The followings are more details about these stability errors.

Energy scale instability The absolute energy scale of the MiniBooNE detector is continuously monitored by using the decay electrons from the stopped muons. Figure 7.3 shows the long term stability of the MiniBooNE reconstructed energy scale since the beginning of the MiniBooNE data taking. We find that, although it is pretty stable, there is about $1 \%$ level drift over the entire beam data taking period. Hence we set $1 \%$ uncertainty for the energy scale both for new and old data.

Horn current instability Figure 7.4 shows the current on the horn in the neutrino mode data taking. We see that there is about $\pm 1 \mathrm{kA}$ fluctuation of the horn current. Hence, we set $\pm 1 \mathrm{kA}$ horn current uncertainty for the old data prediction. 

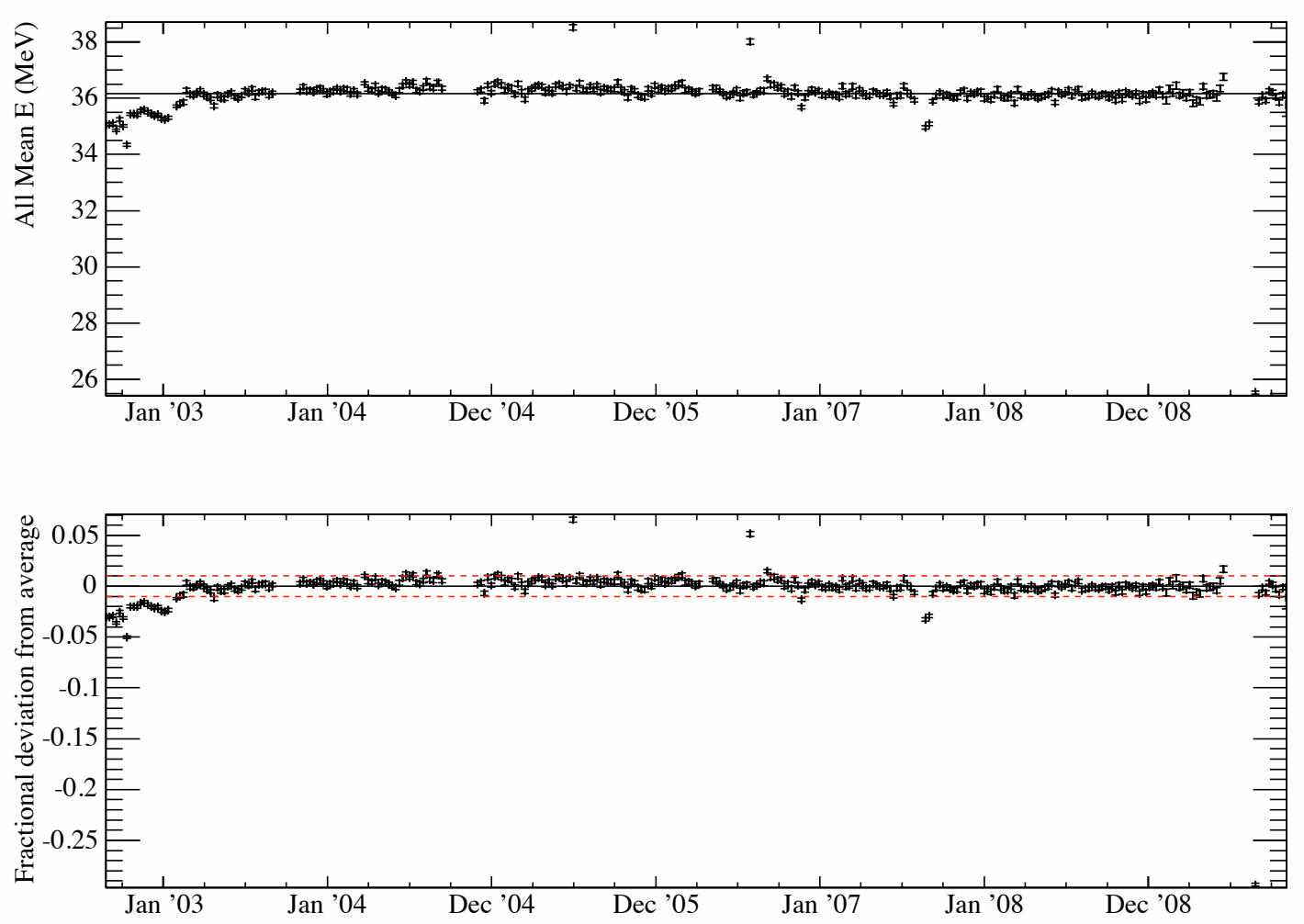

Figure 7.3: Stability of the MiniBooNE reconstructed energy scale of the decay electrons from the stopped muons since the beginning of the beam data taking in 2002. The top plot show the absolute scale, and the bottom plot show the deviation from the average. The red dashed lines indicate $\pm 1 \%$ deviation. This plot is by C. Green and H. Ray. 

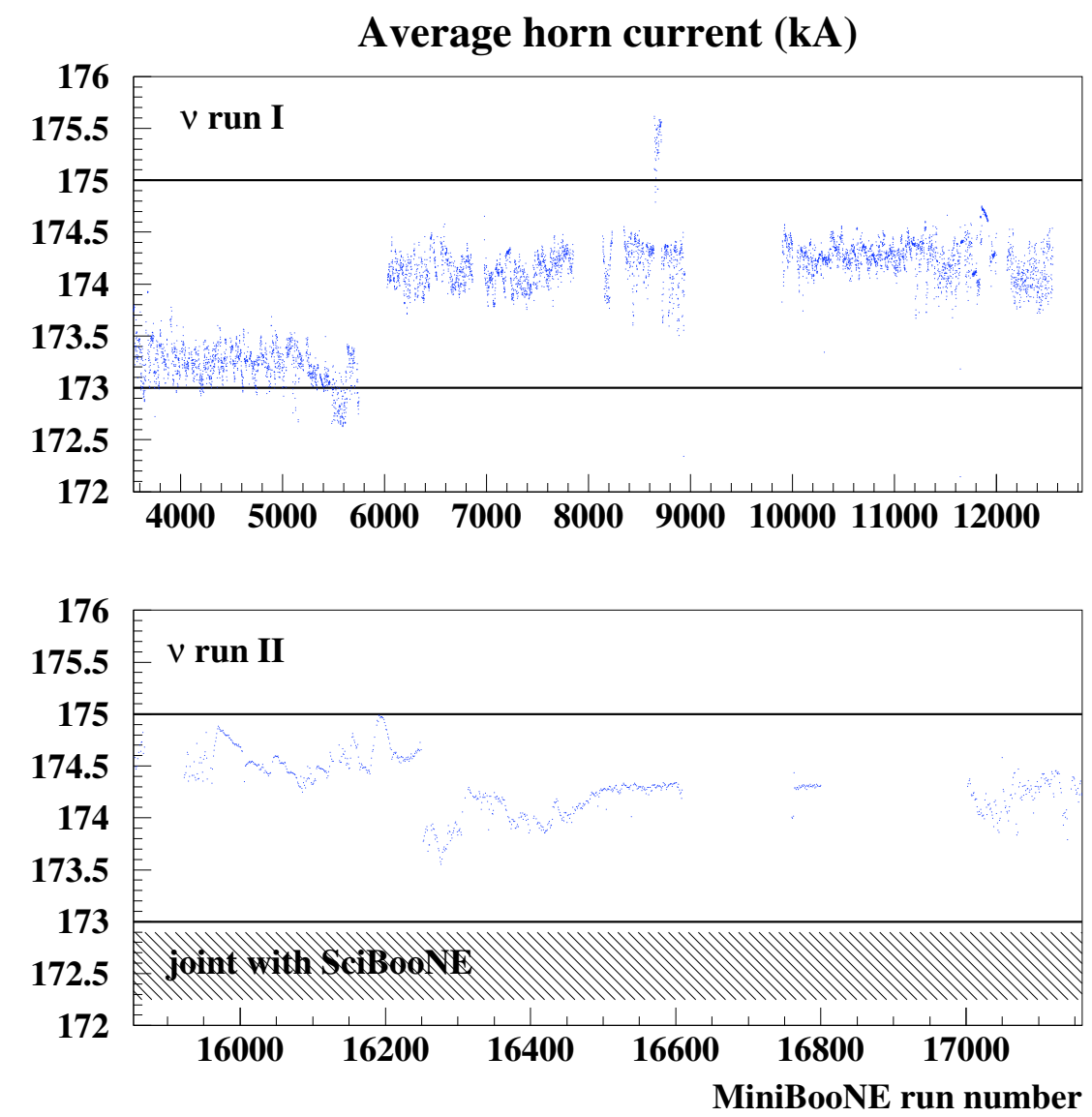

Figure 7.4: Stability of the horn current (kA) for the MiniBooNE neutrino mode running. The top plot shows the current during the first neutrino-mode run with MiniBooNE only, and the bottom plot shows that of the second neutrino-mode run with both SciBooNE and MiniBooNE. Plots are taken from Ref. [82]. 
POT measurement error The number of POT is calculated by the beam current measured by using the toroidal coils placed in the proton beam line. The uncertainty of the stability of the toroid measurements is estimated to be $2 \%$. Hence we set $2 \%$ pure normalization uncertainty for the old data set.

\subsubsection{Final prediction}

Figure 7.5 shows the predictions of MiniBooNE reconstructed $E_{\nu}$ for the old data after applying the SciBooNE constraint. Figure 7.6 shows the size of the diagonal elements of the error matrices $\left(\sqrt{V_{i i}}\right)$ for each reconstructed $E_{\nu}$ bin. The flux and cross section errors become significantly smaller by applying constraint of SciBooNE, to the same level with the MiniBooNE detector uncertainty. The correlation coefficients of the total systematic uncertainties for each reconstructed $E_{\nu}$ bin, $V_{i j}^{s y s} / \sqrt{V_{i i}^{s y s} V_{j j}^{s y s}}$, are shown in Fig. 7.7.

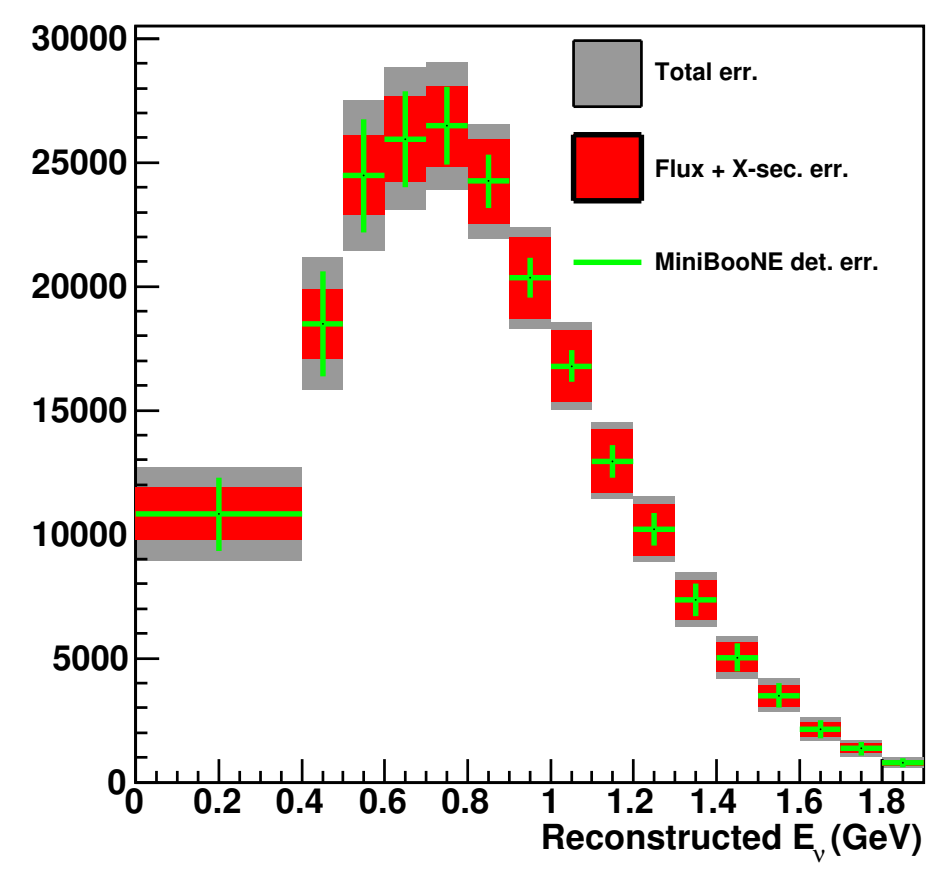

Figure 7.5: The distribution of the MiniBooNE reconstructed $E_{\nu}$ after applying the SciBooNE constraint. The total error, and contributions of the flux and cross section uncertainties and of the MiniBooNE detector uncertainty are also shown.

Figure 7.8 shows the size of errors by the flux and cross section uncertainties. For this analysis, the flux error becomes negligible by taking the ratio between SciBooNE and MiniBooNE. On the other hand, the cross section uncertainty is one of the dominant sources of the systematic error, together with the MiniBooNE detector response uncertainties. The major reason of this large cross section uncertainties is that SciBooNE and MiniBooNE have different acceptances as a function of the muon angle; SciBooNE has more acceptance to forward-going muons while MiniBooNE has isotropic acceptance. Since the variation of the cross section model affect the muon angler distributions, it changes the efficiencies at SciBooNE and MiniBooNE differently. 


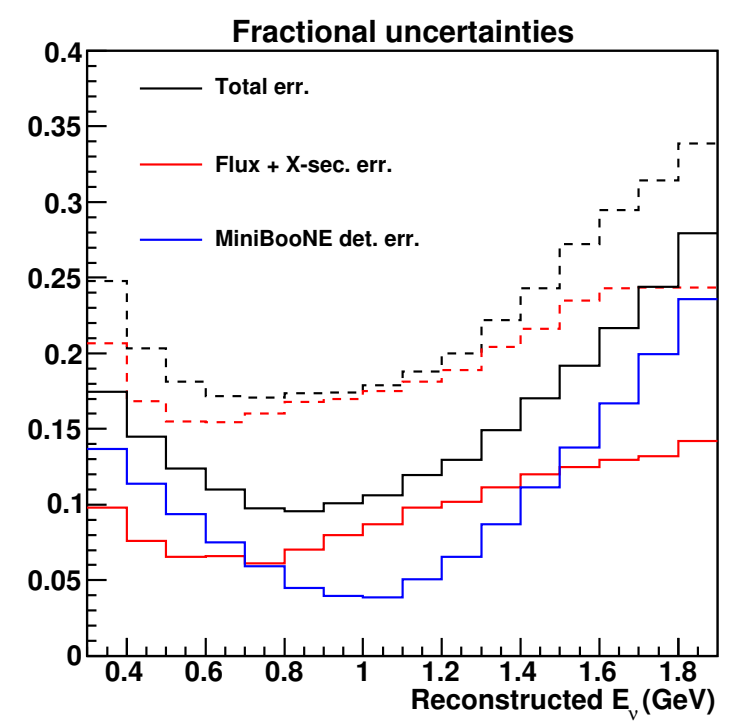

Figure 7.6: The size of diagonal elements $\left(\sqrt{V_{i i}}\right)$ of the error matrices. The dotted line shows the error before applying the SciBooNE constraint (errors for MiniBooNE only fit). The solid lines show the errors constrained by the SciBooNE measurement, used for this joint analysis.

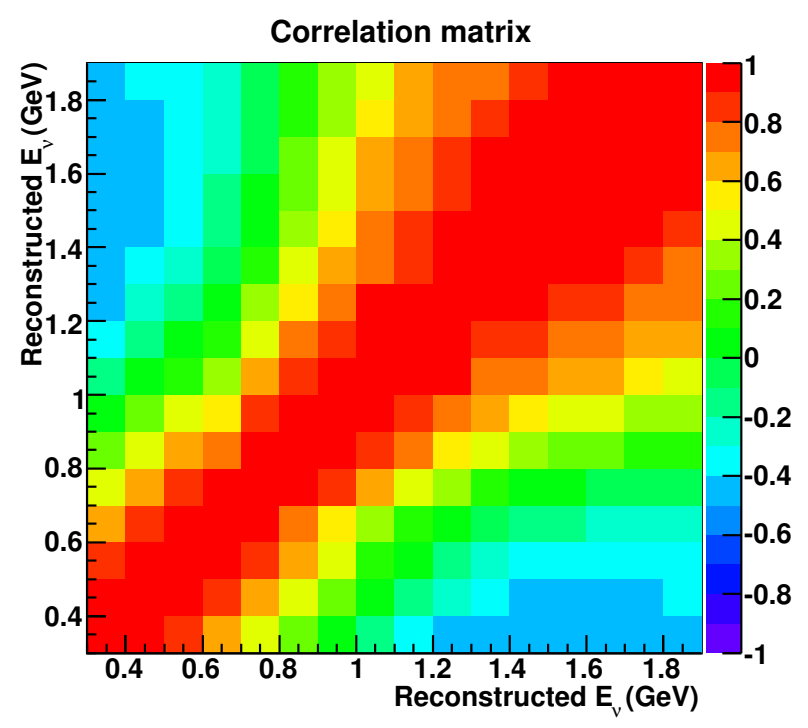

Figure 7.7: The size of the correlation coefficient $\left(V_{i j} / \sqrt{V_{i i} V_{j j}}\right)$ for the total systematic uncertainties on the MiniBooNE reconstructed $E_{\nu}$ distribution. 

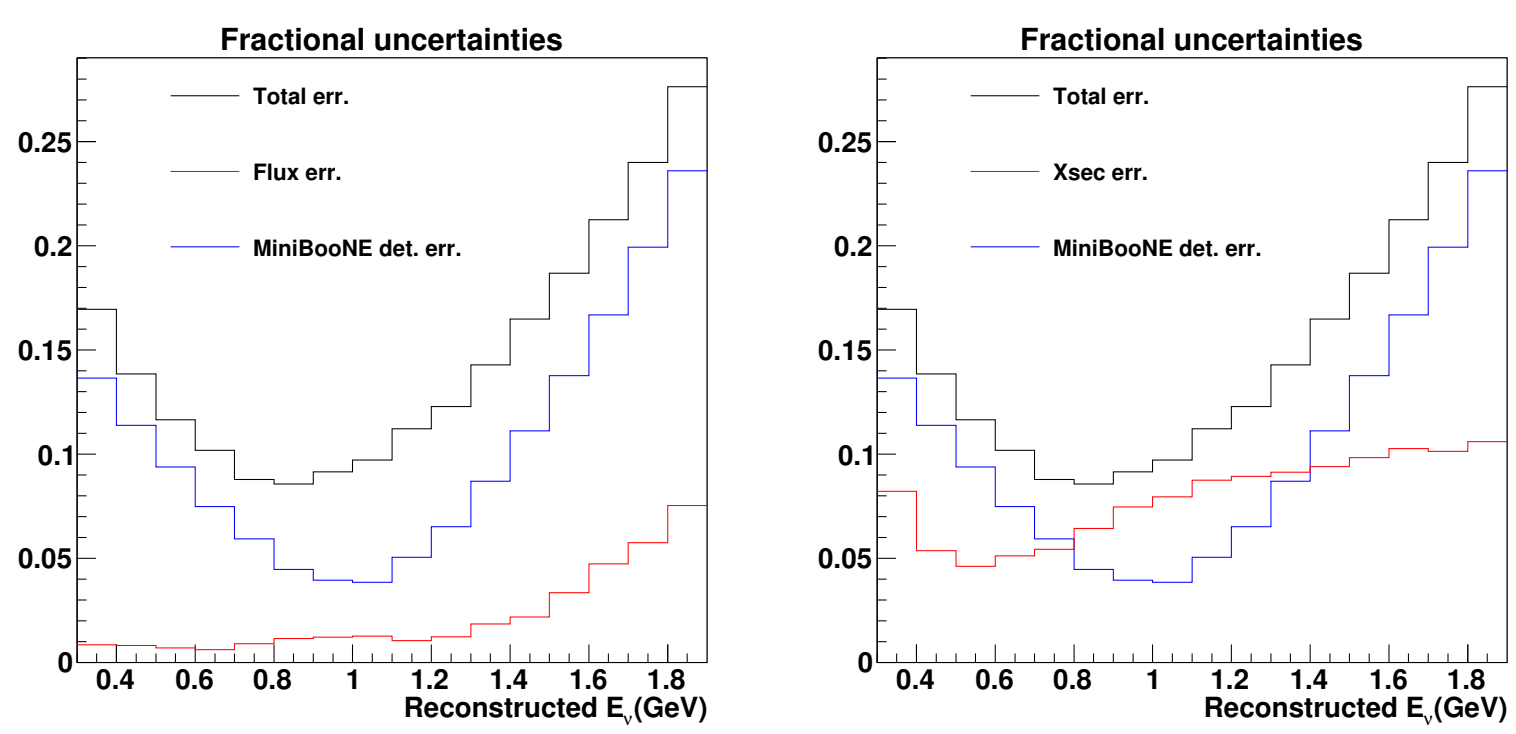

Figure 7.8: The size of diagonal elements $\left(\sqrt{V_{i i}}\right)$ of the error matrices for the MiniBooNE reconstructed $E_{\nu}$ distribution by the flux (left) and the cross section (right) uncertainties. The total and MiniBooNE detector response errors are also shown as a reference.

\subsection{Test of neutrino oscillation hypothesis}

\subsubsection{Prediction of oscillation signal}

\subsubsection{Overview}

We search for muon neutrino disappearance assuming the mixing between 2 neutrino flavors; $\nu_{\mu}$ and $\nu_{x}$. The $\nu_{\mu} \rightarrow \nu_{x}$ disappearance probability is given as

$$
P\left(\nu_{\mu} \rightarrow \nu_{x}\right)=\sin ^{2} 2 \theta \sin ^{2}\left(1.27 \Delta m^{2} \frac{L}{E}\right)
$$

where $\theta$ is the mixing angle, $\Delta m^{2}\left[\mathrm{eV}^{2}\right]$ is the mass splitting between 2 flavors, $\mathrm{L}[\mathrm{km}]$ is the distance traveled and $E[\mathrm{GeV}]$ is the neutrino energy. If we assume an oscillation with the $(3+1)$ sterile neutrino scheme shown in Eq. $(1.26), \sin ^{2} 2 \theta=4\left|U_{\mu 4}\right|^{2}\left(1-\left|U_{\mu 4}\right|^{2}\right)$ and $\Delta m^{2}=\Delta m_{41}^{2}$.

Figure 7.9 shows the expected travel distances of muon neutrinos for the selected events in SciBooNE and MiniBooNE. The travel distance is defined as the 3D distance between the neutrino production point and the neutrino interaction point. The distributions of the travel distances have a width of $50 \mathrm{~m}$, which corresponds to the length of the decay volume. The averaged travel distances are $\sim 76 \mathrm{~m}$ and $\sim 520 \mathrm{~m}$ for SciBooNE and MiniBooNE, respectively. Since the $50 \mathrm{~m}$ width is not negligible compared to the travel distances ( $\sim 76 \mathrm{~m}$ for SciBooNE, $\sim 520 \mathrm{~m}$ for MiniBooNE), we take this effect into account by calculating the oscillation probabilities event-by-event.

Figure 7.10 show the survival probabilities at SciBooNE and MiniBooNE and the ratio for the total number of the selected events. There is a sizable oscillation effect at SciBooNE as well as at MiniBooNE, and hence we should consider oscillations at both of the two detectors. The oscillation probability becomes maximum at the first oscillation peak when $1.27 \Delta m^{2} L /\langle E\rangle \sim \pi / 2$, where $\langle E\rangle$ is the mean neutrino energy, and then it approaches to $1 / 2$ at high $\Delta m^{2}$ region since the effect of oscillation is washed out by taking the energy average.

As described in the previous section, we re-weight the MiniBooNE prediction by the rate normalization factor obtained by the SciBooNE CC measurement. Hence, the expected effect of oscillation is the ratio of the survival probability at MiniBooNE ( $\mathrm{P}(\mathrm{MB})$ ) divided by the survival probability at SciBooNE $(\mathrm{P}(\mathrm{SB}))$, as shown in the bottom plot of Figure 7.10 . Therefore, this 

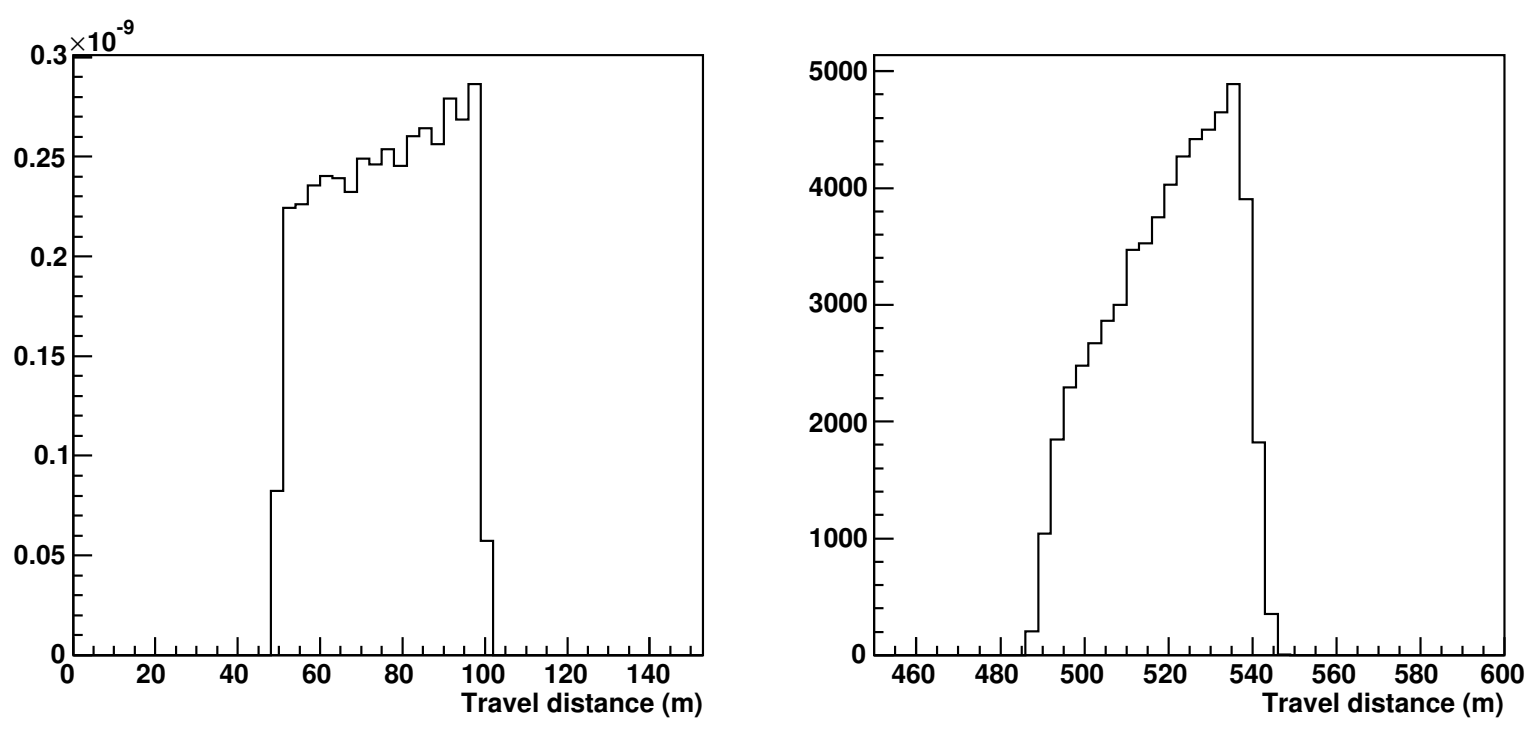

Figure 7.9: Expected travel distances of neutrinos for the selected events at SciBooNE (left) and MiniBooNE (right).

analysis is sensitive to the oscillation where the $\mathrm{P}(\mathrm{MB}) / \mathrm{P}(\mathrm{SB})$ is expected to be apart from 1 , which is $0.5<\Delta m^{2}<30 \mathrm{eV}^{2}$.

\subsubsection{Oscillation effect at SciBooNE}

Since the rate normalization factor $f_{i}$ is obtained by the SciBooNE measurement without assuming the neutrino oscillation, the values of the $f_{i}$ should be varied depending on the oscillation parameters $\left(\sin ^{2} 2 \theta\right.$ and $\left.\Delta m^{2}\right)$. Ideally, such normalization factors with the oscillation effect, $f_{i}\left(\sin ^{2} 2 \theta, \Delta m^{2}\right)$, are to be obtained by performing the spectrum fit described in Sec. 6.2.1 with appropriate MC predictions with oscillation effects. However, it is technically difficult to compute $f_{i}\left(\sin ^{2} 2 \theta, \Delta m^{2}\right)$ for each oscillation parameters to be tested because of our limited computing resources. Hence, we define approximate normalization factors as a function of the oscillation parameters, $\mathcal{F}_{i}\left(\sin ^{2} 2 \theta, \Delta m^{2}\right)$, which satisfies

$$
f_{i}(0,0) \cdot \mathcal{N}_{i}^{\text {pred }}(0,0)=\mathcal{F}_{i}\left(\sin ^{2} 2 \theta, \Delta m^{2}\right) \cdot \mathcal{N}_{i}^{\text {pred }}\left(\sin ^{2} 2 \theta, \Delta m^{2}\right)=(\text { Number of observed events }),
$$

where $i$ is the index of the true neutrino energy regions and $\mathcal{N}_{i}^{\text {pred }}\left(\sin ^{2} 2 \theta, \Delta m^{2}\right)$ is the expected numbers of events in SciBooNE as a function of $\Delta m^{2}$ and $\sin ^{2} 2 \theta$. Here, we note that $f_{i}(0,0)$ and $\mathcal{N}_{i}^{\text {pred }}(0,0)$ are same as $f_{i}$ and $\mathcal{N}_{i}^{\text {pred }}$ defined in Sec. 6 .

To test Eq. (7.15), we perform the spectrum fits with MC predictions with several oscillation parameters $\left(\sin ^{2} 2 \theta, \Delta m^{2}\right)$, by substituting $n_{i j}^{\text {pred }}$ in Eq. 6.1 with oscillated prediction $n_{i j}^{\text {pred }}\left(\sin ^{2} 2 \theta, \Delta m^{2}\right)$. If the spectrum fit works perfectly, we should obtain $f_{i}\left(\sin ^{2} 2 \theta, \Delta m^{2}\right)=$ $\mathcal{F}_{i}\left(\sin ^{2} 2 \theta, \Delta m^{2}\right)$.

To see the consistency of $f_{i}\left(\sin ^{2} 2 \theta, \Delta m^{2}\right)$ and $\mathcal{F}_{i}\left(\sin ^{2} 2 \theta, \Delta m^{2}\right)$, we compare

$$
\frac{\mathcal{N}_{i}^{\text {pred }}\left(\sin ^{2} 2 \theta, \Delta m^{2}\right)}{\mathcal{N}_{i}^{\text {pred }}(0,0)}=\frac{f_{i}(0,0)}{\mathcal{F}_{i}\left(\sin ^{2} 2 \theta, \Delta m^{2}\right)} \text { and } \frac{f_{i}(0,0)}{f_{i}\left(\sin ^{2} 2 \theta, \Delta m^{2}\right)},
$$

which both represent the oscillation probability at each oscillation parameter set, as shown in Figure 7.11. We see a fairly good agreement between $f_{i}\left(\sin ^{2} 2 \theta, \Delta m^{2}\right)$ and $\mathcal{F}_{i}\left(\sin ^{2} 2 \theta, \Delta m^{2}\right)$. Hence, we use $\mathcal{F}_{i}\left(\sin ^{2} 2 \theta, \Delta m^{2}\right)=f_{i}(0,0) \cdot \mathcal{N}_{i}^{\text {pred }}(0,0) / \mathcal{N}_{i}^{\text {pred }}\left(\sin ^{2} 2 \theta, \Delta m^{2}\right)$ to predict the oscillation effect at SciBooNE. 

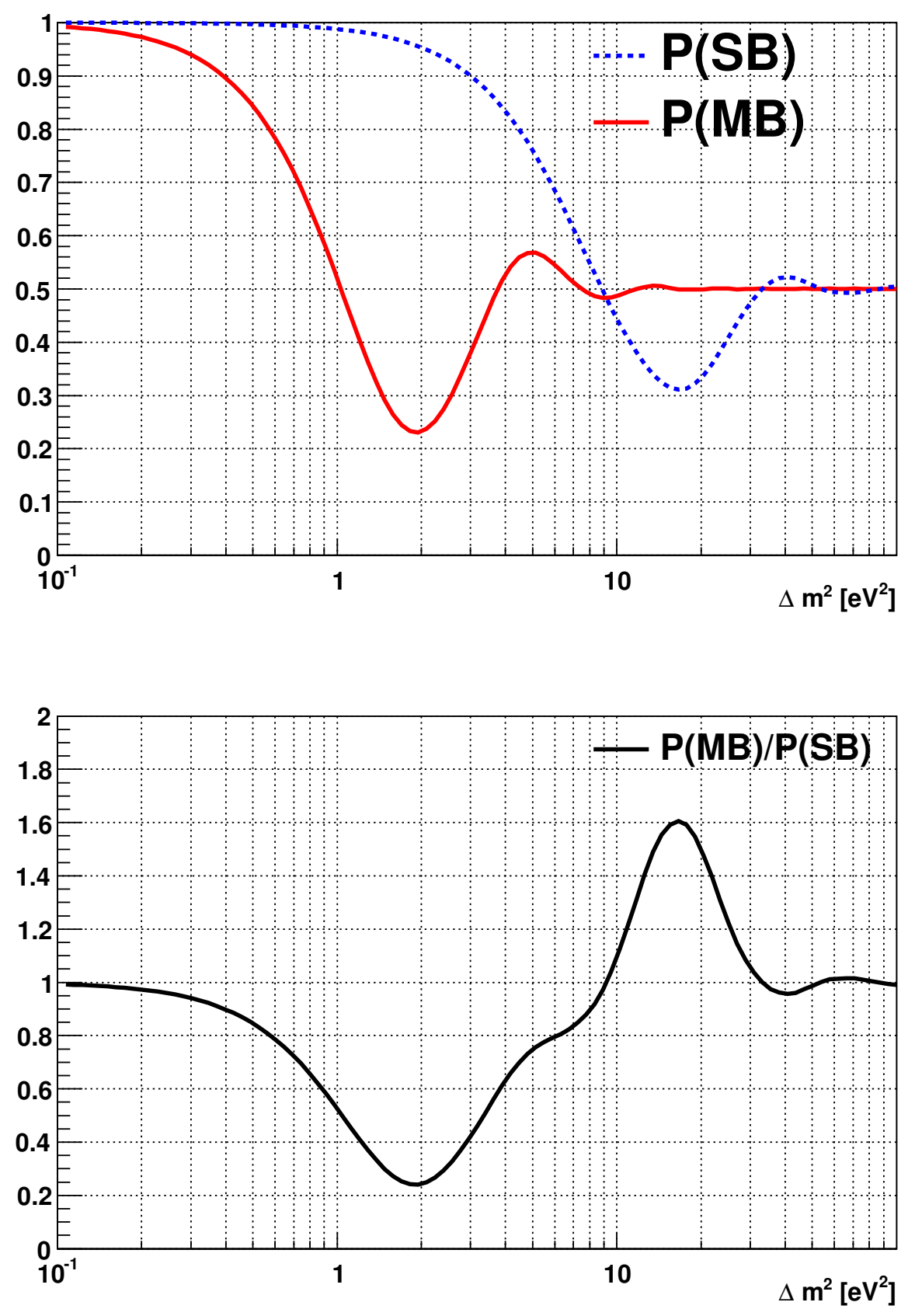

Figure 7.10: (Top) Survival probabilities of the total number of the selected events at SciBooNE $(\mathrm{P}(\mathrm{SB}))$ and MiniBooNE $(\mathrm{P}(\mathrm{MB}))$ as a function of $\Delta m^{2}$. (Bottom) The ratio of the number of events at MiniBooNE and SciBooNE $(\mathrm{P}(\mathrm{MB}) / \mathrm{P}(\mathrm{SB}))$. The value of $\sin ^{2} 2 \theta$ is set to 1 . 


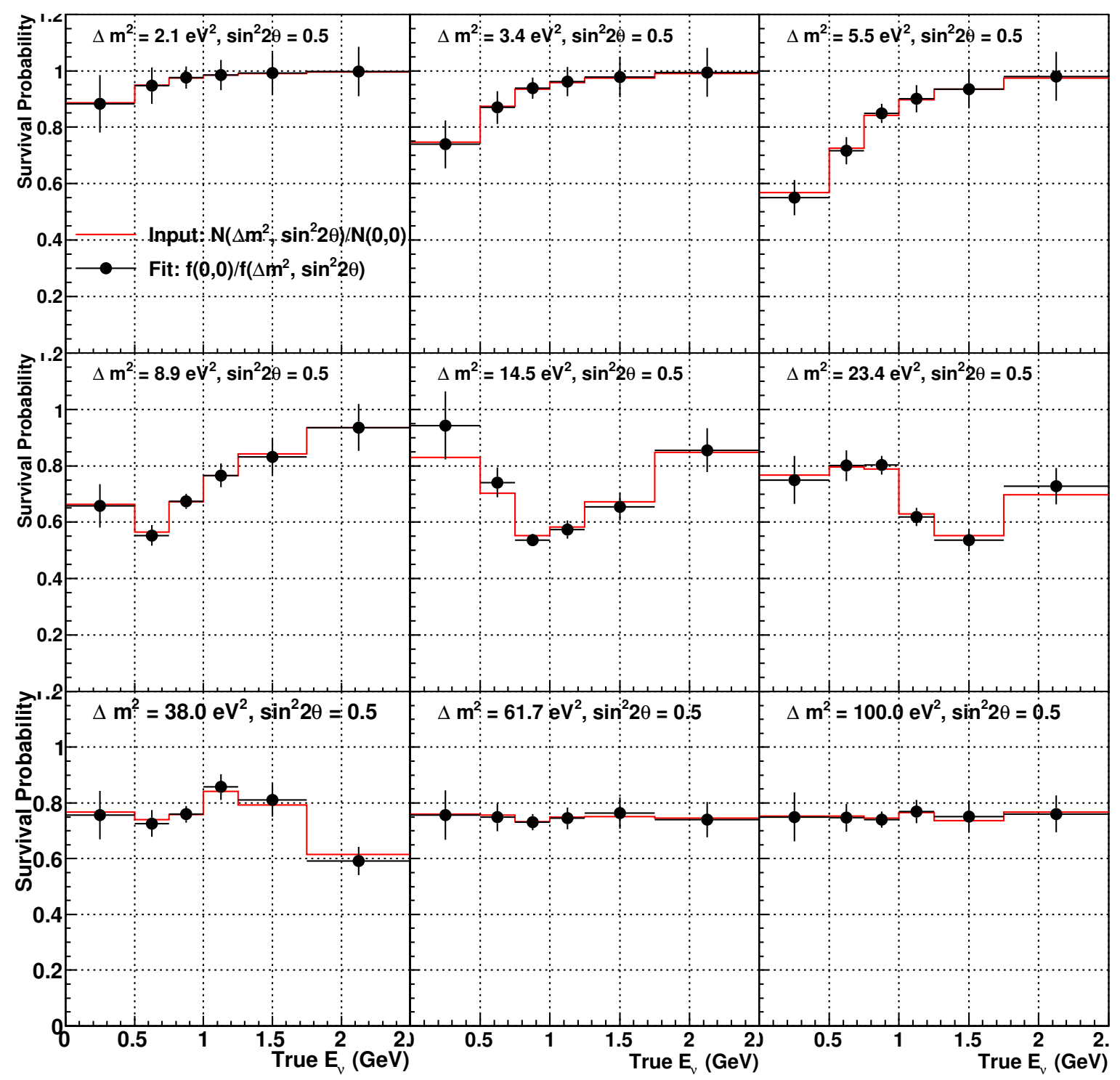

Figure 7.11: Spectrum fit results for MC predictions with various sets of oscillation parameters. The solid red lines show the input values $\left(\mathcal{N}_{i}^{\text {pred }}\left(\sin ^{2} 2 \theta, \Delta m^{2}\right) / \mathcal{N}_{i}^{\text {pred }}(0,0)=\right.$ $\left.f_{i}(0,0) / \mathcal{F}_{i}\left(\sin ^{2} 2 \theta, \Delta m^{2}\right)\right)$ and the points show the fit results $\left(f_{i}(0,0) / f_{i}\left(\sin ^{2} 2 \theta, \Delta m^{2}\right)\right)$. 


\subsubsection{Oscillation prediction at MiniBooNE}

We make the predictions of MiniBooNE by computing $f_{i}$ and $m_{i j}$ in Eq. (7.1) for each set of $\left(\sin ^{2} 2 \theta, \Delta m^{2}\right)$.

The predictions of MiniBooNE for $i$-th true energy bin and $j$-th reconstructed energy bin, $m_{i j}\left(\sin ^{2} 2 \theta, \Delta m^{2}\right)$, is produced by re-weighting the MC prediction with their oscillation probability event-by-event. Then, using $\mathcal{F}_{i}\left(\sin ^{2} 2 \theta, \Delta m^{2}\right)$ described in the previous section, the prediction of MiniBooNE $E_{\nu}^{r e c}$ distribution, $M_{j}^{\text {pred }}\left(\sin ^{2} 2 \theta, \Delta m^{2}\right)$, is obtained as

$$
\begin{aligned}
M_{j}^{\text {pred }}\left(\sin ^{2} 2 \theta, \Delta m^{2}\right) & =\sum_{i}^{E_{\nu} \text { bins }} \mathcal{F}_{i}\left(\sin ^{2} 2 \theta, \Delta m^{2}\right) \cdot m_{i j}\left(\sin ^{2} 2 \theta, \Delta m^{2}\right) \\
& =\sum_{i}^{E_{\nu} \text { bins }} f_{i}(0,0) \frac{\mathcal{N}_{i}^{\text {pred }}(0,0)}{\mathcal{N}_{i}^{\text {pred }}\left(\sin ^{2} 2 \theta, \Delta m^{2}\right)} \cdot m_{i j}\left(\sin ^{2} 2 \theta, \Delta m^{2}\right) .
\end{aligned}
$$

Figure 7.12 show the predictions of MiniBooNE reconstructed $E_{\nu}$ for several sets of oscillation parameters. We search for oscillation signals by testing these predictions with the MiniBooNE measurements.

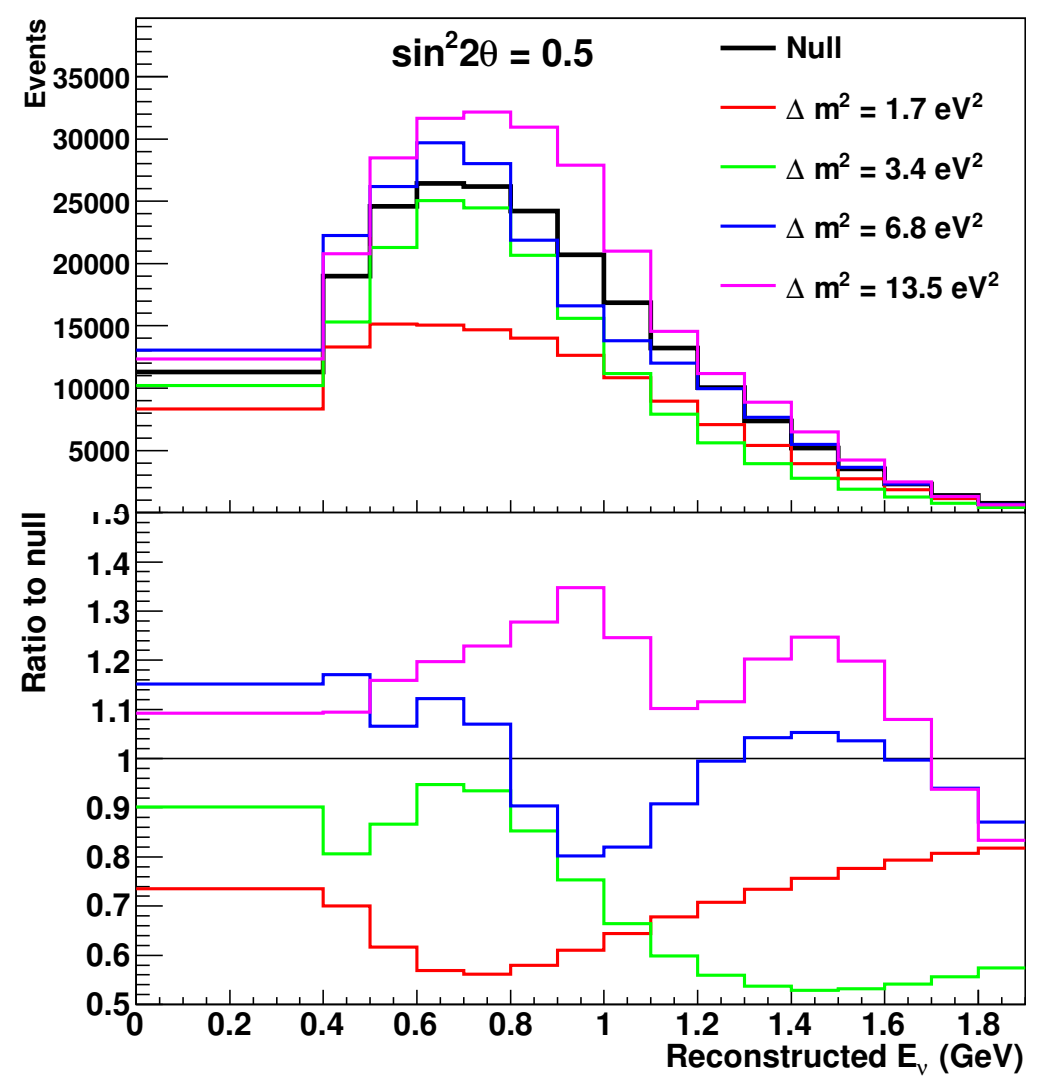

Figure 7.12: Predictions of MiniBooNE reconstructed $E_{\nu}$ distributions with several sets of oscillation parameters. The top plot show the expected number of events and the bottom plot shows the ratio to the null oscillation prediction. Predictions for $\Delta m^{2}=1.7,3.4,6.8$ and $13.5\left(\mathrm{eV}^{2}\right)$ are shown. The value of $\sin ^{2} 2 \theta$ is set to 0.5 for all oscillation predictions. 


\subsubsection{Definition of the $\chi^{2}$}

We fit the MiniBooNE $E_{\nu}^{R e c}$ distribution to find the best fit parameter minimizing the $\chi^{2}$ value. As described in Sec. 7.3, we have two kinds of data sets ("new" and "old" samples) with different systematic uncertainties. Hence, we treat these two as separate samples. For the final result, we fit both "new" and "old" data sets simultaneously. Here, we first describe the definition of the $\chi^{2}$ for a "new" data fit and then describe about the combined fit.

\subsubsection{Fit for the new data}

The $\chi^{2}$ for the new data fit is defined as:

$$
\chi^{2}=\sum_{j, k}^{16 \text { bins }}\left(\frac{M_{j}^{\text {obs }}-M_{j}^{\text {pred }}\left(\sin ^{2} 2 \theta, \Delta m^{2}\right)}{M_{j}^{\text {pred }}\left(\sin ^{2} 2 \theta, \Delta m^{2}\right)}\right) V_{j k}^{-1}\left(\sin ^{2} 2 \theta, \Delta m^{2}\right)\left(\frac{M_{k}^{\text {obs }}-M_{k}^{\text {pred }}\left(\sin ^{2} 2 \theta, \Delta m^{2}\right)}{M_{k}^{\text {pred }}\left(\sin ^{2} 2 \theta, \Delta m^{2}\right)}\right)
$$

where $i, j$ denote $E_{\nu}^{R e c}$ bins, $M_{j(k)}^{\text {obs }}$ and $M_{j(k)}^{\text {pred }}\left(\sin ^{2} 2 \theta, \Delta m^{2}\right)$ respectively denote the observed and the predicted number of events at each bin, and $V_{j k}\left(\sin ^{2} 2 \theta, \Delta m^{2}\right)$ represents the statistical and systematic uncertainties. The error matrix $V_{j k}\left(\sin ^{2} 2 \theta, \Delta m^{2}\right)$ is obtained as

$$
V_{j k}\left(\sin ^{2} 2 \theta, \Delta m^{2}\right)=V_{j k}^{s y s(n e w)}+V_{j k}^{s t a t}\left(\sin ^{2} 2 \theta, \Delta m^{2}\right),
$$

where $V_{j k}^{\text {sys(new) }}$ is the systematic uncertainty for the new data defined as

$$
\mathbf{V}^{\text {sys(new })}=\mathbf{V}^{S B}+\mathbf{V}^{\text {far } / \text { near }}+\mathbf{V}^{\text {det }}+\mathbf{V}^{\text {stab(new })},
$$

and $V_{j k}^{s t a t}\left(\sin ^{2} 2 \theta, \Delta m^{2}\right)$ represents the statistical error defined as,

$$
\mathbf{V}^{\text {stat }}\left(\sin ^{2} 2 \theta, \Delta m^{2}\right)=\left(\begin{array}{ccc}
\frac{1}{M_{1}^{\text {pred }}\left(\sin ^{2} 2 \theta, \Delta m^{2}\right)} & & 0 \\
0 & \ddots & \\
0 & & \frac{1}{M_{16}^{\text {pred }}\left(\sin ^{2} 2 \theta, \Delta m^{2}\right)}
\end{array}\right)
$$

The $\chi^{2}$ for the old data fit is also obtained by substituting $\mathbf{V}^{\text {stab(new) }}$ with $\mathbf{V}^{\text {stab(old) }}$, and changing $M_{j(k)}^{o b s}$ and $M_{j(k)}^{p r e d}\left(\sin ^{2} 2 \theta, \Delta m^{2}\right)$ appropriately.

\subsubsection{New and old data combined fit}

Then, we fit the new and old data sets simultaneously. The $\chi^{2}$ for the combined fit is defined as

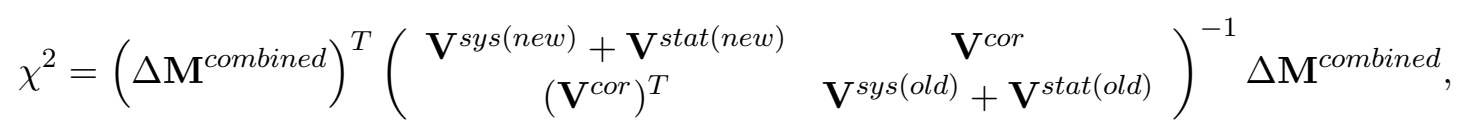

where, $\Delta \mathbf{M}^{\text {combined }}$ is

$$
\Delta \mathbf{M}^{\text {combined }}=\left(\begin{array}{c}
\left(M_{1}^{\text {obs }(\text { new })}-M_{1}^{\text {pred }(\text { new })}\right) / M_{1}^{\text {pred }(\text { new })} \\
\vdots \\
\left(M_{16}^{\text {obs }(\text { new })}-M_{16}^{\text {pred }(\text { new })}\right) / M_{16}^{\text {pred }(\text { new })} \\
\left(M_{1}^{\text {obs (old })}-M_{1}^{\text {pred }(\text { old })}\right) / M_{1}^{\text {pred(old })} \\
\vdots \\
\left(M_{16}^{\text {obs(old })}-M_{16}^{\text {pred(old })}\right) / M_{16}^{\text {pred }(\text { old })}
\end{array}\right)
$$


In this equation, $\mathbf{V}^{\text {sys(new) }}$ and $\mathbf{V}^{\text {sys(old) }}$ represent systematic uncertainties for new and old data calculated by Eq. (7.21), and $\mathbf{V}^{\text {stat(new) }}$ and $\mathbf{V}^{\text {stat(old) }}$ are statistical uncertainties defined by Eq. (7.22). We assume that all uncertainties except for the data stability and statistical uncertainties are $100 \%$ correlated between the new and old data sets. Hence, the correlation term, $\mathbf{V}^{c o r}$, is calculated as

$$
\mathbf{V}^{\text {cor }}=\mathbf{V}^{S B}+\mathbf{V}^{\text {far/near }}+\mathbf{V}^{\text {det }}
$$

which is the all systematic uncertainties except for the stability error defined in Sec. 7.4.2.

\subsubsection{Confidence level determination}

We compute the confidence level (C.L.) of the oscillation hypotheses by $\Delta \chi^{2}\left(\sin ^{2} 2 \theta, \Delta m^{2}\right)=$ $\chi^{2}\left(\sin ^{2} 2 \theta, \Delta m^{2}\right)-\chi^{2}$ (best) values, where $\chi^{2}\left(\sin ^{2} 2 \theta, \Delta m^{2}\right)$ is the $\chi^{2}$ at the oscillation prediction being tested, and $\chi^{2}$ (best) is the smallest $\chi^{2}$ value in the $\left(\sin ^{2} 2 \theta, \Delta m^{2}\right)$ plane. Then, an allowed region can be defined by a single number, $\Delta \chi_{c}^{2}\left(\sin ^{2} 2 \theta, \Delta m^{2}\right)$, as

$$
\Delta \chi^{2}\left(\sin ^{2} 2 \theta, \Delta m^{2}\right)<\Delta \chi_{c}^{2}\left(\sin ^{2} 2 \theta, \Delta m^{2}\right) .
$$

Assuming that the probability density function is a two-dimensional Gaussian shape on the $\left(\sin ^{2} 2 \theta, \Delta m^{2}\right)$ plane, the constant value of $\Delta \chi_{c}^{2}$ is 4.61 for $90 \%$ C.L.

However, this assumption is not applicable in this analysis because of the sinusoidal nature of the oscillation function (Eq. (7.14)). This is especially true in the case that no significant oscillation signal is observed, and an allowed region spans for wide range of $\Delta m^{2}$ region 11 . Hence, we use Feldman-Cousins' method [109] to obtain the confidence level at each oscillation parameter point $\left(\sin ^{2} 2 \theta, \Delta m^{2}\right)$. In this method, 1000 "fake-data" predictions are formed, using random draws of the statistical and systematic uncertainties with oscillation hypothesis of $\left(\sin ^{2} 2 \theta, \Delta m^{2}\right)$. Figure 7.13 show the some example of the fits to the fake data sets with null oscillation for the "new" data set. The $\chi^{2}$ values for the null and best fit oscillation points are shown in Figure 7.14. The mean of the reduced $\chi^{2}$ for null oscillation is $15.83 /(16 \mathrm{DOF})$ and is consistent to 1 , which verifies the variation of fake data sets.

Then, we obtain the distribution of $\Delta \chi^{2}=\chi^{2}(0,0)-\chi^{2}$ (best) as shown in Figure 7.15 , by which we can calculate the probability density function of the $\chi^{2}, P\left(\Delta \chi^{2}\right)$. The $\Delta \chi^{2}$ value corresponds to $90 \% \mathrm{CL}\left(\Delta \chi_{90}^{2}\right)$ is obtained from the relation:

$$
\frac{\int_{0}^{\Delta \chi_{90}^{2}} P(x) d x}{\int_{0}^{\infty} P(x) d x}=0.9 .
$$

We repeat this process for each set of $\left(\sin ^{2} 2 \theta, \Delta m^{2}\right)$ true oscillation parameter being tested. Then, we obtain the $\Delta \chi^{2}$ values required to allow or exclude the oscillation hypothesis by $90 \%$ C.L. $\left(\Delta \chi_{90}^{2}\right)$ at each $\left(\sin ^{2} 2 \theta, \Delta m^{2}\right)$ value. The values of $\Delta \chi_{90}^{2}$ at each oscillation parameter are shown in Fig. 7.16,

\subsection{Sensitivity}

The sensitivity is defined as the average of limits obtained from fake experiments with null oscillation. Figure 7.17 shows the $90 \%$ CL. sensitivity for the $\nu_{\mu}$ disappearance. The expected $\pm 1 \sigma$ band, which is defined as the region contain the $67 \%$ of limits from fake experiments, is also shown in the plot. Sensitivities for $95 \%$ and $99 \%$ C.L. are also shown in Figure 7.18, The expected sensitivity is improved from the MiniBooNE only $\nu_{\mu}$ disappearance result, since the flux and cross section uncertainties have been substantially reduced by the SciBooNE measurements. We achieved the world best sensitivity for the $\nu_{\mu}$ disappearance at $0.5<\Delta m^{2}<30\left(\mathrm{eV}^{2}\right)$.

\footnotetext{
${ }^{1}$ In Feldman-Cousins' paper [109]: For high values of $\Delta m^{2}$ fluctuations can cause a global minimum in a "wrong" trough of the function, increasing the value of $\Delta \chi^{2}$ from what it would be if there were only one trough.
} 

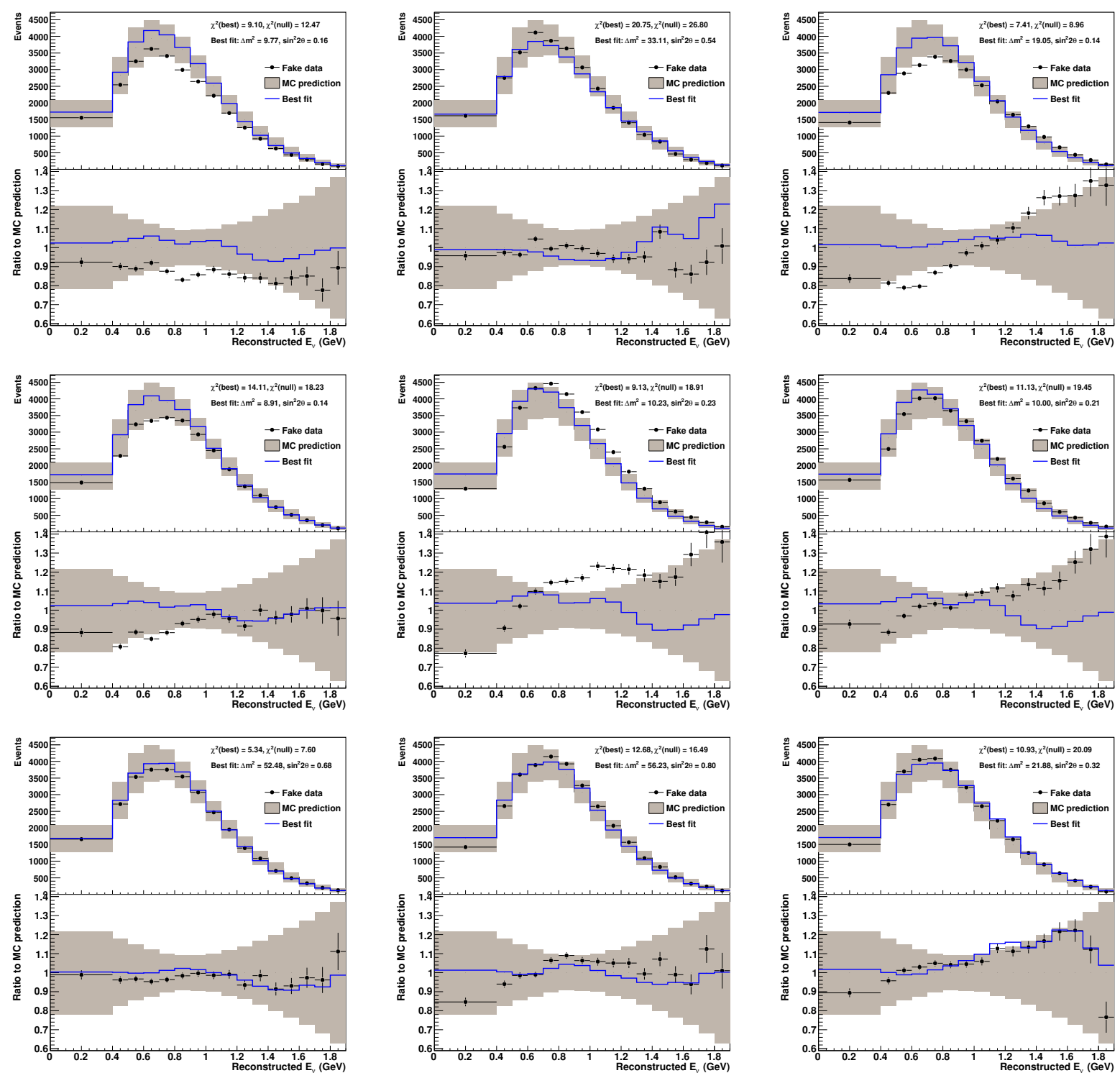

Figure 7.13: Examples of fits to the fake data sets randomly drown from the total systematic and statistical uncertainties for the new data. The shaded region show the MC prediction with the systematic uncertainty. The points show the fake data and the blue curves show the best fit to the fake data. 

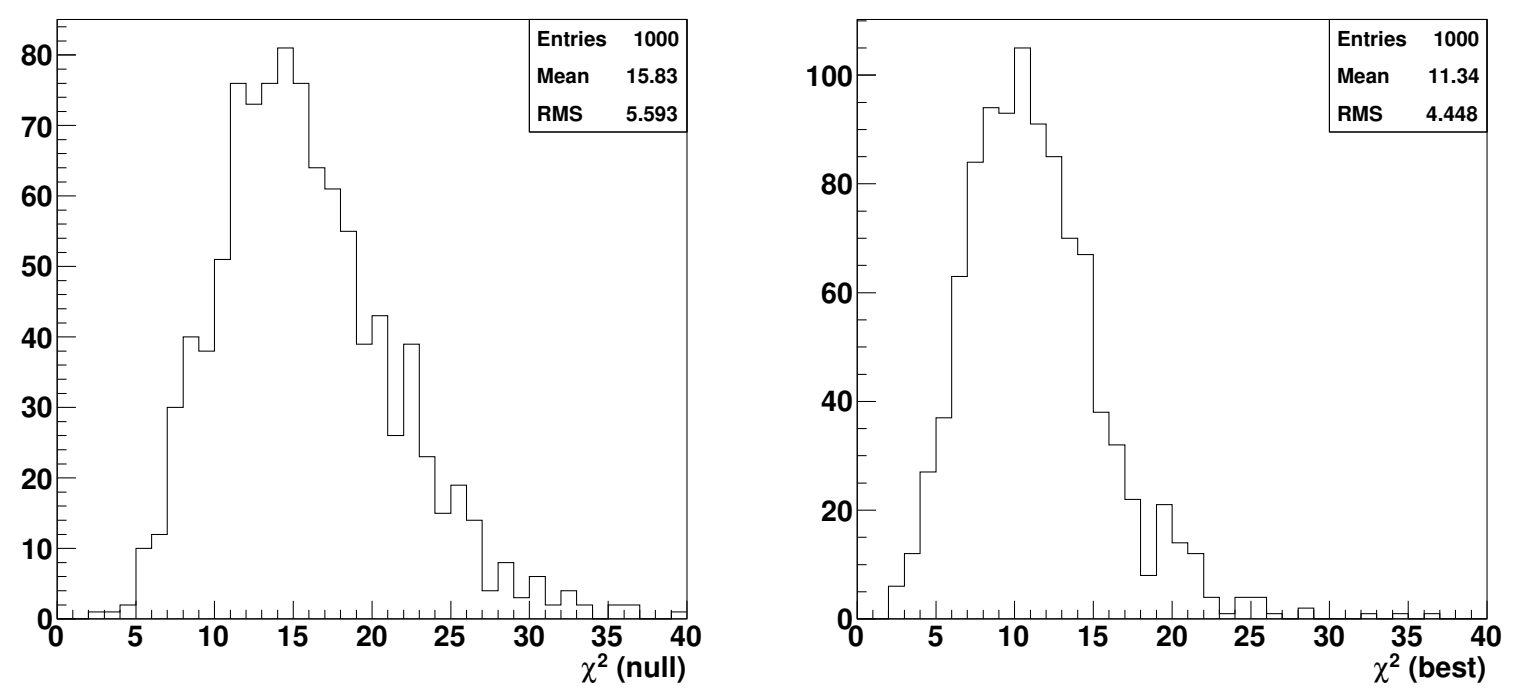

Figure 7.14: Distributions of the (left) $\chi^{2}(0,0)$ and (right) $\chi^{2}$ (best) for fits to 1000 fake data sets (fake experiments) with null oscillation.

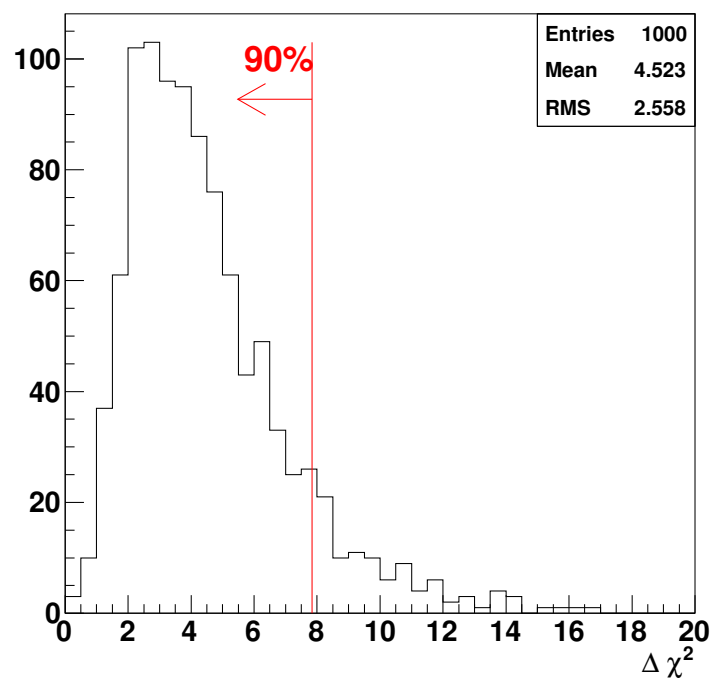

Figure 7.15: Distribution of the $\Delta \chi^{2}=\chi^{2}(0,0)-\chi^{2}$ (best) values for fits to 1000 fake data sets (fake experiments) with null oscillation hypothesis. The vertical red line indicate the $\Delta \chi^{2}$ value which contain $90 \%$ of fake experiments below that. 

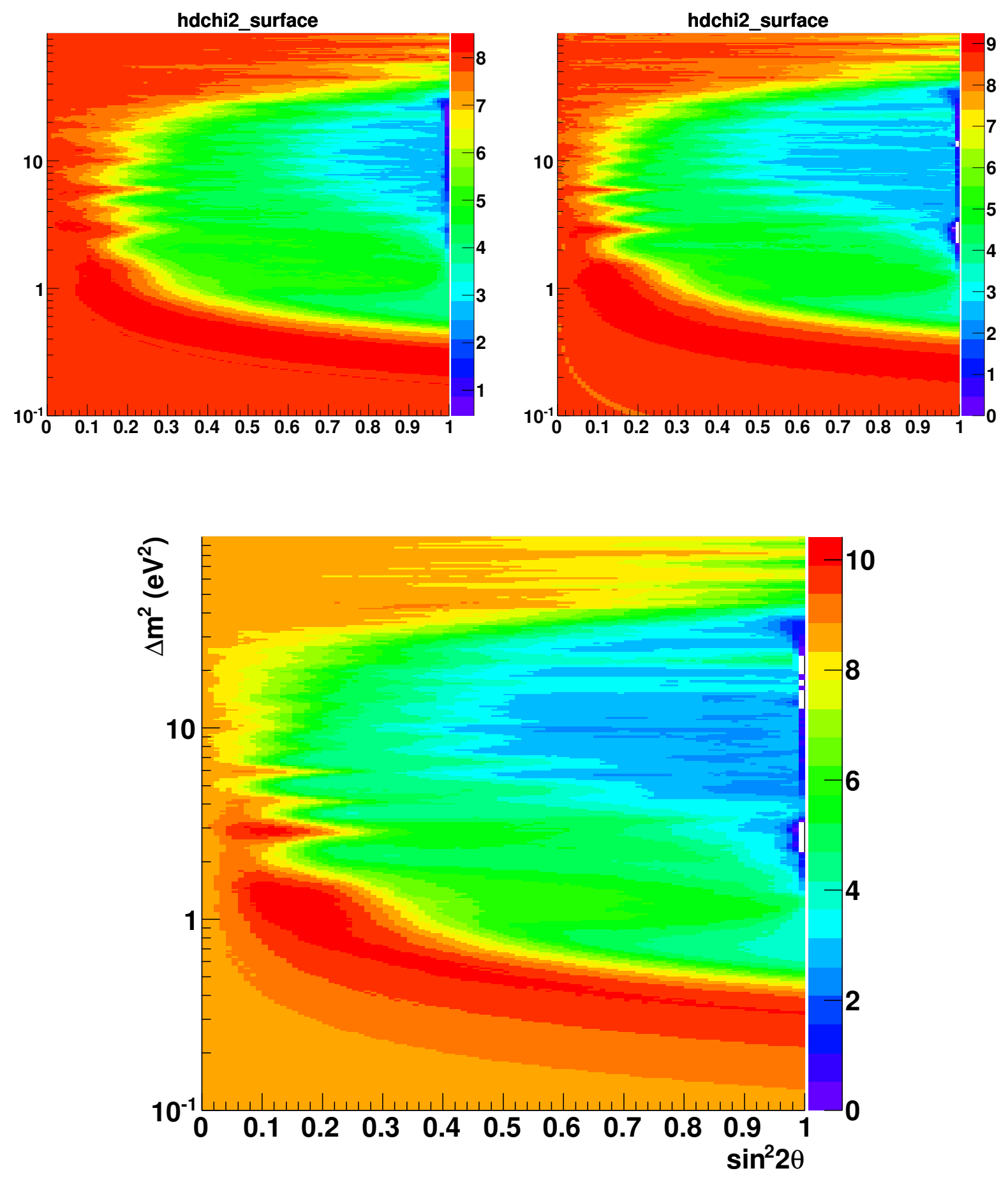

Figure 7.16: $\Delta \chi^{2}$ values required to allow or exclude the oscillation hypothesis by $90 \%$ C.L. $\left(\Delta \chi_{90}^{2}\right)$ at each $\left(\sin ^{2} 2 \theta, \Delta m^{2}\right)$ for the new data (top left), old data (top right), and combined data (bottom) fits. 


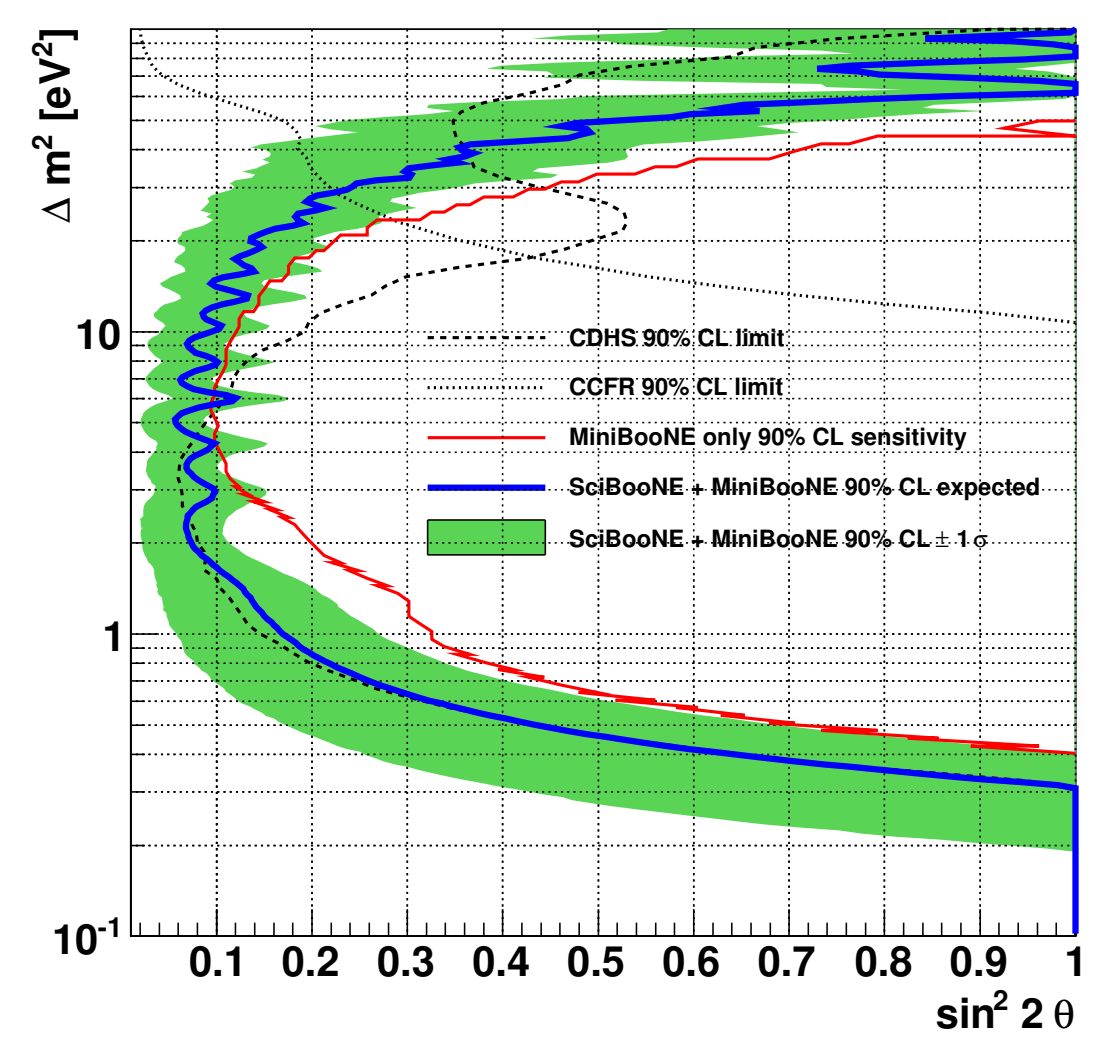

Figure 7.17: The sensitivity for $\nu_{\mu}$ disappearance. The thick solid curve and the filled region are the $90 \%$ CL sensitivity and $\pm 1 \sigma$ band from the SciBooNE-MiniBooNE joint analysis, respectively. The thin solid curve is the MiniBooNE-only 90\% CL sensitivity. The 90\% CL limits from CDHS [36] and CCFR 35] experiments are also shown.

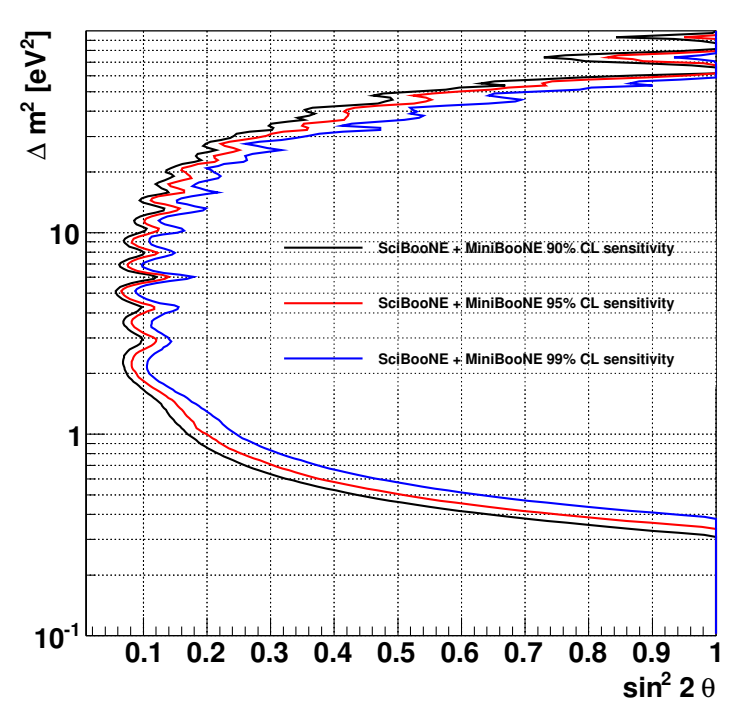

Figure 7.18: $90 \%, 95 \%$ and $99 \%$ C.L. sensitivities to $\nu_{\mu}$ dieappearanfce for the combined data fit. 


\subsection{Result and discussions}

\subsection{1 $\nu_{\mu}$ disappearance search result}

Figure 7.19 shows the MiniBooNE data distribution compared with the MC prediction with the null, best fit, and several other parameter sets. We find the best fit point at $\left(\sin ^{2} 2 \theta, \Delta m^{2}\right)=$ $(0.51,41.7)$ with $\chi^{2}$ (best) $=35.6 / 30(D O F)$. The $\chi^{2}$ value for the null oscillation prediction is $\chi^{2}($ null $)=41.5 / 32(D O F)$. The value of $\Delta \chi^{2}=\chi^{2}(0,0)-\chi^{2}$ (best) is 5.9, while the $\Delta \chi^{2}$ value for the $90 \%$ confidence level at null oscillation point, $\Delta \chi_{90}^{2}(0,0)$, is estimated to be 8.41 . Hence, the observed best fit point is consistent with the null oscillation hypothesis at $90 \%$ C.L., and we found no significant oscillation signal. Although there is a data deficit, the best fit oscillation doesn't well reproduce $E_{\nu}^{R e c}$ distribution and the data-MC difference is covered by the systematic uncertainty. Therefore, the significance is small.

Figure 7.20 show the obtained $90 \%$ CL limit in the $\left(\sin ^{2} 2 \theta, \Delta m^{2}\right)$ plane, compared with the sensitivity and other previous measurements. The same plot with logarithm $\sin ^{2} 2 \theta$ scale is shown in Figure 7.21. The limits for other confidence levels are shown in Figure 7.22, In this figure, the best fit point, and the four oscillation parameter points shown in Figure 7.19 are also shown.

In general, the obtained $90 \%$ C.L. limit is consistent with the expected $\pm 1 \sigma$ sensitivity region, which also indicates the observed data is consistent with the null oscillation hypothesis. At $10<$ $\Delta m^{2}<30\left(\mathrm{eV}^{2}\right)$, we set the world best limit for the $\nu_{\mu}$ disappearance. Hence, this is the strongest constraint for the sterile neutrino mixing at this $\Delta m^{2}$ region. This result can also constrain the parameter spaces for $(3+2)$ sterile neutrino model [27 31], and many other exotic scenarios [40 42].

At the low $\Delta m^{2}$ region below $3.0 \mathrm{eV}^{2}$, the limits are worse than the sensitivity. This is because we observed a data deficit similar to the oscillation signal with these $\Delta m^{2}$ values at low $E_{\nu}^{R e c}$ region, as shown in Figure 7.19. However, as described before, this data deficit is covered by the current systematic uncertainties and also the prediction doesn't reproduce the data for the entire $E_{\nu}^{R e c}$ region. Hence, significance of the oscillation hypothesis is small.

\subsubsection{Possible improvements}

To improve the sensitivity further and to understand the observed data deficit, the reduction of the systematic errors is essential. As shown in Figure 7.8, the dominant errors of this $\nu_{\mu}$ disappearance analysis come from the uncertainties of the neutrino interaction cross sections and the MiniBooNE detector responses. With further understanding of the neutrino-nucleus interaction cross sections by the SciBooNE measurements and future experiments, will can reduce these errors and achieve higher sensitivity.

\subsubsection{Muon anti-neutrino disappearance}

Although it is not the topic of this thesis, searching for muon anti-neutrino dieappearance is also an important subject. If there is some mechanism which violates $\mathrm{CP}$ or $\mathrm{CPT}$ invariance, $P\left(\nu_{\alpha} \rightarrow \nu_{\beta}\right)$ is different from $P\left(\bar{\nu}_{\alpha} \rightarrow \bar{\nu}_{\beta}\right)$. This possibility become particularly interesting since MiniBooNE observed an event excess in the $\bar{\nu}_{\mu} \rightarrow \bar{\nu}_{e}$ search [26] while they find null oscillation signal in the $\nu_{\mu} \rightarrow \nu_{e}$ search [24, 25]. Hence, it is increasingly important to test neutrino and anti-neutrino oscillation independently.

If we take anti neutrino results only, there is no short-baseline $\nu_{\mu}$ disappearance measurement sensitive to $\Delta m^{2} \sim 1 \mathrm{eV}^{2}$, except for the recent MiniBooNE only measurement [44. Also, as shown in Figure 1.6 a global $(3+1)$ sterile neutrino fit anti-neutrino data only suggests a significant $\left(\sin ^{2} 2 \theta>0.2\right)$ short-baseline $\bar{\nu}_{\mu}$ disappearance [29, 45]. This further motivate to search for $\bar{\nu}_{\mu}$ disappearance in this region.

As mentioned in Sec. 3.2 , we also took beam data in anti-neutrino mode both at SciBooNE and at MiniBooNE. A SciBooNE-MiniBooNE joint $\bar{\nu}$ disappearance analysis is now in progress. 
$\longrightarrow$ Data

Null oscillation

\section{Best fit}
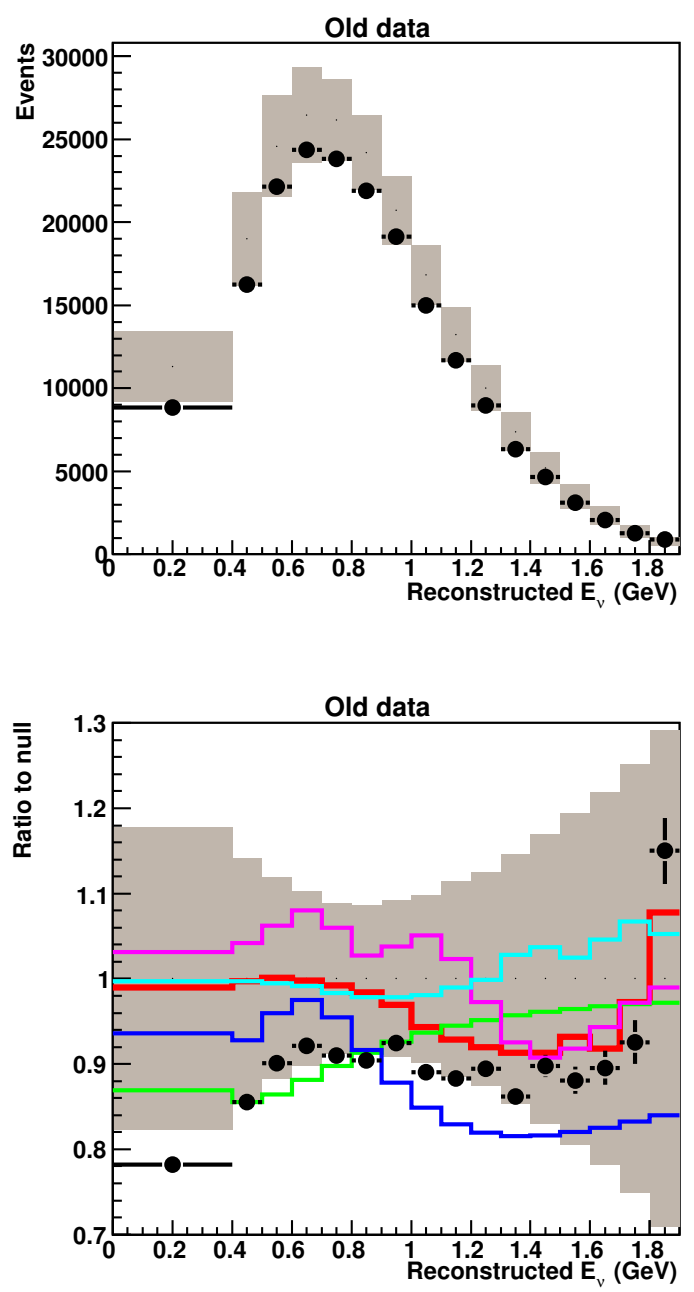

$\Delta M^{2}=1.00, \sin ^{2} 2 \theta=0.20$

$\Delta M^{2}=3.16, \sin ^{2} 2 \theta=0.20$

$\Delta M^{2}=10.00, \sin ^{2} 2 \theta=0.20$

$\Delta M^{2}=31.62, \sin ^{2} 2 \theta=0.20$
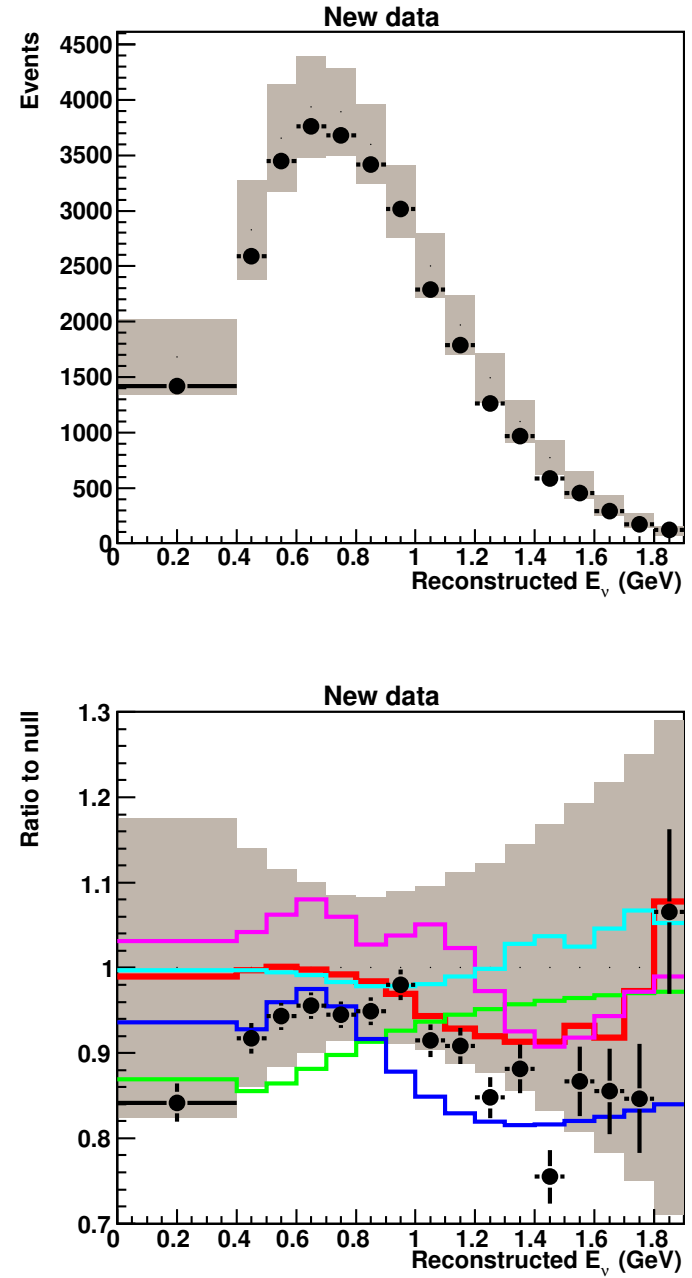

Figure 7.19: The distributions of the reconstructed $E_{\nu}$ for the MiniBooNE old (left) and new (right) data compared with the MC predictions. The top panels show the number of events and the bottom plots shows the ratio to the MC prediction with null oscillation. The prediction of the best fit $\left(\sin ^{2} 2 \theta, \Delta m^{2}\right)$ and several other parameter sets are also shown in the bottom panels. 


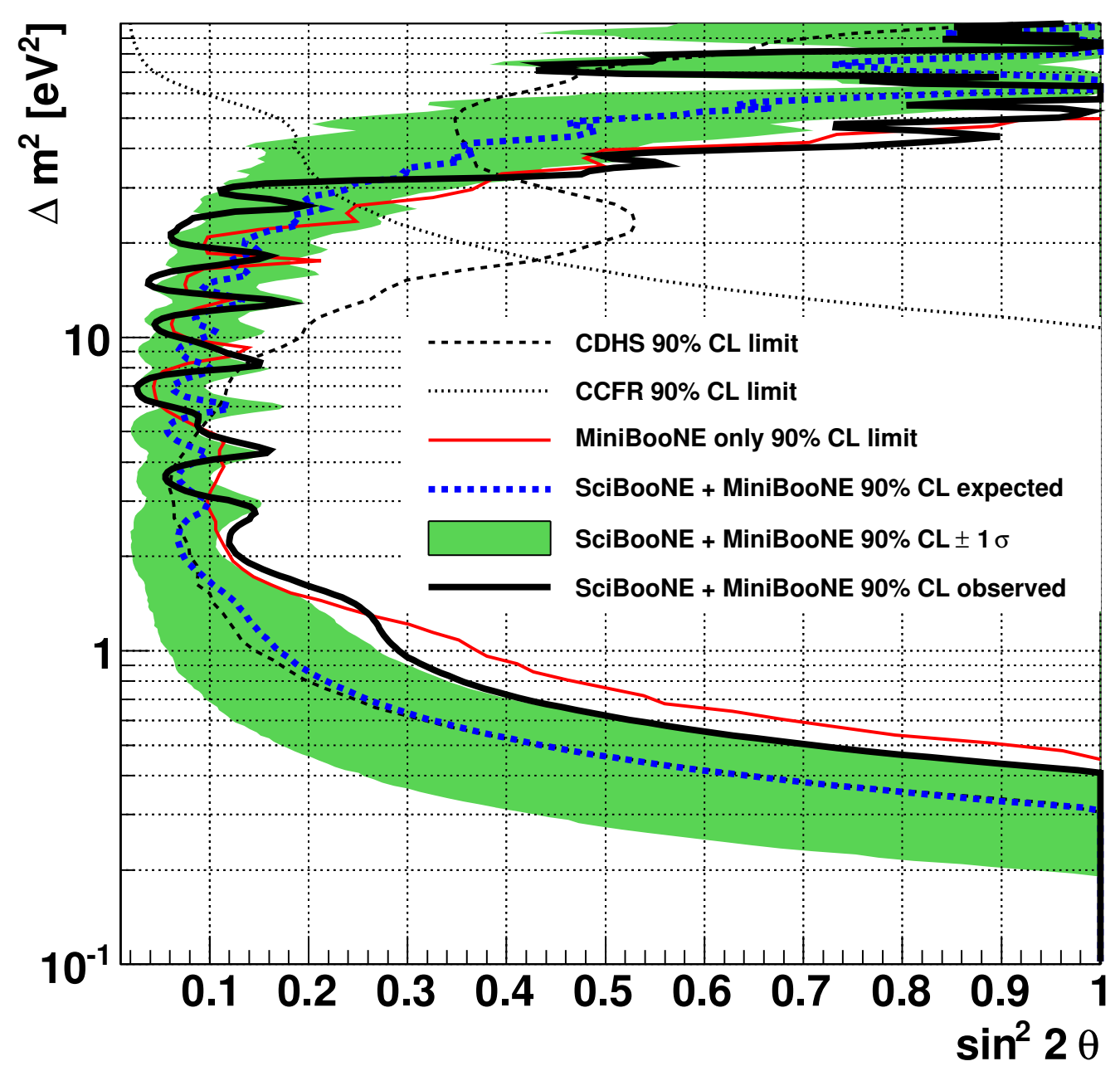

Figure 7.20: The 90\% CL limit for the data fit. Oscillation parameters at the right-hand side of the limit curve are excluded. The thick solid curve and the filled region are the 90\% CL sensitivity and $\pm 1 \sigma$ band from SciBooNE-MiniBooNE joint analysis, respectively. The thin solid curve is the MiniBooNE-only 90\% CL sensitivity. The 90\% CL limits from CDHS [36] and CCFR [35] experiments are also shown. 


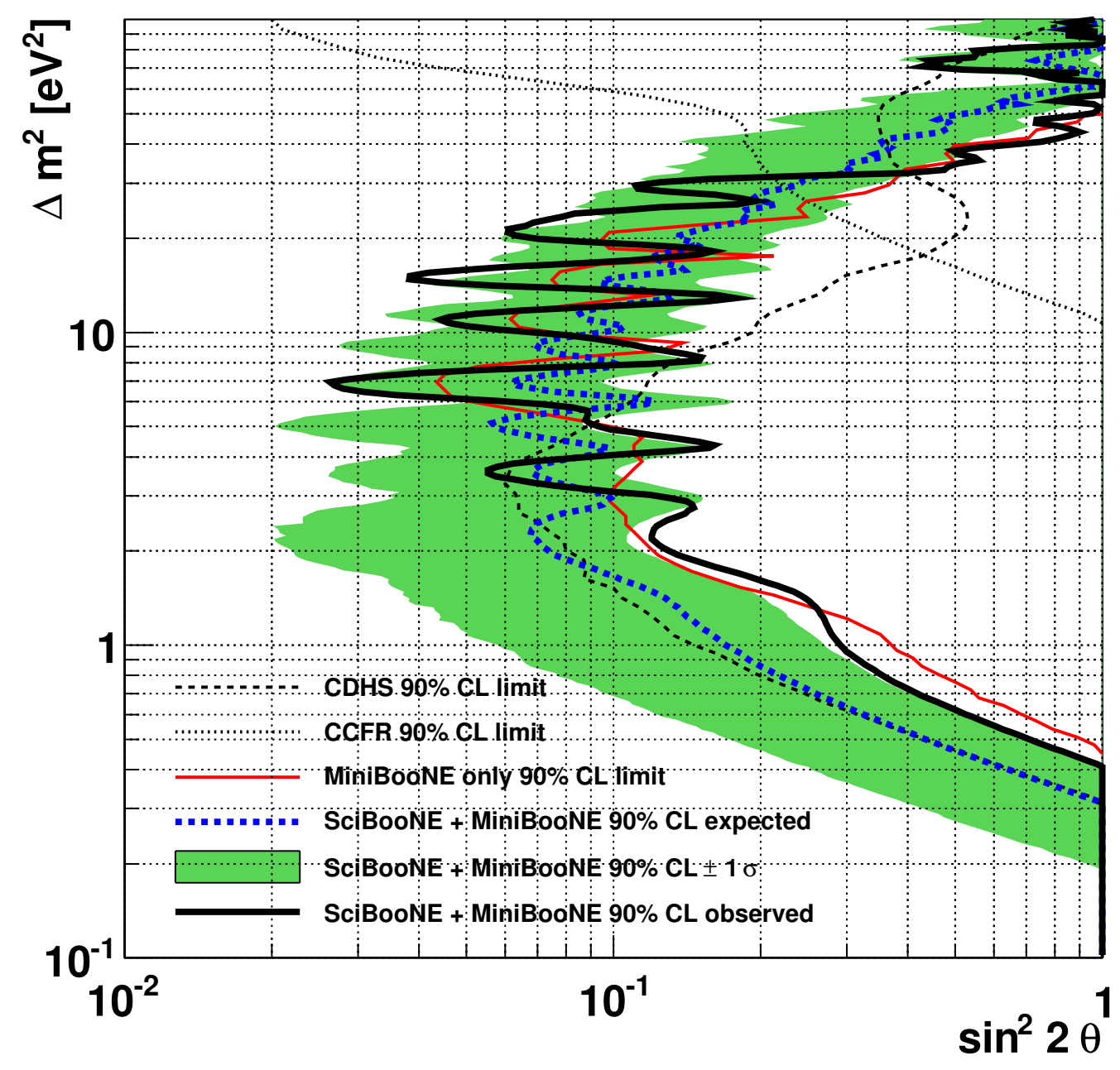

Figure 7.21: Figure 7.20 shown with logarithm $\sin ^{2} 2 \theta$ scale. 


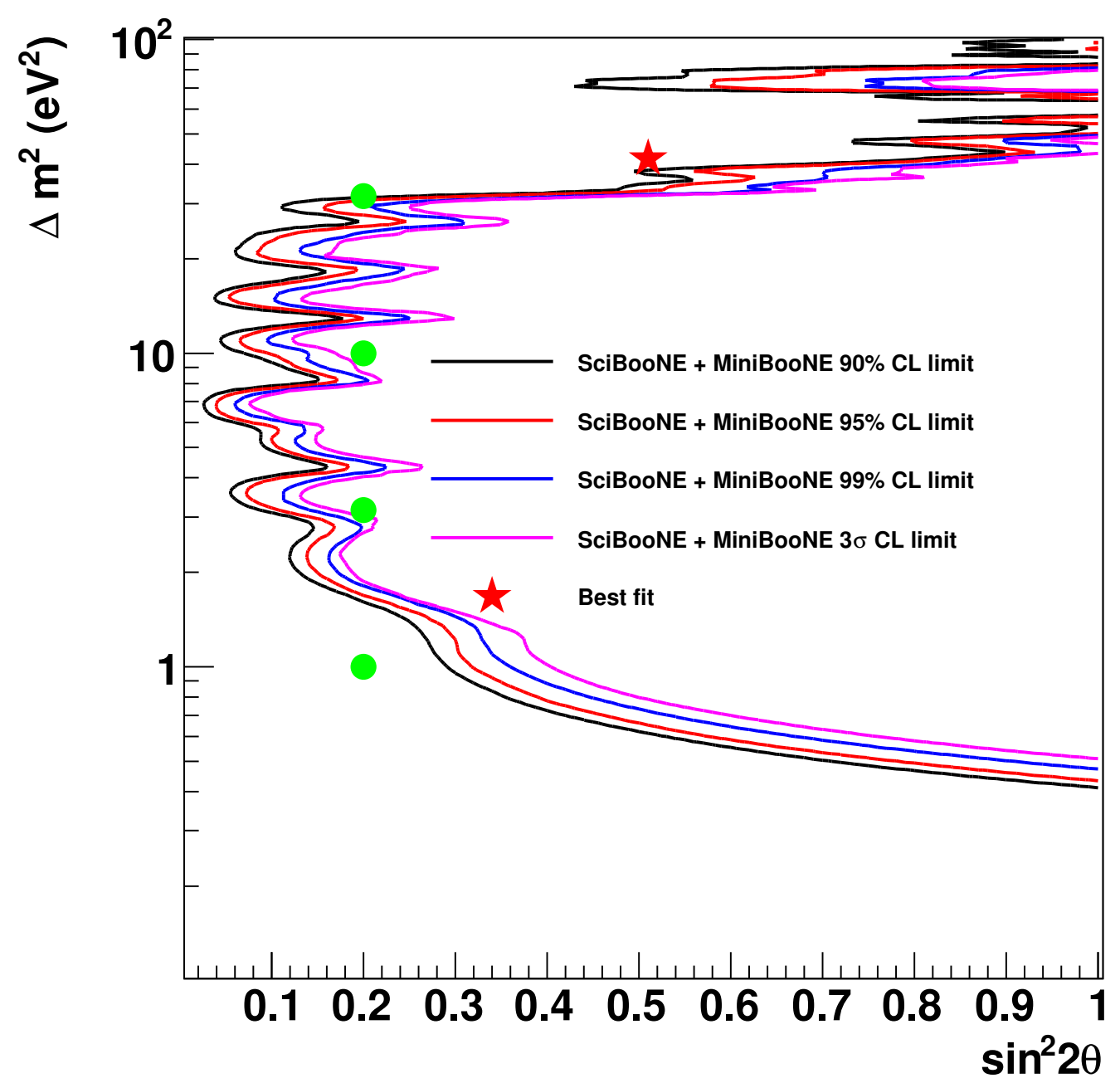

Figure 7.22: The data fit limits with $90 \%, 95 \%, 99 \%$ and $3 \sigma$ confidence levels. The best fit point is indicated by the red star. The four green dots indicate the oscillation parameters shown in Figure 7.19 
The event selection, fit method and the error analysis developed for this $\nu_{\mu}$ disappearance analysis are directly applicable for the $\bar{\nu}_{\mu}$ analysis as well. In addition, it is important to constrain the contamination of "wrong-sign" (neutrino) backgrounds, which is expected to be more than $30 \%$ in anti-neutrino mode, as shown in Figure 7.23. The limit from this $\nu_{\mu}$ disappearance search provides an essential information for anti-neutrino disappearance analysis, by constraining the oscillation effect of the "wrong-sign" events. Then, we will achieve a higher sensitivity than the previous $\bar{\nu}_{\mu}$ disappearances search.
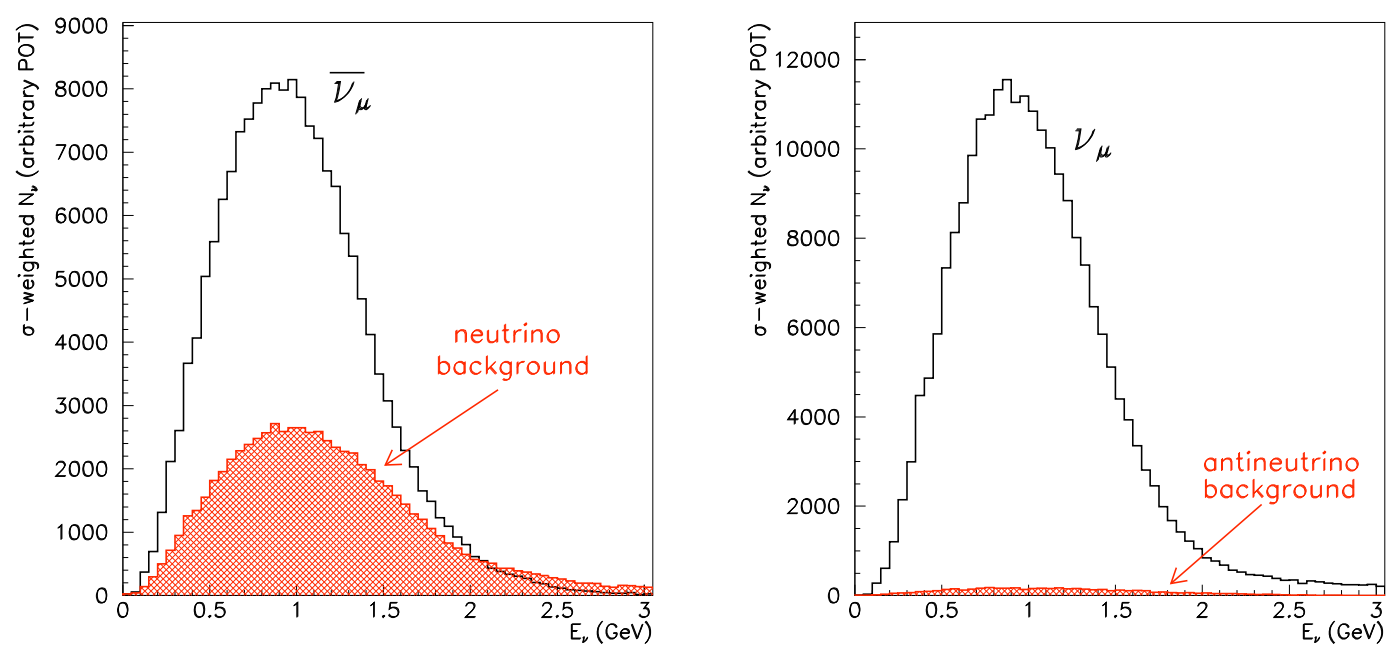

Figure 7.23: Expected neutrino energy spectrum for right-sign and wrong-sing neutrino events (after applying cross-section) in anti-neutrino (left) and neutrino (right) mode running. The wrong sign events are shown with the hatched histograms. Plots are taken from Ref. [46].

\subsection{Summary}

In summary, we presented an improved search for muon neutrino disappearance at the Fermilab Booster Neutrino Beam, by using the SciBooNE measurement to improve the uncertainties of the neutrino flux and cross section, while the previous measurements by MiniBooNE only are limited by those flux and cross section uncertainties. We developed a method to constrain the MiniBooNE distribution using the result of CC interaction measurements at SciBooNE (Sec. 6), and produced a reliable prediction of the MiniBooNE distribution with smaller systematic uncertainties. We succeeded to reduce the systematic errors of flux and cross section uncertainties to the same level of the MiniBooNE detector uncertainties. The resulting sensitivity supersedes the previous result, and we achieved the world best sensitivity for the $\nu_{\mu}$ disappearance at $0.5<\Delta m^{2}<30\left(\mathrm{eV}^{2}\right)$.

Comparing the prediction with the observation, we found no significant oscillation signal, and set an upper limit in the $\left(\sin ^{2} 2 \theta, \Delta m^{2}\right)$ plane. At $10<\Delta m^{2}<30\left(\mathrm{eV}^{2}\right)$, we set the world best limit for the $\nu_{\mu}$ disappearance. Hence, this is the strongest constraint for the sterile neutrino mixing at this $\Delta m^{2}$ region. This result can also constrain the parameter spaces for $(3+2)$ sterile neutrino model [27-31], and many other exotic scenarios [40 42]. At the low $\Delta m^{2}$ region below $3.0 \mathrm{eV}^{2}$, the limits are worse than the sensitivity because of the deficit of events compared to the MC prediction.

To improve the sensitivity further, reductions of the cross section and MiniBooNE detector uncertainties are essential. With further understanding of the neutrino-nucleus interaction cross sections by the SciBooNE measurements and future experiments, we will be able to reduce these errors and achieve higher sensitivity. There is also a proposal for a second MiniBooNE-type detector on the BNB [110], which will cancel the detector uncertainty as well.

Finally, a SciBooNE-MiniBooNE joint muon anti-neutrino disappearance analysis is also ongo- 
ing. The methods developed for this $\nu_{\mu}$ disappearance analysis are directly applicable for the $\bar{\nu}_{\mu}$ analysis as well. Also this result can be used to constrain the wrong-sign events in anti-neutrino mode. Then, we will achieve a higher sensitivity than the previous $\bar{\nu}_{\mu}$ disappearances search [44]. 


\section{Chapter 8}

\section{Conclusions}

In this thesis, we reported on a measurement of muon neutrino charged current inclusive interactions on carbon in the few $\mathrm{GeV}$ region, using the Fermilab Booster Neutrino Beam.

We collected high-statistics CC interaction sample at SciBooNE, and extracted energy dependent inclusive charged current interaction rates and cross sections for a wide energy range from $0.25 \mathrm{GeV}$ to $\sim 3 \mathrm{GeV}$. We measure the interaction rates with $6-15 \%$ precision, and the cross sections with 10-30\% precision. We also made an energy integrated measurements, with the precisions of $3 \%$ for the rate, and $8 \%$ for the cross section measurements. This is the first measurement of the $\mathrm{CC}$ inclusive cross section on carbon around $1 \mathrm{GeV}$. The results have following three impacts.

First, these cross section results are essential inputs for the current and future neutrino oscillation experiments which uses neutrino beam around $1 \mathrm{GeV}$, such as MiniBooNE, T2K and $\mathrm{NO} \nu \mathrm{A}$. This inclusive interaction measurement is nearly free from effects of hadron re-interactions in the nucleus, and hence it is complementary to other exclusive cross section measurements. The another unique feature of this measurement is that the result covers a wide energy range from $0.25 \mathrm{GeV}$ to $\sim 3 \mathrm{GeV}$. Hence, this is a essential measurement to test the consistency between recent MiniBooNE measurements below $2 \mathrm{GeV}$ and the higher energy measurements by NOMAD and MINOS above $\sim 3 \mathrm{GeV}$.

For neutrino interaction simulation, we use the two simulators, NEUT and NUANCE, which are both commonly used in recent neutrino oscillation experiments. We made a detailed comparison of these simulators with our measurements, and described the difference with these. This directly helps tuning the cross section models in each simulators, and therefor help reducing cross section uncertainties for the future oscillation measurements, such as T2K.

Second, this analysis provides the normalization for SciBooNE's previous cross section ratio measurements [68 70], which are important to understand the backgrounds for $\nu_{\mu}$ disappearance and $\nu_{e}$ appearance measurements. We extracted CC interaction cross sections with the same definition used in the previous analyses, and computed the absolute cross sections of these interaction modes. Then, precise comparison with various cross section models become possible, and again, it will help MiniBooNE, T2K and $\mathrm{NO} \nu \mathrm{A}$.

Third, the result of the rate measurement is used to constrain the product of the neutrino flux and the cross section for MiniBooNE. We conducted a search for short-baseline muon neutrino disappearance using data both from SciBooNE and MiniBooNE, to test a possible neutrino oscillation with sterile neutrinos which suggested by the LSND experiment. With this constraint by SciBooNE, we significantly reduced the flux and the cross section uncertainties at MiniBooNE, and achieved the world best sensitivity for the $\nu_{\mu}$ disappearance at $0.5<\Delta m^{2}<30\left(\mathrm{eV}^{2}\right)$. We found no significant oscillation signal, and set one the world strongest limits at $10<\Delta m^{2}<30\left(\mathrm{eV}^{2}\right)$, which further constrain the sterile neutrino models.

For this oscillation analysis, the dominant systematic uncertainties are the cross section and the MiniBooNE detector uncertainties. Hence, to improve the sensitivity further, reductions of these errors are essential. With further precise measurements of the neutrino-nucleus interaction cross 
sections by SciBooNE and other future experiments, we will be able to reduce these errors and achieve higher sensitivity.

Finally, a SciBooNE-MiniBooNE joint muon anti-neutrino disappearance analysis is also ongoing. The methods developed for this $\nu_{\mu}$ disappearance analysis are directly applicable for the $\bar{\nu}_{\mu}$ analysis as well. Also this result can be used to constrain the wrong-sign events in anti-neutrino mode. Then, we will achieve a higher sensitivity than the previous $\bar{\nu}_{\mu}$ disappearances search [44]. 


\section{Appendix A}

\section{Study of the spectrum fit methods}

\section{A.1 Choice of samples and binning}

\section{A.1.1 Energy resolution}

We reconstruct neutrino spectrum using muon kinematics only. Therefore, the neutrino energy resolutions are determined by two sources: (1) resolutions of the muon momentum and angle, and (2) contamination of different interaction modes.

The resolutions of muon momentum and angle are shown in Sec. 5.1.4. Here we discuss the effect of the source (2).

Since the dominant CC interaction mode is CCQE in our energy region, we discuss the effect of contamination of different interaction modes by comparing true $E_{\nu}$ and $E_{\nu}^{Q E}$.

Figure A.1 shows the difference of $E_{\nu}$ and $E_{\nu}^{Q E}$ from MRD-stopped sample for each true $E_{\nu}$ regions. We can see that as the energy increases, the QE contamination decreases. There are 2 reasons which explain this effect. First, the cross section ratio of CCQE/CC-inclusive decreases as energy increases. Second, given the same energy neutrino, muons from CCQE interaction tend to have larger momentum than any other CC interaction. Since these muons are less likely to stop in the MRD, we loose acceptance for CCQE event from high energy neutrinos.

Figure A.2 is showing the mean reconstructed neutrino energy and its spread as a function of the true energy. We basically loose the neutrino energy resolution at $E_{\nu}>\sim 1.3 \mathrm{GeV}$ for MRDstopped sample. Hence, the additional information from the MRD-penetrated events can help understanding the spectrum at the high energy region.

\section{A.1.2 Acceptance comparison to MiniBooNE}

Figures A.3, A.4 and A.5 shows the distributions of true muon kinematics and neutrino energy of the accepted events at SciBooNE and MiniBooNE.

We can see the SciBar-stopped + MRD-stopped sample gives us similar muon momentum acceptance to MiniBooNE. On the other hand, MRD-stopped and MRD-penetrated samples have biased acceptance to forward going muons, since we require muon to be reached to MRD.

In terms of neutrino energy, the sum of SciBar-stopped and MRD-stopped samples provides similar coverage to MiniBooNE, and the acceptance of MRD-penetrated sample tend to have higher energy than MiniBooNE sample.

\section{A.1.3 Sample and binning}

To summarize the discussion above, the sum of SciBar-stopped and MRD-stopped sample provide most similar acceptance to MiniBooNE. However, MRD-stopped sample has very poor energy resolution at the $E_{\nu}>\sim 1.3 \mathrm{GeV}$. This situation is expected be improved by adding MRD-penetrated sample, which provides the flux at the highest energy region. 

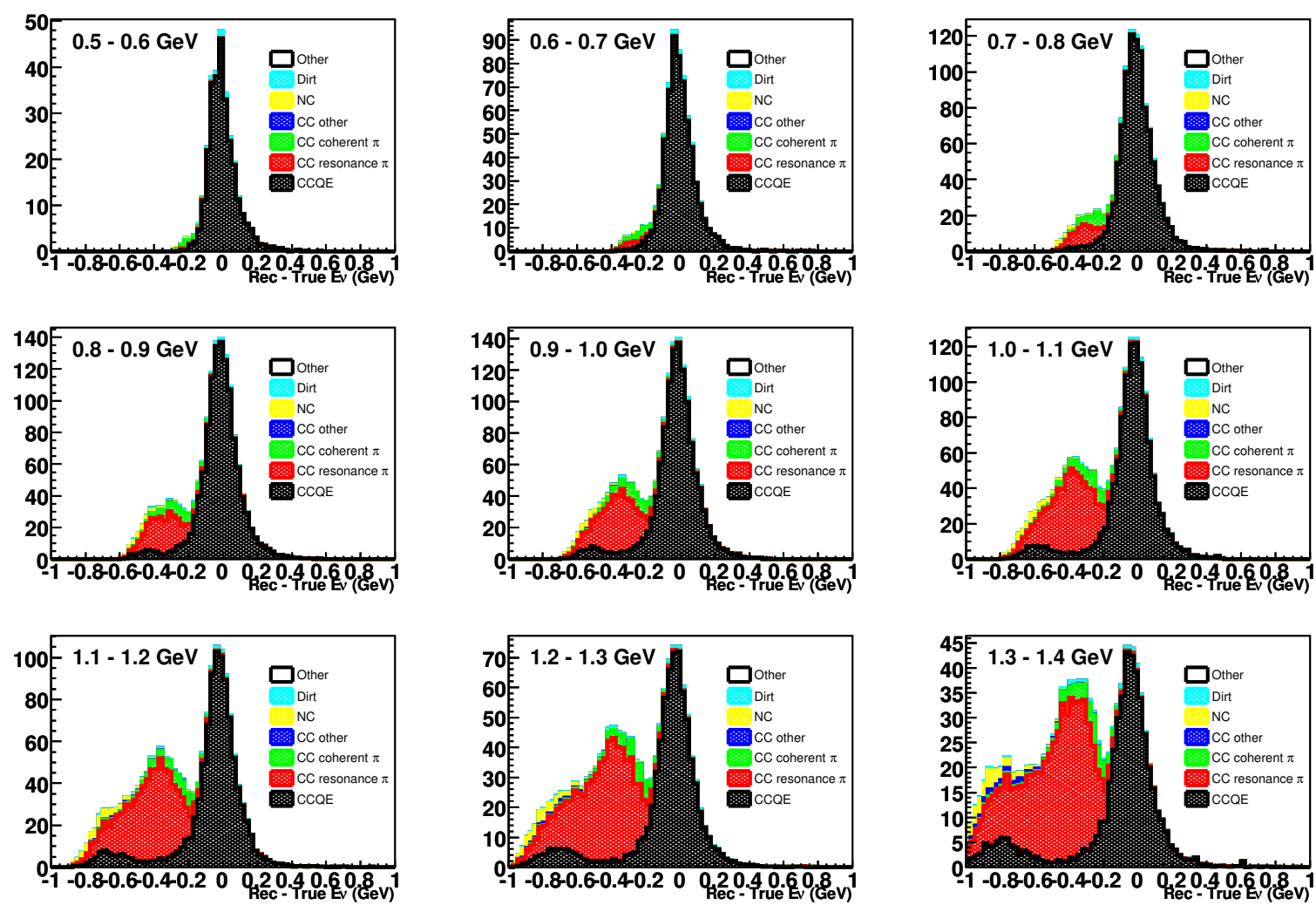

Figure A.1: Neutrino energy resolutions for each true $E_{\nu}$ regions for MRD-stopped events.

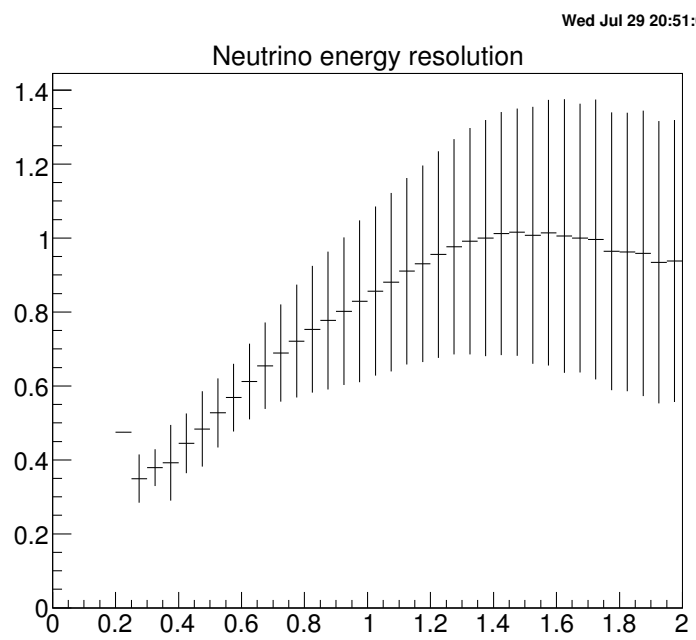

Figure A.2: Mean value of reconstructed energy assuming CCQE kinematics $\left(E_{\nu}^{Q E}\right)$, as a function of true $E_{\nu}$. The error bars represents the RMS of $E_{\nu}^{Q E}$ distribution. 

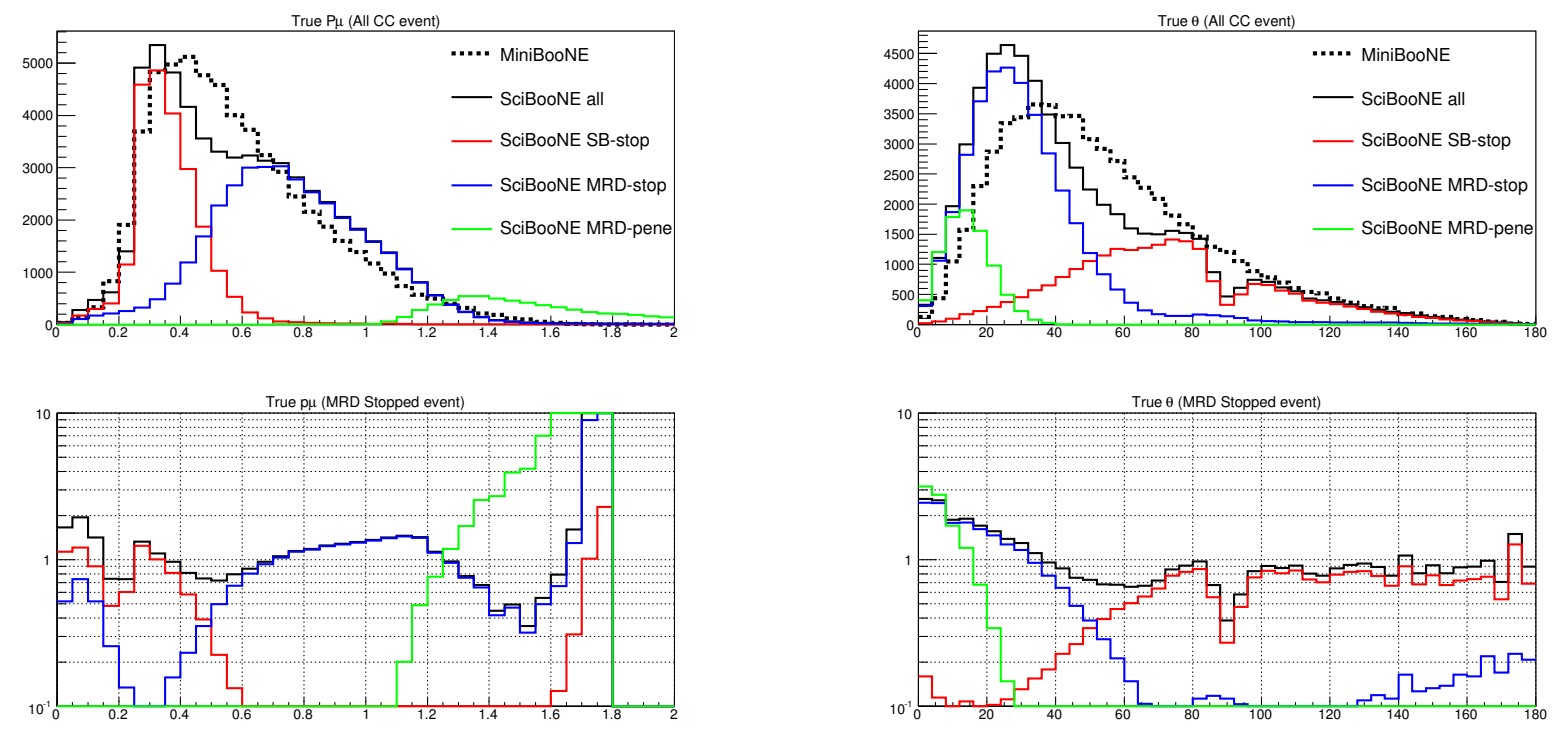

Figure A.3: Comparison of true $p_{\mu}$ distributions of selected sample at SciBooNE and MiniBooNE
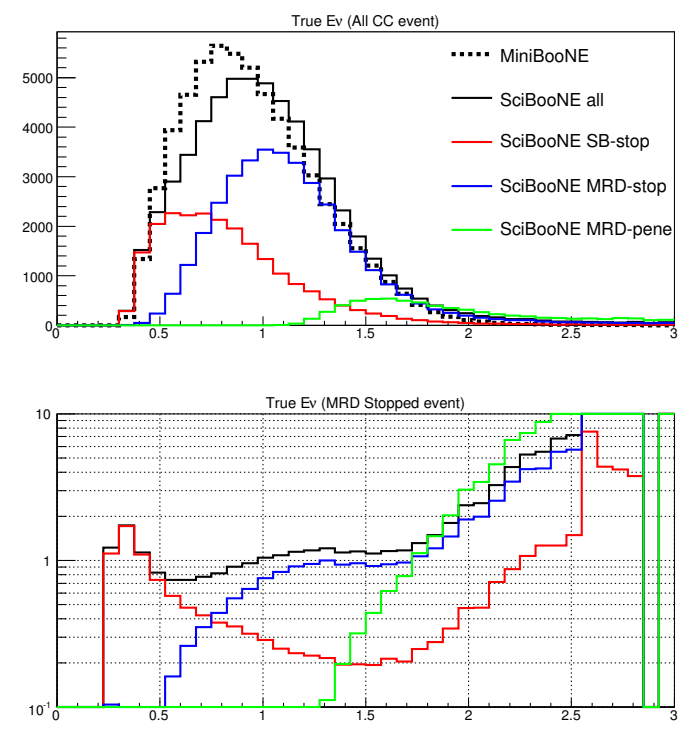

Figure A.4: Comparison of true $\theta_{\mu}$ distributions of selected sample at SciBooNE and MiniBooNE

Figure A.5: Comparison of true $E_{\nu}$ distributions of selected sample at SciBooNE and MiniBooNE 
Thus we adopt all these samples simultaneously (SciBar-stopped, MRD-stopped and MRDpenetrated samples), with the binning shown in the Table A.1

In this table, $f_{i}(i=0,1,2 \cdots)$ represents the scale factor for each $E_{\nu}$ region. We fit these parameters to minimize the $\chi^{2}$ between data and MC.

Table A.1: Scale factor for the spectrum fit

\begin{tabular}{lcccccc}
\hline \hline Parameter & $f_{0}$ & $f_{1}$ & $f_{2}$ & $f_{3}$ & $f_{4}$ & $f_{5}$ \\
\hline$E_{\nu}$ range $(\mathrm{GeV})$ & $0-0.5$ & $0.5-0.75$ & $0.75-1.0$ & $1.0-1.25$ & $1.25-1.75$ & $1.75-$ \\
\hline \hline
\end{tabular}

\section{A.2 Comparison of $\chi^{2}$ definitions}

We consider two definitions of the $\chi^{2}$ for the spectrum fitting: Log likelihood ratio and the Pearson's $\chi^{2}$. $\chi^{2}$.

In this section, we first describe about these two definitions, and then discuss the choice of the

\section{A.2.1 Likelihood ratio}

In the case of no systematic uncertainty present, the probability density function obeys Poisson distribution: $P(N, \mu)=\frac{\mu^{N} e^{-\mu}}{N !}$, where $N$ is the number of event observed, and $\mu$ is the expected number of event.

Using this function, the $\chi^{2}$ is written as:

$$
\chi^{2}=-2 \sum_{i}^{N \operatorname{Nbin}\left(P_{\mu}, \theta_{\mu}\right)} \ln \left[\frac{P\left(N_{i}^{\text {data }} ; N_{i}^{M C}\right)}{P\left(N_{i}^{\text {data }} ; N_{i}^{\text {data }}\right)}\right] .
$$

Then, we add systematic uncertainty. The systematic uncertainties are estimated bin-by-bin with their correlations. To incorporate this systematic error, we re-define the likelihood function as the convolution of Poisson and multivariate Gaussian distribution:

$$
P(\vec{n} ; \vec{\mu} ; V)=\frac{1}{(2 \pi)^{n / 2} \sqrt{|V|}} \int_{0}^{\infty} \prod_{i}^{N b i n} d x_{i} \frac{x_{i}^{n_{i}} e^{-x_{i}}}{n_{i} !} \exp \left[-\frac{1}{2}\left(x_{j}-\mu_{j}\right) V_{j k}^{-1}\left(x_{k}-\mu_{k}\right)\right]
$$

Here $V$ denotes the bin-by-bin error matrix with the dimension of Nbins $\times$ Nbins.

To calculate the above integration, we use Monte Carlo integration technique. Here, the likelihood function is calculated as:

$$
P(\vec{n} ; \vec{\mu} ; V)=\frac{1}{N \text { draws }} \sum_{i}^{\text {Ndraws }} P\left(\vec{n} ; \vec{x}_{i}\right), \quad \text { where } P\left(\vec{n} ; \vec{x}_{i}\right)=\prod_{j}^{N b i n} \frac{x_{i j}^{n_{j}} e^{-x_{i j}}}{n_{j} !} .
$$

In this equation, $\vec{x}_{i}$ is the $i$-th random draw of the expected number of event at each $P_{\mu}$ vs. $\theta_{\mu}$ bins, obtained by the error matrix $\mathrm{V}$ and the central value $\vec{\mu}$. We use 1000 random draws for this integration. The detail of the systematic uncertainties are described in the next section.

Using this likelihood function, we rewrite the definition of $\chi^{2}$ to:

$$
\chi^{2}=-2 \ln \left[\frac{P(\vec{n} ; \vec{\mu} ; V)}{P(\vec{n} ; \vec{n} ; V)}\right]
$$




\section{A.2.2 Pearson's $\chi^{2}$}

The other candidate is Pearson's $\chi^{2}$. In this case, the $\chi^{2}$ is defined as:

$$
\chi^{2}=\sum_{i, j}^{N b i n s}\left(n_{i}-\mu_{i}\right)\left(V_{\text {sys }}+V_{\text {stat }}\right)_{i j}^{-1}\left(n_{j}-\mu_{j}\right)
$$

where $V_{\text {sys }}$ is the same error matrix used for the likelihood method. $V_{\text {stat }}$ is defined as:

$$
V_{\text {stat }}=\left(\begin{array}{cccc}
n_{1} & & & 0 \\
& n_{2} & & \\
& & \ddots & \\
0 & & & n_{\text {Nbins }}
\end{array}\right)
$$

This method is less accurate compared to the likelihood method since we assume all systematic and statistical fluctuations are Gaussian around the predation $\mu$. However, the fit process is a lot simpler and stabler compare to the likelihood method using Monte Carlo integration.

\section{A.2.3 Comparison between the two methods}

We compare the two method by performing fits with statistical errors only. This way, we remove the uncertainties come from MC integration method and can see pure difference between Poisson and Gaussian assumptions.

Figure A.6 shows the fit results of the two methods. The parameters and their correlation coefficients for Poisson and Gaussian statistical errors are shown in the Tables A.2 and A.3, respectively. Here we can see the two fits give essentially same results, and it is safe to use Gaussian instead of Poisson distribution for the statistical error.

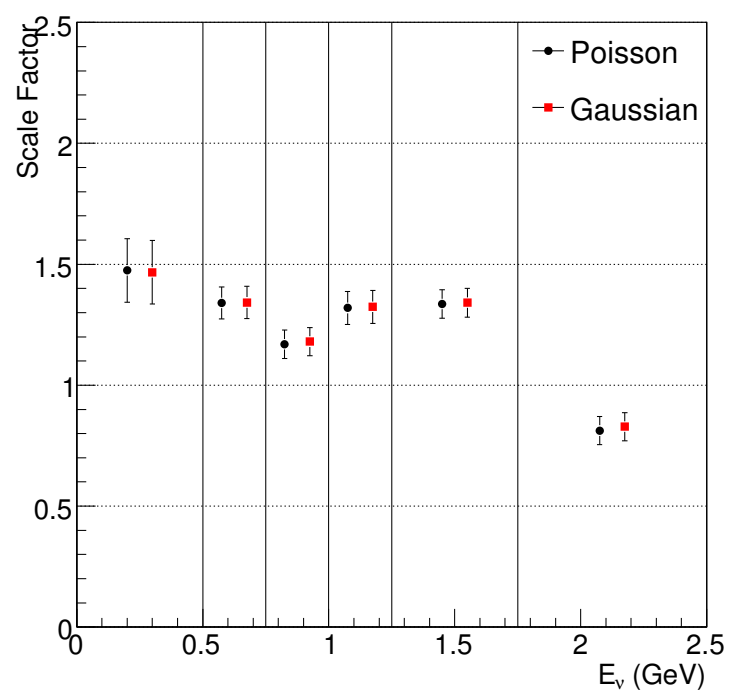

Figure A.6: Spectrum fit results with statistical errors only. The black points shows the fit results by Poisson log likelihood method described in the Section A.2.1. The red points shows the fit results by the Pearson's $\chi^{2}$ with Gaussian statistical error, described in the Section A.2.2.

For the Poisson Likelihood method, we need huge number of MC random draws to make an accurate expectation of the integral for systematic uncertainty. We found that the number of random draws are beyond what can be produced with the current computing power. Thus, we choose the error matrix method with Gaussian statistical error for the spectrum fitting. 
Table A.2: Best fit values and their error of the fit by the Poisson log likelihood method with statistic errors only. The binning used is the case B. The bottom half shows the correlation coefficients for each parameters.

\begin{tabular}{c|cccccc}
\hline \hline & $f_{0}$ & $f_{1}$ & $f_{2}$ & $f_{3}$ & $f_{4}$ & $f_{5}$ \\
\hline Best fit & 1.475 & 1.340 & 1.170 & 1.319 & 1.336 & 0.812 \\
Error & 0.132 & 0.066 & 0.059 & 0.069 & 0.059 & 0.058 \\
\hline$f_{0}$ & 1.0000 & -0.5155 & -0.0078 & 0.0420 & -0.0179 & -0.0185 \\
$f_{1}$ & -0.5155 & 1.0000 & -0.4737 & 0.0362 & -0.0042 & -0.0223 \\
$f_{2}$ & -0.0078 & -0.4737 & 1.0000 & -0.5818 & 0.1570 & -0.1089 \\
$f_{3}$ & 0.0420 & 0.0362 & -0.5818 & 1.0000 & -0.6144 & 0.2553 \\
$f_{4}$ & -0.0179 & -0.0042 & 0.1570 & -0.6144 & 1.0000 & -0.6130 \\
$f_{5}$ & -0.0185 & -0.0223 & -0.1089 & 0.2553 & -0.6130 & 1.0000 \\
\hline \hline
\end{tabular}

Table A.3: Best fit values and their error of the fit by the Pearson's $\chi^{2}$ with Gaussian statistical errors only. The binning used is the case B. The bottom half shows the correlation coefficients for each parameters.

\begin{tabular}{c|cccccc}
\hline \hline & $f_{0}$ & $f_{1}$ & $f_{2}$ & $f_{3}$ & $f_{4}$ & $f_{5}$ \\
\hline Best fit & 1.467 & 1.342 & 1.180 & 1.324 & 1.341 & 0.829 \\
Error & 0.132 & 0.067 & 0.059 & 0.068 & 0.060 & 0.058 \\
\hline$f_{0}$ & 1.0000 & -0.5302 & 0.0077 & 0.0396 & -0.0178 & -0.0172 \\
$f_{1}$ & -0.5302 & 1.0000 & -0.4846 & 0.0366 & 0.0017 & -0.0227 \\
$f_{2}$ & 0.0077 & -0.4846 & 1.0000 & -0.5706 & 0.1474 & -0.1072 \\
$f_{3}$ & 0.0396 & 0.0366 & -0.5706 & 1.0000 & -0.6201 & 0.2695 \\
$f_{4}$ & -0.0178 & 0.0017 & 0.1474 & -0.6201 & 1.0000 & -0.6265 \\
$f_{5}$ & -0.0172 & -0.0227 & -0.1072 & 0.2695 & -0.6265 & 1.0000 \\
\hline \hline
\end{tabular}




\section{Appendix B}

\section{CC single $\pi$ production $Q^{2}$ shape measurement}

\section{B.1 Sample to fit}

We found data-MC discrepancy at low-Q2 region for CC- $1 \pi$ enriched sample. To evaluate the size of the discrepancy, we fit the $Q^{2}$ distributions from SciBar-stopped and MRD-stopped samples.

First, each sample is subdivided into three sub-samples: 1 track, $\mu+p$ and $\mu+\pi$ samples. each sub-sample is defined as:

1 track: no additional tracks from the vertex.

$\mu+p: 1$ additional track from the vertex with MuCL $<0.05$.

$\mu+\pi: 1$ additional track from the vertex with MuCL $>0.05$.

Then, we reconstruct $Q^{2}$ assuming $\Delta(1232)$ mass $\left(Q_{\Delta}^{2}\right)$. Figure B.1 shows the resolution of $Q_{\Delta}^{2}$ for SciBar-stopped and MRD-stopped samples. We can see $Q_{\Delta}^{2}$ is a good estimate of true $Q^{2}$ value for CC-resonant $\pi$ interaction.
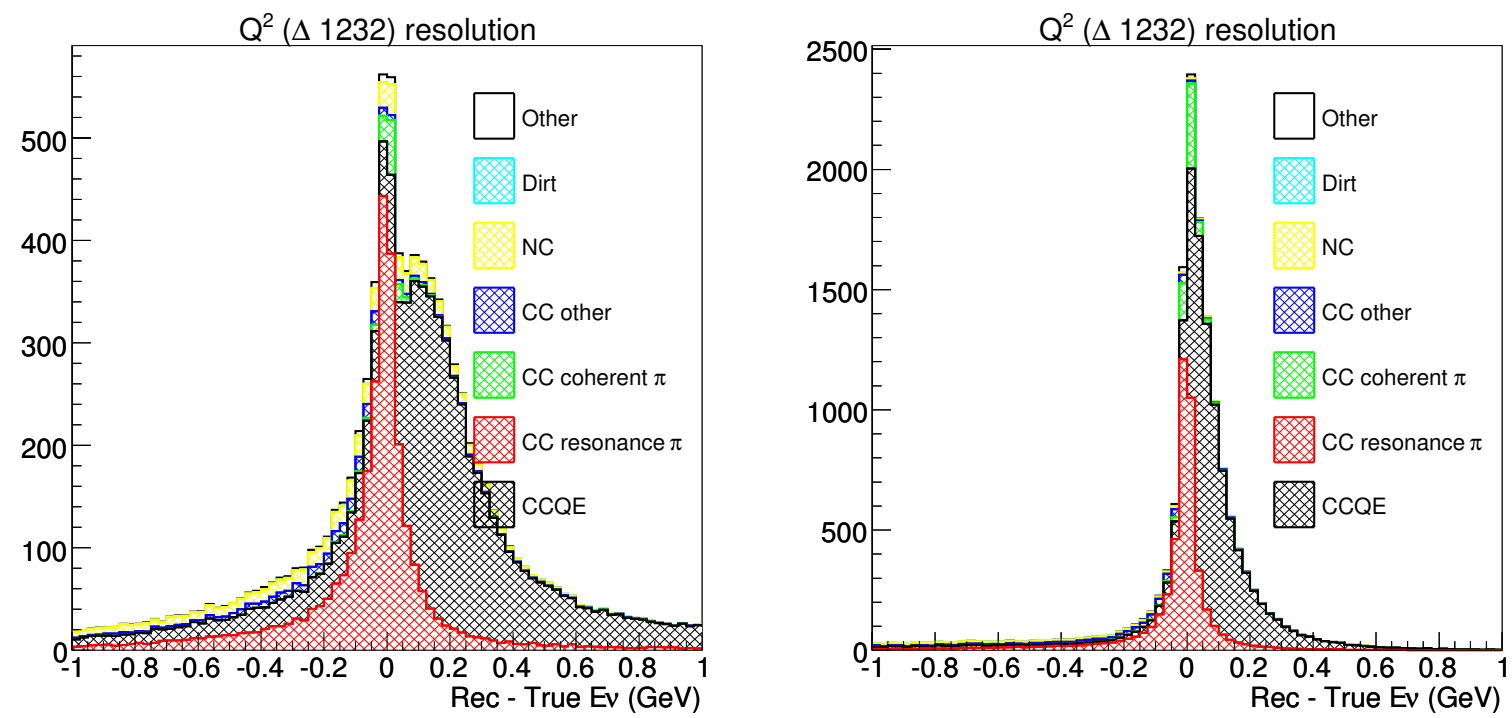

Figure B.1: Difference of $Q_{\Delta}^{2}$ and true $Q^{2}$ values from SciBar-stopped (left) and MRD-stopped (right) samples. 
Figures B.2 and B.3 show the distributions of $Q_{\Delta}^{2}$ for each sample before fitting. We fit these 6 distributions simultaneously to extract Q2 dependence of data-MC discrepancy for $\mathrm{CC}-1 \pi$ production.
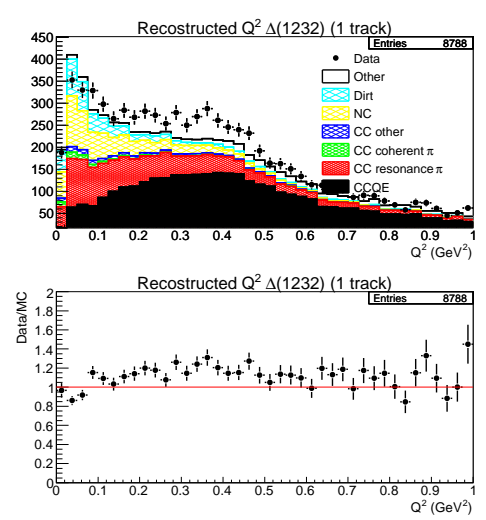
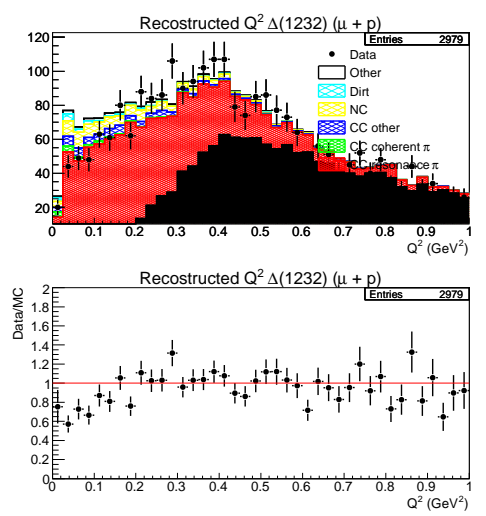
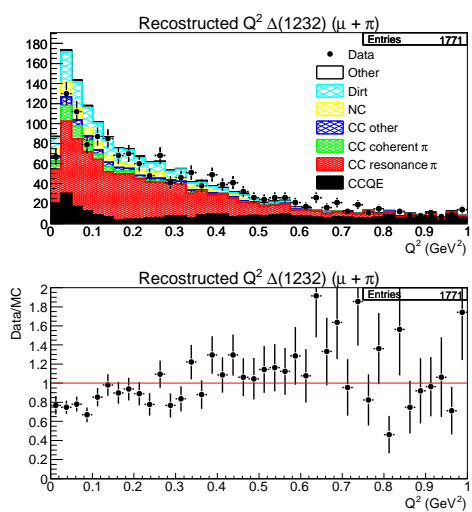

Figure B.2: Reconstructed $Q_{\Delta}^{2}$ distributions for 1 track (left), $\mu+p$ (center), and $\mu+\pi$ (right) samples from SciBar-stopped events.
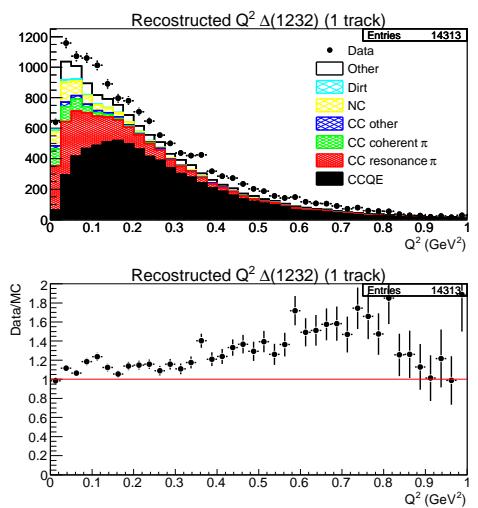
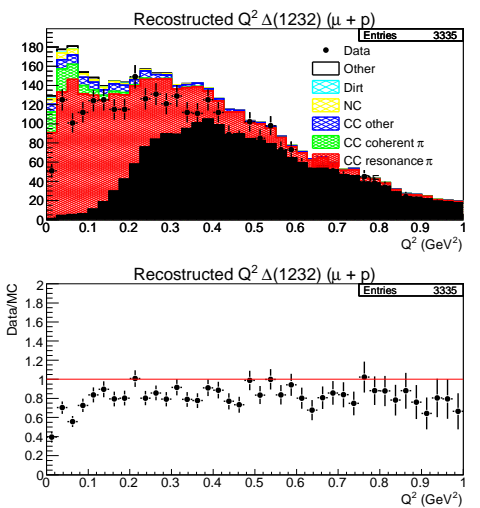
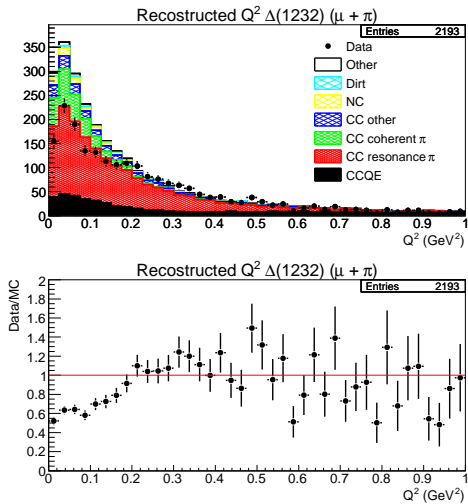

Figure B.3: Reconstructed $Q_{\Delta}^{2}$ distributions for 1 track (left), $\mu+p$ (center), and $\mu+\pi$ (right) samples from MRD-stopped events.

\section{B.2 Fit method and result}

Since the significant data-MC discrepancy is present at $Q_{\Delta}^{2}<0.2 \mathrm{GeV}$, we assume the correction function to be:

$$
P_{1 \pi}=\left\{\begin{array}{ll}
A_{1 \pi} \times Q_{(\Delta)}^{2}+B_{1 \pi} & \left(Q_{(\Delta)}^{2} \leq 0.2 \mathrm{GeV}\right) \\
1 & \left(Q_{(\Delta)}^{2}>0.2 \mathrm{GeV}\right)
\end{array} .\right.
$$

Including these correction factors $\left(A_{1 \pi}, B_{1 \pi}\right)$, the following 6 parameters are used for the fitting.:

$A_{1 \pi}, B_{1 \pi}:$ Correction factor for CC resonant $1 \pi$ production.a

$P_{C o h-\pi}:$ Scale factor for CC coherent $\pi$ production.

$P_{\text {norm }}$ : Overall normalization factor.

$P_{Q E / n o n-Q E}:$ Migration factor between $\mathrm{QE}$ and non-QE events.

$P_{1 \text { trk } / 2 \text { trk }}:$ Migration factor between 1 track and 2 track samples. 
We fit these parameters with the statistical errors only. The $\chi^{2}$ is the ratio of the Poisson log likelihood defined as

$$
\chi^{2}=-2 \sum_{i}^{N b i n s} \ln \left[\frac{P\left(N_{i}^{\text {data }} ; N_{i}^{M C}\right)}{P\left(N_{i}^{\text {data }} ; N_{i}^{\text {data }}\right)}\right], \quad \text { where } \quad \mathrm{P}(\mathrm{N}, \mu)=\frac{\mu^{\mathrm{N}} \mathrm{e}^{-\mu}}{\mathrm{N} !}
$$

Table B.1 shows best fit value of the parameters.

Table B.1: Best fit values of the CC- $1 \pi \mathrm{Q}^{2}$ fitting.

\begin{tabular}{|c|c|c|c|c|c|c|}
\hline Parameter & $A_{1 \pi}$ & $B_{1 \pi}$ & $P_{C o h-\pi}$ & $P_{\text {norm }}$ & $P_{Q E / n o n-Q E}$ & $P_{1 t r k / 2 t r k}$ \\
\hline Best fit & 2.14 & 0.43 & 0.56 & 1.24 & 0.88 & 1.31 \\
\hline
\end{tabular}

Although these factors are obtained as a function of $Q_{(\Delta)}^{2}$, we apply these factor as a function of true $Q^{2}$. Figures B.4 and B.5 are the $Q_{(\Delta)}^{2}$ distribution after applying these scale factors. We can see the obtained scale factor as a function of true $Q^{2}$ still well describing the data-MC discrepancy at low $Q^{2}$.
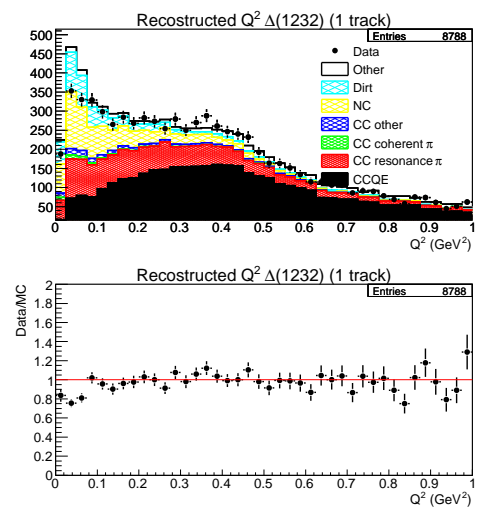
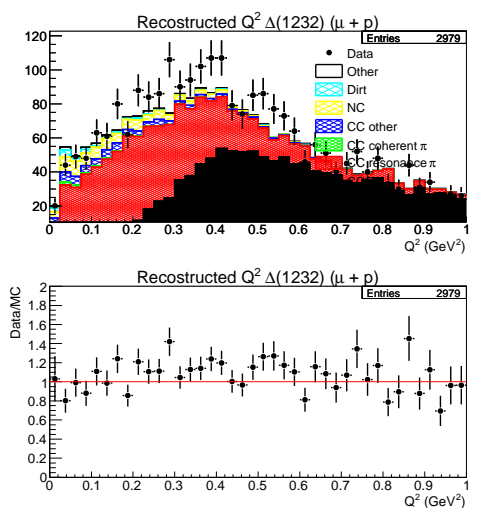
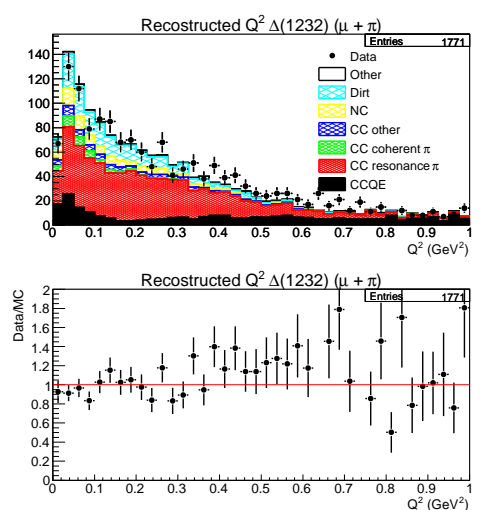

Figure B.4: Reconstructed $Q_{\Delta}^{2}$ distributions for 1 track (left), $\mu+p$ (center), and $\mu+\pi$ (right) samples from SciBar-stopped events, after the fitting.
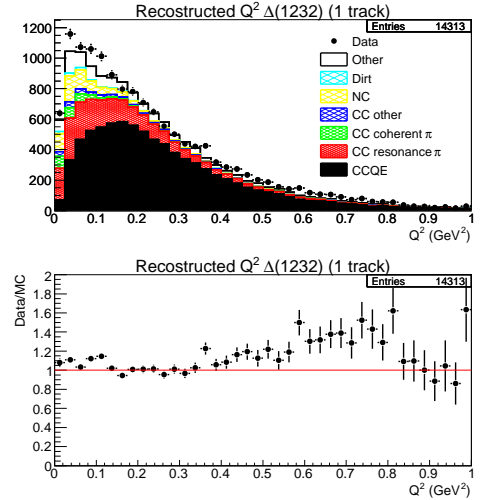
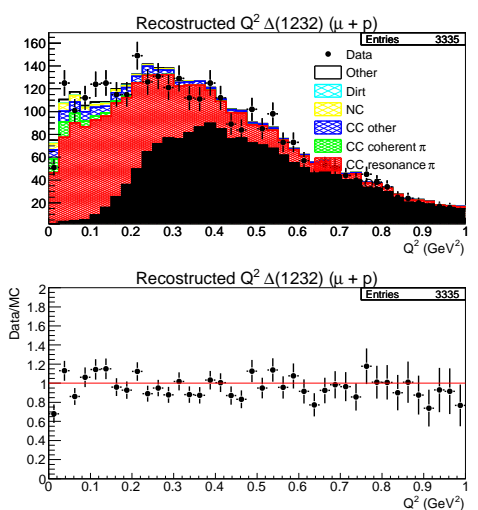
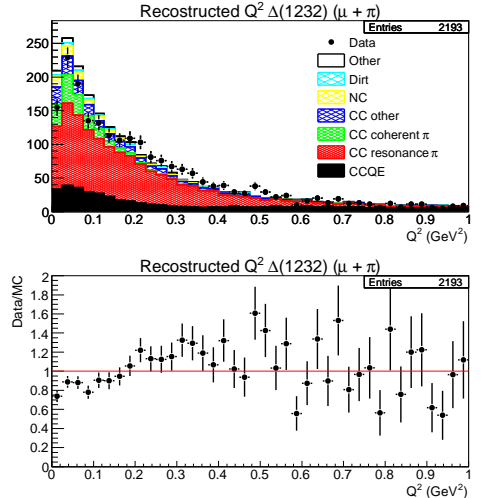

Figure B.5: Reconstructed $Q_{\Delta}^{2}$ distributions for 1 track (left), $\mu+p$ (center), and $\mu+\pi$ (right) samples from MRD-stopped events, after the fitting.

Then, another assumption is that the effect of $P_{n o r m}, P_{Q E / n o n-Q E}$ and $P_{1 \text { trk/2trk }}$ are already covered by the current systematic error for flux, $M_{A}$ and FSI errors. Hence, only the effect from $P_{C o h-\pi}, A_{1 \pi}$ and $B_{1 \pi}$ are taken into account for the systematic uncertainty for the spectrum fitting (and near to far extrapolation). 


\section{B.3 Comparison to the correction factor obtained from MiniBooNE data}

Finally we check the consistency of the CC- $1 \pi$ correction factor with the one obtained from MiniBooNE data.

Figure B.6 is the reconstructed $Q_{Q E}^{2}$ of MiniBooNE CC- $1 \pi$ event. Both SciBooNE- and MiniBooNE-based corrections are overlaid in the plot. We confirm that the behaviour at low$Q^{2}\left(Q^{2}<0.2 \mathrm{GeV}\right)$ is very similar. Although there is a little discrepancy between two corrections, that effect is presumably covered by the $M_{A}$ and FSI uncertainties.

Then, we use SciBooNE-based correction on both MiniBooNE and SciBooNE for the oscillation analysis.
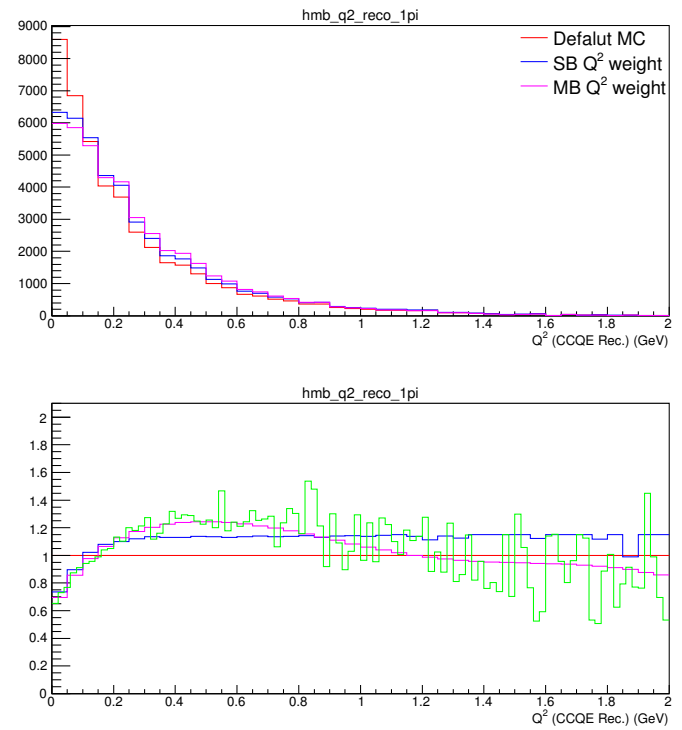

Figure B.6: Comparison of the correction based on SciBooNE and MiniBooNE. The top plot is the $Q_{Q E}^{2}$ distribution of (MC true) CC$1 \pi$ events. Distributions of default prediction, the one after SciBooNE based correction and the one after MiniBooNE based correction are overlaid. The bottom plot is the ratio to the default prediction. 


\section{Appendix C}

\section{Simulation and event reconstruction in MiniBooNE}

\section{C.1 MiniBooNE detector simulation}

Propagation of final state particles emerging from the target nucleus in the MiniBooNE detector are simulated by a GEANT3 [111] framework. In this simulation, photons are generated through Cherenkov radiation and scintillation, and propagated until they hit PMTs. The responses of PMT and DAQ electronics are simulated separately.

For simulating of hadronic interactions, we use GCALOR [112] package, which reproduce pion absorptions and charge exchange processes well, instead of GFLUKA [113] model which implemented as the default package in GENAT3. The differences of two codes are used to estimate the systematic uncertainties.

Propagation of photons through the oil are simulated using a custom model tuned with a combination of external measurements and calibration data. Figure C.1 shows the rates of optical photon processes as a function of wavelength. The reflections of photons at the tank walls in both the main tank and veto regions are also implemented into the simulation.

A detailed description of MiniBooNE detector simulation is available in Ref. [108].

\section{C.1.1 MiniBooNE detector response error}

For the MiniBooNE detector response error, following sources are taken into account:

- PMT efficiency

- Reflections in the tank

- Oil optical properties

- Electronics model

- Nuclear effects in the detector

Among these, the largest contribution to the detector error is from the optical properties of the oil, which includes uncertainties of attenuation length, Rayleigh/Raman scattering, refractive index, scintillation yields, fluorescence yields. We introduce 39 parameters to describe these effects, and estimate the uncertainties of these parameters. Then, the errors of these parameters are propagated to the MiniBooNE reconstructed $E_{\nu}$ distribution.

As for the nuclear effects in the detector, we include uncertainties of $\pi$ absorption and charge exchange cross sections. The size of uncertainties are set to $35 \%$ and $50 \%$ for $\pi$ absorption and charge exchange cross sections, respectively. 


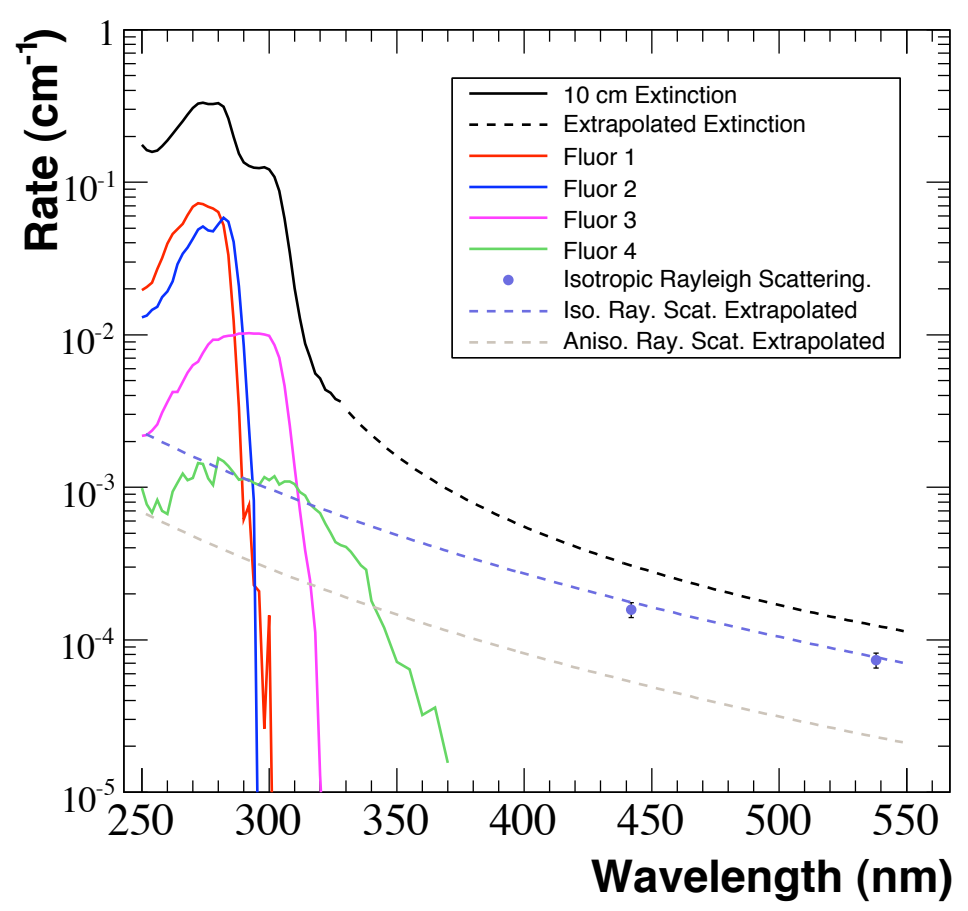

Figure C.1: Rates of optical photon processes in the Marcol 7 oil used in MiniBooNE. The total extinction was measured in a $10 \mathrm{~cm}$ cell at FNAL. The extrapolated extinction curve is obtained using the calibration data by laser flask and Michel electrons. The rates of fluorescence and Rayleigh scattering are also shown. This plot is taken from Ref. [108]

We form a error matrix for the MiniBooNE detector response uncertainty ( $\mathbf{V}^{\text {det }}$ ) by adding these sources together. More detailed descriptions about the MiniBooNE detector response errors can be found elsewhere [82, 108].

\section{C.2 Selection of beam-induced events}

To select neutrino interaction in MiniBooNE detector, we first define "subevents" defined as clusters of PMT hits separated in time. Specifically, more than 10 PMT hits need to occur with no more than $10 \mathrm{~ns}$ between consecutive hits to be categorized as a subevent.

Figure C.2 shows the time window for all events in MiniBooNE. The beam time window is centered on $\sim 5000 \mathrm{~ns}$ and we can clearly see the beam-induced events clustered there. Outside the beam window, we can see a flat rate due to cosmic ray induced events, with additional exponential decay structure within the beam window and trailing the beam window which caused by electrons from muon decay. To remove the decay electron events, we require the reconstructed a minimal energy is required, as electrons from muon decay all have energy less than $52.3 \mathrm{MeV}$. By requiring enough tank hits (> 200 main tubes fired) and the exponential structure (and the electrons) vanish. If additionally the events are required to be contained with minimal activity in the veto $(<6$ veto tubes fired), then rightmost plot in Fig. C.2 shows a flat distribution of neutrino candidate events in only the beam window.

These "precuts" (main tank hits $<200$ and veto hits $<6$ ) select only neutrino induced interactions caused by the beam, and are applied for all further discussions of selection. 

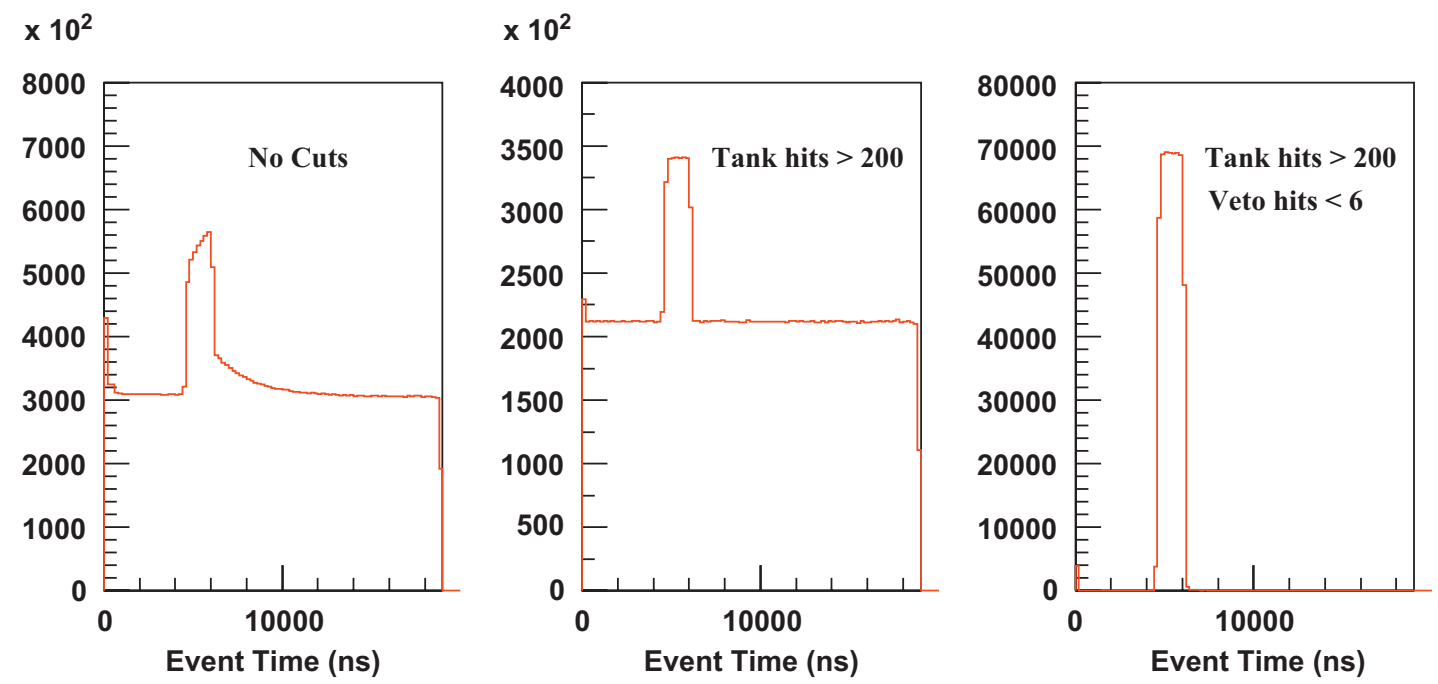

Figure C.2: Average time (ns) of the first subevent for all events in MiniBooNE. Left: No selection is applied. Middle: A minimal energy threshold is required (main tank hits $>200$ ). Right: Containment is also required with minimal activity in the veto (veto hits $<6$ ). Plots taken from Ref. [80].

\section{C.3 Track reconstruction}

An example of a muon event is shown in Figure C.3.

A single particle track can be characterized by the following parameters:

- position $(x, y, z)$

- direction $\left(u_{x}, u_{y}, u_{z}\right)$

- kinetic energy $(E)$

- time $(T)$

We reconstruct these parameters using the charge $\left(q_{i}\right)$ and timing $\left(t_{i}\right)$ information obtained from the all 1280 PMTs, where $i$ is an index of PMT. Using these quantities, we form a likelihood function for the parameter set, $\vec{\alpha}=\left(x, y, z, u_{x}, u_{y}, u_{z}, E, T\right)$, as:

$$
L(\vec{\alpha})=\prod_{i=1}^{1280} P\left(q_{i} \mid \vec{\alpha}\right) P\left(t_{i} \mid \vec{\alpha}\right),
$$

where $P\left(q_{i} \mid \vec{\alpha}\right)$ is the probability of measuring a charge $q_{i}$ at PMT $i$ for a track with parameters $\vec{\alpha}$, and $P\left(t_{i} \mid \vec{\alpha}\right)$ is the same for the measured time $t_{i}$. The parameter set, $\vec{\alpha}$, is determined by minimizing the negative log likelihood function defined as:

$$
-\log (L(\vec{\alpha}))=-\sum_{i=1}^{1280} \log \left(P\left(q_{i} \mid \vec{\alpha}\right)\right)-\sum_{i=1}^{1280} \log \left(P\left(t_{i} \mid \vec{\alpha}\right)\right) .
$$

Figure C.4 compares true muon variables to reconstructed ones in a series of profile histograms. In this fit, we assume that Cherenkov lights are emitted from a point source, although, in the reality, the emission points are distributed along the tracks. Even though, we can see that this reconstruction method models muons acceptably and behaves similarly for data and prediction. This method has since been improved [114, but is not used for the work in this thesis. A more detail about the likelihood function and the fit method can be found elsewhere [114, 115]. 


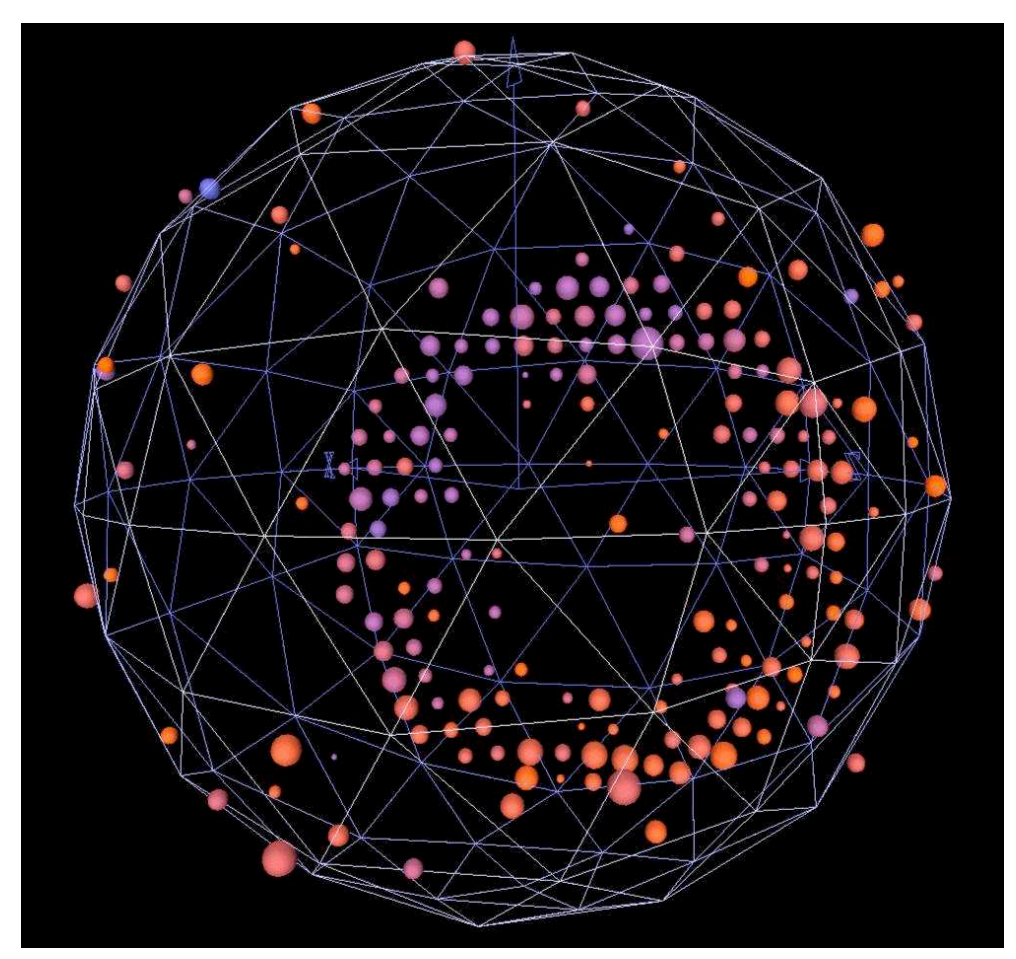

Figure C.3: A typical muon event in MiniBooNE. The white frame represents the inner tank surface. Color indicates time (red $\rightarrow$ blue is early to late time), and size of the spheres represents the amount of charge deposited.

\section{C.4 Event selection in MiniBooNE}

In this analysis, we select CCQE-enriched sample. A signal of muon track contained in the tank (the first subevent) is usually followed by another subevent due to its decay electron (the second subevent) as shown in Fig. C.5. Figure C.6 shows the timing distribution of a typical CCQE candidate event. The CCQE event sample is selected by identifying these two subevents, with the same criteria as were used for the measurement of quasi-elastic scattering on carbon [48] and the MiniBooNE-only $\nu_{\mu}$ disappearance analysis [44]. We require exactly two subevents are required (the muon and the decay electron). To reject cosmic ray interactions, both subevents are required to have less than 6 veto-PMT hits. The first subevent must be in coincidence with a beam pulse, have a reconstructed track center less than $500 \mathrm{~cm}$, and greater than 200 in tank PMT hits to eliminate electrons from cosmic ray muon decays. The second subevent must have less than 200 inner PMT hits to be below the decay electron energy endpoint.

A CC $\pi^{+}$event will produce three subevents:the muon, the muon's decay electron, and the pion's decay muon's decay electron. Hence, most of $\mathrm{CC} \pi^{+}$events are rejected with this selection.

Then, we also require the distance between the electron vertex and the muon track endpoint must be less than $100 \mathrm{~cm}$, to ensure that the electron decayed from the muon. The muon track endpoint is calculated assuming the first subevent is a MIP, and using the energy and direction to calculate the endpoint. This cut preferentially eliminates $\mathrm{CC} \pi^{+}$events, as the decay electron from the pion can be far from the muon's endpoint.

The selection cuts are $35 \%$ efficient within the fiducial volume of $500 \mathrm{~cm}$, which is the product of:

- $50 \%$ probability to contain (muon) events within the inner tank.

- $83 \%$ probability of tagging a muon with a decay electron ( $7 \%$ of $\mu^{-}$capture on carbon[118])

- $85 \%$ probability that the electron of the second subevent is close to the endpoint of the 

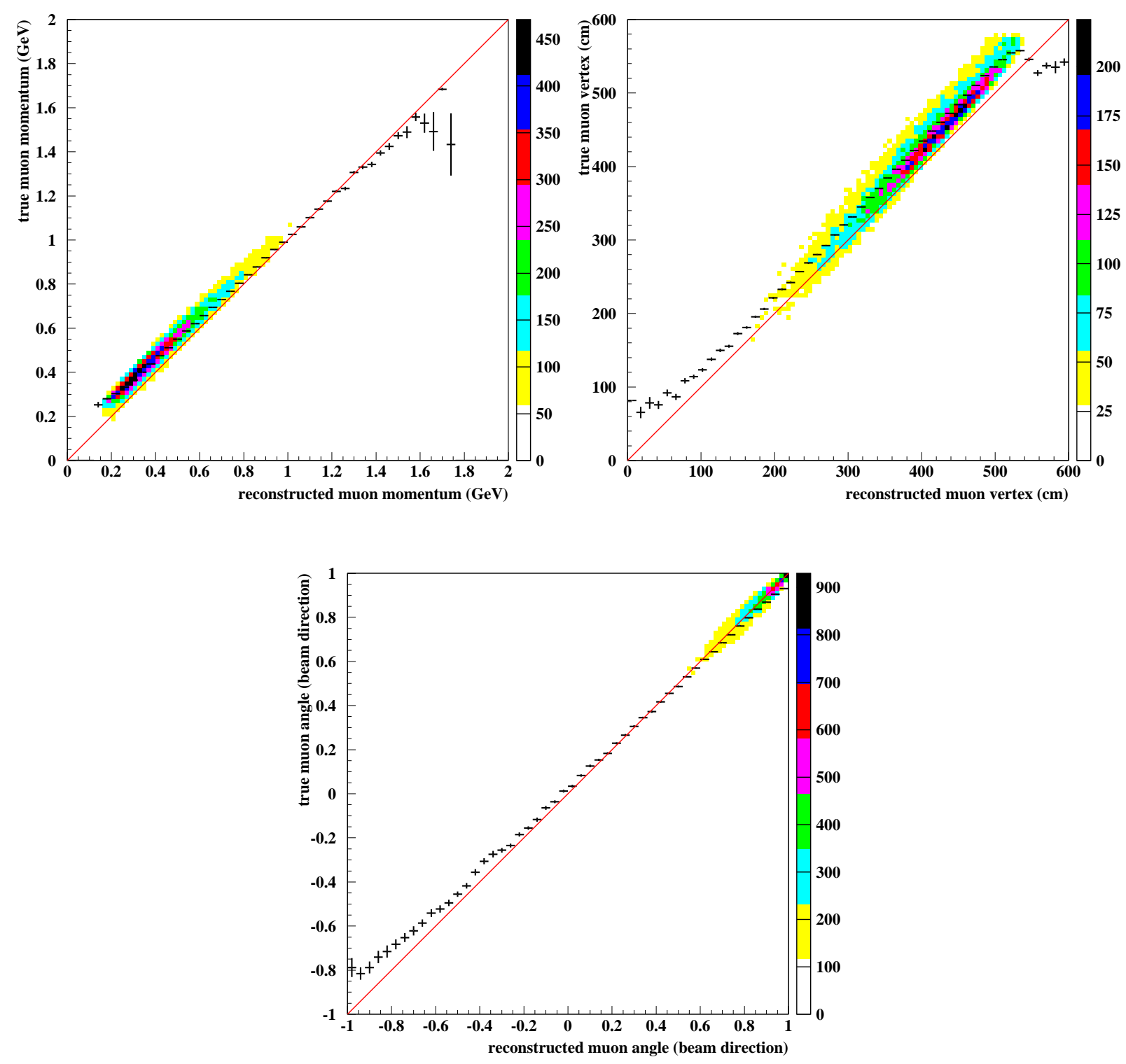

Figure C.4: Reconstructed versus true muon variables. The black crosses indicate the spread of the distribution (profile histogram) while the color scale is a 2D plot. Top left: Muon momentum $(\mathrm{GeV})$. Top right: Distance from center of the tank to the vertex (radius). Bottom: $\cos \left(\theta_{\text {beam }}\right)$, the angle of the muon with respect to the beam direction. 


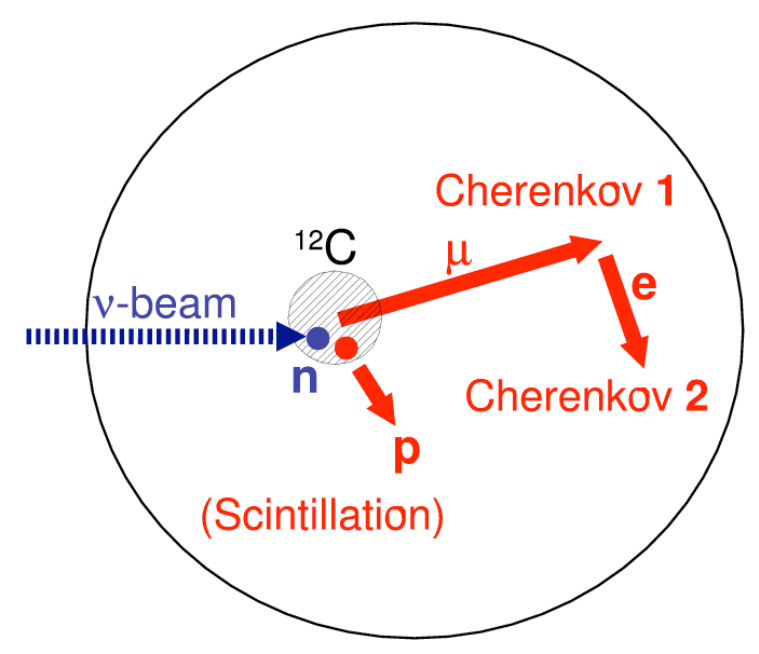

Figure C.5: Schematics of a CCQE interaction occurred in the MiniBooNE detector. The primary Cherenkov light from the muon (Cherenkov 1, first subevent) and subsequent Cherenkov light from the decayed electron (Cherenkov 2, second subevent) are used to select CCQE interactions. The scintillation light emitted by protons are isotropic and not sensitive for this analysis. Plot taken from Ref. [116].

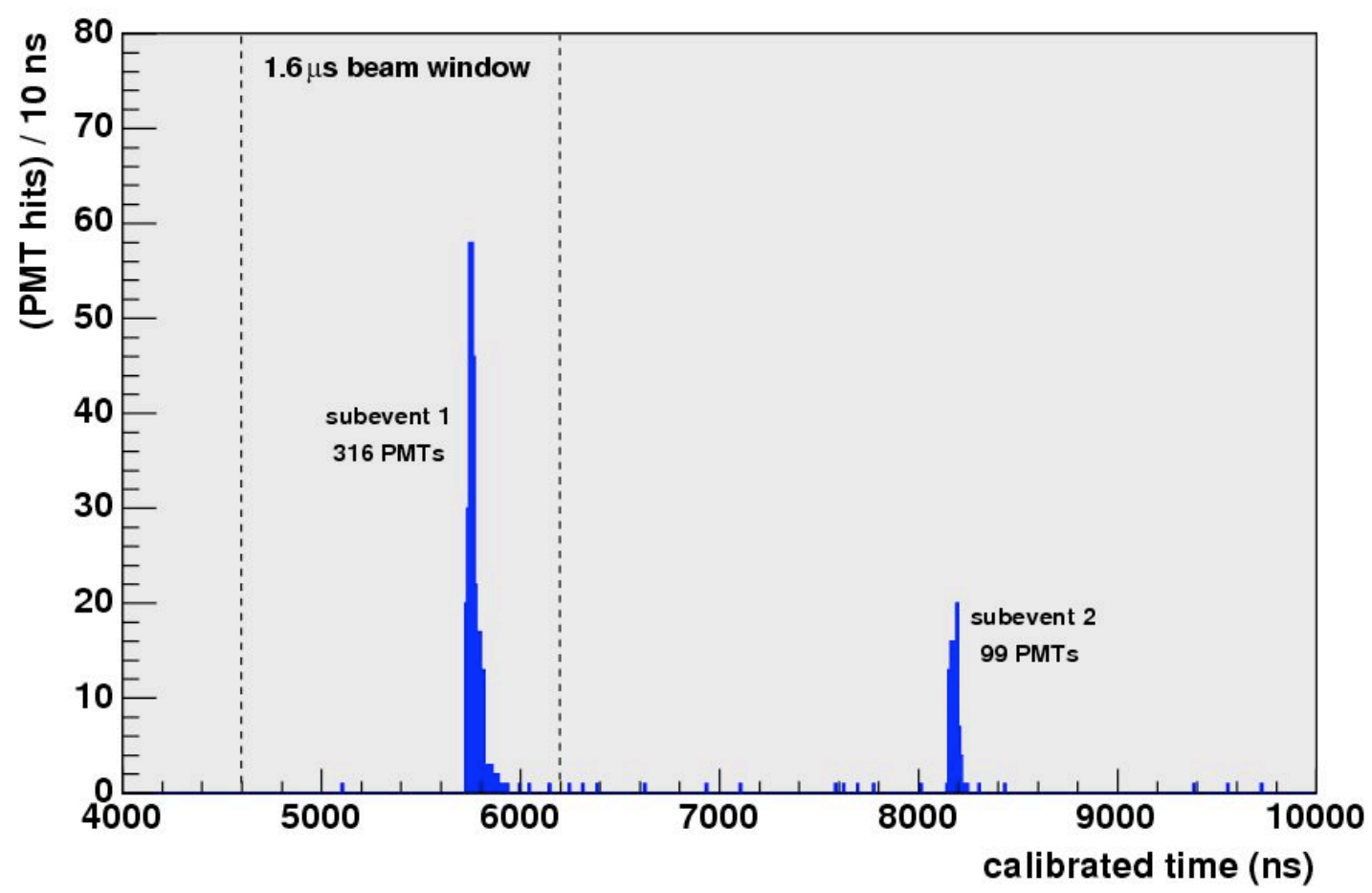

Figure C.6: Timing distribution of the PMT hits for a single beam trigger with two subevents. This events is a $\nu_{\mu}$ CCQE event candidate where the first subevent is the signal of the muon and the second is its decay electron. Plot taken from Ref. [117] 
muon (first subevent)

According to the MC simulation, the $\nu_{\mu}$ sample is $74 \% \mathrm{CCQE}, 21 \% \mathrm{CC} \pi^{+}$and $4 \% \mathrm{CC} \pi^{0}$. The remaining $1 \%$ are multi-pion events or neutral current pion production events. For a $\mathrm{CC} \pi$ to become background, the outgoing pion must be unobserved, and thus produce no third subevent. This can happen when the pion is absorbed in the nucleus, decays along the muon track, or when the pion's decay electron does not produce enough hits to create a third subevent.

\section{C.5 Neutrino energy reconstruction}

We reconstruct neutrino energy from muon kinematics assuming the CCQE interaction using Eq. (5.2). Then, an additional set of corrections is applied in both data and prediction samples to create a better estimate of the neutrino energy [115].

1. A correction as a function of the muon energy.

2. A correction to reconstructed neutrino energy as a function of $Q^{2}$.

3. A final correction as a function of the reconstructed neutrino energy.

Figure C.7 shows the corrected $E_{\nu}^{Q E}$ distribution as compared to $E_{\nu}$ for events in the MiniBooNE sample. Like SciBooNE, the CCQE events reconstruct $E_{\nu}^{Q E}$ close to $E_{\nu}$, but $\mathrm{CC} \pi^{+}$background events reconstruct at the wrong value of $E_{\nu}^{Q E}$ due to the assumption that they are quasi-elastic.
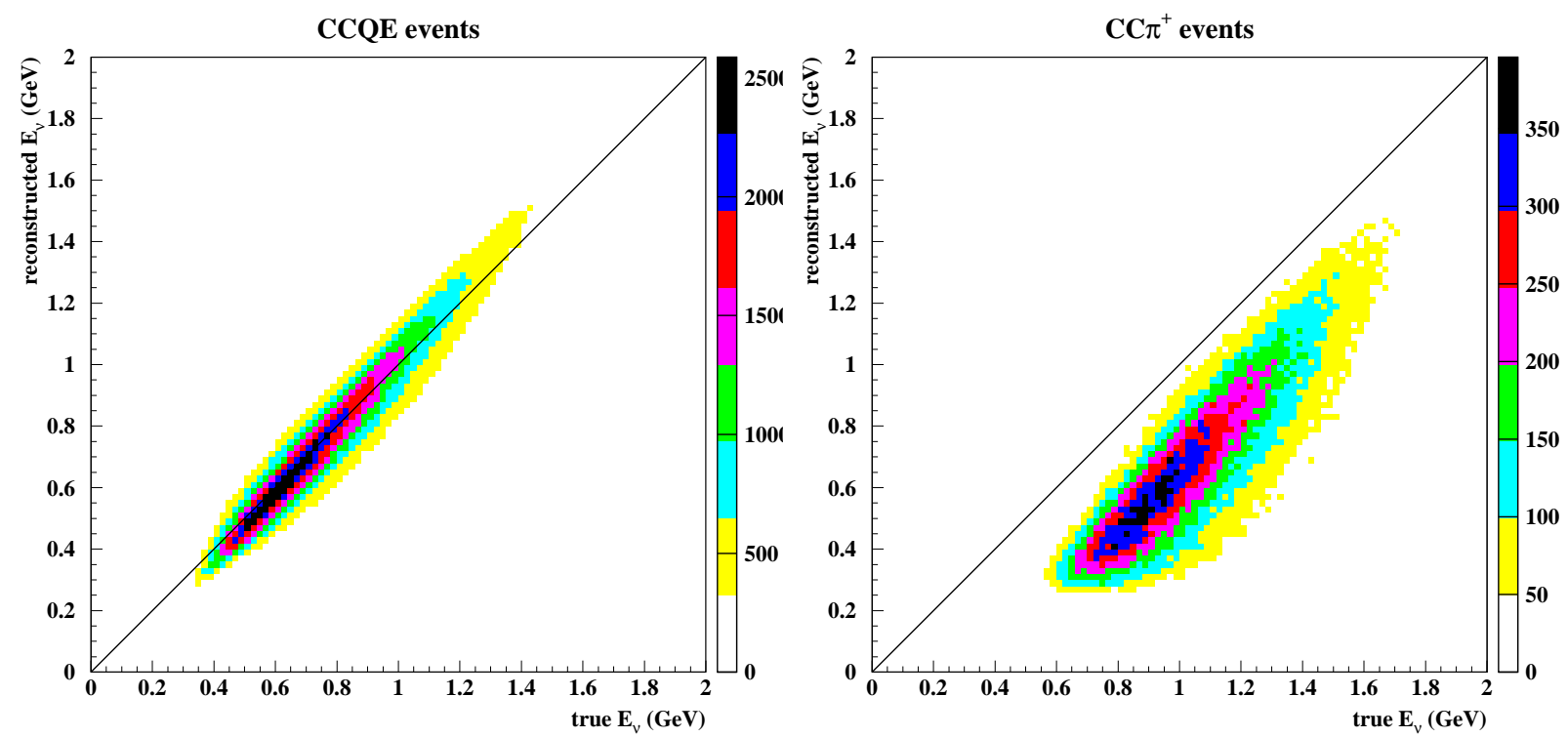

Figure C.7: $2 \mathrm{D}$ histogram of reconstructed $E_{\nu}^{Q E}$ vs. generated $E_{\nu}$ for CCQE (left) and CC $\pi^{+}$ (right) events in MiniBooNE; the black line is at $\mathrm{y}=\mathrm{x}$.

Figure C.8 show the distribution of reconstructed $E_{\nu}$ at MiniBooNE. The contamination of backgrounds CC non-QE interaction are also shown in this figure. The energy resolution is shown in Fig. C.9 as a function of true neutrino energy for CCQE events in the MiniBooNE sample. 


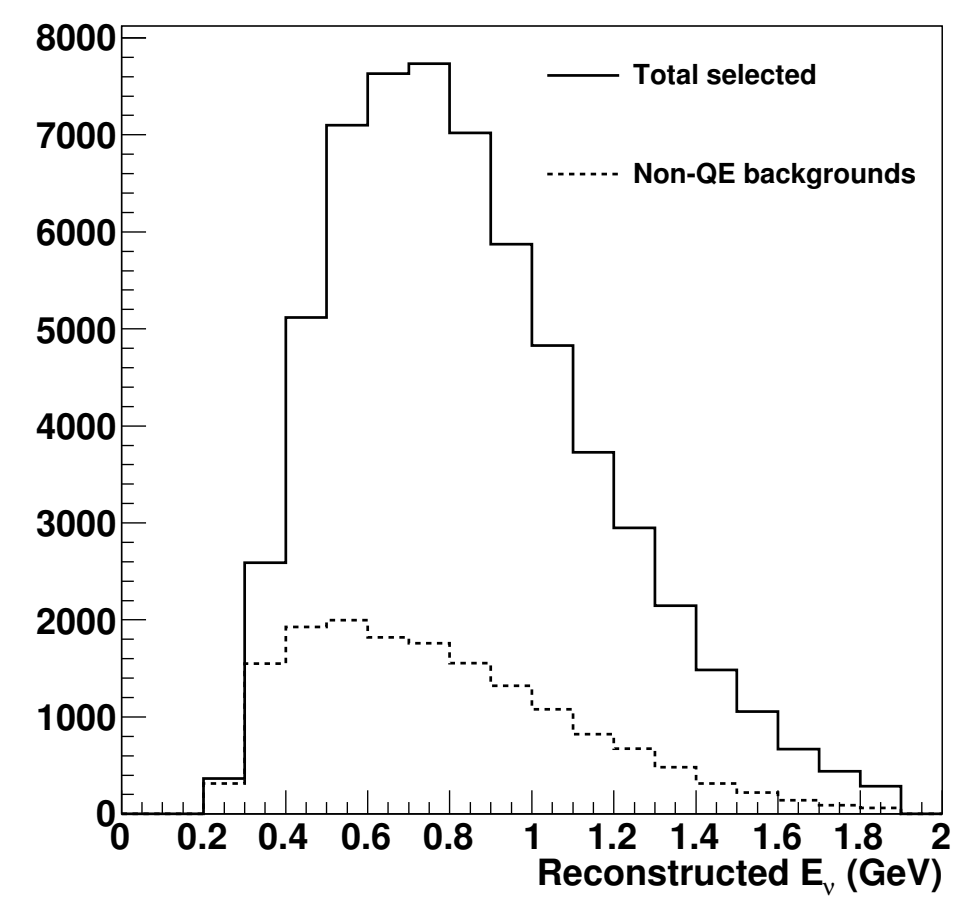

Figure C.8: Distribution of reconstructed $E_{\nu}$ at MiniBooNE. The contamination of backgrounds $\mathrm{CC}$ non-QE interaction are also shown.

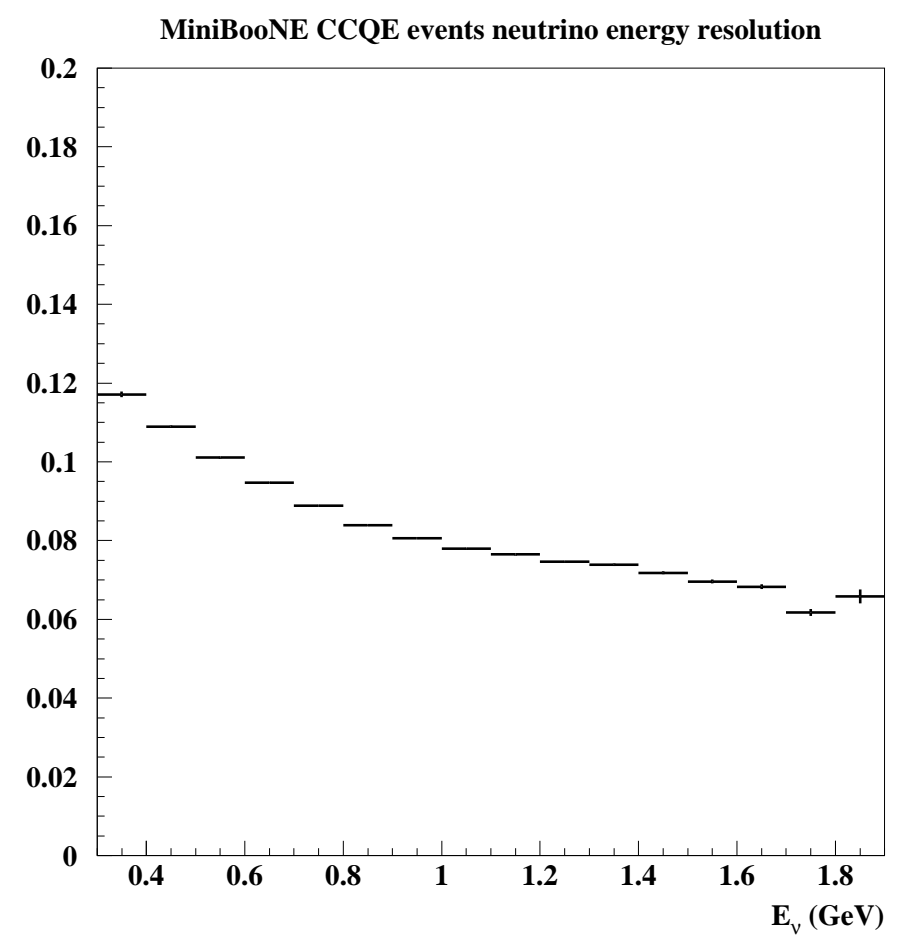

Figure C.9: Neutrino energy resolution (reconstructed $E_{\nu}^{Q E}$-generated $E_{\nu}$ )/ generated $E_{\nu}$, for CCQE events in the MiniBooNE CCQE sample, as a function of generated $E_{\nu}$ in $100 \mathrm{MeV}$ bins. 


\section{Bibliography}

[1] Phys. Rept. 427, 257 (2006), arXiv:hep-ex/0509008.

[2] C. Kraus et al., Eur. Phys. J. C40, 447 (2005), arXiv:hep-ex/0412056.

[3] K. Assamagan et al., Phys. Rev. D 53, 6065 (1996).

[4] R. Barate et al. (ALEPH Collaboration), Eur. Phys. J. C2, 395 (1998),

[5] B. Kayser, arXiv:hep-ph/0211134.

[6] M. Fukugita and T. Yanagida, Physics of neutrinos and applications to astrophysics (Springer, 2003).

[7] Z. Maki, M. Nakagawa, and S. Sakata, Prog. Theor. Phys. 28, 870 (1962),

[8] M. Gell-Mann, P. Ramond, and R. Slansky, Supergravity (North Holland, Amsterdam, 1979).

[9] T. Yanagida, in Proceedings of the Workshop on Unified Theory and Baryon Number in the Universe, edited by O. Sawada and A. Sugamoto (KEK, Tsukuba, Japan, 1979).

[10] R. N. Mohapatra and G. Senjanovic, Phys. Rev. Lett. 44, 912 (1980).

[11] R. N. Mohapatra and G. Senjanovic, Phys. Rev. D 23, 165 (1981).

[12] Y. Ashie et al. (Super-Kamiokande Collaboration), Phys. Rev. D 71, 112005 (2005), arXiv:hep-ex/0501064.

[13] M. H. Ahn et al. (K2K Collaboration), Phys. Rev. D 74, 072003 (2006), arXiv:hep-ex/0606032

[14] D. G. Michael et al. (MINOS Collaboration), Phys. Rev. Lett. 97, 191801 (2006), arXiv:hepex/0607088.

[15] B. T. Cleveland et al., Astrophys. J. 496, 505 (1998).

[16] W. Hampel et al. (GALLEX Collaboration), Phys. Lett. B447, 127 (1999).

[17] M. Altmann et al. (GNO Collaboration), Phys. Lett. B616, 174 (2005), arXiv:hep-ex/0504037

[18] J. N. Abdurashitov et al. (SAGE Collaboration), Phys. Rev. C 80, 015807 (2009), arXiv:0901.2200 [nucl-ex] .

[19] B. Aharmim et al. (SNO Collaboration), Phys. Rev. C 72, 055502 (2005), arXiv:nuclex/0502021.

[20] J. Hosaka et al. (Super-Kamkiokande Collaboration), Phys. Rev. D 73, 112001 (2006), arXiv:hep-ex/0508053. 
[21] C. Arpesella et al. (Borexino Collaboration), Phys. Rev. Lett. 101, 091302 (2008), arXiv:0805.3843 [astro-ph].

[22] S. Abe et al. (KamLAND Collaboration), Phys. Rev. Lett. 100, 221803 (2008), arXiv:0801.4589 [hep-ex] .

[23] A. Aguilar et al. (LSND Collaboration), Phys. Rev. D 64, 112007 (2001), arXiv:hepex/0104049.

[24] A. A. Aguilar-Arevalo et al. (MiniBooNE Collaboration), Phys. Rev. Lett. 98, 231801 (2007), arXiv:0704.1500 [hep-ex].

[25] A. A. Aguilar-Arevalo et al. (MiniBooNE Collaboration), Phys. Rev. Lett. 102, 101802 (2009), arXiv:0812.2243 [hep-ex] .

[26] A. A. Aguilar-Arevalo et al. (MiniBooNE Collaboration), Phys. Rev. Lett. 105, 181801 (2010), arXiv:1007.1150 [hep-ex].

[27] M. Sorel, J. M. Conrad, and M. Shaevitz, Phys. Rev. D 70, 073004 (2004), arXiv:hep$\mathrm{ph} / 0305255$.

[28] G. Karagiorgi et al., Phys. Rev. D 75, 013011 (2007), arXiv:hep-ph/0609177.

[29] G. Karagiorgi, Z. Djurcic, J. M. Conrad, M. H. Shaevitz, and M. Sorel, Phys. Rev. D 80, 073001 (2009), arXiv:0906.1997 [hep-ph] .

[30] M. Maltoni, J. Phys. Conf. Ser. 110, 082011 (2008), arXiv:0711.2018 [hep-ph] .

[31] M. Maltoni and T. Schwetz, Phys. Rev. D 76, 093005 (2007), arXiv:0705.0107 [hep-ph].

[32] G. S. Karagiorgi, Ph.D. thesis, Massachusetts Institute of Technology (2010), FERMILABTHESIS-2010-39.

[33] Y. Declais et al., Nucl. Phys. B434, 503 (1995).

[34] M. Apollonio et al. (CHOOZ Collaboration), Eur. Phys. J. C27, 331 (2003), arXiv:hepex/0301017.

[35] I. E. Stockdale et al., Phys. Rev. Lett. 52, 1384 (1984).

[36] F. Dydak et al., Phys. Lett. B134, 281 (1984).

[37] F. Boehm et al., Phys. Rev. D 64, 112001 (2001), arXiv:hep-ex/0107009.

[38] B. Armbruster et al. (KARMEN Collaboration), Phys. Rev. D 65, 112001 (2002), arXiv:hepex/0203021.

[39] P. Astier et al. (NOMAD Collaboration), Phys. Lett. B570, 19 (2003), arXiv:hep-ex/0306037

[40] H. Pas, S. Pakvasa, and T. J. Weiler, Phys. Rev. D 72, 095017 (2005), arXiv:hep-ph/0504096

[41] S. Palomares-Ruiz, S. Pascoli, and T. Schwetz, JHEP 09, 048 (2005), arXiv:hep-ph/0505216

[42] A. E. Nelson and J. Walsh, Phys. Rev. D 77, 033001 (2008), arXiv:0711.1363 [hep-ph]. 
[43] A. A. Aguilar-Arevalo et al. (MiniBooNE Collaboration), Phys. Rev. D 79, 072002 (2009), arXiv:0806.1449 [hep-ex] .

[44] A. A. Aguilar-Arevalo et al. (MiniBooNE Collaboration), Phys. Rev. Lett. 103, 061802 (2009), arXiv:0903.2465 [hep-ex].

[45] C. Giunti and M. Laveder, arXiv:1012.0267 [hep-ph].

[46] A. A. Aguilar-Arevalo et al. (SciBooNE Collaboration), arXiv:hep-ex/0601022.

[47] C. H. Llewellyn Smith, Phys. Rept. 3, 261 (1972).

[48] A. A. Aguilar-Arevalo et al. (MiniBooNE Collaboration), Phys. Rev. Lett. 100, 032301 (2008), arXiv:0706.0926 [hep-ex] .

[49] R. A. Smith and E. J. Moniz, Nucl. Phys. B43, 605 (1972).

[50] Q. Wu et al. (NOMAD Collaboration), Phys. Lett. B660, 19 (2008), arXiv:0711.1183 [hep-ex]

[51] P. Adamson et al. (MINOS Collaboration), Phys. Rev. D 81, 072002 (2010), arXiv:0910.2201 [hep-ex].

[52] S. J. Barish et al., Phys. Rev. D 19, 2521 (1979).

[53] N. J. Baker et al., Phys. Rev. D 25, 617 (1982).

[54] S. J. Barish et al., Phys. Rev. D 16, 3103 (1977).

[55] S. Bonetti et al., Nuovo Cim. A38, 260 (1977).

[56] S. Ciampolillo et al. (Gargamelle Neutrino Propane Collaboration), Phys. Lett. B84, 281 (1979).

[57] N. Armenise et al., Nucl. Phys. B152, 365 (1979).

[58] S. V. Belikov et al., Z. Phys. A320, 625 (1985).

[59] G. M. Radecky et al., Phys. Rev. D 25, 1161 (1982).

[60] T. Kitagaki et al., Phys. Rev. D 34, 2554 (1986).

[61] P. S. Auchincloss et al., Z. Phys. C48, 411 (1990).

[62] J. P. Berge et al., Z. Phys. C35, 443 (1987).

[63] V. B. Anikeev et al., Z. Phys. C70, 39 (1996).

[64] A. I. Mukhin et al., Sov. J. Nucl. Phys. 30, 528 (1979).

[65] D. MacFarlane et al., Z. Phys. C26, 1 (1984).

[66] A. A. Aguilar-Arevalo et al. (MiniBooNE Collaboration), Phys. Rev. D 81, 092005 (2010), arXiv:1002.2680 [hep-ex] .

[67] V. Lyubushkin et al. (NOMAD Collaboration), Eur. Phys. J. C63, 355 (2009), arXiv:0812.4543 [hep-ex] .

[68] K. Hiraide et al. (SciBooNE Collaboration), Phys. Rev. D 78, 112004 (2008) 
[69] Y. Kurimoto et al. (SciBooNE Collaboration), Phys. Rev. D 81, 033004 (2010), arXiv:0910.5768 [hep-ex] .

[70] Y. Kurimoto et al. (SciBooNE Collaboration), Phys. Rev. D 81, 111102 (2010), arXiv:1005.0059 [hep-ex] .

[71] Y. Hayato, Nucl. Phys. Proc. Suppl. 112, 171 (2002),

[72] G. Mitsuka (Super-Kamiokande Collaboration), J. Phys. Conf. Ser. 136, 042017 (2008).

[73] D. Casper, Nucl. Phys. Proc. Suppl. 112, 161 (2002), arXiv:hep-ph/0208030.

[74] Y. Fukuda et al. (Kamiokande Collaboration), Phys. Lett. B335, 237 (1994).

[75] K. Nitta et al., Nucl. Instrum. Meth. A535, 147 (2004), arXiv:hep-ex/0406023.

[76] K. Hiraide, Ph.D. thesis, Kyoto University (2009), FERMILAB-THESIS-2009-02.

[77] Y. Kurimoto, Ph.D. thesis, Kyoto University (2010), FERMILAB-THESIS-2010-12.

[78] S. Buontempo et al., Nucl. Instrum. Meth. A349, 70 (1994).

[79] J. A. Crittenden et al., Phys. Rev. D 34, 2584 (1986).

[80] A. A. Aguilar-Arevalo et al. (MiniBooNE Collaboration), Nucl. Instrum. Meth. A599, 28 (2009), arXiv:0806.4201 [hep-ex].

[81] S. J. Brice et al. (MiniBooNE Collaboration), Nucl. Instrum. Meth. A562, 97 (2006), arXiv:1005.3525 [physics.ins-det].

[82] K. B. M. Mahn, Ph.D. thesis, Columbia University (2009), FERMILAB-THESIS-2009-22.

[83] S. Agostinelli et al. (GEANT4 Collaboration), Nucl. Instrum. Meth. A506, 250 (2003).

[84] J. R. Sanford and C. L. Wang, BNL Report No. 11479 (1967), BNL-11479.

[85] M. G. Catanesi et al., Eur. Phys. J. C52, 29 (2007), arXiv:hep-ex/0702024.

[86] I. Chemakin et al. (E910 Collaboration), Phys. Rev. C 77, 015209 (2008), arXiv:0707.2375 [nucl-ex].

[87] N. V. Mokhov et al., arXiv:nucl-th/9812038.

[88] E. J. Moniz et al., Phys. Rev. Lett. 26, 445 (1971).

[89] H. S. Budd, A. Bodek, and J. Arrington, arXiv:hep-ex/0308005.

[90] A. Bodek, S. Avvakumov, R. Bradford, and H. S. Budd, J. Phys. Conf. Ser. 110, 082004 (2008), arXiv:0709.3538 [hep-ex] .

[91] D. Rein and L. M. Sehgal, Ann. Phys. 133, 79 (1981).

[92] D. Rein, Z. Phys. C35, 43 (1987).

[93] D. Rein and L. M. Sehgal, Nucl. Phys. B223, 29 (1983).

[94] D. Rein and L. M. Sehgal, Phys. Lett. B657, 207 (2007), arXiv:hep-ph/0606185.

[95] K. Nakamura et al. (Particle Data Group), J. Phys. G37, 075021 (2010). 
[96] A. A. Aguilar-Arevalo et al. (MiniBooNE Collaboration), Phys. Lett. B664, 41 (2008), arXiv:0803.3423 [hep-ex] .

[97] M. Gluck, E. Reya, and A. Vogt, Eur. Phys. J. C5, 461 (1998), arXiv:hep-ph/9806404.

[98] A. Bodek and U. K. Yang, arXiv:hep-ex/0308007.

[99] M. Nakahata et al. (Kamiokande Collaboration), J. Phys. Soc. Jap. 55, 3786 (1986).

[100] T. Sjostrand, Comput. Phys. Commun. 82, 74 (1994).

[101] D. Ashery et al., Phys. Rev. C 23, 2173 (1981).

[102] R. D. Ransome et al., Phys. Rev. C 45, 509 (1992).

[103] M. K. Jones et al., Phys. Rev. C 48, 2800 (1993).

[104] A. Heikkinen, N. Stepanov, and J. P. Wellisch, in Proceedings of 2003 Conference for Computing in High-Energy and Nuclear Physics (CHEP 03), La Jolla, California, 24-28 Mar 2003 (2003) p. MOMT008, arXiv:nucl-th/0306008.

[105] M. Hasegawa, Ph.D. thesis, Kyoto University (2006).

[106] H. Maesaka, Ph.D. thesis, Kyoto University (2005).

[107] J. L. Alcaraz-Aunion, Ph.D. thesis, Universidad Autonoma de Barcelona (2010).

[108] R. B. Patterson, Ph.D. thesis, Princeton University (2007), FERMILAB-THESIS-2007-19.

[109] G. J. Feldman and R. D. Cousins, Phys. Rev. D 57, 3873 (1998), arXiv:physics/9711021.

[110] I. Stancu et al., arXiv:0910.2698 [hep-ex]].

[111] R. Brun, F. Bruyant, M. Maire, A. C. McPherson, and P. Zanarini, CERN-DD-EE-84-1.

[112] C. Zeitnitz and T. A. Gabriel, Nucl. Instrum. Meth. A349, 106 (1994).

[113] G. Battistoni et al., AIP Conf. Proc. 896, 31 (2007),

[114] R. B. Patterson et al., Nucl. Instrum. Meth. A608, 206 (2009), arXiv:0902.2222 [hep-ex].

[115] A. A. Aguilar-Arevalo, Ph.D. thesis, Columbia University (2008), FERMILAB-THESIS2008-01.

[116] T. Katori, Ph.D. Thesis, Indiana University (2008), FERMILAB-THESIS-2008-64.

[117] D. W. Schmitz, Ph.D. thesis, Columbia University (2008), FERMILAB-THESIS-2008-26.

[118] T. Suzuki, D. F. Measday, and J. P. Roalsvig, Phys. Rev. C 35, 2212 (1987). 